M. Sato and T. Kimura

Nagoya Math. J.

Vol. 65 (1977) 1-155

\title{
A CLASSIFICATION OF IRREDUCIBLE PREHOMOGENEOUS VECTOR SPACES AND THEIR RELATIVE INVARIANTS
}

\author{
M. SATO AND T. KIMURA*
}

\section{Introduction}

Let $G$ be a connected linear algebraic group, and $\rho$ a rational representation of $G$ on a finite-dimensional vector space $V$, all defined over the complex number field $\boldsymbol{C}$.

We call such a triplet $(G, \rho, V)$ a prehomogeneous vector space if $V$ has a Zariski-dense $G$-orbit. The main purpose of this paper is to classify all prehomogeneous vector spaces when $\rho$ is irreducible, and to investigate their relative invariants and the regularity.

This paper consists of the following eight sections.

$\S 1$. Preliminaries

$\S 2$. Castling transforms

$\S 3$. Classification of reduced triplets $(G, \rho, V)$ satisfying $\operatorname{dim} G \geqq \operatorname{dim} V$

$\S 4$. Relative invariants and the regularity

$\S 5$. The prehomogeneity and relative invariants of reduced triplets obtained in $\S 3$

$\S 6$. The semi-simple case

$\S 7$. Table of reduced irreducible prehomogeneous vector spaces

$\S 8$. Prehomogeneous vector spaces with finitely many orbits

We now make a brief survey of this paper. For the convenience of the reader, we shall review, at the beginning of $\S 1$, basic facts about complex simple Lie algebras, especially their irreducible representations and their classification. Then we shall construct a simple Lie algebra of each type and calculate its representation degrees which will be used in $\S 3$. We shall introduce in $\S 2$ an important notion of castling transform, which is an irreducible prehomogeneous vector space obtained from

Received April 16, 1975.

Revised June 23, 1976.

* The second author's research was supported in part by NSF grant MPS72$05055 \mathrm{~A} 03$. 
a given one by a certain process. It will be shown that each prehomogeneous vector space is obtained by successive castling transforms starting from a reduced one, which is characterized by the property that it has the smallest dimension in a "fixed tree" of those transforms.

Our solution of the classification problem consists of the explicit description of the process of castling transform and the table of reduced irreducible prehomogeneous vector spaces which will be given in $\S 7$.

Obviously a triplet $(G, \rho, V)$ is a prehomogeneous vector space only when $\operatorname{dim} G \geq \operatorname{dim} V$, and hence as the first step, we classify in $\S 3$ the reduced triplets satisfying such a condition. To investigate these triplets, we develop in $\S 4$ a general theory about relative invariants and the regularity of prehomogeneous vector spaces. By using the results of $\S 4$, we investigate in $\S 5$ the reduced triplets obtained in $\S 3$, especially we determine their prehomogeneity. By a well-known theorem of E. Cartan, if $\rho$ is irreducible, the Lie algebra $g$ of $\rho(G)$ is reductive with center at most one-dimensional. We have assumed in $\S 3$ and $\S 5$ that the center of $\mathrm{g}$ is one-dimensional. The remaining case will be discussed in $\S 6$. In the last section, we consider an irreducible triplet with finitely many orbits. It will be shown that such a triplet is a reduced irreducible prehomogeneous vector space with few exceptions.

The authors wish to express hearty thanks to Professor T. Shintani for reading the manuscript and making many invaluable suggestions.

\section{§1. Preliminaries}

First of all, we shall review the basic facts about complex simple Lie algebras, especially their irreducible representations and their classification. We denote by $\operatorname{gr}(V)$ the Lie algebra of all linear transformations of a vector space $V$. Similarly we denote by $\mathfrak{g} \mathfrak{h}(n)$ the Lie algebra of all $n \times n$ matrices.

The following two theorems give us a principle to solve the classification problem in the irreducible case.

THEOREM 1 (E. Cartan). Let $d \rho: \mathfrak{g} \rightarrow \mathfrak{g h}(V)$ be an irreducible representation of a Lie algebra $g$ on $V$ over $C$. Then the image $d \rho(\mathfrak{g})$ is reductive with center at most one-dimensional, i.e., a semi-simple Lie

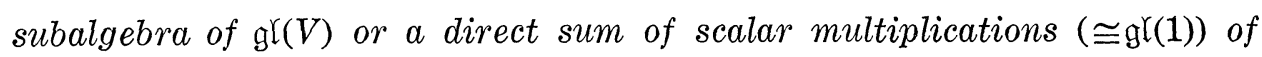
$V$ and a semi-simple Lie subalgebra of gr(V) (see [9]). 
THEOREM 2 (I. Schur). Let $\mathfrak{g}=\mathrm{g}_{1} \oplus \cdots \oplus \mathrm{g}_{\ell}$ be a Lie algebra of a direct sum of Lie algebras $\mathrm{g}_{i}(1 \leq i \leq \ell)$, and $d \rho: \mathfrak{g} \rightarrow \mathfrak{g l}(V)$ an irreducible representation of $\mathfrak{g}$ on the complex vector space $V$. Then there exist irreducible representations $d \rho_{i}: \mathfrak{g}_{i} \rightarrow \mathfrak{g l}\left(V_{i}\right)$ of $\mathrm{g}_{i}$ on $V_{i}(1 \leq i \leq \ell)$ such that (1) $V \cong V_{1} \otimes \cdots \otimes V_{\ell}, \quad$ (2) $d \rho=d \rho_{1} \otimes \cdots \otimes d \rho_{\ell}$, i.e.,

$$
d \rho(X) v=\sum_{i=1}^{\ell} v_{1} \otimes \cdots \otimes d \rho_{i}\left(X_{i}\right) v_{i} \otimes \cdots \otimes v_{\ell}
$$

for $X=\left(X_{1}, \cdots, X_{\ell}\right) \in \mathfrak{g}, v=\left(v_{1} \otimes \cdots \otimes v_{\ell}\right) \in V$ (see [1]).

Definition 3. Let $\mathfrak{g}$ be a semi-simple Lie algebra over $\boldsymbol{C}$. Then there exists a subalgebra $\mathfrak{h}$ of $\mathfrak{g}$ satisfying the two conditions: (1) $\mathfrak{h}$ is a maximal abelian subalgebra, i.e., $[X, Y]=0$ for any $X, Y \in \mathfrak{h}$, and any element $X$ of $g$ satisfying $[X, Y]=0$ for all $Y \in \mathfrak{h}$ belongs to $\mathfrak{h}$. (2) For any $H \in \mathfrak{h}$, the linear endomorphism ad $(H)$ of $\mathfrak{g}$ is diagonalizable (i.e., semi-simple). In this case, $\mathfrak{h}$ is called a Cartan subalgebra. Let $\mathfrak{h}_{1}$ and $\mathfrak{h}_{2}$ be two Cartan subalgebras of $\mathfrak{g}$. Then there exists an automorphism $L$ of $\mathrm{g}$ satisfying $\mathfrak{h}_{1}=L \mathfrak{h}_{2}$. Hence the dimension of a Cartan subalgebra depends only on $g$ which is called the rank of $g$.

DeFinition 4. Let $\mathfrak{g}$ be a semi-simple Lie algebra, $\mathfrak{h}$ its fixed Cartan subalgebra, $d \rho: \mathfrak{g} \rightarrow \operatorname{gr}(V)$ a representation of $\mathfrak{g}$ on $V$. An element $\lambda$ of the dual space $\mathfrak{h}^{*}$ of $\mathfrak{h}$ is called a weight of $d \rho$ if $\mathfrak{g}_{\lambda} \neq\{0\}$, where $\mathfrak{g}_{\lambda}=$ $\{x \in V \mid d \rho(H) x=\lambda(H) x$ for any $H \in \mathfrak{h}\}$. A non-zero element of $\mathrm{g}_{\lambda}$ is called a weight vector. Let $d \rho^{\prime}: \mathfrak{g} \rightarrow \mathfrak{g}\left(V^{\prime}\right)$ be another representation of $g$ on $V^{\prime}$. Then $d \rho$ and $d \rho^{\prime}$ are equivalent if and only if they have same weights. A non-zero weight of the adjoint representation is called a root of $\mathfrak{g}$ (with respect to $\mathfrak{h})$. The totality $\Delta$ of roots is called the root system of $\mathfrak{g}($ w.r.t. $\mathfrak{h})$. If $\alpha \in \Delta$, then $-\alpha \in \Delta, \operatorname{dim} \mathfrak{g}_{\alpha}=1, \mathfrak{g}=\mathfrak{h} \oplus \sum_{\alpha \in \Delta} \mathfrak{g}_{\alpha}$ and hence $\operatorname{dim} g=\operatorname{rank} g+\#(\Delta)$.

DEFINITION 5. Let $\mathfrak{g}$ be any Lie algebra. The symmetric bilinear form $B$ of $g$ defined by $B(X, Y)=\operatorname{Trad} X \circ$ ad $Y$ is called the Killing form of $\mathfrak{g}$. This is non-degenerate if and only if $\mathfrak{g}$ is semi-simple.

Definitiom 6. Let $\mathfrak{g}$ be a semi-simple Lie algebra of rank $\ell$. Then the restriction $\left.B\right|_{\mid}$of the Killing form $B$ to the fixed Cartan subalgebra $\mathfrak{h}$ is also non-degenerate. Hence, for each root $\alpha$, there exists uniquely an element $H_{\alpha}$ of $\mathfrak{h}$ satisfying $\alpha(H)=B\left(H, H_{\alpha}\right)$ for any $H \in \mathfrak{h}$. Let $\mathfrak{h}_{0}$ be 
the vector space over the rational number field $\boldsymbol{Q}$ spanned by $H_{\alpha}$ for all $\alpha \in \Delta$. Then $\operatorname{dim} \mathfrak{h}_{0}=\ell$, and the restriction $\left.B\right|_{\mathfrak{g}_{0}}$ of $B$ to $\mathfrak{h}_{0}$ is a $Q$-valued positive definite symmetric bilinear form. Let $\mathfrak{h}_{0}^{*}$ be the dual vector space of $\mathfrak{h}_{0}$ over $\boldsymbol{Q}$. For each $\lambda \in \mathfrak{h}_{0}^{*}$, there exists uniquely an element $H_{\lambda}$ of $\mathfrak{h}_{0}$ satisfying $\lambda(H)=B\left(H, H_{\lambda}\right)$ for any $H \in \mathfrak{h}_{0}$. Note that $\Delta \subset \mathfrak{h}_{0}^{*}$ and for each $\alpha \in \Delta, H_{\alpha}$ is the same as the previous definition. We can define a positive definite inner product $(\lambda, \mu)$ on $\mathfrak{G}_{0}^{*}$ by $(\lambda, \mu)=B\left(H_{\lambda}, H_{\mu}\right)=\lambda\left(H_{\mu}\right)$ $=\mu\left(H_{2}\right)$.

Definition 7. Fix a basis $H_{1}, \cdots, H_{\ell}$ of $\mathfrak{h}_{0}$ over $\boldsymbol{Q}$. An element $\lambda$ of $\mathfrak{h}_{0}^{*}$ is called positive if $\lambda\left(H_{1}\right)=\cdots=\lambda\left(H_{k-1}\right)=0, \lambda\left(H_{k}\right)>0$ for some $k=1, \cdots, \ell$. We can define a lexicographical order in $\mathfrak{G}_{0}^{*}$. Namely, $\lambda>\mu$ implies that $\lambda-\mu$ is positive for $\lambda, \mu \in \mathfrak{h}_{0}^{*}$.

Definition 8. The totality of positive roots will be denoted by $\Delta_{+}$. A positive root is called simple if it is not a sum of two positive roots. A subset $\Pi=\left\{\alpha_{1}, \cdots, \alpha_{\ell}\right\}$ of $\Delta$ is called a fundamental root system if any root $\alpha$ is written uniquely as $\alpha=m_{1} \alpha_{1}+\cdots+m_{\ell} \alpha_{\ell}$ where all $m_{i}$ are non-negative integers or all $m_{i}$ are non-positive integers. There exist just $\ell$ simple roots $\alpha_{1}, \cdots, \alpha_{\ell}$ and they form a fundamental root system. Conversely, a fundamental root system is the totality of simple roots under some lexicographical order in $\mathfrak{h}_{0}^{*}$.

DEFINITION 9. Let $\mathfrak{n}_{+}$be a vector subspace of $\mathfrak{g}$ generated by $\mathfrak{g}_{\alpha}$ for all $\alpha \in A_{+}$. Let $d \rho: \mathfrak{g} \rightarrow \mathfrak{g} l(V)$ be an irreducible representation of $\mathfrak{g}$ on $V$. Then there exists uniquely, up to constant, a non-zero element $x$ of $V$ such that $d \rho(\nu) x=0$ for any $\nu \in \mathfrak{n}_{+}$. For such $x \in V$, there exists $\Lambda \in \mathfrak{h}^{*}$ such that $d \rho(H) x=\Lambda(H) \cdot x$ for any $H \in \mathfrak{h}$. Moreover, this $\Lambda$ is an element of $\mathfrak{G}_{0}^{*}$ and a dominant integral form, i.e., $\frac{2(\Lambda, \alpha)}{(\alpha, \alpha)}$ is a nonnegative integer for any $\alpha \in \Delta_{+}$. We say that $x$ is a highest weight vector and $\Lambda$ is the highest weight of $d \rho$.

TheOREM 10. Let $\Lambda$ be any dominant integral form of $\mathfrak{h}$. Then there exists an irreducible representation $d \rho: \mathfrak{g} \rightarrow \mathfrak{g l}(V)$ of $\mathfrak{g}$, with the highest weight 4 . This gives a one-to-one correspondence between the equivalence classes of irreducible representations of $\mathrm{g}$ and dominant integral forms. Sometimes we shall denote do by $\Lambda$.

Definition 11. Let $\alpha_{1}, \cdots, \alpha_{\ell}$ be the simple roots w.r.t. $\left(\mathfrak{g}, \mathfrak{h}, \Delta_{+}\right)$. Then there exist dominant integral forms $\Lambda_{1}, \cdots, \Lambda_{\ell}$ uniquely such that 
$\frac{2\left(\Lambda_{i}, \alpha_{j}\right)}{\left(\alpha_{j}, \alpha_{j}\right)}=\delta_{i j}(i, j=1, \cdots, \ell) . \quad$ These $\Lambda_{1}, \cdots, \Lambda_{\ell}$ are called fundamental dominant weights, and the corresponding irreducible representations $d \rho_{i}: \mathfrak{g} \rightarrow \mathfrak{g l}\left(V_{i}\right)(1 \leq i \leq \ell)$ are called the fundamental irreducible representations of $\mathrm{g}$. Any dominant integral form $\Lambda$ is of the form $\Lambda=$ $\sum_{i=1}^{\ell} m_{i} \Lambda_{i}$ where each $m_{i}$ is a non-negative integer. Let $v_{i}$ be the highest weight vector of $d \rho_{i}(1 \leq i \leq \ell)$, and let $V$ be the least $g$-invariant subspace of $V_{1} \overbrace{\otimes \cdots \otimes}^{m_{1}} V_{1} \otimes \cdots \otimes V_{\ell} \overbrace{\otimes \cdots \otimes}^{m_{\ell}} V_{\ell}$ containing $v=v_{1} \overbrace{\otimes \cdots \otimes}^{m_{1}}$ $v_{1} \otimes \cdots \otimes v_{\ell} \overbrace{\otimes \cdots \otimes}^{m_{\ell}} v_{\ell}$. Then the restriction $d \rho$ of $d \rho_{1} \otimes \overbrace{\cdots \otimes}^{m_{1}} d \rho_{1} \otimes \cdots \otimes$ $d \rho_{\ell} \overbrace{\otimes \cdots \otimes}^{m_{\ell}} d \rho_{\ell}$ to $V$ is an irreducible representation of $\mathrm{g}$ with the highest weight $\Lambda=\sum m_{i} \Lambda_{i}$. In view of Theorem 10, the dimension of $V$ depends only on the corresponding dominant integral form $\Lambda$, and hence we denote it by $d(\Lambda)$ which is called a representation degree of $\Lambda$ (or $d \rho$ ).

THEOREM 12 (Weyl's dimension formula).

$$
d(\Lambda)=\prod_{\alpha \in \Delta_{+}} \frac{(\Lambda+\rho, \alpha)}{(\rho, \alpha)} \text { where } \rho=\frac{1}{2} \sum_{\alpha \in \Delta_{+}} \alpha .
$$

COROLlaRY 13. Let $\Lambda=\sum_{i=1}^{\ell} m_{i} \Lambda_{i}$ and $\Lambda^{\prime}=\sum_{i=1}^{\ell} m_{i}^{\prime} \Lambda_{i}$ be dominant integral forms such that $m_{i} \geqq m_{i}^{\prime}$ for each $i=1, \cdots, l$ and $\Lambda \neq \Lambda^{\prime}$. Then $d(\Lambda)>d\left(\Lambda^{\prime}\right)$.

Proof. For any positive root $\alpha=\sum_{i=1}^{\ell} n_{i} \alpha_{i} \quad\left(n_{i} \geqq 0\right), \quad(\Lambda, \alpha)=$ $\sum_{i, j} m_{i} n_{j}\left(\Lambda_{i}, \alpha_{j}\right)=\frac{1}{2} \sum_{i} m_{i} n_{i}\left(\alpha_{i}, \alpha_{i}\right)>\frac{1}{2} \sum_{i} m_{i}^{\prime} n_{i}\left(\alpha_{i}, \alpha_{i}\right)=\left(\Lambda^{\prime}, \alpha\right)$ and this implies $d(\Lambda)>d\left(\Lambda^{\prime}\right)$.

Q.E.D.

Now, we shall review the classification of simple Lie algebras over $\boldsymbol{C}$.

DEFINITION 14. Let $R^{\ell}$ be an $\ell$-dimensional vector space over the real number field $\boldsymbol{R}$ with a positive definite inner product (, ). Define the length $\|\alpha\|$ of $\alpha \in R^{\ell}$ as $\|\alpha\|=\sqrt{(\alpha, \alpha)}$ and denote the angle of two vectors $\alpha_{i}, \alpha_{j} \in R^{\ell}$ by $\widehat{\alpha_{i} \alpha_{j}}$.

A subset $\Pi=\left\{\alpha_{1}, \cdots, \alpha_{\ell}\right\}$ of $R^{\ell}$ is called an irreducible admissible system if the following three conditions are satisfied: (1) $\alpha_{1}, \cdots, \alpha_{\ell}$ are linearly independent.

(2) $-\frac{2\left(\alpha_{i}, \alpha_{j}\right)}{\left(\alpha_{j}, \alpha_{j}\right)}$ is a non-negative integer if $i \neq j$.

(3) There is no decomposition of $\Pi$ such that $\Pi=\Pi_{1} \cup \Pi_{2}$ and $\Pi_{1} \perp \Pi_{2}$, 
where $\Pi_{1} \perp \Pi_{2}$ implies that $(\alpha, \beta)=0$ for any $\alpha \in \Pi_{1}$ and $\beta \in \Pi_{2}$.

LEMMA 15. Let $I I=\left\{\alpha_{1}, \cdots, \alpha_{\ell}\right\}$ be an irreducible admissible system of $R^{\ell}$. If $\alpha_{i}, \alpha_{j} \in \Pi, \alpha_{i} \neq \alpha_{j}$ and $\left\|\alpha_{i}\right\| \geqq\left\|\alpha_{j}\right\|$, then they satisfy one of the following conditions.

(1) $\widehat{\alpha_{i} \alpha_{j}}=\frac{\pi}{2}$ i.e. $\left(\alpha_{i}, \alpha_{j}\right)=0$

(2) $\widehat{\alpha_{i} \alpha_{j}}=\frac{2}{3} \pi$ and $\left\|\alpha_{i}\right\|=\left\|\alpha_{j}\right\|$

(3) $\widehat{\alpha_{i} \alpha_{j}}=\frac{3}{4} \pi$ and $\left\|\alpha_{i}\right\|=\sqrt{2}\left\|\alpha_{j}\right\|$

(4) $\widehat{\alpha_{i} \alpha_{j}}=\frac{5}{6} \pi$ and $\left\|\alpha_{i}\right\|=\sqrt{3}\left\|\alpha_{j}\right\|$

DEFINITION 16. Let $\Pi=\left\{\alpha_{1}, \cdots, \alpha_{\ell}\right\}$ be an irreducible admissible system in $R^{\ell}$. To each vector $\alpha_{i} \in \Delta$, associate a vertex and connect the two vertices associated to $\alpha_{i}$ and $\alpha_{j}$ if and only if $\left(\alpha_{i}, \alpha_{j}\right) \neq 0$, i.e., $\widehat{\alpha_{i} \alpha_{j}} \neq \frac{\pi}{2}$. We connect vertices corresponding to $\alpha_{i}$ and $\alpha_{j}$ with a single, double, or triple line according to whether $\widehat{\alpha_{i} \alpha_{j}}=\frac{2}{3} \pi, \frac{3}{4} \pi, \frac{5}{6} \pi$ respectively (see Lemma 15). The arrows point from a longer to a shorter vector, when the lengths are different. Thus we obtain a connected diagram which is called the Dynkin diagram of $I I$.

Lemma 17. Let $\Pi$ be an irreducible admissible system of $R^{\ell}$. Then its Dynkin diagram is one of the following diagrams.

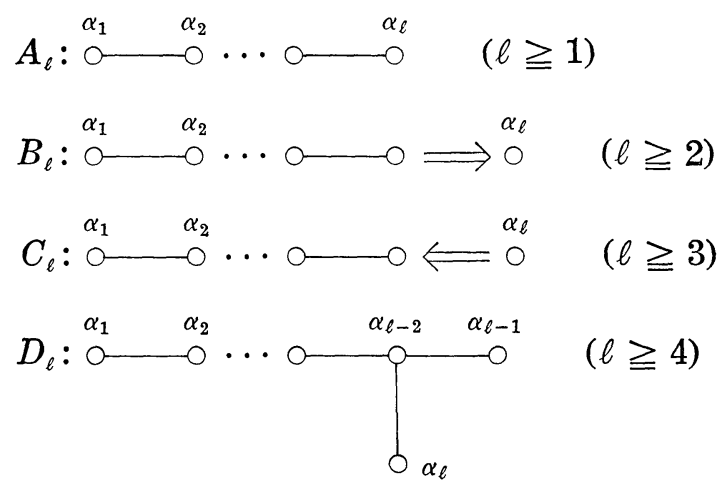




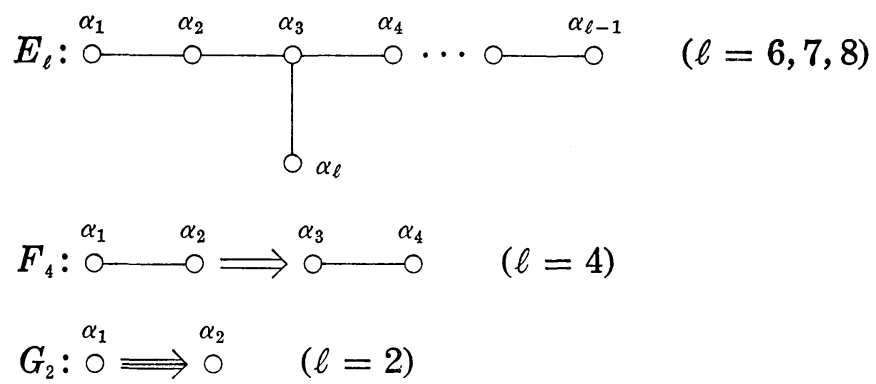

DEFinition 18. Let $g$ be a semi-simple Lie algebra of rank $\ell$ over $\boldsymbol{C}$, and let $\mathfrak{h}$ be a fixed Cartan subalgebra. We can extend the inner product of $\mathfrak{h}_{0}^{*}$ (see Definition 6) to a positive definite inner product of $\ell$-dimensional vector space $R^{\ell}=\mathfrak{H}_{0}^{*} \otimes_{Q} \boldsymbol{R}$ over $\boldsymbol{R}$. Let $\Pi=\left\{\alpha_{1}, \cdots, \alpha_{\ell}\right\}$ be a fundamental root system of $g$. Then, $g$ is simple if and only if $\Pi$ satisfies the third condition in Definition 14, i.e., there is no decomposition such that $\Pi=\Pi_{1} \cup \Pi_{2}$ and $\Pi_{1} \perp \Pi_{2}$. Moreover, if $\mathrm{g}$ is a simple Lie algebra, $\Pi$ is an irreducible admissible system of $R^{\ell}$, and we can get its Dynkin diagram by Definition 16. This diagram depends only on $\mathfrak{g}$, and we can call it the Dynkin diagram of a simple Lie algebra $\mathrm{g}$.

Theorem 19 (Classification of simple Lie algebras). Two simple Lie algebras $\mathfrak{g}_{1}$ and $\mathrm{g}_{2}$ are isomorphic over $C$ if and only if they have the same Dynkin diagrams. Thus Lemma 17 says that a simple Lie algebra over $C$ is isomorphic to one of $A_{\ell}(\ell \geqq 1), B_{\ell}(\ell \geqq 2) C_{\ell}(\ell \geqq 3), D_{\ell}(\ell \geqq 4)$, $E_{\ell}(\ell=6,7,8), F_{4}, G_{2}$.

Definition 20. The simple Lie algebras of type $A_{\ell}, B_{\ell}, C_{\ell}, D_{\ell}$ are called classical Lie algebras, and those of type $E_{\ell}, F_{4}, G_{2}$ are called exceptional Lie algebras.

We shall construct the all types of simple Lie algebras in Theorem 19 and calculate their representation degrees which will be used in $\S 3$.

EXAmPLe 21. Let $\mathfrak{g}=\not{g}(n, C)$ be a subalgebra $\{X \in \mathfrak{g} \mathfrak{r}(n, C) \mid \operatorname{Tr} X=0\}$ of $\mathfrak{g l}(n, C)$. Then the Killing form $B$ of $\mathfrak{g}$ is given by $B(X, Y)=$ $\operatorname{Trad} X \circ$ ad $Y=2 n \operatorname{Tr} X Y$ for any $X, Y \in \mathrm{g}$. Since this is non-degenerate, $\mathrm{g}$ is a semi-simple Lie algebra. We may take as a Cartan subalgebra $\mathfrak{h}$ the totality of diagonal matrices of trace zero. Let $E_{i j}$ be a matrix unit with $(i, j)$-element 1 , all remaining entries zero. Denote an element $\lambda$ of $\mathfrak{h}^{*}$ by $\sum_{i=1}^{n} a_{i} \lambda_{i}\left(a_{i} \in \boldsymbol{C}\right)$ if $\lambda(H)=\sum_{i=1}^{n} a_{i} \lambda_{i}$ for $H=\sum_{i=1}^{n} \lambda_{i} E_{i i} \in \mathfrak{h}$. Then 
the root system $\Delta$ of $\mathfrak{g}$ w.r.t.h is given by $\Delta=\left\{\lambda_{i}-\lambda_{j} \mid i \neq j, i, j=1\right.$, $\cdots, n\}$ and $\mathfrak{g}_{i_{i}-\lambda_{j}}=C \cdot E_{i j}$. We have $\operatorname{dim} \mathfrak{g}=n^{2}-1$, rank $\mathfrak{g}=n-1$. Put $\alpha_{i}=\lambda_{i}-\lambda_{i+1}$ for $i=1, \cdots, n-1$. Since $\lambda_{i}-\lambda_{j}=\alpha_{i}+\alpha_{i+1}+\cdots+\alpha_{j-1}$ $(i<j)$ and $\lambda_{i}-\lambda_{j}=-\left(\alpha_{j}+\alpha_{j+1}+\cdots+\alpha_{i-1}\right)(i>j), \Pi=\left\{\alpha_{1}, \cdots, \alpha_{n-1}\right\}$ is a fundamental root system. For each root $\lambda_{i}-\lambda_{j}$, we shall calculate $H_{\lambda_{i}-\lambda_{j}}$. Put $H_{\lambda_{i}-\lambda_{j}}=\sum_{k=1}^{n} \mu_{k} E_{k k}$. Then, by the definition, $B\left(H, H_{i_{i}-\lambda_{j}}\right)=2 n \sum \lambda_{k} \mu_{k}=$ $\lambda_{i}-\lambda_{j}$ for any $H=\sum_{k=1}^{n} \lambda_{k} E_{k k} \in \mathfrak{h}$. Thus we get that $\mu_{i}=\frac{1}{2 n}, u_{j}=-\frac{1}{2 n}$, and $\mu_{k}=0$ for $k \neq i, j$, i.e., $H_{\lambda_{i}-\lambda_{j}}=\frac{1}{2 n}\left(E_{i i}-E_{j j}\right)$. The fundamental root system $\Pi=\left\{\alpha_{1}, \cdots, \alpha_{n-1}\right\}$ is the totality of simple roots under the lexicographical order in $\mathfrak{h}_{0}^{*}$ defined by a basis $H_{\lambda_{1}-\lambda_{n}}, \cdots, H_{\lambda_{n-1}-\lambda_{n}}$ of $\mathfrak{h}_{0}$. Moreover, by the definition,

$$
\left(\alpha_{i}, \alpha_{j}\right)=B\left(H_{\alpha_{i}}, H_{\alpha_{j}}\right)=\left\{\begin{array}{cl}
0 & |i-j| \geqq 2 \\
-\frac{1}{2 n} & |i-j|=1 \\
\frac{1}{n} & i=j
\end{array}\right.
$$

and this shows that $\Pi$ is an irreducible admissible system and its Dynkin diagram is of type $A_{n-1}$. Thus, $\mathrm{g}=\operatorname{gl}(n, C)$ is a simple Lie algebra of type $A_{n-1}: A_{n-1}=\mathfrak{g l}(n, C)$.

We shall determine the fundamental dominant weights $\Lambda_{i}=\sum_{k=1}^{n} m_{i k} \lambda_{k}$ for $i=1, \cdots, n-1$. We may assume that $m_{i n}=0$ because $\sum_{i=1}^{n} \lambda_{i}=0$. Since $\frac{2\left(\Lambda_{i}, \alpha_{j}\right)}{\left(\alpha_{\alpha}, \alpha_{j}\right)}=m_{i, j}-m_{i, j+1}=\delta_{i j}$ for $i, j=1, \cdots, n-1$, and $m_{i n}=0$, we get that $m_{i j}=1$ for $j \leq i$ and $m_{i j}=0$ for $j>i$, i.e., $\Lambda_{i}=\lambda_{1}+\lambda_{2}+$ $\cdots+\lambda_{i}$ for $i=1, \cdots, n-1$.

As $\Delta_{+}=\left\{\lambda_{i}-\lambda_{j} \mid i<j\right\}, \mathfrak{n}_{+}=\sum_{\alpha \in \Delta_{+}} g_{\alpha}=\sum_{i<j} C E_{i j}$ is the totality of upper triangular matrices with diagonal elements 0 (see Definition 9). Let $V_{1}$ be a $n$-dimensional vector space over $C$ spanned by $u_{1}, \cdots, u_{n}$. Define a representation $d \rho_{1}$ of $\mathfrak{g}$ by $\left(u_{1}, \cdots, u_{n}\right) \mapsto\left(u_{1}, \cdots, u_{n}\right) A$ for any $A \in \mathfrak{g}=\mathfrak{g l}(n, C)$. Then $d \rho_{1}$ is the fundamental irreducible representation of $g$ with the highest weight $\Lambda_{1}$ and $u_{1}$ is the highest weight vector since $d \rho_{1}(\nu) u_{1}=0$ for any $\nu \in \mathfrak{n}_{+}$and $d \rho_{1}(H) u_{1}=\lambda_{1} H$ for any $H=\sum \lambda_{i} E_{i i} \in \mathfrak{h}$. In general, let $V_{k}(1 \leq k \leq n-1)$ be a $\left(\begin{array}{l}n \\ k\end{array}\right)$-dimensional vector space over $\boldsymbol{C}$ spanned by exterior tensor products $u_{i_{1}} \wedge \cdots \wedge u_{i_{k}}\left(1 \leq i_{1}<\ldots<i_{k} \leq n\right)$. 
Define a representation $d \rho_{k}$ of $\mathrm{g}$ by $d \rho_{k}(A)\left(u_{i_{1}} \wedge \cdots \wedge u_{i_{k}}\right)=\sum_{j=1}^{k} u_{i_{1}} \wedge$ $\cdots \wedge d \rho_{1}(A) u_{i_{j}} \wedge \cdots \wedge u_{i_{k}}$. Then $d \rho_{k}$ is the fundamental irreducible representation of $g$ with the highest weight $\Lambda_{k}=\lambda_{1}+\cdots+\lambda_{k}$ since $d \rho_{k}(\nu)\left(u_{1} \wedge \cdots \wedge u_{k}\right)=0$ for any $\nu \in \mathfrak{H}_{+}$and $d \rho(H) u_{1} \wedge \cdots \wedge u_{k}=\left(\lambda_{1}+\right.$ $\left.\cdots+\lambda_{k}\right) u_{1} \wedge \cdots \wedge u_{k}$ for any $H=\sum_{i=1}^{n} \lambda_{i} E_{i i} \in \mathfrak{h}$. In particular, we obtain that $d\left(\Lambda_{k}\right)=\left(\begin{array}{l}n \\ k\end{array}\right)$ for $1 \leq k \leq n$. In view of Definition 11, any irreducible representation space of $\mathfrak{g}(n, C)$ is obtained from a tensor product of $V_{1}$.

Although one can use the Weyl's dimension formula to calculate $d(\Lambda)=\operatorname{dim} V$, there is a simple method for $\mathfrak{g}=\mathfrak{g l}(n, C)$ which is also obtained from the Weyl's dimension formula. We shall introduce this method.

DeFinition 22. To a dominant integral weight $\Lambda=m_{k} \Lambda_{k}(1 \leq k \leq$ $n-1)$, we shall attach the diagram

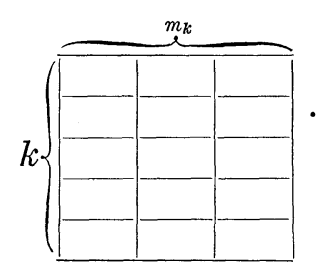

In general, to $\Lambda=m_{1} \Lambda_{1}+\cdots+m_{n-1} \Lambda_{n-1}$ we shall attach the diagram

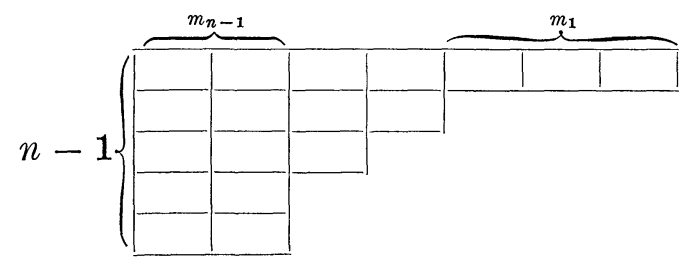

which is called the Young diagram (in detail, see [1]).

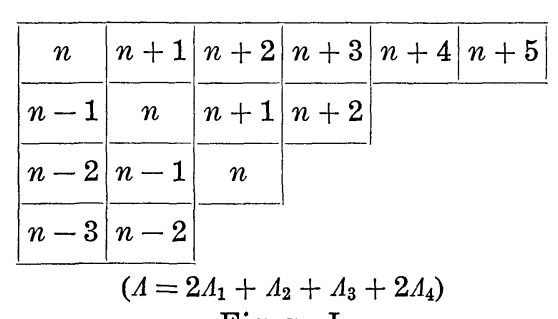

Figure I. 
Write down the number $n$ in each diagonal and to the right direction the increasing numbers, and to the down direction the decreasing numbers one by one like in Figure 1. Then, multiply all of them, which we shall denote by $d_{0}(\Lambda)$.

\begin{tabular}{|l|l|l|l|l|l|}
\hline 9 & 8 & 6 & 4 & 2 & 1 \\
\hline 6 & 5 & 3 & 1 & & \\
\hline 4 & 3 & 1 & & \\
& &
\end{tabular}

Figure II.

Write down the number of the hook's length, i.e., the number of squares of right and down side including itself, like in Figure II. Then multiply all of them which we shall denote by $d_{\infty}(\Lambda)$.

THEOREM 23. Let $\Lambda$ be a dominant integral form of $\mathfrak{g}=\mathfrak{Z l}(n, C)$. Then the dimension $d(\Lambda)$ is given by

$$
d(\Lambda)=\frac{d_{0}(\Lambda)}{d_{\infty}(\Lambda)}
$$

EXAMPLE 24. (1) Let $d \rho$ be an irreducible representation of $\mathfrak{g}=$ $\mathcal{E l}(n, C)$ with the highest weight $\Lambda=\sum_{i=1}^{n-1} m_{i} \Lambda_{i}$. Then the highest weight $\Lambda^{\prime}$ of the contragredient representation $d \rho^{*}$ of $d \rho$ is given by $\Lambda^{\prime}=\sum_{i=1}^{n-1} m_{n-i} \Lambda_{i}$. Thus we obtain that $d\left(\sum_{i=1}^{n-1} m_{i} \Lambda_{i}\right)=d\left(\sum_{i=1}^{n-1} m_{n-i} \Lambda_{i}\right)$.

(2) $\quad d\left(\Lambda_{1}+\Lambda_{\nu}\right)=\frac{(n+1) ! \nu}{(n-\nu) !(\nu+1) !} \quad(1 \leq \nu \leq n-1)$

\begin{tabular}{|c|c|c|}
\hline$n$ & $n+1$ & $|\nu+1|$ \\
\hline$n-1$ & & $\nu-1$ \\
\hline : & & : \\
\hline$n-\nu+1$ & & 1 \\
\hline
\end{tabular}

Figure III.

From the Young diagram in Figure III and Definition 22, we obtain that $d_{0}(\Lambda)=(n+1) n(n-1) \cdots(n-\nu+1)=\frac{(n+1) !}{(n-\nu) !}$ and $d_{\infty}(\Lambda)=$ $(\nu-1) !(\nu+1)=\frac{(\nu+1) !}{\nu}$. Hence by Theorem 23, we obtain our result. 
In particular,

(i ) $d\left(2 \Lambda_{1}\right)=\frac{1}{2} n(n+1)$,

(ii) $d\left(\Lambda_{1}+\Lambda_{2}\right)=\frac{1}{3} n\left(n^{2}-1\right)$,

(iii) $d\left(\Lambda_{1}+\Lambda_{n-2}\right)=\frac{1}{2} n(n+1)(n-2)$,

(iv) $d\left(\Lambda_{1}+\Lambda_{n-1}\right)=n^{2}-1$

(v) $d\left(\Lambda_{3}+\Lambda_{1}\right)=\frac{1}{8} n(n-2)\left(n^{2}-1\right)$

(3) $\quad d\left(\Lambda_{2}+\Lambda_{\nu}\right)=\frac{(n+1) ! n(\nu-1)}{(n-\nu) !(\nu+1) ! 2} \quad(2 \leq \nu \leq n-1)$

\begin{tabular}{|c|c|c|c|}
\hline$n$ & $n+1$ & $\nu+1$ & 2 \\
\hline$n-1$ & $n$ & $\frac{\nu}{1}$ \\
\hline$n-2$ & & $\frac{\nu-2}{}$ \\
\hline$n-\nu+1$ & & \\
\hline$n-1$ & \\
\hline
\end{tabular}

Figure IV.

From the Young diagram in Figure IV and Definition 22, we obtain that $d_{0}(\Lambda)=(n+1) n^{2}(n-1) \cdots(n-\nu+1)=\frac{(n+1) ! n}{(n-\nu) !}$ and $d_{\infty}(\Lambda)=2(\nu+$ 1) $\nu(\nu-2) !=\frac{2(\nu+1) !}{(\nu-1)}$. Hence by Theorem 23 we obtain our result. In particular,

(i ) $d\left(2 \Lambda_{2}\right)=\frac{1}{12} n^{2}\left(n^{2}-1\right)$

(ii) $d\left(\Lambda_{2}+\Lambda_{n-2}\right)=\frac{1}{4} n^{2}(n+1)(n-3)$

(iii) $d\left(\Lambda_{3}+\Lambda_{2}\right)=\frac{1}{24} n^{2}\left(n^{2}-1\right)(n-2)$

(4) $\quad d\left(\Lambda_{3}+\Lambda_{\nu}\right)=\frac{(n+1) ! n(n-1)(\nu-2)}{(n-\nu) !(\nu+1) ! 6} \quad(3 \leq \nu \leq n-1)$

\begin{tabular}{|c|c|}
\hline$n$ & $n+1$ \\
\hline$n-1$ & $n$ \\
\hline$n-2$ & $n-1$ \\
\hline$n-3$ & \\
\hline$:$ & \\
\hline$n-\nu+1$ & \\
\hline
\end{tabular}

\begin{tabular}{|c|c|}
\hline$\nu+1$ & 3 \\
\hline$\nu$ & 2 \\
\hline$\nu-1$ & 1 \\
\hline$\nu-3$ & \\
\hline$:$ & \\
\hline 1 & \\
\hline
\end{tabular}

Figure V.

From the Young diagram in Figure V and Definition 22, we obtain that $d_{0}(\Lambda)=(n+1) n^{2}(n-1)^{2}(n-2) \cdots(n-\nu+1)=\frac{(n+1) ! n(n-1)}{(n-\nu) !}$ and 
$d_{\infty}(\Lambda)=3 \cdot 2 \cdot(\nu+1) \nu(\nu-1)(\nu-3) !=\frac{(\nu+1) ! 6}{(\nu-2)}$. Hence by Theorem 23 we obtain our result. In particular,

(i ) $d\left(2 \Lambda_{3}\right)=\frac{1}{144}(n+1) n^{2}(n-1)^{2}(n-2)$

(ii) $d\left(\Lambda_{3}+\Lambda_{n-1}\right)=\frac{1}{6} n\left(n^{2}-1\right)(n-3)$

(iii) $d\left(\Lambda_{3}+\Lambda_{\nu+1}\right) / d\left(\Lambda_{3}+\Lambda_{\nu}\right)=\frac{(\nu-1)(n-\nu)}{\left(\nu^{2}-4\right)} \quad(\nu \geqq 3)$

(5) $d\left(2 \Lambda_{1}+\Lambda_{n-1}\right)=\frac{1}{2} n(n-1)(n+2)$
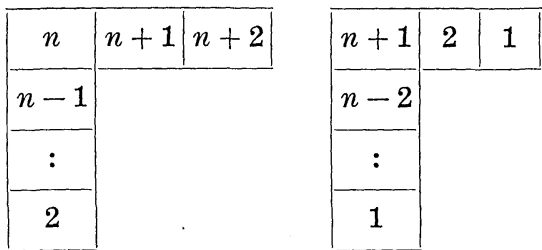

Figure VI.

From the Young diagram in Figure VI and Definition 22, we obtain that $d_{0}(\Lambda)=(n+2)$ ! and $d_{\infty}(\Lambda)=2(n+1) \cdot(n-2)$ !. Thus we obtain our result by Theorem 23 .

(6) $d\left(m \Lambda_{1}\right)=\frac{n(n+1) \cdots(n+m-1)}{m !}=\frac{(n+m-1) !}{m !(n-1) !}$

\begin{tabular}{|c|c|c|c|}
\hline$n$ & $n+1$ & $\cdots$ & $n+m-1$ \\
\hline
\end{tabular}

Figure VII.

From the Young diagram in Figure VII and Theorem 23, we obtain our result. We shall construct the representation $d \rho$ with the highest weight $m \Lambda_{1}$. Let $V_{1}$ be a $n$-dimensional vector space over $C$ spanned by $u_{1}, \cdots, u_{n}$. Define a representation $d \rho_{1}$ of $\mathrm{g}$ by $\left(u_{1}, \cdots, u_{n}\right) \mapsto\left(u_{1}, \cdots, u_{n}\right) A$ for any $A \in \mathfrak{g}=\mathfrak{g l}(n, C)$. Then as we saw in Example 21, this is a representation with the highest weight $\Lambda_{1}$. Let $V$ be a $\frac{(n+m-1) !}{m !(n-1) !}$-dimensional vector space of complex homogeneous polynomials of degree $m$ in variables $u_{1}, \cdots, u_{n}$. Define a representation $d \rho$ on $V$ by $d \rho(A)\left(u_{i_{1}} \cdots u_{i_{m}}\right)$ $=\sum_{j=1}^{m} u_{i_{1}} \cdots\left(d \rho_{1}(A) u_{i_{j}}\right) \cdots u_{i_{m}}$. Then $d \rho$ is an irreducible representation with the highest weight $m \Lambda_{1}$ and the highest weight vector $u_{i}^{m}$. 
(7) If $m=2$, i.e., $\Lambda=2 \Lambda_{1}(=\square)$, we can construct a representation $d \rho$ in another way. Namely, let $V$ be the totality of $n \times n$ symmetric matrices and define $d \rho$ by $d \rho(A) X=A X+X^{t} A$ for any $A \in \mathfrak{g}=$ $\mathfrak{g l}(n, C), X \in V$. Then $d \rho(\nu) E_{11}=0$ for any $\nu \in \mathfrak{n}_{+}$and $d \rho(H) E_{11}=2 \lambda_{1} E_{11}$ for any $H=\sum_{i=1}^{n} \lambda_{i} E_{i i} \in \mathfrak{h}$. Thus $d \rho$ is an irreducible representation of $\mathfrak{I l}(n, C)$ with the highest weight $2 \Lambda_{1}$. If we take $V$ as the totality of $n \times n$ skew-symmetric matrices and define $d \rho$ by $d \rho(A) X=A X+X^{t} A$ for any $A \in \mathfrak{g}=\mathfrak{g l}(n, C), X \in V$, then $d \rho$ is an irreducible representation with the highest weight $\Lambda_{2}=\lambda_{1}+\lambda_{2}(=-)$.

ExAmple 25. Let $\mathfrak{s p}(n, C)=\left\{\left.A \in \mathfrak{g} \mathfrak{\mathfrak { l } ( 2 n , C )}\right|^{t} A J+J A=0\right\}$ be a Lie subalgebra of $\mathfrak{g l}(2 n, C)$ where $J=\left(\frac{0}{-I_{n}} \mid \frac{I_{n}}{0}\right)$. Then by the definition, an element $A$ of $\mathfrak{g} \mathfrak{l}(2 n, C)$ is in $\mathfrak{g}=\mathfrak{g h}_{\mathfrak{p}}(n, C)$ if and only if $A$ is of the form:

$$
A=\left(\frac{X}{Z} \mid \frac{Y}{{ }^{-t} X}\right), \quad{ }^{t} Y=Y,{ }^{t} Z=Z, X, Y, Z \in M(n, C) .
$$

In particular, we have $\operatorname{dim} \mathfrak{s p}(n, C)=n(2 n+1)$. The Killing form $B$ of $\mathfrak{g}=\mathfrak{s p}(n, C)$ is given by $B(U, V)=\operatorname{Trad} U$ ad $V=(2 n+2) \operatorname{Tr} U V$ for any $U, V \in \mathfrak{g}$ and as this is non-degenerate, $\mathfrak{g}=\mathfrak{g p}(n, C)$ is semi-simple. Define elements $H\left(\lambda_{1}, \cdots, \lambda_{n}\right), E_{ \pm \lambda_{i} \pm \lambda_{j}}$ of $g$ as follows.

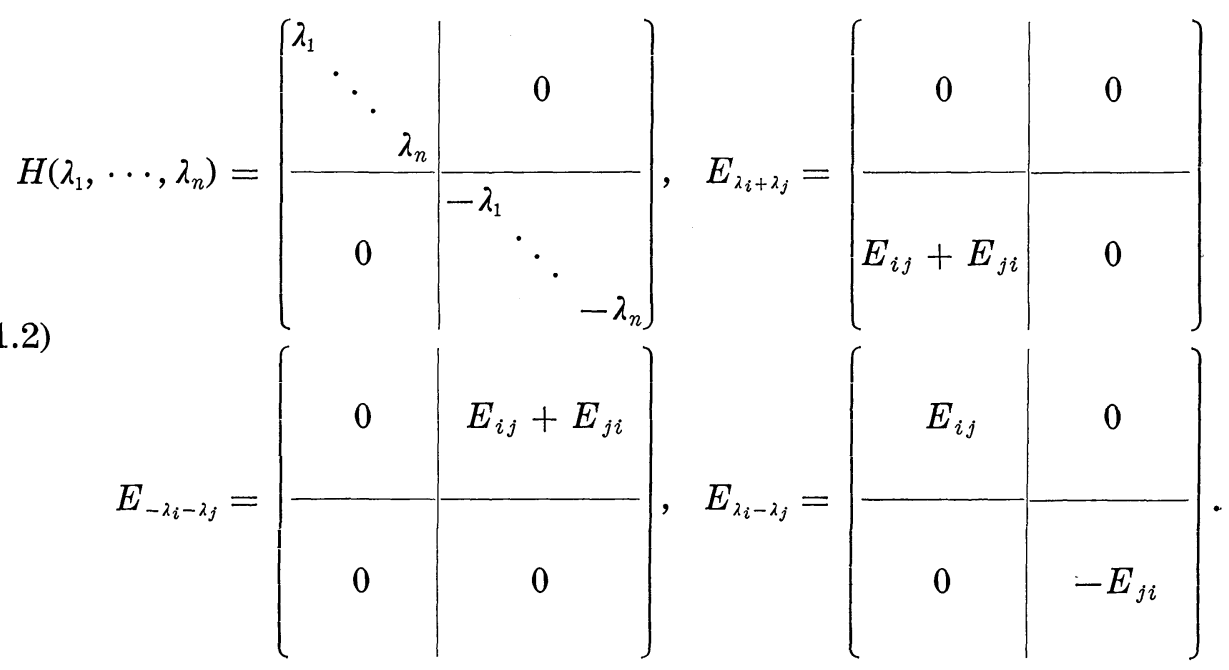

Then the totality $\mathfrak{h}$ of $H\left(\lambda_{1}, \cdots, \lambda_{n}\right)$ is a Cartan subalgebra of $\mathfrak{g}=\mathfrak{s} \mathfrak{p}(n, C)$ and as $\operatorname{ad}(H) E_{ \pm \lambda_{i} \pm \lambda_{j}}=\left( \pm \lambda_{i} \pm \lambda_{j}\right) E_{ \pm \lambda_{i} \pm \lambda_{j}}, \Delta=\left\{ \pm \lambda_{i} \pm \lambda_{j} \mid i, j=1, \cdots, n\right\}$ is the 
root system of $\mathrm{g}$ w.r.t.h. Put $\alpha_{1}=\lambda_{1}-\lambda_{2}, \alpha_{2}=\lambda_{2}-\lambda_{3}, \cdots, \alpha_{n-1}=\lambda_{n-1}$ $-\lambda_{n}, \alpha_{n}=2 \lambda_{n}$. Then $\Pi=\left\{\alpha_{1}, \cdots, \alpha_{n}\right\}$ is a fundamental root system because $\lambda_{i}-\lambda_{j}=\alpha_{i}+\alpha_{i+1}+\cdots+\alpha_{j-1}$ for $i<j, \lambda_{i}-\lambda_{j}=-\left(\alpha_{j}+\alpha_{j+1}+\cdots+\alpha_{i-1}\right)$ for $i>j$, and $\pm\left(\lambda_{i}+\lambda_{j}\right)= \pm\left(\left(\lambda_{i}-\lambda_{n}\right)+\left(\lambda_{j}-\lambda_{n}\right)+\alpha_{n}\right)$. As

$$
H_{\alpha_{i}}=\frac{1}{4(n+1)} H(0, \ldots, \stackrel{i}{1}, \stackrel{i+1}{1}, \ldots, 0)
$$

for $1 \leq i \leq n-1$ and $H_{\alpha_{n}}=\frac{1}{4(n+1)} H(0, \cdots, 0,2)$, we obtain that $\left(\alpha_{i}, \alpha_{j}\right)=0$ for $|i-j| \geqq 2,\left(\alpha_{i}, \alpha_{j}\right)=-\frac{1}{4(n+1)}$ for $|i-j|=1$ and $1 \leq i$, $j \leq n-1,\left(\alpha_{n-1}, \alpha_{n}\right)=-\frac{1}{2(n+1)},\left(\alpha_{i}, \alpha_{i}\right)=\frac{1}{2(n+1)}$ for $i=1, \cdots, n-1$, and $\left(\alpha_{n}, \alpha_{n}\right)=\frac{1}{n+1}$. This shows that $\mathfrak{g}=\mathfrak{g h}(n, C)$ is a simple Lie algebra of type $C_{n}$. The fundamental dominant weights are $\Lambda_{1}=\lambda_{1}, \Lambda_{2}=$ $\lambda_{1}+\lambda_{2}, \cdots, \Lambda_{n}=\lambda_{1}+\lambda_{2}+\cdots+\lambda_{n}$.

Let $V_{1}$ be a $2 n$-dimensional vector space over $C$ spanned by $u_{1}$, $\cdots, u_{2 n}$. For $1 \leq k \leq n$, put $T^{k}\left(V_{1}\right)=V_{1} \overbrace{\otimes \cdots \otimes}^{k} V_{1}$ and define a map $\varphi: T^{k}\left(V_{1}\right) \rightarrow T^{k-2}\left(V_{1}\right)$ by $\varphi\left(u_{1} \otimes \cdots \otimes u_{k}\right)=\varepsilon\left(u_{1}, u_{2}\right) u_{3} \otimes \cdots \otimes u_{k}$ where $\varepsilon$ is a skew-symmetric bilinear form on $V_{1}$ such that $\varepsilon\left(u_{i}, u_{i+n}\right)=1(1 \leq i \leq n)$ and $\varepsilon\left(u_{i}, u_{j}\right)=0(i<j$ and $j \neq i+n)$. Let $V_{k}=\Lambda^{k}\left(V_{1}\right)$ be a vector space over $C$ spanned by exterior products $u_{i_{1}} \wedge \cdots \wedge u_{i_{k}}\left(1 \leq i_{1}<\ldots<i_{k} \leq 2 n\right)$. Then $V_{k}$ is a subspace of $T^{k}\left(V_{1}\right)$ and $\varphi\left(V_{k}\right)=V_{k-2}$. Hence $\operatorname{dim} V_{k} \cap$ $\operatorname{Ker} \varphi=\left(\begin{array}{c}2 n \\ k\end{array}\right)-\left(\begin{array}{c}2 n \\ k-2\end{array}\right)$. As we saw in Example 21, $V_{k}$ is a representation space of $\mathfrak{Z}(2 n, C)$. Since $\mathfrak{g p}(n, C)$ is a Lie subalgebra of $\mathfrak{Z}(2 n, C)$, $V_{k}$ can be considered as a representation space of $\mathfrak{g p}(n, C)$. This is not irreducible, but the subspace $V_{k} \cap \operatorname{Ker} \varphi$ is an irreducible representation space of $\mathfrak{B p}(n, C)$ with the highest weight $\Lambda_{k}=\lambda_{1}+\cdots+\lambda_{k}$, i.e., a fundamental representation of $\mathfrak{g} p(n, C)$. Thus we obtain that $d\left(\Lambda_{\nu}\right)=$ $\left(\begin{array}{c}2 n \\ \nu\end{array}\right)-\left(\begin{array}{c}2 n \\ \nu-2\end{array}\right)$ for $1 \leq \nu \leq n$.

EXAMPLE 26. We shall calculate the representation degrees of $\mathfrak{s p}(n, C)$ for some cases which will be used in $\S 3$. Define the lexicographical order in $\mathfrak{h}_{0}^{*}$ such that $\Pi=\left\{\alpha_{1}, \cdots, \alpha_{n}\right\}$ in Example 25 are simple roots. Then $\Delta_{+}=\left\{2 \lambda_{1}, \cdots, 2 \lambda_{n}, \lambda_{i} \pm \lambda_{j}(1 \leq i<j \leq n)\right\}$ and 


$$
\rho=\frac{1}{2} \sum_{\alpha \in \Delta_{+}} \alpha=\sum_{k=1}^{n}(n+1-k) \lambda_{k} .
$$

The inner product in $\mathfrak{h}_{0}^{*}$ is given by

$$
\left(\sum_{i=1}^{n} m_{i} \lambda_{i}, \sum_{j=1}^{n} n_{j} \lambda_{j}\right)=\frac{1}{4(n+1)} \sum_{k=1}^{n} m_{k} n_{k} \cdot
$$

Define another product $\{$,$\} by \left\{\sum m_{i} \lambda_{i}, \sum n_{j} \lambda_{j}\right\}=\sum m_{k} n_{k}$. Then the Weyl's dimension formula (Theorem 12) says that

$$
d(\Lambda)=\prod_{\alpha \in \Delta_{+}} \frac{(\Lambda+\rho, \alpha)}{(\rho, \alpha)}=\prod_{\alpha \in \Delta_{+}} \frac{\{\Lambda+\rho, \alpha\}}{\{\rho, \alpha\}} .
$$

Assume that $\Lambda=m_{1} \lambda_{1}+m_{2} \lambda_{2}$ with $m_{1} \geqq m_{2} \geqq 0$. Then $\left(\Lambda, 2 \lambda_{i}\right)=0(3 \leq$ $i \leq n),\left(\Lambda, \lambda_{i} \pm \lambda_{j}\right)=0(3 \leq i<j \leq n)$ and hence we obtain that

$$
\begin{aligned}
d(\Lambda)= & \frac{\left\{\Lambda+\rho, 2 \lambda_{1}\right\}\left\{\Lambda+\rho, 2 \lambda_{2}\right\}\left\{\Lambda+\rho, \lambda_{1}+\lambda_{2}\right\}\left\{\Lambda+\rho, \lambda_{1}-\lambda_{2}\right\}}{\left\{\rho, 2 \lambda_{1}\right\}\left\{\rho, 2 \lambda_{2}\right\}\left\{\rho, \lambda_{1}+\lambda_{2}\right\}\left\{\rho, \lambda_{1}-\lambda_{2}\right\}} \\
& \times \prod_{i=1}^{2} \prod_{j=3}^{n} \frac{\left\{\Lambda+\rho, \lambda_{i}+\lambda_{j}\right\}\left\{\Lambda+\rho, \lambda_{i}-\lambda_{j}\right\}}{\left\{\rho, \lambda_{i}+\lambda_{j}\right\}\left\{\rho, \lambda_{i}-\lambda_{j}\right\}} .
\end{aligned}
$$

Here $\left\{\rho, \lambda_{i}+\lambda_{j}\right\}=2 n+2-i-j(1 \leq i \leq j \leq n),\left\{\rho, \lambda_{i}-\lambda_{j}\right\}=j-i(1 \leq$ $i<j \leq n), \quad\left\{\Lambda, 2 \lambda_{i}\right\}=2 m_{i}(i=1,2), \quad\left\{\Lambda, \lambda_{1} \pm \lambda_{2}\right\}=m_{1} \pm m_{2}$, and $\left\{\Lambda, \lambda_{i} \pm \lambda_{j}\right\}$ $=m_{i}(i=1,2, j=3, \cdots, n)$. Thus we obtain that

$$
\begin{aligned}
& d\left(m_{1} \lambda_{1}+m_{2} \lambda_{2}\right) \\
& =\frac{\left(2 n-2+m_{1}\right) !\left(2 n-3+m_{2}\right) !\left(2 n-1+m_{1}+m_{2}\right)\left(1+m_{1}-m_{2}\right)}{(2 n-1) !(2 n-3) !\left(m_{1}+1\right) ! m_{2} !} .
\end{aligned}
$$

In particular, we get

(1) $d\left(m \Lambda_{1}\right)=\frac{(2 n-1+m) !}{(2 n-1) ! m !}$ (i.e., $\left.m_{1}=m, m_{2}=0\right)$

(i ) $d\left(\Lambda_{1}\right)=2 n$

(ii) $d\left(2 \Lambda_{1}\right)=n(2 n+1)=\operatorname{dim} \mathfrak{s p}(n, C)$

(iii) $d\left(3 \Lambda_{1}\right)=\frac{2}{3} n(n+1)(2 n+1)$

(2) $d\left(\Lambda_{1}+\Lambda_{2}\right)=\frac{8}{3} n\left(n^{2}-1\right)$ (i.e., $m_{1}=2, m_{2}=1$ )

(3) $d\left(2 \Lambda_{2}\right)=\frac{1}{3} n(n-1)(2 n-1)(2 n+3)$ (i.e., $\left.m_{1}=m_{2}=2\right)$

(4) $d\left(\Lambda_{2}\right)=(n-1)(2 n+1)=\left(\begin{array}{c}2 n \\ 2\end{array}\right)-\left(\begin{array}{c}2 n \\ 0\end{array}\right)$ (i.e., $m_{1}=m_{2}=1$ )

This is a special case of $d\left(\Lambda_{\nu}\right)=\left(\begin{array}{c}2 n \\ \nu\end{array}\right)-\left(\begin{array}{c}2 n \\ \nu-2\end{array}\right)$ in Example 25.

Now assume that $n=3$ and $\Lambda=m_{1} \lambda_{1}+m_{2} \lambda_{2}+m_{3} \lambda_{3}$ with $m_{1} \geqq m_{2} \geqq$ 
$m_{3} \geqq 0$. Then $\Delta_{+}=\left\{2 \lambda_{1}, 2 \lambda_{2}, 2 \lambda_{3}, \lambda_{1} \pm \lambda_{2}, \lambda_{1} \pm \lambda_{3}, \lambda_{2} \pm \lambda_{3}\right\}$ and $\rho=3 \lambda_{1}+2 \lambda_{2}+\lambda_{3}$. Since $\left\{\rho, \lambda_{i}+\lambda_{j}\right\}=8-i-j,\left\{\rho, \lambda_{i}-\lambda_{j}\right\}=j-i,\left\{\Lambda, \lambda_{i} \pm \lambda_{j}\right\}=m_{i} \pm m_{j}$, we obtain that $d(\Lambda)=\prod_{\alpha \in \Delta_{+}} \frac{\{\Lambda+\rho, \alpha\}}{\{\rho, \alpha\}}=\frac{1}{5760}\left(6+2 m_{1}\right)\left(4+2 m_{2}\right)\left(2+2 m_{3}\right)$ $\left(5+m_{1}+m_{2}\right)\left(4+m_{1}+m_{3}\right)\left(3+m_{2}+m_{3}\right)\left(1+m_{1}-m_{2}\right)\left(1+m_{2}-m_{3}\right)$ $\left(2+m_{1}-m_{3}\right)$. In particular,

(5) $d\left(\Lambda_{1}+\Lambda_{3}\right)=70\left(n=3, m_{1}=2, m_{2}=m_{3}=1\right)$

(6) $d\left(\Lambda_{2}+\Lambda_{3}\right)=126\left(n=3, m_{1}=m_{2}=2, m_{3}=1\right)$

(7) $d\left(2 \Lambda_{3}\right)=84\left(n=3, m_{1}=m_{2}=m_{3}=2\right)$.

EXAMPle 27. Let $\mathfrak{o}(n, C)=\left\{\left.X \in \mathfrak{g l}(n, C)\right|^{t} X+X=0\right\}$ be a Lie subalgebra of $\mathfrak{g l}(n, C)$. The Killing form $B$ of $\mathfrak{o}(n, C)$ is given by $B(X, Y)$ $=\operatorname{Trad} X \operatorname{ad} Y=(n-2) \operatorname{Tr} X Y$. If an element $X$ of $\mathfrak{o}(n, C)$ satisfies the condition $B(X, A)=0$ for any $A \in \mathfrak{v}(n, C)$, then $X={ }^{t} X$ since $\operatorname{Tr}\left(X-{ }^{t} X\right) Z$ $=\operatorname{Tr} X\left(Z-{ }^{t} Z\right)=0$ for any $Z \in \mathfrak{g l}(n, C)$. On the other hand, as $X \in \mathfrak{v}(n, C)$ implies $X=-{ }^{t} X$, and we get that $X=0$. This shows that $B$ is non-degenerate and hence $\mathfrak{o}(n, C)$ is semi-simple. First, we shall consider the case of $n=2 m+1$. Put

$$
K=\left(\begin{array}{c|c|c|c|c|c}
1 & \multicolumn{2}{|c|}{0} \\
\hline 0 & 0 & I_{m} \\
\hline & I_{m} & 0
\end{array}\right], \quad T=\left(\begin{array}{c|c|c}
1 & \multicolumn{2}{|c}{0} \\
\hline 0 & \frac{1}{\sqrt{2}} I_{m} & \frac{\sqrt{-1}}{\sqrt{2}} I_{m} \\
\hline \frac{1}{\sqrt{2}} I_{m} & \frac{-\sqrt{-1}}{\sqrt{2}} I_{m}
\end{array}\right) .
$$

Let $\mathfrak{g}=\left\{A \in \operatorname{gr}(2 m+1, C) \mid{ }^{t} A K+K A=0\right\}$ be a Lie subalgebra of $\mathfrak{g l}(2 m+1, C)$. Since $T^{-1} \mathfrak{g} T=\mathfrak{o}(2 m+1, C), \mathfrak{g}$ is isomorphic to $\mathfrak{o}(2 m+1, C)$ over $C$ and sometimes we denote $\mathfrak{g}$ also by $\mathfrak{b}(2 m+1, C)$. $\mathfrak{g}$ is the totality of elements of $\operatorname{gr}(2 m+1, C)$ of the form:

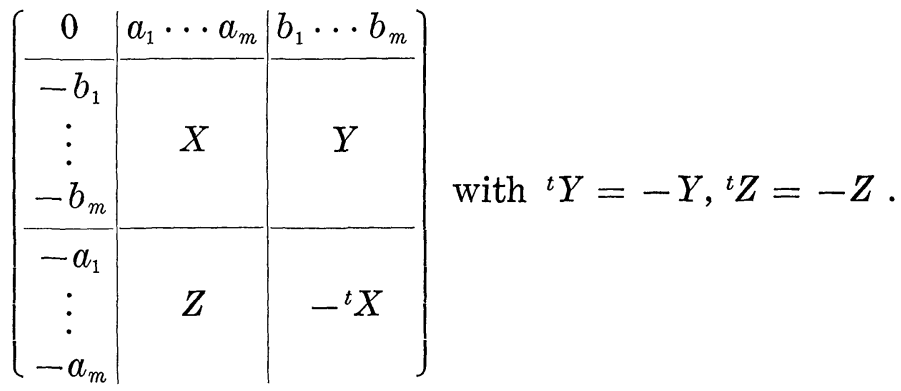

Denote by $H=H\left(\lambda_{1}, \cdots, \lambda_{m}\right)$ the element of $g$ such that $X$ is a 
diagonal matrix with diagonal elements $\lambda_{1}, \cdots, \lambda_{m}$ and $Y=Z=0, a_{i}=$ $b_{i}=0(1 \leq i \leq m)$ in (1.4). The totality $\mathfrak{h}$ of such elements is a Cartan subalgebra of $g$, and $\Delta=\left\{ \pm \lambda_{i}, \pm \lambda_{j} \pm \lambda_{k}(j<k)\right\}$ is the root system of g.w.r.t.h. Put $\alpha_{1}=\lambda_{1}-\lambda_{2}, \alpha_{2}=\lambda_{2}-\lambda_{3}, \cdots, \alpha_{m-1}=\lambda_{m-1}-\lambda_{m}, \alpha_{m}=\lambda_{m}$. Then $\pi=\left\{\alpha_{1}, \cdots, \alpha_{m}\right\}$ is a fundamental root system because $\lambda_{i}-\lambda_{j}=\alpha_{i}$ $+\alpha_{i+1}+\cdots+\alpha_{j}$ for $i<j, \lambda_{i}-\lambda_{j}=-\left(\alpha_{j}+\alpha_{j+1}+\cdots+\alpha_{i}\right)$ for $i>j, \pm \lambda_{i}$ $= \pm\left(\left(\lambda_{i}-\lambda_{m}\right)+\lambda_{m}\right)$, and $\pm\left(\lambda_{i}+\lambda_{j}\right)= \pm\left(\left(\lambda_{i}-\lambda_{m}\right)+\left(\lambda_{j}-\lambda_{m}\right)+2 \lambda_{m}\right)$. As

$$
H_{\alpha_{i}}=\frac{1}{2(2 m-1)} H(0, \ldots, \stackrel{i}{1}, \stackrel{i+1}{1}, 0, \ldots, 0)
$$

for $1 \leq i \leq m-1$ and $H_{\alpha_{m}}=\frac{1}{2(2 m-1)} H(0, \cdots, 0,1)$, we obtain that $\left(\alpha_{i}, \alpha_{j}\right)=0$ for $|i-j| \geqq 2,\left(\alpha_{i}, \alpha_{j}\right)=-\frac{1}{2(2 m-1)}$ for $|i-j|=1,\left(\alpha_{i}, \alpha_{i}\right)=$ $\frac{1}{2 m-1}$ for $1 \leq i \leq m-1$ and $\left(\alpha_{m}, \alpha_{m}\right)=\frac{1}{2(2 m-1)}$. This shows that $\mathfrak{g}=\mathfrak{o}(2 m+1, C)$ is a simple Lie algebra of type $B_{m}$. The fundamental dominant weights are $\Lambda_{1}=\lambda_{1}, \Lambda_{2}=\lambda_{1}+\lambda_{2}, \cdots, \Lambda_{m-1}=\lambda_{1}+\cdots+\lambda_{m-1}$, $\Lambda_{m}=\frac{1}{2}\left(\lambda_{1}+\cdots+\lambda_{m}\right)$. If $m=1$, then $\Lambda_{1}=\frac{1}{2} \lambda_{1}$. Let $V_{1}$ be a $n(=2 m+$ 1)-dimensional vector space over $C$ spanned by $u_{1}, \cdots, u_{n}$. For $1 \leq k \leq$ $m-1$, let $V_{k}$ be a $\left(\begin{array}{l}n \\ k\end{array}\right)$-dimensional vector space over $C$ spanned by exterior products $u_{i_{1}} \wedge \cdots \wedge u_{i_{k}}\left(1 \leq i_{1}<\cdots<i_{k} \leq n\right)$. Then as we saw in Example 21, $V_{k}$ is an irreducible representation space of $\operatorname{gr}(n, C)$ and its restriction to $\mathfrak{o}(n, C)$ is still irreducible. Thus $V_{k}$ is an irreducible representation space of $\mathfrak{D}(n, C)$ with the highest weight $\Lambda_{k}(1 \leq k \leq m-1)$. Hence $d\left(\Lambda_{k}\right)=\left(\begin{array}{l}n \\ k\end{array}\right)=\left(\begin{array}{c}2 m+1 \\ k\end{array}\right)$ for $1 \leq k \leq m-1$.

Now we shall calculate $d\left(\Lambda_{m}\right)$. The inner product in $\mathfrak{h}_{0}^{*}$ is

$$
\left(\sum m_{i} \lambda_{i}, \sum n_{j} \lambda_{j}\right)=\frac{1}{2(2 m-1)} \sum_{k=1}^{m} m_{k} n_{k} .
$$

Define the inner product $\{$,$\} by \left\{\sum m_{i} \lambda_{i}, \sum n_{j} \lambda_{j}\right\}=\sum m_{k} n_{k}$. Under the lexicographical order in $\mathfrak{h}_{0}^{*}$ such that $\Pi=\left\{\alpha_{1}, \cdots, \alpha_{m}\right\}$ are simple roots, $\Delta_{+}=\left\{\lambda_{1}, \cdots, \lambda_{m}, \lambda_{i} \pm \lambda_{j}(1 \leq i<j \leq m)\right\}$ and $\rho=\sum_{k=1}^{m}\left(m-k+\frac{1}{2}\right) \lambda_{k}$. Since $\Lambda_{m}=\frac{1}{2}\left(\lambda_{1}+\cdots+\lambda_{m}\right),\left\{\Lambda_{m}, \lambda_{i}-\lambda_{j}\right\}=0,\left\{\Lambda_{m}, \lambda_{i}+\lambda_{j}\right\}=1$, and $\left\{\Lambda_{m}, \lambda_{i}\right\}=\frac{1}{2}$, the Weyl's dimension formula says that 


$$
\begin{aligned}
d\left(\Lambda_{m}\right) & =\prod_{\alpha \in \Delta_{+}} \frac{\left(\Lambda_{m}+\rho, \alpha\right)}{(\rho, \alpha)}=\prod_{i=1}^{m} \frac{\left\{\Lambda_{m}+\rho, \lambda_{i}\right\}}{\left\{\rho, \lambda_{i}\right\}} \prod_{1 \leq i<j \leq m} \frac{\left\{\Lambda_{m}+\rho, \lambda_{i}+\lambda_{j}\right\}}{\left\{\rho, \lambda_{i}+\lambda_{j}\right\}} \\
& =\prod_{i=1}^{m} \frac{(m-i+1)}{\left(m-i+\frac{1}{2}\right)} \prod_{1 \leq i<j \leq m} \frac{(2 m+2-i-j)}{(2 m+1-i-j)} .
\end{aligned}
$$

Here

$$
\begin{aligned}
& \prod_{1 \leq i<j \leq m} \frac{(2 m+2-i-j)}{(2 m+1-i-j)} \\
= & \frac{\prod_{i=1}^{m-1}(2 m+2-i-(i+1)) \prod_{2 \leq j-i}(2 m+2-i-j)}{\prod_{i=1}^{m-1}(2 m+1-i-m) \prod_{1 \leq i<j<m}(2 m+1-i-j)} \\
= & \prod_{i=1}^{m-1} \frac{(2 m+2-i-(i+1))}{(2 m+1-i-m)}=2^{m-1} \prod_{i=1}^{m-1} \frac{\left(m-i+\frac{1}{2}\right)}{(m-i+1)},
\end{aligned}
$$

and hence we get $d\left(\Lambda_{m}\right)=2^{m}=2^{(n-1) / 2}$. This $2^{m}$-dimensional representation of $\mathrm{g}=\mathfrak{o}(2 m+1, C)$ is called the spin representation. We shall construct the spin representation in $\S 5$. Finally, we shall calculate $d\left(s \Lambda_{1}\right)$.

By the Weyl's dimension formula,

$$
\begin{aligned}
d\left(s \Lambda_{1}\right) & =\frac{\left(s \Lambda_{1}+\rho, \lambda_{1}\right)}{\left(\rho, \lambda_{1}\right)} \prod_{j=2}^{m} \frac{\left(s \Lambda_{1}+\rho, \lambda_{1}+\lambda_{j}\right)\left(s \Lambda_{1}+\rho, \lambda_{1}-\lambda_{j}\right)}{\left(\rho, \lambda_{1}+\lambda_{j}\right)\left(\rho, \lambda_{1}-\lambda_{j}\right)} \\
& =\frac{\left(m-\frac{1}{2}+s\right)}{\left(m-\frac{1}{2}\right)} \prod_{j=2}^{m} \frac{(2 m-j+s)(j-1+s)}{(2 m-j)(j-1)} \\
& =\frac{(2 m+s-2) !(2 m+2 s-1)}{(2 m-1) ! s !} .
\end{aligned}
$$

Thus we obtain the following results.

(1) $d\left(\Lambda_{\nu}\right)=\left(\begin{array}{l}n \\ \nu\end{array}\right)$ for $1 \leq \nu \leq m-1(n=2 m+1)$

(2) $d\left(\Lambda_{m}\right)=2^{m}=2^{(n-1) / 2}(n=2 m+1)$

(3) $d\left(s \Lambda_{1}\right)=\frac{(2 m+s-2) !(2 m+2 s-1)}{(2 m-1) ! s !}$, in particular

(3') $d\left(2 \Lambda_{1}\right)=m(2 m+3)=\frac{1}{2}(n-1)(n+2)(n=2 m+1)$.

EXAMPLE 28. We shall consider the Lie algebra $\mathfrak{o}(n, C)$ with $n=2 m$. Put

$$
K=\left(\begin{array}{c|c}
0 & I_{m} \\
\hline I_{m} & 0
\end{array}\right], \quad T=\left(\begin{array}{c|c}
\frac{1}{\sqrt{2}} I_{m} & \frac{\sqrt{-1}}{\sqrt{2}} I_{m} \\
\hline \frac{1}{\sqrt{2}} I_{m} & -\frac{\sqrt{-1}}{\sqrt{2}} I_{m}
\end{array}\right]
$$


$\mathfrak{g}=\left\{X \in \mathfrak{g l}(2 m, C) \mid{ }^{t} A K+K A=0\right\}$. Then $\mathfrak{g}$ is isomorphic to $\mathfrak{o}(2 m, C)$ over $C$ since $\mathfrak{o}(2 m, C)=T^{-1} \mathrm{~g} T$. One can easily check that

$$
\mathrm{g}=\left\{\left(\frac{X}{Z} \mid \frac{Y}{-{ }^{t} X}\right) \mid{ }^{t} Y=-Y,{ }^{t} Z=-Z\right\}
$$

and the totality $\mathfrak{h}$ of elements $H=H\left(\lambda_{1}, \cdots, \lambda_{n 1}\right)$ of $g$ such that $X$ is a diagonal matrix with diagonal elements $\lambda_{1}, \cdots, \lambda_{m}$ and $Y=Z=0$ in (1.6) is a Cartan subalgebra of $\mathfrak{g}$. The root system $\Delta$ of $\mathfrak{g}$ w.r.t. $\mathfrak{h}$ is given by $\Delta=\left\{\lambda_{i} \pm \lambda_{k}(i \neq k)\right\}$ and $\Pi=\left\{\alpha_{1}=\lambda_{1}-\lambda_{2}, \cdots, \alpha_{m-1}=\lambda_{m-1}-\lambda_{m}, \alpha_{m}=\right.$ $\left.\lambda_{m-1}+\lambda_{m}\right\}$ is a fundamental root system because $\lambda_{i}-\lambda_{j}=\alpha_{i}+\alpha_{i+1}+$ $\cdots+\alpha_{j}$ for $i<j, \lambda_{i}-\lambda_{j}=-\left(\alpha_{j}+\alpha_{j+1}+\cdots+\alpha_{i-1}\right)$ if $i>j$, and $\pm\left(\lambda_{i}+\right.$ $\left.\lambda_{j}\right)= \pm\left(\left(\lambda_{i}-\lambda_{m-1}\right)+\left(\lambda_{j}-\lambda_{m}\right)+\left(\lambda_{m-1}+\lambda_{m}\right)\right)$ for $i<j$. As $H_{\alpha_{i}}=$ $\frac{1}{2(2 m-2)} H(0, \ldots, \stackrel{i}{1}, \stackrel{i+1}{-1}, \ldots, 0) \quad(1 \leq i \leq m-1)$ and $H_{\alpha_{m}}=\frac{1}{2(2 m-2)}$ $H(0, \cdots, 0,1,1)$, we obtain that

$$
\left(\alpha_{i}, \alpha_{j}\right)=\left\{\begin{array}{cl}
0 & (|i-j| \geqq 2, i \leq m-1, j \leq m-1) \\
\frac{-1}{2(2 m-2)}(i=m-2, j=m) \\
\frac{-1}{2(2 m-2)}(|i-j|=1, i \leq m-1, j \leq m-1) \\
0 \quad(i \neq m-2, j=m) \\
\frac{1}{(2 m-2)}(i=j) .
\end{array}\right.
$$

If $m=2$, then $\Pi=\left\{\alpha_{1}, \alpha_{2}\right\},\left\{\alpha_{1}\right\} \perp\left\{\alpha_{2}\right\}$ and this implies that $\mathfrak{o}(4, C)$ is not simple. In fact, $\mathfrak{0}(4, C) \cong \mathfrak{I l}(2, C) \oplus \mathfrak{I l}(2, C)$. If $m \geqq 3$, this shows that $\mathrm{g}(\cong \mathrm{o}(2 m, C))$ is a simple Lie algebra of type $D_{m}$. The fundamental dominant weights are $\Lambda_{\nu}=\lambda_{1}+\cdots+\lambda_{\nu}(1 \leq \nu \leq m-2), \Lambda_{m-1}=\frac{1}{2}\left(\lambda_{1}+\cdots+\right.$ $\left.\lambda_{m-1}-\lambda_{m}\right)$, and $\Lambda_{m}=\frac{1}{2}\left(\lambda_{1}+\cdots+\lambda_{m}\right)$. Similarly as in Example 27, we can calculate the following results.

(1) $d\left(\Lambda_{\nu}\right)=\left(\begin{array}{l}n \\ \nu\end{array}\right)=\left(\begin{array}{c}2 m \\ \nu\end{array}\right)$ for $1 \leq \nu \leq m-2(n=2 m)$

(2) $d\left(\Lambda_{m-1}\right)=d\left(\Lambda_{m}\right)=2^{m-1}=2^{n / 2-1}(n=2 m)$

(3) $d\left(2 \Lambda_{1}\right)=(2 m-1)(m+1)=\frac{1}{2}(n-1)(n+2)(n=2 m)$.

Here $\Lambda_{2}$ is the adjoint representation (see Example 24 (7)).

The irreducible representation $d \rho_{\nu}$ with the highest weight $\Lambda_{\nu}(\nu=$ $m-1$, or $m$ ) is called the even (resp. odd) half-spin representation of 
$\mathfrak{g}=\mathfrak{o}(2 m, C)$ if $\nu$ is even (resp. odd). Although $d \rho_{m-1}$ and $d \rho_{m}$ are not equivalent, there exists an outer automorphism $\sigma$ of $\mathfrak{g}$ such that $d \rho_{m-1} \circ \sigma$ and $d \rho_{m}$ are equivalent. The restrictions of $d \rho_{m-1}$ and $d \rho_{m}$ to $\mathfrak{o}(2 m-1, C)$ are equivalent and it is the spin representation of $\mathrm{o}(2 m-1, C)$. In the case of $m=4$, we have $d\left(\Lambda_{1}\right)=d\left(\Lambda_{3}\right)=d\left(\Lambda_{4}\right)=8$, and moreover there exist outer automorphisms $\sigma_{3}, \sigma_{4}$ such that $d \rho_{1}, d \rho_{3} \circ \sigma_{3}, d \rho_{4} \circ \sigma_{4}$ are equivalent, where $d \rho_{1}$ denotes the standard representation of $\mathfrak{o}(8, C)$ with the highest weight $\Lambda_{1}$ (see (5.30) in $\S 5$ ). The weights of $d \rho_{1}, d \rho_{3}, d \rho_{4}$ are $\left\{ \pm \lambda_{i}\right.$, $i=1,2,3,4\},\left\{ \pm \Lambda_{i}^{\prime}, i=1,2,3,4\right\},\left\{ \pm \Lambda_{i}^{*}, i=1,2,3,4\right\}$ respectively, where $\Lambda_{i}^{\prime}=\frac{1}{2}\left(\lambda_{1}+\lambda_{2}+\lambda_{3}+\lambda_{4}\right)-\lambda_{i}, \Lambda_{1}^{*}=\frac{1}{2}\left(\lambda_{1}+\lambda_{2}+\lambda_{3}+\lambda_{4}\right), \Lambda_{2}^{*}=\frac{1}{2}\left(\lambda_{1}+\lambda_{2}-\lambda_{3}-\lambda_{4}\right)$, $\Lambda_{3}^{*}=\frac{1}{2}\left(\lambda_{1}-\lambda_{2}+\lambda_{3}-\lambda_{4}\right), \Lambda_{4}^{*}=\frac{1}{2}\left(\lambda_{1}-\lambda_{2}-\lambda_{3}+\lambda_{4}\right)$. We shall construct the even half-spin representation in $\S 5$.

DEFINITION 29. Let $d \rho: \mathfrak{g} \rightarrow \mathfrak{g l}(V)$ be a representation of a Lie algebra $\mathfrak{g}$ on $V$. Let $x$ be an element of $V$ and let $\mathfrak{g}_{x}=\{A \in \mathfrak{g} \mid d \rho(A) x=0\}$ be a subset of $\mathrm{g}$. Then $\mathrm{g}_{x}$ is a subalgebra of $\mathrm{g}$ and is called the isotropy subalgebra of $g$ at $x$.

EXAMPLE 30. Let $g$ be the totality of elements of $\operatorname{gr}(7, C)$ of the form :

$$
A=\left(\begin{array}{c|ccc|ccc}
0 & 2 d & 2 e & 2 f & 2 a & 2 b & 2 c \\
\hline a & & & & 0 & f & -e \\
b & & X & & -f & 0 & d \\
c & & & & e & -d & 0
\end{array}\right) \quad \text { with } X \in \mathfrak{g l}(3, C)
$$

Then $\mathfrak{g}$ is a Lie subalgebra of $\operatorname{gr}(7, C)$. In fact, this is the isotropy subalgebra of $\operatorname{gr}(7, C)$ at $x=u_{2} \wedge u_{3} \wedge u_{4}+u_{5} \wedge u_{6} \wedge u_{7}+u_{1} \wedge\left(u_{2} \wedge u_{5}+\right.$ $u_{3} \wedge u_{6}+u_{4} \wedge u_{7}$ ) under the irreducible representation with the highest weight $\Lambda_{3}(=-$ - (see Example 21 and (8) in $\S 5$ ).

The Killing form $B$ of $\mathrm{g}$ is $B\left(A, A^{\prime}\right)=\operatorname{Tr}$ ad $A$ ad $A^{\prime}=24\left(a d^{\prime}+a^{\prime} d\right.$ $\left.+b e^{\prime}+b^{\prime} e+c f^{\prime}+c^{\prime} f\right)+8 \operatorname{Tr} X X^{\prime}$, and as this is non-degenerate, $g$ is a semi-simple Lie algebra. The totality $\mathfrak{h}$ of elements $H=H\left(\lambda_{1}, \lambda_{2}\right)$ of $\mathfrak{g}$ such that $X$ is a diagonal matrix with diagonal element $\lambda_{1}, \lambda_{2}, \lambda_{3}=-\lambda_{1}-\lambda_{2}$, all remaining entries zero in (1.8), is a Cartan subalgebra of $\mathfrak{g}$.

The root system $\Delta$ of $g$ w.r.t. $\mathfrak{h}$ is given by 


$$
\Delta=\left\{ \pm \lambda_{1}, \pm \lambda_{2}, \pm \lambda_{1} \pm \lambda_{2}, \pm\left(\lambda_{1}+2 \lambda_{2}\right), \pm\left(2 \lambda_{1}+\lambda_{2}\right)\right\}
$$

A subset $\Pi=\left\{\alpha_{1}=\lambda_{1}-\lambda_{2}, \alpha_{2}=\lambda_{2}\right\}$ of $\Delta$ is a fundamental root system since $\pm \lambda_{1}= \pm\left(\alpha_{1}+\alpha_{2}\right), \pm \lambda_{2}= \pm \alpha_{2}, \pm\left(\lambda_{1}+\lambda_{2}\right)= \pm\left(\alpha_{1}+2 \alpha_{2}\right), \pm\left(\lambda_{1}-\lambda_{2}\right)=$ $\pm \alpha_{1}, \pm\left(\lambda_{1}+2 \lambda_{2}\right)= \pm\left(\alpha_{1}+3 \alpha_{2}\right), \pm\left(2 \lambda_{1}+\lambda_{2}\right)= \pm\left(2 \alpha_{1}+3 \alpha_{2}\right)$. Since $B\left(H, H^{\prime}\right)$ $=8\left(2 \lambda_{1}+\lambda_{2}\right) \lambda_{1}^{\prime}+8\left(\lambda_{1}+2 \lambda_{2}\right) \lambda_{2}^{\prime}$, we obtain that

$$
H_{m_{1} \lambda_{1}+m_{2} \lambda_{2}}=H\left(\frac{2 m_{1}-m_{2}}{24}, \frac{2 m_{2}-m_{1}}{24}\right)
$$

and $\left(m_{1} \lambda_{1}+m_{2} \lambda_{2}, n_{1} \lambda_{1}+n_{2} \lambda_{2}\right)=\frac{1}{12}\left(m_{1} n_{1}+m_{2} n_{2}\right)-\frac{1}{24}\left(m_{1} n_{2}+m_{2} n_{1}\right)$, in particular, $\left(\alpha_{1}, \alpha_{1}\right)=\frac{1}{4},\left(\alpha_{1}, \alpha_{2}\right)=-\frac{1}{8},\left(\alpha_{2}, \alpha_{2}\right)=\frac{1}{12}$, i.e., $\left\|\alpha_{1}\right\|=\sqrt{3}\left\|\alpha_{2}\right\|, \widehat{\alpha_{1} \alpha_{2}}=\frac{5}{6} \pi$.

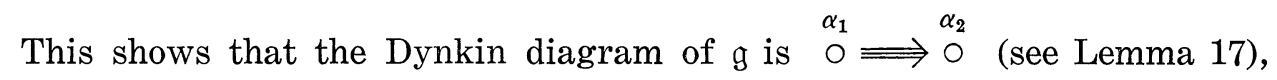
and hence $g$ is a simple Lie algebra of type $G_{2}$.

We sometimes denote $\mathfrak{g}$ by $\mathfrak{g}_{2}$ or $\left(\mathfrak{g}_{2}\right)$. Now we shall calculate the fundamental dominant weights $\Lambda_{1}=m_{1} \lambda_{1}+m_{2} \lambda_{2}$ and $\Lambda_{2}=n_{1} \lambda_{1}+n_{2} \lambda_{2}$. Since $\frac{2\left(\Lambda_{1}, \alpha_{1}\right)}{\left(\alpha_{1}, \alpha_{1}\right)}=m_{1}-m_{2}=1$ and $\left(\Lambda_{1}, \alpha_{2}\right)=\frac{1}{24}\left(2 m_{2}-m_{1}\right)=0$, we obtain that $m_{1}=2, m_{2}=1$, i.e., $\Lambda_{1}=2 \lambda_{1}+\lambda_{2}\left(=2 \alpha_{1}+3 \alpha_{2}\right)$. Similarly as $\left(\Lambda_{2}, \alpha_{1}\right)$ $=\frac{1}{8}\left(n_{1}-n_{2}\right)=0$ and $\frac{2\left(\Lambda_{2}, \alpha_{2}\right)}{\left(\alpha_{2}, \alpha_{2}\right)}=2 n_{2}-n_{1}=1$, we obtain that $n_{1}=n_{2}$ $=1$, i.e., $\Lambda_{2}=\lambda_{1}+\lambda_{2}\left(=\alpha_{1}+2 \alpha_{2}\right)$. Define the lexicographical order in $\mathfrak{h}_{0}^{*}$ such that $I I=\left\{\alpha_{1}, \alpha_{2}\right\}$ are simple roots. Then $\Delta_{+}=\left\{\alpha_{1}, \alpha_{2}, \alpha_{1}+\alpha_{2}, \alpha_{1}+\right.$ $\left.2 \alpha_{2}, \alpha_{1}+3 \alpha_{2}, 2 \alpha_{1}+3 \alpha_{2}\right\}$ and $\rho=\frac{1}{2} \sum_{\alpha \in \Delta_{+}} \alpha=3 \alpha_{1}+5 \alpha_{2}$. For a dominant integral form $\Lambda=m_{1} \Lambda_{1}+m_{2} \Lambda_{2}$, since $\left(\Lambda+\rho, \alpha_{1}\right)=\frac{m_{1}+1}{8},\left(\Lambda+\rho, \alpha_{2}\right)=$ $\frac{m_{2}+1}{24},\left(\rho, \alpha_{1}\right)=\frac{1}{8}$, and $\left(\rho, \alpha_{2}\right)=\frac{1}{24}$, the Weyl's dimension formula says that $d(\Lambda)=\prod_{\alpha \in A_{+}} \frac{(\Lambda+\rho, \alpha)}{(\rho, \alpha)}=\frac{1}{120}\left(m_{1}+1\right)\left(m_{2}+1\right)\left(m_{1}+m_{2}+2\right)\left(2 m_{1}+m_{2}\right.$ $+3)\left(3 m_{1}+m_{2}+4\right)\left(3 m_{1}+2 m_{2}+5\right)$. In particular, $d\left(\Lambda_{2}\right)=7, d\left(\Lambda_{1}\right)=14$, $d\left(2 \Lambda_{2}\right)=27, d\left(\Lambda_{1}+\Lambda_{2}\right)=64, d\left(2 \Lambda_{1}\right)=d\left(3 \Lambda_{2}\right)=77, d\left(2 \Lambda_{1}+\Lambda_{2}\right)=189 . \quad$ In view of Corollary $13,14\left(=\operatorname{dim}\left(\mathrm{g}_{2}\right)\right.$ )-dimensional representation is only $\Lambda_{1}$, and hence $\Lambda_{1}$ must be the adjoint representation of $\left(\mathfrak{g}_{2}\right)$. The identity map of $\mathrm{g}$ into $\mathrm{gr}(7, C)$ is $\Lambda_{2}$. (We denote the representation corresponding to $\Lambda$, by $\Lambda$. See Theorem 10.) The derivation algebra of the Cayley numbers is also $\left(\mathfrak{g}_{2}\right)$ (see Example 41 ).

Now we shall construct the exceptional Lie algebras of type $F_{4}$ and $E_{6}$. 
DeFINITION 31. Let $Q=C \cdot 1+C \cdot e_{1}+C \cdot e_{2}+C \cdot e_{1} e_{2}\left(e_{1}^{2}=e_{2}^{2}=-1\right.$, $\left.e_{1} e_{2}=-e_{2} e_{1}\right)$ be the quaternion algebra over $C$. For a $Q$-module $\mathfrak{c}=$ $Q+Q e$, define the multiplication by $(q+r e) \cdot(s+t e)=(q s-\bar{t} r)+(t q$ $+r \bar{s}) e$ where $q, r, s, t \in Q$ and $\bar{s}, \bar{t}$ are conjugates of $s, t$ respectively. Thus we obtain a non-associative algebra $\sqrt{5}$ of dimension 8 , called the Cayley algebra over $C$. The conjugate $\bar{x}$ of $x=q+r e(q, r \in Q)$ is defined by $\bar{x}=\bar{q}-r e$. Then $\overline{x y}=\bar{y} \cdot \bar{x}$. The exceptional simple Jordan algebra $\mathscr{J}$ over $C$ is the non-associative algebra of dimension 27 whose elements are $3 \times 3$ Hermitian matrices with elements in the Cayley algebra $\mathfrak{}$, multiplication being defined by $X \circ Y=\frac{1}{2}(X Y+Y X)$ where $X Y$ is the ordinary matrix product.

$$
\mathscr{J}=\left\{X=\left(\begin{array}{ccc}
\xi_{1} & x_{3} & \bar{x}_{2} \\
\bar{x}_{3} & \xi_{2} & x_{1} \\
x_{2} & \bar{x}_{1} & \xi_{3}
\end{array}\right) \mid \begin{array}{l}
\xi_{1}, \xi_{2}, \xi_{3} \in C \\
x_{1}, x_{2}, x_{3} \in \mathfrak{S}
\end{array}\right\}
$$

We write the trace $\xi_{1}+\xi_{2}+\xi_{3}=\operatorname{Tr} X$. The derivation algebra $\mathscr{D}$ of $\mathscr{J}$ is the Lie algebra of endomorphisms $\mathrm{D}$ of $\mathscr{J}$ satisfying $D(X \circ Y)=$ $D X \circ Y+X \circ D Y$.

We shall see that $\mathscr{D}$ is a simple Lie algebra of type $F_{4}$. By a right multiplication $R_{Y}$ is meant the endomorphism $X \mapsto X \circ Y$ for every $X$ in $\mathscr{J}$. Then $\left[R_{X}, R_{Y}\right]=R_{X} \circ R_{Y}-R_{Y} \circ R_{X}$ is a derivation of $\mathscr{J}$ and $\left[D, R_{X}\right]$ $=R_{D X}$ for $D \in \mathscr{D}, X \in \mathscr{J}$. Let $\mathfrak{o}(8, C)=\left\{X \in \mathfrak{g}(8, C){ }^{t} X+X=0\right\}$ be the simple Lie algebra $D_{4}$. We regard the elements of $\mathfrak{o}(8, C)$ as endomorphisms of the Cayley algebra 5 . This algebra is equipped with a trace function $\operatorname{tr} x=x+\bar{x}$ satisfying $\operatorname{tr} x y=\operatorname{tr} y x, \operatorname{tr} x(y z)=\operatorname{tr}(x y) z(=\operatorname{tr} x y z)$. Also, tr $x y$ is a non-degenerate bilinear form. An endomorphism $U$ of $\sqrt{ }$ is in $\mathfrak{b}(8, C)$ if and only if $U$ leaves the norm form $x \bar{x}$ invariant:

$$
(U x) \bar{x}+x(\overline{U x})=0 .
$$

Proposition 32 (Principle of Triality). For $U$ in $a(8, C)$, there exist unique $U^{\prime}, U^{\prime \prime}$ in $\mathrm{o}(8, C)$ such that

$$
\operatorname{tr}(U x) y z+\operatorname{tr} x\left(U^{\prime} y\right) z+\operatorname{tr} x y\left(U^{\prime \prime} z\right)=0
$$

for all $x, y, z$ in $\mathfrak{c}^{5}$. These $U^{\prime}$ and $U^{\prime \prime}$ are the inequivalent half-spin representations of $U$ in $D_{4}$ (see Example 28).

Associated with the exceptional simple Jordan algebra $\mathscr{J}$ are the bilinear form $\operatorname{Tr} X \circ Y$ and the trilinear form 


$$
\phi(X, Y, Z)=\operatorname{Tr}(X \circ Y) \circ Z=\operatorname{Tr} X \circ(Y \circ Z) .
$$

Proposition 33. An endomorphism $D$ of $\mathscr{J}$ is a derivation if and only if $D$ leaves both $\operatorname{Tr} X \circ Y$ and $\phi(X, Y, Z)$ invariant, i.e.,

(i) $\operatorname{Tr} D X \circ Y+\operatorname{Tr} X \circ D Y=0$

(ii) $\phi(D X, Y, Z)+\phi(X, D Y, Z)+\phi(X, Y, D Z)=0$.

DEFINITION 34.

$$
\begin{aligned}
E_{1}=\left(\begin{array}{lll}
1 & & \\
& 0 & \\
& & 0
\end{array}\right), & E_{2}=\left(\begin{array}{lll}
0 & & \\
& 1 & \\
& & 0
\end{array}\right), & E_{3}=\left(\begin{array}{lll}
0 & & \\
& 0 & \\
& & 1
\end{array}\right) \\
(a)_{1}=\left(\begin{array}{lll}
0 & & \\
& 0 & \mathrm{a} \\
& \bar{a} & 0
\end{array}\right), & (a)_{2}=\left(\begin{array}{lll}
0 & & \bar{a} \\
& 0 & \\
\mathrm{a} & & 0
\end{array}\right), & (a)_{3}=\left(\begin{array}{lll}
0 & a & \\
\bar{a} & 0 & \\
& & 0
\end{array}\right)
\end{aligned}
$$

for $a \in \mathfrak{E}$.

$$
\begin{aligned}
& \bar{A}_{i}=\left\{\left(a_{i}\right) \in \mathscr{J} \mid a \in \mathfrak{E}\right\} \quad(1 \leq i \leq 3) \\
& \mathscr{D}_{0}=\left\{A \in \mathscr{D} \mid A E_{i}=0 \quad \text { for } 1 \leq i \leq 3\right\}
\end{aligned}
$$

Proposition 35. $\mathscr{D}_{0} \cong \mathrm{v}(8, C), \operatorname{dim} \mathscr{D}_{0}=28$.

Proof. Since $E_{i} \circ(a)_{i}=0$ and $(a)_{i}=2 E_{j} \circ(a)_{i}(j \neq i)$, we obtain that $D(a)_{i}=2 E_{j} \circ D(a)_{i}$ for $D \in \mathscr{D}_{0}, j \neq i$. This implies that $\mathscr{D}_{0} \bar{A}_{i} \subset \bar{A}_{i}$ for $1 \leq i \leq 3$, and let $U, U^{\prime}, U^{\prime \prime}$ be the restriction of $D$ to $\bar{A}_{1}, \bar{A}_{2}, \bar{A}_{3}$ respectively for each $D \in \mathscr{D}_{0}$. As $D$ leaves $\operatorname{Tr}\left(\begin{array}{ccc}0 & & \\ & 0 & x_{1} \\ & \bar{x}_{1} & 0\end{array}\right)^{2}=2 x_{1} \bar{x}_{1}$ invariant, $U$ is an element of $\mathfrak{o}(8, C)$ and so do $U^{\prime}$ and $U^{\prime \prime}$ similarly. Since $D$ leaves the trilinear form $\phi(X, Y, Z)$ invariant, $U, U^{\prime}, U^{\prime \prime}$ satisfy the principle of triality, i.e., $U^{\prime}, U^{\prime \prime}$ are two inequivalent half-spin representations of $U$. Thus we get

$$
X \mapsto D X=\left(\begin{array}{ccc}
0 & U^{\prime \prime} x_{3} & \overline{U^{\prime} x_{2}} \\
\overline{U^{\prime \prime} x_{3}} & 0 & U x_{1} \\
U^{\prime} x_{2} & \overline{U x_{1}} & 0
\end{array}\right) \quad \text { for } D \in \mathscr{D}_{0} .
$$

Conversely, as a linear transformation of this type leaves both $\operatorname{Tr} X \circ Y$ and $\phi(X, Y, Z)$ invariant, it is a derivation of $\mathscr{J}$ by Proposition 33, and hence we obtain our assertion.

Q.E.D. 
DEFINITION 36. $(a)_{1}^{\prime}=\left[R_{E_{2}}, R_{(a)_{1}}\right],(a)_{2}^{\prime}=\left[R_{E_{1}}, R_{(a)_{2}}\right],(a)_{3}^{\prime}=\left[R_{E_{1}}, R_{(a)_{3}}\right]$ for $a \in \mathfrak{S}$. $\widetilde{S}_{i}=\left\{(a)_{i}^{\prime} \in \mathscr{D} \mid a \in \mathfrak{S}\right\} \quad(1 \leq i \leq 3)$

Proposition 37. $\mathscr{D}=\mathscr{D}_{0} \oplus \widetilde{\Im}_{1} \oplus \mathfrak{S}_{2} \oplus \widetilde{J}_{3}, \operatorname{dim} \mathscr{D}=52$.

Proof. Let $D$ be any derivation of $\mathscr{J}$. As $E_{i} \circ E_{i}=E_{i}$, we get $2 E_{i} \circ\left(D E_{i}\right)=D E_{i}$ for $1 \leq i \leq 3$ and hence there exist $a, b, c, d, e, f, g \in \mathfrak{C}$ satisfying $D E_{1}=(a)_{3}-(b)_{2}, D E_{2}=(c)_{1}-(d)_{3}$ and $D E_{3}=(f)_{2}-(g)_{3}$. On the other hand, we get $E_{1} \circ\left(D E_{2}\right)+\left(D E_{1}\right) \circ E_{2}=0$ from $E_{1} \circ E_{2}=0$. This implies $(a)_{3}=(d)_{3}$. Thus we get $D E_{1}=(a)_{3}-(b)_{2}, D E_{2}=-(a)_{3}+(c)_{1}$ and $D E_{3}=(b)_{2}-(c)_{1}$. Put $T=4(a)_{3}^{\prime}-4(b)_{2}^{\prime}+4(c)_{1}^{\prime}$. Then $T$ is a derivation satisfying $T E_{1}=(a)_{3}-(b)_{2}, T E_{2}=-(a)_{3}+(c)_{1}, T E_{3}=(b)_{2}-(c)_{1}$ and hence $(D-T) E_{i}=0 \quad(1 \leq i \leq 3)$, i.e., $D-T \in \mathscr{D}_{0}$. This implies that $\mathscr{D}=\mathscr{D}_{0} \oplus \widetilde{\mathfrak{S}}_{1} \oplus \widetilde{\mathfrak{S}}_{2} \oplus \widetilde{\mathfrak{S}}_{3}$ and $\operatorname{dim} \mathscr{D}=52$ since $\operatorname{dim} \mathscr{D}_{0}=28, \operatorname{dim} \widetilde{\mathfrak{S}}_{i}=8$ $(1 \leq i \leq 3)$.

Q.E.D.

EXAMPLE 38. We shall study the derivation Lie algebra $\mathfrak{g}=\mathscr{D}$ of the exceptional simple Jordan algebra $\mathscr{J}$. Let $D$ be an element of $\mathscr{D}_{0}$ of the form in (1.12). Then $\operatorname{ad}(D) \cdot(a)_{1}^{\prime}=\left[D,\left[R_{E_{2}}, R_{(a)_{1}}\right]\right]=\left[R_{E_{2}},\left[D, R_{(a)_{1}}\right]\right]$ $+\left[\left[D, R_{E_{2}}\right], R_{(a)_{1}}\right]=\left[R_{E_{2}}, R_{D(a)_{1}}\right]+\left[R_{D E_{2}}, R_{(a)_{1}}\right]=\left[R_{E_{2}}, R_{(U a)_{1}}\right]=(U a)_{1}^{\prime}$. Similarly we have $\operatorname{ad}(D) \cdot(a)_{2}^{\prime}=\left(U^{\prime} a\right)_{2}^{\prime}, \operatorname{ad}(D) \cdot(a)_{3}^{\prime}=\left(U^{\prime \prime} a\right)_{3}^{\prime}$. Since $U^{\prime}, U^{\prime \prime}$ are two inequivalent half-spin representations of $U$ in $\mathfrak{p}(8, C)$, the adjoint representation of $\mathrm{g}$ induces a representation of $\mathscr{D}_{0} \cong \mathfrak{v}(8, C)$ on $\mathrm{g}$ which is the sum of the adjoint representation $\Lambda_{2}$ on $\mathscr{D}_{0}$, the standard representation $\Lambda_{1}$ on $\widetilde{S}_{1}$, and two inequivalent half-spin representations $\Lambda_{3}, \Lambda_{4}$ on $\widetilde{S}_{2}, \widetilde{\mathfrak{V}}_{3}$ (see Example 28). Hence a Cartan subalgebra $\mathfrak{h}$ of $\mathscr{D}_{0} \cong \mathfrak{o}(8, C)$ is a Cartan subalgebra of $g$ and the root system $\Delta$ is the sum of the weights of $\Lambda_{2}, \Lambda_{1}, \Lambda_{3}, \Lambda_{4}$, i.e., $\Delta=\left\{ \pm \lambda_{i} \pm \lambda_{j}, i<j=1,2,3,4 ; \pm \lambda_{i}, \pm \Lambda_{i}^{\prime}\right.$, $\pm \Lambda_{i}^{*}$, $i=1,2,3,4\}$ where $\Lambda_{i}^{\prime}, \Lambda_{i}^{*}$ are defined as in Example 28. Put $\alpha_{1}=\lambda_{2}-\lambda_{3}$, $\alpha_{2}=\lambda_{3}-\lambda_{4}, \alpha_{3}=\lambda_{4}, \alpha_{4}=\frac{1}{2}\left(\lambda_{1}-\lambda_{2}-\lambda_{3}-\lambda_{4}\right)$. Then, $\Pi=\left\{\alpha_{1}, \alpha_{2}, \alpha_{3}, \alpha_{4}\right\}$ is a fundamental root system, and under the lexicographical order of $\mathfrak{h}_{0}^{*}$ such that $\alpha_{i}(1 \leq i \leq 4)$ are simple roots, $\Delta_{+}=\left\{\alpha_{1}, \alpha_{2}, \alpha_{3}, \alpha_{4}, \alpha_{1}+\alpha_{2}, \alpha_{2}+\alpha_{3}, \alpha_{3}+\right.$ $\alpha_{4}, \alpha_{1}+\alpha_{2}+\alpha_{3}, \alpha_{2}+\alpha_{3}+\alpha_{4}, \alpha_{2}+2 \alpha_{3}, \alpha_{1}+\alpha_{2}+\alpha_{3}+\alpha_{4}, \alpha_{1}+\alpha_{2}+2 \alpha_{3}, \alpha_{2}+$ $2 \alpha_{3}+\alpha_{4}, \alpha_{1}+\alpha_{2}+2 \alpha_{3}+\alpha_{4}, \alpha_{1}+2 \alpha_{2}+2 \alpha_{3}, \alpha_{2}+2 \alpha_{3}+2 \alpha_{4}, \alpha_{1}+\alpha_{2}+2 \alpha_{3}+$ $2 \alpha_{4}, \alpha_{1}+2 \alpha_{2}+2 \alpha_{3}+\alpha_{4}, \alpha_{1}+2 \alpha_{2}+2 \alpha_{3}+2 \alpha_{4}, \alpha_{1}+2 \alpha_{2}+3 \alpha_{3}+\alpha_{4}, \alpha_{1}+2 \alpha_{2}$ $\left.+3 \alpha_{3}+2 \alpha_{4}, \alpha_{1}+2 \alpha_{2}+4 \alpha_{3}+2 \alpha_{4}, \alpha_{1}+3 \alpha_{2}+4 \alpha_{3}+2 \alpha_{4}, 2 \alpha_{1}+3 \alpha_{2}+4 \alpha_{3}+2 \alpha_{4}\right\}$ and $\rho=\sum_{\alpha \in \Lambda_{+}} \alpha=8 \alpha_{1}+15 \alpha_{2}+21 \alpha_{3}+11 \alpha_{4}$. Let $B$ be the Killing form of g. Then for $D=D_{0}+(a)_{1}^{\prime}+(b)_{2}^{\prime}+(c)_{3}^{\prime} \in \mathfrak{g}, D_{0} \in \mathscr{D}_{0}, B(D, D)=\frac{3}{2} B^{\prime}\left(D_{0}, D_{0}\right)$ $-\frac{9}{2}(a \bar{a}+b \bar{b}+c \bar{c})$ where $B^{\prime}$ is the Killing form of $\mathscr{D}_{0} \cong \mathfrak{v}(8, C)$ (see p. 111 
[5]). As $B$ is non-degenerate, $\mathfrak{g}$ is semi-simple. Since $B(H, H)=18 \sum_{i=1}^{4} \lambda_{i}^{2}$ for $H=H\left(\lambda_{1}, \lambda_{2}, \lambda_{3}, \lambda_{4}\right) \in \mathfrak{h}$, we get

$$
H_{\Sigma m_{i} \lambda_{i}}=H\left(\frac{m_{1}}{18}, \frac{m_{2}}{18}, \frac{m_{3}}{18}, \frac{m_{4}}{18}\right),
$$

$\left(\sum m_{i} \lambda_{i}, \sum n_{j} \lambda_{j}\right)=\frac{1}{18} \sum_{i=1}^{4} m_{i} n_{i}$ and in particular $\left(\alpha_{1}, \alpha_{1}\right)=\left(\alpha_{2}, \alpha_{2}\right)=\frac{1}{9}$, $\left(\alpha_{3}, \alpha_{3}\right)=\left(\alpha_{4}, \alpha_{4}\right)=\frac{1}{18}, \quad\left(\alpha_{1}, \alpha_{2}\right)=\left(\alpha_{2}, \alpha_{3}\right)=-\frac{1}{18}, \quad\left(\alpha_{3}, \alpha_{4}\right)=-\frac{1}{36}, \quad\left(\alpha_{1}, \alpha_{3}\right)=$ $\left(\alpha_{1}, \alpha_{4}\right)=\left(\alpha_{2}, \alpha_{4}\right)=0$. This shows that the Dynkin diagram of $\mathfrak{g}$ is $\stackrel{\alpha_{1}}{\circ} \quad \alpha_{2} \Longrightarrow \stackrel{\alpha_{3}}{\circ} \quad \alpha_{4}^{\alpha_{4}}$ ○ and $\mathrm{g}=\mathscr{D}$ is the simple Lie algebra of type $F_{4}$. Put $\Lambda_{1}=18\left(2 \alpha_{1}+3 \alpha_{2}+4 \alpha_{3}+2 \alpha_{4}\right), \quad \Lambda_{2}=18\left(3 \alpha_{1}+6 \alpha_{2}+8 \alpha_{3}+4 \alpha_{4}\right), \quad \Lambda_{3}=$ $18\left(2 \alpha_{1}+4 \alpha_{2}+6 \alpha_{3}+3 \alpha_{4}\right)$, and $\Lambda_{4}=18\left(\alpha_{1}+2 \alpha_{2}+3 \alpha_{3}+2 \alpha_{4}\right)$. Then since $\frac{2\left(\Lambda_{i}, \alpha_{j}\right)}{\left(\alpha_{j}, \alpha_{j}\right)}=\delta_{i j}$ for $1 \leq i, j \leq 4, \Lambda_{1}, \Lambda_{2}, \Lambda_{3}, \Lambda_{4}$ are the fundamental dominant weights. Let $\Lambda=\sum_{i=1}^{4} m_{i} \Lambda_{i}\left(m_{i} \geqq 0\right.$, integer $)$ be any dominant integral form. Then the Weyl's dimension formula says that $d(\Lambda)=\prod_{\alpha \in \Delta_{+}} \frac{(\Lambda+\rho, \alpha)}{(\rho, \alpha)}$ $=\frac{1}{2^{15} \cdot 3^{7} \cdot 5^{4} \cdot 7^{2} \cdot 11}\left(m_{1}+1\right)\left(m_{2}+1\right)\left(m_{3}+1\right)\left(m_{4}+1\right)\left(m_{1}+m_{2}+2\right)\left(2 m_{2}+m_{3}\right.$ $+3)\left(m_{3}+m_{4}+2\right)\left(m_{2}+m_{3}+2\right)\left(2 m_{1}+2 m_{2}+m_{3}+5\right)\left(2 m_{2}+m_{3}+m_{4}+4\right)$ $\left(2 m_{1}+2 m_{2}+m_{3}+m_{4}+6\right)\left(m_{1}+m_{2}+m_{3}+3\right)\left(2 m_{2}+2 m_{3}+m_{4}+5\right)\left(m_{2}+\right.$ $\left.m_{3}+m_{4}+3\right)\left(m_{1}+2 m_{2}+m_{3}+4\right)\left(2 m_{1}+2 m_{2}+2 m_{3}+m_{4}+7\right)\left(m_{1}+m_{2}+\right.$ $\left.m_{3}+m_{4}+4\right)\left(2 m_{1}+4 m_{2}+2 m_{3}+m_{4}+9\right)\left(m_{1}+2 m_{2}+m_{3}+m_{4}+5\right)\left(2 m_{1}+\right.$ $\left.4 m_{2}+3 m_{3}+m_{4}+10\right)\left(2 m_{1}+4 m_{2}+3 m_{3}+2 m_{4}+11\right)\left(m_{1}+2 m_{2}+2 m_{3}+m_{4}\right.$ $+6)\left(m_{1}+3 m_{2}+2 m_{3}+m_{4}+7\right)\left(2 m_{1}+3 m_{2}+2 m_{3}+m_{4}+8\right)$. For example, $d\left(\Lambda_{4}\right)=26, d\left(\Lambda_{1}\right)=52, d\left(\Lambda_{3}\right)=273, d\left(2 \Lambda_{4}\right)=324, d\left(2 \Lambda_{1}\right)=d\left(\Lambda_{1}+\Lambda_{4}\right)=1053$, $d\left(\Lambda_{2}\right)=1274, d\left(3 \Lambda_{4}\right)=2652, d\left(2 \Lambda_{3}\right)=19448, d\left(2 \Lambda_{2}\right)=226746$, etc. Let $\mathscr{J}_{0}=$ $\{X \in \mathscr{J} \mid \operatorname{Tr} X=0\}$ be a 26-dimensional subspace of $\mathscr{J}$ over $C$. Then, $\mathscr{J}_{0}$ is an invariant subspace of the derivation algebra $F_{4}=\mathscr{D}$ of $\mathscr{J}$, i.e., $\mathscr{J}_{0}$ is an irreducible representation space of $\Lambda_{4}$. Obviously, $\Lambda_{1}$ is the adjoint representation of $F_{4}$.

EXAMPLE 39. Let $g$ be the Lie algebra spanned by the derivations $\left(=F_{4}\right)$ of $\mathscr{J}$ and the right multiplications of elements $Y$ of trace 0 . If $X$ and $Y$ are in $\mathscr{J}$, then $\left[R_{X}, R_{Y}\right]$ is in $\mathscr{D}\left(=F_{4}\right) ;$ moreover, if $D \in \mathscr{D}$, then $\left[D, R_{Y}\right]=R_{D Y}$ and $\operatorname{Tr} D Y=0$. It follows that $\mathrm{g}=\mathscr{D}+\left\{R_{Y}\right\}, \operatorname{Tr} Y=0$. Since $D(1)=0$ for every derivation $D$ of $\mathscr{J}, D+R_{Y}=0$ implies $Y=0$, $D=0$; thus $\mathfrak{g}$ is of dimension 78 , and the adjoint representation of $\mathfrak{g}$ 
induces a representation of $\mathscr{D}\left(=F_{4}\right)$ which is the sum of the adjoint representation $\Lambda_{1}$ and of the representation $\Lambda_{4}$ whose space is the set $\mathscr{R}$ of right multiplications of elements in $\mathscr{J}_{0}$ Since $\mathscr{D}$ and $\mathscr{R}$ yield irreducible representation spaces of $\mathscr{D}$ of distinct dimensions, the only possible ideals are $\{0\}, \mathscr{D}, \mathscr{R}$ and $\mathscr{D}+\mathscr{R}=g$; but $\mathscr{D}$ and $\mathscr{R}$ are obviously not ideals, which proves that $g$ is simple (these are quoted from [4]). Let $B$ be the Killing form of $\mathfrak{g}$. Then

$$
B\left(R_{a}+D, R_{a}+D\right)=12 \operatorname{Tr} a \circ a+\frac{4}{3} B^{\prime}(D, D)
$$

where $B^{\prime}$ is the Killing form of $F_{4}=\mathscr{D}$. Let $\mathfrak{h}^{\prime}$ be a Cartan subalgebra of $F_{4}=\mathscr{D}$ and let $H_{1}, H_{2}, H_{3}, H_{4}$ be its basis satisfying $H\left(\lambda_{1}, \lambda_{2}, \lambda_{3}, \lambda_{4}\right)=\sum_{i=1}^{4} \lambda_{i} H_{i}$ for any $H\left(\lambda_{1}, \cdots, \lambda_{4}\right) \in \mathfrak{G}^{\prime}$. Set $H_{5}=R_{E_{1}}, H_{6}=R_{E_{2}}, H_{7}=R_{E_{3}}$ where $E_{i}$ are as in Definition 34. Then $\mathfrak{h}=\left\{\sum_{i=1}^{7} \lambda_{i} H_{i} \mid \lambda_{5}+\lambda_{6}+\lambda_{7}=0\right\}$ is a Cartan subalgebra of g. The root system $\Delta$ of $\mathrm{g}$ w.r.t. $\mathfrak{h}$ is given by $\Delta=\left\{ \pm \lambda_{i} \pm \lambda_{j}, i<j=\right.$ $\left.1,2,3,4 ; \pm \lambda_{i} \pm \frac{1}{2}\left(\lambda_{5}-\lambda_{7}\right) ; \pm \Lambda_{i}^{\prime} \pm \frac{1}{2}\left(\lambda_{5}-\lambda_{6}\right) ; \pm \Lambda_{i}^{*} \pm \frac{1}{2}\left(\lambda_{6}-\lambda_{7}\right), i=1,2,3,4\right\}$ where $\Lambda_{i}^{\prime}, \Lambda_{i}^{*}$ are defined as in Example 28. Put $\alpha_{1}=-\Lambda_{1}^{\prime}+\frac{1}{2}\left(\lambda_{5}-\lambda_{6}\right), \alpha_{2}$ $=\lambda_{4}-\frac{1}{2}\left(\lambda_{5}-\lambda_{7}\right), \alpha_{3}=\lambda_{3}-\lambda_{4}, \alpha_{4}=\lambda_{4}+\frac{1}{2}\left(\lambda_{5}-\lambda_{7}\right), \alpha_{5}=-\Lambda_{1}^{\prime}-\frac{1}{2}\left(\lambda_{5}-\lambda_{6}\right), \alpha_{6}$ $=\lambda_{2}-\lambda_{3}$. Then $\Pi=\left\{\alpha_{1}, \cdots, \alpha_{6}\right\}$ is a fundamental root system. Since

$$
B\left(\sum \lambda_{i} H_{i}, \sum \lambda_{i}^{\prime} H_{i}\right)=24 \sum_{i=1}^{4} \lambda_{i} \lambda_{i}^{\prime}+12 \sum_{i=5}^{7} \lambda_{i} \lambda_{i}^{\prime},
$$

we obtain that $\left(\sum m_{i} \lambda_{i}, \sum n_{j} \lambda_{j}\right)=\frac{1}{24} \sum_{i=1}^{4} m_{i} n_{i}+\frac{1}{12} \sum_{i=5}^{7} m_{i} n_{i}$, in particular $\left(\alpha_{i}, \alpha_{i}\right)=\frac{1}{12}$ for $1 \leq i \leq 6,\left(\alpha_{i}, \alpha_{i+1}\right)=-\frac{1}{24}$ for $1 \leq i \leq 4$, and $\left(\alpha_{i}, \alpha_{j}\right)=0$ for $|i-j| \geqq 2$ except for $\left(\alpha_{3}, \alpha_{6}\right)=-\frac{1}{24}$. This shows that the Dynkin diagram

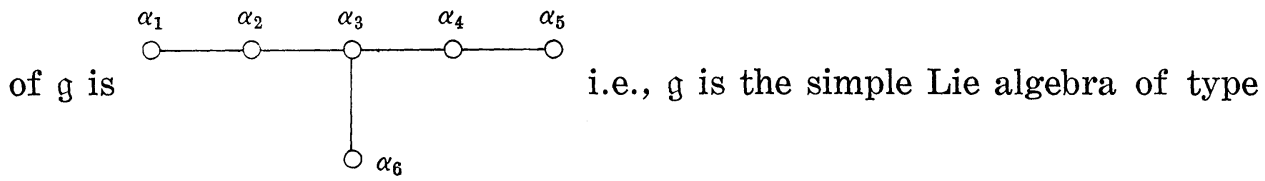

$E_{6}$. Under the lexicographical order in $\mathfrak{h}_{0}^{*}$ such that $\Pi=\left\{\alpha_{1}, \cdots, \alpha_{6}\right\}$ are simple roots, all positive roots $\Delta_{+}$is given by $\Delta_{+}=\left\{\alpha_{1}, \alpha_{2}, \alpha_{3}, \alpha_{4}, \alpha_{5}, \alpha_{6}\right.$; $\alpha_{1}+\alpha_{2}, \alpha_{2}+\alpha_{3}, \alpha_{3}+\alpha_{4}, \alpha_{4}+\alpha_{5}, \alpha_{3}+\alpha_{6} ; \alpha_{1}+\alpha_{2}+\alpha_{3}, \alpha_{2}+\alpha_{3}+\alpha_{4}, \alpha_{3}+\alpha_{4}+$ $\alpha_{5}, \alpha_{3}+\alpha_{4}+\alpha_{6}, \alpha_{2}+\alpha_{3}+\alpha_{6} ; \alpha_{1}+\alpha_{2}+\alpha_{3}+\alpha_{4}, \alpha_{2}+\alpha_{3}+\alpha_{4}+\alpha_{5}, \alpha_{3}+\alpha_{4}+$ $\alpha_{5}+\alpha_{6}, \alpha_{1}+\alpha_{2}+\alpha_{3}+\alpha_{6}, \alpha_{2}+\alpha_{3}+\alpha_{4}+\alpha_{6}, \alpha_{1}+\alpha_{2}+\alpha_{3}+\alpha_{4}+\alpha_{5}, \alpha_{2}+\alpha_{3}$ $+\alpha_{4}+\alpha_{5}+\alpha_{6}, \alpha_{2}+2 \alpha_{3}+\alpha_{4}+\alpha_{6}, \alpha_{1}+\alpha_{2}+\alpha_{3}+\alpha_{4}+\alpha_{6} ; \alpha_{1}+\alpha_{2}+\alpha_{3}+\alpha_{4}$ $+\alpha_{5}+\alpha_{6}, \alpha_{1}+\alpha_{2}+2 \alpha_{3}+\alpha_{4}+\alpha_{6}, \alpha_{2}+2 \alpha_{3}+\alpha_{4}+\alpha_{5}+\alpha_{6} ; \alpha_{1}+\alpha_{2}+2 \alpha_{3}+\alpha_{4}$ $+\alpha_{5}+\alpha_{6}, \alpha_{1}+2 \alpha_{2}+2 \alpha_{3}+\alpha_{4}+\alpha_{6}, \alpha_{2}+2 \alpha_{3}+2 \alpha_{4}+\alpha_{5}+\alpha_{6} ; \alpha_{1}+\alpha_{2}+2 \alpha_{3}$ $+2 \alpha_{4}+\alpha_{5}+\alpha_{6}, \alpha_{1}+2 \alpha_{2}+2 \alpha_{3}+\alpha_{4}+\alpha_{5}+\alpha_{6} ; \alpha_{1}+2 \alpha_{2}+2 \alpha_{3}+2 \alpha_{4}+\alpha_{5}+$ 
$\left.\alpha_{6} ; \alpha_{1}+2 \alpha_{2}+3 \alpha_{3}+2 \alpha_{4}+\alpha_{5}+\alpha_{6} ; \alpha_{1}+2 \alpha_{2}+3 \alpha_{3}+2 \alpha_{4}+\alpha_{5}+2 \alpha_{6}\right\}$. Hence we obtain that $\rho=\frac{1}{2} \sum_{\alpha \in \Delta_{+}} \alpha=8 \alpha_{1}+15 \alpha_{2}+21 \alpha_{3}+15 \alpha_{4}+8 \alpha_{5}+11 \alpha_{6}$, and $\left(\rho, \alpha_{i}\right)=\frac{1}{24}$ for $1 \leq i \leq 6$. Let $\Lambda_{1}, \cdots, \Lambda_{6}$ be the fundamental weights, i.e., $\frac{2\left(\Lambda_{i}, \alpha_{j}\right)}{\left(\alpha_{j}, \alpha_{j}\right)}=\delta_{i j}$ for $i, j,=1, \cdots, 6$. Let $\Lambda=\sum_{i=1}^{6} m_{i} \Lambda_{i}\left(m_{i} \geqq 0\right.$, integer) be any dominant integral form of $\mathrm{g}=E_{6}$. Since $\left(\Lambda+\rho, \alpha_{i}\right)=\frac{1}{2} m_{i}\left(\alpha_{i}, \alpha_{i}\right)$ $+\frac{1}{24}=\frac{1}{24}\left(m_{i}+1\right)$ for $1 \leq i \leq 6$, the Weyl's dimension formula says that

$$
d(\Lambda)=\prod_{\alpha \in \Delta_{+}} \frac{(\Lambda+\rho, \alpha)}{(\rho, \alpha)}=\prod_{\sum n_{i} \alpha_{i} \in \Delta_{+}} \frac{\left(\sum_{i=1}^{6} n_{i}\left(m_{i}+1\right)\right)}{\left(\sum_{i=1}^{6} n_{i}\right)} .
$$

For example, $d\left(\Lambda_{1}\right)=d\left(\Lambda_{5}\right)=27, d\left(\Lambda_{8}\right)=78, d\left(2 \Lambda_{1}\right)=d\left(\Lambda_{2}\right)=d\left(\Lambda_{4}\right)=351$, $d\left(\Lambda_{3}\right)=2925, d\left(2 \Lambda_{6}\right)=2430$, etc. Here $\Lambda_{1}$ and $\Lambda_{5}$ are contragredient of each other and so do $\Lambda_{2}$ and $\Lambda_{4}$. Let $d \rho_{i}$ be the fundamental irreducible representations of $E_{6}(1 \leq i \leq 6)$. Then there exists an outer automorphism $\sigma$ of $E_{6}$ such that $d \rho_{5}$ and $d \rho_{1} \circ \sigma$ are equivalent. The representation space of $\Lambda_{1}$ (and $\Lambda_{5}$ ) is the exceptional simple Jordan algebra $\mathscr{J}$. $\Lambda_{6}$ is the adjoint representation.

Let $N(X)=\operatorname{det} X=\xi_{1} \xi_{2} \xi_{3}+\operatorname{tr} x_{1} x_{2} x_{3}-\xi_{1} x_{1} \bar{x}_{1}-\xi_{2} x_{2} \bar{x}_{2}-\xi_{3} x_{3} \bar{x}_{3}$ be the determinant of $X$ of the form (1.9) in $\mathscr{J}$. Then $E_{6}$ leaves $N(X)$ invariant. Let $N(X, Y, Z)$ be the trilinear form obtained by polarizing $N(X)$. Then the Lie algebra $E_{6}$ can be characterized as the set of linear transformations $L$ of $\mathscr{J}$ such that $N(L X, Y, Z)+N(X, L Y, Z)+N(X, Y, L Z)=0$.

Example 40 . We shall construct the Lie algebra of type $E_{7}$. Let $\overline{\mathscr{E}}_{6}(\mathscr{J})$ be the Lie algebra spanned by the derivations $\mathscr{D}\left(=F_{4}\right)$ of $\mathscr{J}$ and and the right multiplications of elements $Y$, but not necessarily of trace 0 , i.e., $\overline{\mathscr{E}}_{6}(\mathscr{J})=E_{6} \oplus C R_{I}$ where $I$ is the unit matrix in $\mathscr{J}$. Let $\bar{J}$ be a vector space isomorphic to $\mathscr{J}$ under the mapping $X \mapsto \bar{X}$. We shall define the structure of a Lie algebra in a vector space $g=\mathscr{J} \oplus \bar{J} \oplus \overline{\mathscr{E}}_{6}(\mathscr{F})$ of dimension 133 over $C$ such that $\overline{\mathscr{E}}_{6}(\mathscr{J})$ is a subalgebra and

$$
\begin{aligned}
{[X, Y]=0 } & =[\bar{X}, \bar{Y}] \quad \text { for } X, Y \in \mathscr{J} \\
{[X, \bar{Y}] } & =2 R_{X \circ Y}+2\left[R_{Y}, R_{X}\right] \\
{[L, X] } & =L X \quad \text { for } L \in \overline{\mathscr{E}}_{6}(\mathscr{J}), X \in \mathscr{J} \\
{[L, \bar{X}] } & =\bar{L} X \quad \text { where } \bar{L}=-R_{Y}+D \text { if } \\
L & =R_{Y}+D, D \in \mathscr{D}\left(=F_{4}\right) .
\end{aligned}
$$

The Killing form $B$ of $g$ is given by $B\left(A, A^{\prime}\right)=2 B^{\prime}\left(D, D^{\prime}\right)+18 T\left(Z, Z^{\prime}\right)$ 
$-36\left(T\left(X, Y^{\prime}\right)+T\left(Y, X^{\prime}\right)\right)$ for $A=\left(X, \bar{Y}, R_{Z}+D\right), A^{\prime}=\left(X^{\prime}, \bar{Y}^{\prime}, R_{Z^{\prime}}+\right.$ $\left.D^{\prime}\right) \in \mathfrak{g}$ where $B^{\prime}$ is the Killing form of $F_{4}$ and $T(X, Y)=\operatorname{Tr} X \circ Y$ for $X, Y \in \mathscr{J}$. Since $B$ is non-degenerate, the Lie algebra $g$ is semi-simple. Let $\mathfrak{h}=\left\{\sum_{i=1}^{7} \lambda_{i} H_{i} \mid \lambda_{1}, \cdots, \lambda_{7} \in C\right\}$ be the subalgebra of $\overrightarrow{\mathscr{E}}_{6}(\mathscr{J})$ where $\left\{H_{1}\right.$, $\left.\cdots, H_{4}\right\}$ is a basis of a Cartan subalgebra of $F_{4}$ and $H_{5}=R_{E_{1}}, H_{6}=R_{E_{2}}$, $H_{7}=R_{E_{3}}$ (see Example 39), but here we don't assume that $\lambda_{5}+\lambda_{6}+\lambda_{7}=0$. Then $\mathfrak{h}$ is a Cartan subalgebra of $\mathfrak{g}$, and the root system $\Delta$ of $\mathfrak{g}$ w.r.t. $\mathfrak{h}$ is given by $\Delta=\left\{ \pm \lambda_{i} \pm \lambda_{j}, i<j=1,2,3,4, \pm \lambda_{i} \pm \frac{1}{2}\left(\lambda_{5}-\lambda_{7}\right), \pm \Lambda_{i}^{\prime} \pm \frac{1}{2}\left(\lambda_{5}-\lambda_{6}\right)\right.$, $\pm \Lambda_{i}^{*} \pm \frac{1}{2}\left(\lambda_{6}-\lambda_{7}\right), \quad \pm \lambda_{i} \pm \frac{1}{2}\left(\lambda_{5}+\lambda_{7}\right), \quad \pm \Lambda_{i}^{\prime} \pm \frac{1}{2}\left(\lambda_{5}+\lambda_{6}\right), \quad \pm \Lambda_{i}^{*} \pm \frac{1}{2}\left(\lambda_{6}+\lambda_{7}\right), \quad i=$ $\left.1,2,3,4, \pm \lambda_{5}, \pm \lambda_{6}, \pm \lambda_{7}\right\}$ where $\Lambda_{i}^{\prime}, \Lambda_{i}^{*}$ are defined as in Example 28. Put $\alpha_{1}=\lambda_{2}-\lambda_{3}, \alpha_{2}=\lambda_{3}-\lambda_{4}, \alpha_{3}=\lambda_{4}-\frac{1}{2}\left(\lambda_{5}+\lambda_{7}\right), \alpha_{4}=\lambda_{5}, \alpha_{5}=\frac{1}{2}\left(\lambda_{1}-\lambda_{2}-\lambda_{3}-\lambda_{4}\right.$ $\left.-\lambda_{5}-\lambda_{6}\right), \alpha_{6}=\lambda_{6}, \alpha_{7}=\lambda_{7}$. Then $\Pi=\left\{\alpha_{1}, \cdots, \alpha_{7}\right\}$ is a fundamental root system of g. Since $B\left(\sum \lambda_{i} H_{i}, \sum \lambda_{i}^{\prime} H_{i}\right)=36 \sum_{i=1}^{4} \lambda_{i} \lambda_{i}^{\prime}+18 \sum_{i=5}^{7} \lambda_{i} \lambda_{i}^{\prime}$, we obtain the inner product $\left(\sum m_{i} \lambda_{i}, \sum n_{j} \lambda_{j}\right)=\frac{1}{36} \sum_{i=1}^{4} m_{i} n_{i}+\frac{1}{18} \sum_{i=5}^{7} m_{i} n_{i}$. Hence $\left(\alpha_{i}, \alpha_{i}\right)=\frac{1}{18}$ for $1 \leq i \leq 7,\left(\alpha_{3}, \alpha_{7}\right)=\left(\alpha_{i}, \alpha_{i+1}\right)=-\frac{1}{36}(1 \leq i \leq 5)$, and $\left(\alpha_{i}, \alpha_{j}\right)=0$ if $|i-j| \geqq 2$ except $\left(\alpha_{3}, \alpha_{7}\right)=\left(\alpha_{7}, \alpha_{3}\right)$. This shows that the

Dynkin diagram of $g$ is

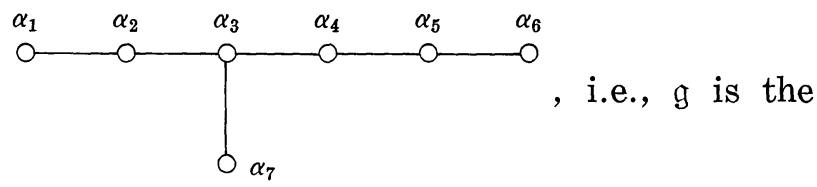

simple Lie algebra of type $E_{7}$. Since we have $\Delta$ and $\Pi$, we can easily determine 63-positive roots $\Delta_{+}$and we get $2 \rho=\sum_{\alpha \in \Delta_{+}} \alpha=34 \alpha_{1}+66 \alpha_{2}+$ $96 \alpha_{3}+75 \alpha_{4}+52 \alpha_{5}+27 \alpha_{6}+49 \alpha_{7}$ and $\left(\rho, \alpha_{i}\right)=\frac{1}{36}(1 \leq i \leq 7)$. Let $\Lambda_{1}, \cdots, \Lambda_{7}$ be the fundamental weights of $g=E_{7}$, i.e., $\frac{\left(\Delta_{i}, \alpha_{j}\right)}{\left(\alpha_{j}, \alpha_{j}\right)}=\delta_{i j} i, j=1, \cdots, 7$, and let $\Lambda=\sum_{i=1}^{\tau} m_{i} \Lambda_{i}\left(m_{i} \geqq 0\right.$, integer $)$ be any dominant integral form. Then $\left(\Lambda+\rho, \alpha_{i}\right)=\frac{m_{i}}{2}\left(\alpha_{i}, \alpha_{i}\right)+\left(\rho, \alpha_{i}\right)=\frac{1}{36}\left(m_{i}+1\right)$, and hence $(\Lambda+\rho$, $\left.\sum n_{i} \alpha_{i}\right)+\frac{1}{36} \sum_{i=1}^{7} n_{i}\left(m_{i}+1\right)$. The Weyl's dimension formula says that

$$
d(\Lambda)=\prod_{\alpha \in A_{+}} \frac{(\Lambda+\rho, \alpha)}{(\rho, \alpha)}=\prod_{\sum n_{i} \alpha_{i} \in \Delta_{+}} \frac{\sum_{i=1}^{7} n_{i}\left(m_{i}+1\right)}{\left(\sum_{i=1}^{7} n_{i}\right)} .
$$

For example, $d\left(\Lambda_{1}\right)=133, d\left(\Lambda_{2}\right)=8645, d\left(\Lambda_{3}\right)=365750, d\left(\Lambda_{4}\right)=27664$, $d\left(\Lambda_{5}\right)=1539, d\left(\Lambda_{6}\right)=56, d\left(\Lambda_{7}\right)=912, d\left(2 \Lambda_{1}\right)=7371, d\left(2 \Lambda_{6}\right)=1463, d\left(\Lambda_{1}+\right.$ $\left.\Lambda_{6}\right)=3920$, etc. Obviously $\Lambda_{1}$ is the adjoint representation of $\mathfrak{g}=E_{7}$. We shall construct the representation $\Lambda_{6}$ of degree 56. For this purpose, we shall define the Freudenthal product $\times$ in $\mathscr{J}$ by a $\times b=a \circ b-$ $\frac{1}{2} \operatorname{Tr}(a) b-\frac{1}{2} \operatorname{Tr}(b) a+\frac{1}{2}[\operatorname{Tr}(a) \cdot \operatorname{Tr}(b)-\operatorname{Tr} a \circ b] \cdot 1$ for $a, b \in \mathscr{J}$. Let $A^{*}$ 
denote the adjoint of a linear transformation $A$ in $\mathscr{J}$ relative to the trace form $T(a, b)=\operatorname{Tr} a \circ b$, i.e., $T(A a, b)=T\left(a, A^{*} b\right)$. Then we know that $R_{a}$ is self-adjoint.

We now define $\mathfrak{M}=C \oplus C \oplus \mathscr{J} \oplus \mathscr{J}$ a vector space direct sum of two copies of $C$ and two copies of $\mathscr{J}$, so $\operatorname{dim} \mathfrak{M}=56$. We write the elements of $\mathfrak{M}$ as $X=(\xi, \eta, x, y)$ where $\xi, \eta \in C, x, y \in \mathscr{J}$, and we define an action of $\mathrm{g}=\mathscr{J} \oplus \bar{J} \oplus \overline{\mathscr{E}}_{6}(\mathscr{J})$ by the following formulas :

$$
\begin{aligned}
{[a, X] } & =(T(a, y), 0, \eta a, 2 a \times x) \quad \text { for } a \in \mathscr{J} \\
{[\bar{a}, X] } & =(0,-T(a, x),-2 a \times y,-\xi a) \quad \text { for } \bar{a} \in \bar{J} \\
{\left[2 R_{I}, X\right] } & =(3 \xi,-3 \eta,-x, y) \\
{[L, X] } & =\left(0,0, L x,-L^{*} y\right) \quad \text { for } L \in \mathscr{E}_{6}(\mathscr{J}) .
\end{aligned}
$$

Here, $\mathscr{E}_{6}(\mathscr{J})$ is a subalgebra of $\overline{\mathscr{E}}_{6}(\mathscr{F})$ of elements of the form $R_{a}+D$, $\operatorname{Tr} a=0, D$ a derivation. Then the action of $\mathfrak{g}$ on $\mathfrak{M}$ thus defined gives an irreducible representation of $\mathfrak{g}$ on $\mathfrak{M}$ of the highest weight $\Lambda_{6}$. Define a non-degenerate skew bilinear form $\{$,$\} and a quartic form q$ on $\mathfrak{M}$ by

$$
\begin{gathered}
\left\{X_{1}, X_{2}\right\}=\xi_{1} \eta_{2}-\xi_{2} \eta_{1}+T\left(x_{1}, y_{2}\right)-T\left(x_{2}, y_{1}\right) \\
q(X)=T\left(x^{\sharp}, y^{\sharp}\right)-\xi N(x)-\eta N(y)-\frac{1}{4}(T(x, y)-\xi \eta)^{2}
\end{gathered}
$$

where $a^{\sharp}=a^{2}-\operatorname{Tr}(\alpha) \cdot a+\frac{1}{2}\left\{(\operatorname{Tr} \alpha)^{2}-\operatorname{Tr}\left(a^{2}\right)\right\} \cdot 1$, and $N(x)=\operatorname{det} x$ (see Example 39), $\quad T(x, y)=\operatorname{Tr} x \circ y, \quad X=(\xi, \eta, x, y), \quad X_{i}=\left(\xi_{i}, \eta_{i}, x_{i}, y_{i}\right)$ for $i=1,2$.

It can be shown that if $q\left(X_{1}, X_{2}, X_{3}, X_{4}\right)$ is the symmetric 4-linear form obtained by polarizing $q$, then the Lie of algebra linear transformations in $\mathfrak{M}$ corresponding to $\mathfrak{g}$ can be characterized as the set of linear transformations $A$ of $\mathfrak{M}$ such that $q\left(A X_{1}, X_{2}, X_{3}, X_{4}\right)+q\left(X_{1}, A X_{2}, X_{3}, X_{4}\right)+$ $q\left(X_{1}, X_{2}, A X_{3}, X_{4}\right)+q\left(X_{1}, X_{2}, X_{3}, A X_{4}\right)=0$. Also direct verification using (1.14) shows that $\left\{A X_{1}, X_{2}\right\}+\left\{X_{1}, A X_{2}\right\}=0$ for $A \in \mathfrak{g}$.

EXAMPLE 41 . We shall construct the Lie algebra of type $E_{8}$. Only the fact that the least dimensional representation of $E_{8}$ is the adjoint representation will be used later. For this purpose, we consider first the derivation algebra Der(ㄷ) of the Cayley algebra 5 (see Definition 31). Put $f_{1}=\frac{1+\sqrt{-1} e_{1}}{2}, f_{2}=\frac{1-\sqrt{-1} e_{1}}{2}=1-f_{1}$. Then $f_{i}^{2}=f_{i}$ $(i=1,2), f_{1} f_{2}=f_{2} f_{1}=0, f_{1}+f_{2}=1$, and $f_{1}=\bar{f}_{2}, f_{2}=\bar{f}_{1}$. Since $f_{i}\left(f_{i}\right.$ $=C f_{i}(i=1,2)$, we obtain the Peirce decomposition $\mathbb{E}=\left(f_{1}+f_{2}\right) \mathfrak{E}\left(f_{1}+\right.$ $\left.f_{2}\right)=C f_{1} \oplus C f_{2} \oplus f_{1} \Subset f_{2} \oplus f_{2} \Subset f_{1}$ of $\Subset$, where $f_{1} \Subset f_{2}$ and $f_{2} \Subset f_{1}$ are threedimensional. 
Let $\mathfrak{D}_{0}$ be the subalgebra of $\operatorname{Der}(\mathfrak{S})$ of derivations mapping $f_{1}$ (and hence $f_{2}$ ) into 0 . Such a $D$ maps the Peirce components $f_{i}\left(f_{j}\right.$ into themselves. The representations of $\mathfrak{D}_{0}$ in the space $f_{i} \Subset f_{j}(i \neq j)$ can be considered as the standard representation of $\mathscr{D}_{0} \cong \mathfrak{I l}(3, C)$ and its contragredient. Let $D$ be any derivation in $\mathfrak{C}^{5}$. We have $D f_{i}=f_{i}\left(D f_{i}\right)+$ $\left(D f_{i}\right) f_{i}$ since $f_{i}^{2}=f_{i}(i=1,2)$ and hence, together with $D f_{1}=-D f_{2}$, $D f_{1} \in f_{1} \Subset f_{2}+f_{2} \Subset f_{1}$ so that we have $D f_{1}=a_{12}-b_{21}, D f_{2}=-a_{12}+b_{21}$ where $a_{12} \in f_{1} \Subset f_{2}, b_{21} \in f_{2} \Subset f_{1}$. Put $D_{a, b}=(a b)_{R}-(a b)_{L}-3\left[a_{L} b_{R}\right]$ for $a, b \in \Subset$ where we write $a_{R}$ for $X \rightarrow X a$ and $a_{L}$ for $X \rightarrow a X$. Then $D_{a, b}$ is a derivation of $\Subset$ and $D_{f_{1}, a_{12}} \cdot f_{1}=a_{12}, D_{f_{2}, b_{21}} f_{2}=b_{21}$ for $a_{12} \in f_{1} \Subset f_{2}, b_{21} \in f_{2} \Subset f_{1}$. Thus we obtain that $E=D-D_{f_{1}, a_{12}}-D_{f_{2}, b_{21}} \in \mathscr{D}_{0}$, i.e., $D=E+D_{f_{1}, a_{12}}+D_{f_{2}, b_{21}}$. Since this expression is unique, we have $\operatorname{Der}(\mathfrak{S})=\mathscr{D}_{0} \oplus \mathfrak{S}_{1} \oplus \mathfrak{S}_{2}, \widetilde{\mathfrak{S}}_{1}=$ $\left\{D_{f_{2}, b_{21}} \mid b_{21} \in f_{2}\left(f_{1}\right\}, \widetilde{J}_{2}=\left\{D_{f_{1}, a_{12}} \mid a_{12} \in f_{1} \mathfrak{E} f_{2}\right\}\right.$ and $\operatorname{dim} \operatorname{Der}(\mathfrak{E})=8+3+3=$ 14. Let $\mathscr{H}$ be a Cartan subalgebra of $\mathscr{D}_{0} \cong \mathfrak{I}(3, C)$. Then $\mathscr{H}$ acts diagonally in $\operatorname{Der}\left(\mathfrak{S}^{5}\right)$ and has weights of the adjoint representation, the standard representation and the contragredient of this. It follows that $\mathscr{H}$ is a Cartan subalgebra of $\operatorname{Der}(\mathfrak{E})$ and the root system $\Delta$ of $\operatorname{Der}(\Subset)$ w.r.t. $\mathscr{H}$ is given by

$$
\Delta=\left\{ \pm \lambda_{1}, \pm \lambda_{2}, \pm\left(\lambda_{1}+\lambda_{2}\right), \pm\left(\lambda_{1}-\lambda_{2}\right), \pm\left(\lambda_{1}+2 \lambda_{2}\right), \pm\left(2 \lambda_{1}+\lambda_{2}\right)\right\}
$$

Squaring these and adding we obtain $\langle h, h\rangle_{\operatorname{Der}(\mathbb{E})}=16\left(\lambda_{1}^{2}+\lambda_{1} \lambda_{2}+\lambda_{2}^{2}\right)$ for $h=h\left(\lambda_{1}, \lambda_{2}\right) \in \mathscr{H}$ where $\langle,\rangle_{\operatorname{Der}(\mathbb{E})}$ is the Killing form of $\operatorname{Der}(\mathbb{S})$. Thus we

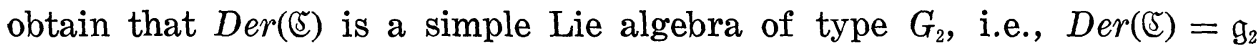
(see Example 30), and $\mathfrak{S}_{0}=\{a \in \mathfrak{S} \mid \operatorname{tr} \alpha=\alpha+\bar{a}=0\}$ is a 7-dimensional irreducible representation space of $\mathfrak{g}_{2}=\operatorname{Der}(\mathfrak{E})$. Let $\mathscr{J}$ be the exceptional simple Jordan algebra and $\mathscr{J}_{0}=\{X \in \mathscr{J} \mid \operatorname{Tr} X=0\}$. As we saw in Example $38, \mathscr{J}_{0}$ is a 26-dimensional irreducible representation space of $F_{4}=\operatorname{Der}(\mathscr{J})$. We are now ready to define Lie algebra $g=\operatorname{Der}(\mathfrak{E}) \oplus \mathfrak{夭}_{0} \otimes \mathscr{J}_{0} \oplus \operatorname{Der}(\mathscr{J})$ of dimension $14+7 \times 26+52=248$. We require $\operatorname{Der}(\mathfrak{E}) \oplus \operatorname{Der}(\mathscr{J})$ the Lie algebra direct sum of $\operatorname{Der}(\mathbb{E})$ and $\operatorname{Der}(\mathscr{J})$ to be a subalgebra of $\mathrm{g}$. We define that $[a \otimes x, D+E]=(D a) \otimes x+a \otimes(E x)$ for $D \in \operatorname{Der}(\mathfrak{E})$, $E \in \operatorname{Der}(\mathscr{J}), a \in \mathfrak{S}_{0}, x \in \mathscr{J}_{0}$. Finally we require $[a \otimes x, b \otimes y]=\frac{1}{12} T(x, y) D_{a, b}$ $+(a * b) \otimes(x * y)+\frac{1}{2} t(a, b)\left[R_{X}, R_{Y}\right], a, b \in \mathfrak{L}_{0}, x, y \in \mathscr{J}_{0}$ where $a * b=a b-$ $\frac{1}{2} t(a, b) \cdot 1, x * y=x \circ y-\frac{1}{3} T(x, y) \cdot 1, t(a, b)=\operatorname{tr} a b, T(x, y)=\operatorname{Tr} x \circ y$. Then one can check that this defines the Lie algebra structure in $\mathfrak{g}$ (see [5]). The Killing form $B$ of $\mathfrak{g}$ is given by $B\left(D+a \otimes x+E, D^{\prime}+b \otimes y+E^{\prime}\right)$ $=\frac{15}{2}\left\langle D, D^{\prime}\right\rangle_{D e r(\Subset)}+15 t(a, b) T(X, Y)+\frac{10}{3}\left\langle E, E^{\prime}\right\rangle_{\operatorname{Der}(\S)}, D, D^{\prime} \in \operatorname{Der}\left(\right.$ (ङ) $, E, E^{\prime}$ 
$\in \operatorname{Der}(\mathscr{J}), a, b \in \mathfrak{S}_{0}, x, y \in \mathscr{J}_{0}$ where $\langle,\rangle_{\operatorname{Der}(\mathbb{E})}$ and $\langle,\rangle_{\operatorname{Der}(\mathscr{g})}$ is the Killing form of $\mathrm{g}_{2}=\operatorname{Der}(\mathfrak{E})$ and $F_{4}=\operatorname{Der}(\mathscr{J})$ respectively. Let $\mathfrak{M}_{0}=\left(\sum_{i=1}^{3} C E_{i}\right)$ $\cap \mathscr{J}_{0}, \mathfrak{n}_{0}=\boldsymbol{C}\left(f_{1}-f_{2}\right)$ and put $\mathfrak{h}=\mathfrak{h}_{g_{2}} \oplus \mathfrak{n}_{0} \otimes \mathfrak{M}_{0} \oplus \mathfrak{h}_{F_{4}}$ where $\mathfrak{h}_{g_{2}}, \mathfrak{h}_{F_{4}}$ are Cartan subalgebras of $\mathfrak{g}_{2}=\operatorname{Der}(\mathfrak{E})$ and $F_{4}=\operatorname{Der}(\mathscr{J})$. Then $\mathfrak{h}$ is a Cartan subalgebra of g. Let $\left\{H_{1}, H_{2}, H_{3}, H_{4}\right\}$ be a basis for $\mathfrak{h}_{F_{4}}$ such that $H\left(\lambda_{1}, \lambda_{2}, \lambda_{3}, \lambda_{4}\right)=\sum_{i=1}^{4} \lambda_{i} H_{i}$ in Example 38, $\left\{H_{5}, H_{6}\right\}$ a basis for $\mathfrak{h}_{82}$ as in Example 30, and put $H_{7}=\left(f_{1}-f_{2}\right) \otimes\left(E_{1}-E_{3}\right), H_{8}=\left(f_{1}-f_{2}\right) \otimes\left(E_{2}-E_{3}\right)$. Then $\left\{H_{1}, \cdots, H_{8}\right\}$ is a basis of $\mathfrak{h}$, and if we denote an element $\sum \lambda_{i} H_{i}$ $\mapsto \sum a_{i} \lambda_{i}$ of $\mathfrak{h}^{*}$ by $\sum a_{i} \lambda_{i}$, the root system $\Delta$ of $\mathrm{g}$ w.r.t.h is given by $\Delta=\left\{ \pm \lambda_{i} \pm \lambda_{j} i<j=1,2,3,4 ; \pm\left(\lambda_{5}-\lambda_{6}\right), \pm\left(\lambda_{5}+2 \lambda_{6}\right), \pm\left(2 \lambda_{5}+\lambda_{6}\right) ; \pm \lambda_{i} \pm\right.$ $\frac{2 \lambda_{7}+\lambda_{8}}{2}, \pm \Lambda_{i}^{\prime} \pm \frac{\lambda_{7}-\lambda_{8}}{2}, \pm \Lambda_{i}^{*} \pm \frac{\lambda_{7}+2 \lambda_{8}}{2} i=1,2,3,4 ; \pm\left(\lambda_{5}-\lambda_{7}\right), \pm\left(\lambda_{5}\right.$ $\left.-\lambda_{8}\right), \pm\left(\lambda_{5}+\lambda_{7}+\lambda_{8}\right), \pm\left(\lambda_{6}-\lambda_{7}\right), \pm\left(\lambda_{6}-\lambda_{8}\right), \pm\left(\lambda_{6}+\lambda_{7}+\lambda_{8}\right), \pm\left(\lambda_{5}+\lambda_{6}+\lambda_{7}\right)$, $\pm\left(\lambda_{5}+\lambda_{6}+\lambda_{8}\right), \pm\left(\lambda_{5}+\lambda_{6}-\lambda_{7}-\lambda_{8}\right) ; \pm \lambda_{i} \pm\left(\lambda_{5}+\frac{\lambda_{8}}{2}\right), \pm \lambda_{i} \pm\left(\lambda_{6}+\frac{\lambda_{8}}{2}\right), \pm \lambda_{i}$ $\pm\left(\lambda_{5}+\lambda_{6}-\frac{\lambda_{8}}{2}\right), \pm \Lambda_{i}^{\prime} \pm\left(\lambda_{5}-\frac{\lambda_{7}+\lambda_{8}}{2}\right), \pm \Lambda_{i}^{\prime} \pm\left(\lambda_{6}-\frac{\lambda_{7}+\lambda_{8}}{2}\right), \pm \Lambda_{i}^{\prime} \pm\left(\lambda_{5}+\right.$ $\left.\lambda_{6}+\frac{\lambda_{7}+\lambda_{8}}{2}\right), \pm \Lambda_{i}^{*} \pm\left(\lambda_{5}+\frac{\lambda_{7}}{2}\right), \pm \Lambda_{i}^{*} \pm\left(\lambda_{6}+\frac{\lambda_{7}}{2}\right), \pm \Lambda_{i}^{*} \pm\left(\lambda_{5}+\lambda_{6}-\frac{\lambda_{7}}{2}\right)$ $i=1,2,3,4\}$ where $\Lambda_{1}^{\prime}, \Lambda_{i}^{*}$ are as in Example 28. (See [5], but there are mistakes about the roots of $E_{8}$ in p. $102 \sim 103$ of [5].)

Put $\alpha_{1}=\frac{1}{2}\left\{\lambda_{1}-\lambda_{2}-\lambda_{3}-\lambda_{4}-\lambda_{7}-\lambda_{8}\right\}-\lambda_{5}-\lambda_{6}, \alpha_{2}=\lambda_{6}+\lambda_{7}+\lambda_{8}, \alpha_{3}$ $\lambda_{5}-\lambda_{6}, \alpha_{4}=\lambda_{6}-\lambda_{8}, \alpha_{5}=\lambda_{4}-\lambda_{5}-\lambda_{6}+\frac{\lambda_{8}}{2}, \alpha_{6}=\lambda_{3}-\lambda_{4}, \alpha_{7}=\lambda_{2}-\lambda_{3}, \alpha_{8}=\lambda_{6}$ $-\lambda_{7}$. Then $\Pi=\left\{\alpha_{1}, \cdots, \alpha_{8}\right\}$ is a fundamental root system. For example, $\lambda_{1}+\lambda_{2}=2 \alpha_{1}+4 \alpha_{2}+6 \alpha_{3}+5 \alpha_{4}+4 \alpha_{5}+3 \alpha_{6}+2 \alpha_{7}+3 \alpha_{8}$. Since $B\left(\sum \lambda_{i} H_{i}\right.$, $\left.\sum \lambda_{i} H_{i}\right)=60\left(\lambda_{1}^{2}+\lambda_{2}^{2}+\lambda_{3}^{2}+\lambda_{4}^{2}\right)+120\left(\lambda_{5}^{2}+\lambda_{5} \lambda_{6}+\lambda_{6}^{2}\right)+60\left(\lambda_{7}^{2}+\lambda_{7} \lambda_{8}+\lambda_{8}^{2}\right)$, we obtain that $\left(\sum m_{i} \lambda_{i}, \sum m_{i} \lambda_{i}\right)=\frac{1}{60}\left(m_{1}^{2}+m_{2}^{2}+m_{3}^{2}+m_{4}^{2}\right)+\frac{1}{90}\left(m_{5}^{2}-m_{5} m_{6}+\right.$ $\left.m_{6}^{2}\right)+\frac{1}{45}\left(m_{7}^{2}-m_{7} m_{8}+m_{8}^{2}\right)$. In particular, $\left(\alpha_{i}, \alpha_{i}\right)=\frac{1}{30}$ for $1 \leq i \leq 8$, $\left(\alpha_{i}, \alpha_{i+1}\right)=-\frac{1}{60}$ for $1 \leq i \leq 6, \quad\left(\alpha_{i}, \alpha_{j}\right)=0$ for $|i-j| \geqq 2$ except for $\left(\alpha_{3}, \alpha_{8}\right)=-\frac{1}{6} \overline{0}$. This shows that the Dynkin diagram of $\mathfrak{g}$ is

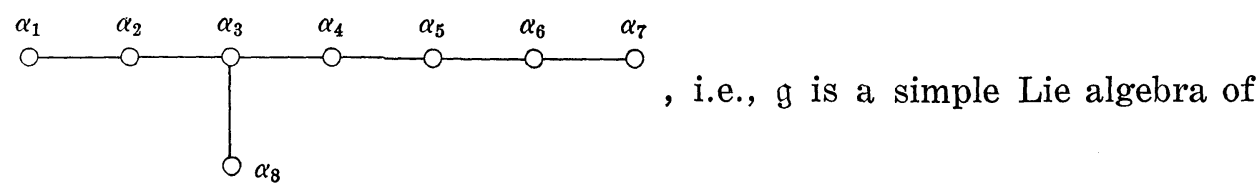

type $E_{8}$. Since we have $\Delta$ and $\Pi$, we have 120 positive roots $\Delta_{+}$and 
$\rho=\frac{1}{2} \sum_{\alpha \in \Delta_{+}} \alpha=46 \alpha_{1}+91 \alpha_{2}+135 \alpha_{3}+110 \alpha_{4}+84 \alpha_{5}+57 \alpha_{6}+29 \alpha_{7}+68 \alpha_{8}$.

Let $\Lambda_{1}, \cdots, \Lambda_{8}$ be the fundamental weights of $E_{8}$, i.e., $\frac{2\left(\Lambda_{i}, \alpha_{j}\right)}{\left(\alpha_{j}, \alpha_{j}\right)}=\delta_{i j}$, $i, j=1, \cdots, 8$. Let $\Lambda=\sum_{i=1}^{8} m_{i} \Lambda_{i}$ ( $m_{i} \geqq 0$, integer) be any dominant integral form. Then

$$
\left(\Lambda+\rho, \alpha_{i}\right)=\frac{m_{i}}{2}\left(\alpha_{i}, \alpha_{i}\right)+\left(\rho, \alpha_{i}\right)=\frac{1}{60}\left(m_{i}+1\right),
$$

$\left(\rho, \alpha_{i}\right)=\frac{1}{60}$ for $1 \leq i \leq 8$. The Weyl's dimension formula says that

$$
d(\Lambda)=\prod_{\alpha \in \Delta_{+}} \frac{(\Lambda+\rho, \alpha)}{(\rho, \alpha)}=\prod_{\sum_{i=1}^{8} n_{i} \alpha_{i} \in \Delta_{+}} \frac{\sum_{i=1}^{8} n_{i}\left(m_{i}+1\right)}{\sum_{i=1}^{8} n_{i}} .
$$

For example $d\left(\Lambda_{1}\right)=3875, d\left(\Lambda_{2}\right)=6696000, d\left(\Lambda_{3}\right)=6899079264, d\left(\Lambda_{4}\right)=$ $146325270, d\left(\Lambda_{5}\right)=2450240, d\left(\Lambda_{6}\right)=30380, d\left(\Lambda_{7}\right)=248, d\left(\Lambda_{8}\right)=147250$, $d\left(2 \Lambda_{7}\right)=27000, d\left(3 \Lambda_{7}\right)=1763125$, etc. This shows that the least dimensional irreducible representation of $E_{8}$ is the adjoint representation $\Lambda_{7}$.

Proposition 42. Let $g$ be a simple Lie algebra over $C$ and let $d \rho: \mathfrak{g} \rightarrow \mathfrak{g l}(V)$ be any representation of $\mathfrak{g}$ on $V$ with $g=\operatorname{dim} \mathfrak{g}$ and $d=$ $\operatorname{dim} V>1$. Then we have $g \leq \frac{1}{2} d(d+1)$ except when $g \cong \mathfrak{I l}(d, C)$.

Proof. Jf $\mathfrak{g}$ is of type $A_{n-1}(n \geqq 2)$, we have $d(\Lambda) \geqq d\left(\Lambda_{2}\right)=d\left(\Lambda_{n-2}\right)$ $\frac{1}{2} n(n-1) \geqq 2(n-1) \quad(n \geqq 4) \quad$ and $\quad d(\Lambda) \geqq d\left(2 \Lambda_{1}\right)=\frac{1}{2} n(n+1) \geqq 2(n-1)$ $(n=2,3)$ for any $\Lambda \neq \Lambda_{1}, \Lambda_{n-1}$, and hence, $\frac{1}{2} d(d+1) \geqq(n-1)(2 n-1) \geqq$ $n^{2}-1=g$ for $d=d(\Lambda), \Lambda \neq \Lambda_{1}, \Lambda_{n-1}$. If $\Lambda=\Lambda_{1}$ or $\Lambda=\Lambda_{n-1}$, we have $d=d\left(\Lambda_{1}\right)=d\left(\Lambda_{n-1}\right)=n$ and hence $\mathrm{g} \cong \mathfrak{g l}(d, C)$. If $\mathrm{g}$ is of type $C_{n}$, we have $d(\Lambda) \geqq d\left(\Lambda_{1}\right)=2 n$ for any $\Lambda$, and hence $\frac{1}{2} d(d+1) \geqq n(2 n+1)=g$. If $\mathrm{g}=\mathfrak{o}(n, C) \quad(n \geqq 5)$, we have $d(\Lambda) \geqq d\left(\Lambda_{1}\right)=n$ for any $\Lambda$ and hence $\frac{1}{2} d(d+1) \geqq \frac{1}{2} n(n+1) \geqq \frac{1}{2} n(n-1)=g$. If $g$ is of type $G_{2}$ (resp. $F_{4}, E_{6}$, $E_{7}, E_{8}$ ), we have seen that the least representation degree is 7 (resp. 26, 27, 56,248 ) and $g=\operatorname{dim} g$ is 14 (resp. 52,78,133,248), hence $\frac{1}{2} d(d+1) \geqq g$.

Q.E.D.

Remark 43. If $d=2$, then $\mathfrak{g}=\mathfrak{s l}(2, C), d \rho=\Lambda_{1}$. If $d=3$, then $\mathfrak{g}=\operatorname{sil}(2, C), d \rho=2 \Lambda_{1}$, or $\mathfrak{g}=\mathfrak{s} l(3, C), d \rho=\Lambda_{1}$ or its contragredient $\Lambda_{2}$.

DEFinition 44 . Let $V$ be a $n$-dimensional vector space over $C$. Then all non-singular endomorphisms of $V$ form a group $G L(V)$. By fixing a basis of $V$, we may identity $G L(V)$ with the group $G L(n, C)$ of all nonsingular $n \times n$ matrices, which is called the general linear group. 
subgroup $G$ of $G L(n, C)$ is called a linear algebraic group if there exists a finite number of polynomials $P_{1}, \cdots, P_{\mu}$ on $M(n, C)$ such that the group $G$ is the intersection of $G L(n, C)$ and the common zeros of these polynomials. In this case, the tangent space $\mathrm{g}$ of $G$ at the unit matrix $I_{n}$, i.e.,

$$
\mathfrak{g}=\left\{\xi \in M(n, C) \mid \sum_{i, j} \xi_{i j}\left(\frac{\partial P_{k}}{\partial x_{i j}}\left(I_{n}\right)\right)=0 \quad \text { for } 1 \leq k \leq \mu\right\}
$$

is a Lie subalgebra of $\operatorname{gr}(n, C)$, which is called the Lie algebra of $G$. For example, the special linear group $S L(n, C)=\{A \in G L(n, C) \mid \operatorname{det} A-$ $1=0\}$ is a linear algebraic group and its Lie algebra $\mathfrak{g}$ is, by definition,

$$
\mathfrak{g}=\left\{\xi \in M(n, C) \mid \sum_{i, j} \xi_{i j}\left(\frac{\operatorname{det} X-1}{2 x_{i j}}\left(I_{n}\right)\right)=\sum_{i=1}^{n} \xi_{i i}=0\right\},
$$

i.e., $\mathfrak{g}=\mathfrak{g l}(n, C)$ (see Example 21). The orthogonal group $O(n, C)=\{A \in$ $\left.M(n, C) \mid{ }^{t} A A=I_{n}\right\}$ and the special orthogonal group $S O(n, C)=\{A \in$ $O(n, C) \mid \operatorname{det} A=1\}$ are linear algebraic groups and their Lie algebras are the same as $\mathfrak{o}(n, C)$ (see Example 27). The Lie algebra of the symplectic group $S p(n, \boldsymbol{C})=\left\{\left.A \in G L(2 n, \boldsymbol{C})\right|^{t} A J A=J\right\}$ is a simple Lie algebra $\mathfrak{Z p}(n, \boldsymbol{C})$ of type $C_{n}$, where $J$ is defined as in Example 25. Here $S L(n, C)$ and $S p(n, C)$ are connected and simply connected, $S O(n, C)$ is connected but not simply connected, and $O(n, C)$ is not connected. We shall construct the spin group $\operatorname{Spin}(n, C)$ in $\S 5$, which is connected and simply connected and whose tie algebra is isomorphic to $\mathfrak{o}(n, C)$.

In general, the Lie algebras of two linear algebraic groups $G_{1}$ and $G_{2}$ are isomorphic if and only if $G_{1}$ and $G_{2}$ are locally isomorphic, and in this case we write $G_{1} \sim G_{2}$. We say that a connected linear algebraic group is almost simple when its Lie algebra $g$ is simple. Note that an almost simple algebraic group might have the center of finite numbers.

Let $\rho: G \rightarrow G L(V)$ be a representation of a linear algebraic group $G$ on $V$. Let $g$ be the Lie algebra of $G$. Then $\exp t X(t \in C, X \in \mathfrak{g})$ is in $G$ and there exists a representation $d \rho: g \rightarrow g r(V)$ of $g$ on $V$ defined by $d \rho(X)=\lim _{t \rightarrow 0} \frac{1}{t}(\rho(\exp t X)-1)$, i.e., $\rho(\exp t X)=\exp t d \rho(X)$ for $X \in \mathfrak{g}$. This representation $d \rho$ is called the infinitesimal (or differential) representation of $\rho$. Assume that $G$ is connected. Then $\rho$ is irreducible if and only if $d \rho$ is irreducible. Moreover, two representations of $G$ are equivalent if and only if their infinitesimal representations of $g$ are 
equivalent. Conversely, if $G$ is connected and simply connected, for any given representation $d \rho$ of $g$, there exists a representation $\rho$ of $G$ uniquely such that its infinitesimal representation is $d \rho$. For example, there is no representation of $S O(n, C)$ corresponding to the (half-) spin representation of $\mathfrak{D}(n, C)$ (see Example 27, 28), and we have to consider the spin group $\operatorname{Spin}(n, C)$.

ExAMPle 45 . Let $g$ be a Lie algebra over $\boldsymbol{C}$. Then there exists, uniquely up to isomorphism, a connected and simply connected linear algebraic group $G$ with the Lie algebra g. For example, since the Dynkin diagrams of type $A_{3}$ and $D_{3}$ are the same as $\bigcirc-\bigcirc-\circ$, the corresponding connected and simply connected algebraic groups $S L(4, C)$ and $\operatorname{Spin}(6, C)$ are isomorphic. Two inequivalent half-spin representations of $\operatorname{Spin}(6, C)$ correspond to the standard representation $\Lambda_{1}$ and its contragredient representation $\Lambda_{3}$ of $S L(4, C)$. The representation $\Lambda_{2}(=\square)$ of $S L(4, C)$ has a kernel $\{ \pm 1\}$ and its image is $S O(6, C)$ since its image leaves the Pfaffian of $4 \times 4$-skew symmetric matrices invariant (see Definition 22, (7)). This fact corresponds to the exact sequence $1 \rightarrow\{ \pm 1\}$ $\rightarrow \operatorname{Spin}(6, C) \rightarrow S O(6, C) \rightarrow 1$ (see (5.27)). Similarly the Dynkin diagrams of $B_{2}$ and $C_{2}$ are the same as $\bigcirc \Longrightarrow \circ$, the corresponding connected and simply connected algebraic group $\operatorname{Spin}(5, C)$ and $S p(2, C)$ are isomorphic. The restriction of $\Lambda_{1}$ (and also of $\Lambda_{3}$ ) of $S L(4, C)$ to $S p(2, C)$ is $\Lambda_{1}$ of $S p(2, C)$ which is corresponding to the spin representation of $\operatorname{Spin}(5, C)$.

The representation $\Lambda_{2}\left(=-, d\left(\Lambda_{2}\right)=5\right)$ of $S p(2, C)$ has a kernel $\{ \pm 1\}$ and its image is $S O(5, C)$. This fact is corresponding to the exact sequence $1 \rightarrow\{ \pm 1\} \rightarrow \operatorname{Spin}(5, C) \rightarrow S O(5, C) \rightarrow 1$. Since the Dynkin diagram of $\mathfrak{D}(4, C)$ and $\mathfrak{g}(2, C) \oplus \mathfrak{g l}(2, C)$ are the same as $\bigcirc \Longrightarrow 0$, we have the isomorphism $\operatorname{Spin}(4, C) \cong S L(2, C) \times S L(2, C)$. The two representations $\Lambda_{1} \otimes 1,1 \otimes \Lambda_{1}$ (i.e., $\left.\square \otimes 1,1 \otimes \square\right)$ of $S L(2, C) \times S L(2, C)$ are corresponding to the two inequivalent half-spin representations of $\operatorname{Spin}(4, C)$. Let $V$ be all $2 \times 2$ matrices $M(2, C)$. Define $\rho: S L(2) \times S L(2) \rightarrow G L(V)$ by $X \mapsto A X^{t} B$ for $X \in V,(A, B) \in S L(2) \times S L(2)$. Then $\rho=\Lambda_{1} \otimes \Lambda_{1}(=\square \otimes \square)$ and its kernel is $\{ \pm 1\}$. The image is $S O(4, C)$ since it leaves $\operatorname{det} X(X \in V)$ invariant. This fact is corresponding to the exact sequence $1 \rightarrow\{ \pm 1\} \rightarrow$ $\operatorname{Spin}(4, C) \rightarrow S O(4, C) \rightarrow 1$. Since the Dynkin diagrams of $A_{1}, B_{1}, C_{1}$ are the same as 0 , we have $S L(2, C) \cong \operatorname{Spin}(3, C) \cong S p(1, C)$. Under the 
isomorphism $\operatorname{Spin}(4, C) \cong S L(2) \times S L(2)$, the subgroup $\{(A, A) \in S L(2) \times$ $S L(2) \mid A \in S L(2)\} \cong S L(2)$ corresponds to $\operatorname{Spin}(3, C)$. Thus the restriction of $\Lambda_{1} \otimes 1$ (and $1 \otimes \Lambda_{1}$ ) to that subgroup $\cong S L(2, C)$ is $\Lambda_{1}$ which corresponds to the spin representation of $\operatorname{Spin}(3, C)$. The representation $2 \Lambda_{1}$ of $S L(2, C)$ has a kernel $\{ \pm 1\}$ and the image $S O(3, C)$. This fact is corresponding to the exact sequence $1 \rightarrow\{ \pm 1\} \rightarrow \operatorname{Spin}(3, C) \rightarrow \operatorname{SO}(3, C) \rightarrow 1$.

DEFINITION 46. Let $\rho: S L(n, C) \rightarrow G L(V)$ be an irreducible representation of $G$ on $V$ with the highest weight $\Lambda$. Then there exists canonically a representation $\rho^{\prime}: G L(n, C) \rightarrow G L(V)$ of $G L(n, C)$ such that the restriction of $\rho^{\prime}$ to $S L(n, C)$ is $\rho$. In this case we say that the highest weight of $\rho^{\prime}$ is $\Lambda$. We also apply the Young diagram to $G L(n, C)$.

\section{§2. Castling transforms}

DEFINITION 1. Let $G$ be a connected linear algebraic group, $V$ a finite dimensional vector space $(\operatorname{dim} V \geqq 1$ ), and $\rho$ a rational representation of $G$ on $V$, all defined over the complex number field $C$. We call a triplet $(G, \rho, V)$ a prehomogeneous vector space (abbrev. P. V.) when there exists a proper algebraic subset $S$ of $V$ such that $V-S$ consists of a single $G$-orbit. In this case, points of $S$ (resp. $V-S$ ) are called singular (resp. generic) points. Let $g$ be the Lie algebra of $G$ and let $d \rho: \mathfrak{g} \rightarrow \mathfrak{g l}(V)$ be the infinitesimal representation of $\rho$. For an element $x$ of $V$, the Lie algebra of the isotropy subgroup $G_{x}=\{g \in G \mid \rho(g) x=x\}$ of $G$ at $x$ is the isotropy subalgebra $\mathfrak{g}_{x}=\{A \in \mathfrak{g} \mid d \rho(A) x=0\}$ of $g$ at $x$.

Proposition 2. The following conditions are equivalent.

(1) A triplet $(G, \rho, V)$ is a P.V.

(2) There exists an element $x$ of $V$ satisfying $\operatorname{dim} G_{x}=\operatorname{dim} G-$ $\operatorname{dim} V$, i.e., $\operatorname{dim} \mathfrak{g}_{x}=\operatorname{dim} \mathfrak{g}-\operatorname{dim} V$.

Proof. (1) $\Rightarrow(2)$ : Let $x$ be a generic point. Then we have $V-S$ $=\rho(G) \cdot x \approx G / G_{x}$ and hence $\operatorname{dim} G-\operatorname{dim} G_{x}=\operatorname{dim}(V-S)=\operatorname{dim} V$.

$(2) \Rightarrow(1): \quad$ In general, we have $\rho(G) \cdot x=\overline{\rho(G) \cdot x}-\overline{(\rho(G) \cdot x}-\rho(G) \cdot x)$ where denotes the Zariski closure. The second condition implies that $\operatorname{dim} \overline{\rho(G) \cdot x}=\operatorname{dim} \rho(G) \cdot x=\operatorname{dim} V$. Since $V$ is irreducible, we have $V=$ $\overline{\rho(G) \cdot x}$ and $\rho(G) \cdot x=V-S$ where $S=\overline{(\rho(G) \cdot x}-\rho(G) \cdot x)$.

Q.E.D.

Proposition 2 implies that the prehomogeneity of a triplet is an infinitesimal condition. 
Proposition 3. Let $(G, \rho, V)$ be a triplet. Assume that there exists a non-constant rational function $f(x)$ satisfying $f(\rho(g) x)=f(x)$ for all $g \in G, x \in V$. Then this triplet $(G, \rho, V)$ is not a $P . V$.

Proof. Assume that a triplet $(G, \rho, V)$ is a P. V. and let $x_{0}$ be a generic point. Then $f(x)$ is constant on the open orbit $\rho(G) \cdot x_{0}$. Since $f(x)$ is a rational function, it is constant on the Zariski closure of $\rho(G) \cdot x_{0}$, i.e., on $V$.

Q.E.D.

These propositions will be used in $\S 5$ to investigate the prehomogeneity of a given triplet.

Definition 4. Two triplets $(G, \rho, V)$ and $\left(G^{\prime}, \rho^{\prime}, V^{\prime}\right)$ are called equivalent (or strongly equivalent) if there exist a rational isomorphism $\sigma: \rho(G)$ $\rightarrow \rho^{\prime}\left(G^{\prime}\right)$ and an isomorphism $\tau: V \rightarrow V^{\prime}$, both defined over $C$ such that the following diagram is commutative for all $g \in G$. This equivalence

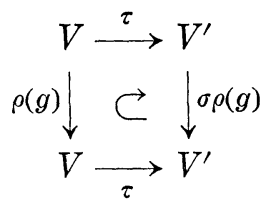

relation will be denoted by $(G, \rho, V) \cong\left(G^{\prime}, \rho^{\prime}, V^{\prime}\right)$. Note that we consider only the image $\rho(G)$, not $G$ itself. For example, we have $\left(S L(4, C), \Lambda_{2}, V(6)\right)$ $\cong\left(S O(6, C), \Lambda_{1}, V(6)\right)$ although $S L(4, C)$ and $S O(6, C)$ are not isomorphic (see Example $45, \S 1)$. A triplet $(G, \rho, V)$ is called irreducible when $\rho$ is irreducible. In this case, by Theorem 1 in $\S 1$, the Lie algebra $g$ of the image $\rho(G)$ is reductive, with center at most one-dimensional. In particular, a triplet $(G, \rho, V)$ is equivalent to its dual $\left(G, \rho^{*}, V^{*}\right)$ where $\rho^{*}$ is the contragredient representation of $\rho$ on the dual vector space $V^{*}$ of $V$. In the following, except $\S 4$, we shall assume that a triplet is irreducible.

LEMMA 5. Let $\tilde{G}$ be a connected algebraic group and let $W, W^{\prime}$ be irreducible algebraic varieties on which $\tilde{G}$ acts. Let $f: W \rightarrow W^{\prime}$ be a generically surjective (i.e., $\overline{f(W)}=W^{\prime}$ ), $\tilde{G}$-equivariant morphism (i.e., compatible with the action of $\tilde{G})$. Then the following conditions are equivalent:

(i) $W$ is $\tilde{G}$-prehomogeneous, i.e., it has a Zariski-dense $\tilde{G}$-orbit.

(ii) $W^{\prime}$ is $\tilde{G}$-prehomogeneous, and for a point $x^{\prime}$ of a Zariski-dense 
orbit, $f^{-1}\left(x^{\prime}\right)$ is $\tilde{G}_{x^{\prime}}$-prehomogeneous, where $\tilde{G}_{x^{\prime}}$ is the isotropy subgroup of $\tilde{G}$ at $x^{\prime}$.

Definition 6. Let $(G, \rho, V)$ be a P. V. The isotropy subgroup $G_{x}$ of $G$ at a generic point $x \in V-S$ is called a generic isotropy subgroup. Note that all generic isotropy subgroups of $(G, \rho, V)$ are isomorphic to each other. Similarly, the isotropy subalgebra of a generic point is called a generic isotropy subalgebra. Since we consider everything over $C$, we shall denote $G L(n, C)$ (resp. $S L(n, C), O(n, C), S O(n, C), \operatorname{Spin}(n, C)$, $S p(n, C)$ ) by $G L(n)$ (resp. $S L(n), O(n), S O(n), S p i n(n), S p(n))$.

Let $G$ be a linear algebraic group, and let $\rho: G \rightarrow G L(V(m))$ be a faithful irreducible representation of $G$ on the $m$-dimensional vector space $V(m)$. Let $\rho^{*}: G \rightarrow G L\left(V(m)^{*}\right)$ be the contragredient representation of $\rho$ on the dual vector space $V(m)^{*}$ of $V(m)$, and let $n$ be a positive integer with $m>n \geqq 1$.

Proposition 7. A triplet $\left(G \times G L(n), \rho \otimes \Lambda_{1}, V(m) \otimes V(n)\right)$ is a P.V. if and only if a triplet $\left(G \times G L(m-n), \rho^{*} \otimes \Lambda_{1}, V(m)^{*} \otimes V(m-n)\right)$ is a $P . V$., and in this case, their generic isotropy subgroups are isomorphic to each other.

Proof. Identify $V=V(m) \otimes V(n)$ with $\overbrace{V(m) \oplus \cdots \oplus V(m)}^{n}$, and let $W$ be an algebraic variety whose points are vectors $v=\left(v_{1}, \cdots, v_{n}\right) \in V\left(v_{i} \in\right.$ $V(m)$ ) such that $v_{1}, \cdots, v_{n}$ are linearly independent in $V(m)$. Then the triplet $\left(G \times G L(n), \rho \times \Lambda_{1}, V(m) \otimes V(n)\right)$ is a P. V. if and only if $W$ is $\tilde{G}$ prehomogeneous for $\tilde{G}=G \times G L(n)$. Let $W^{\prime}$ be the Grassmann variety $\operatorname{Grass}_{n}(V(m))$ whose points are $n$-dimensional subspaces of $V(m)$. For an element $v=\left(v_{1}, \cdots, v_{n}\right)$ in $W$, let $f(v)$ be the $n$-dimensional subspace of $V(m)$ spanned by $v_{1}, \cdots, v_{n}$. Then $f: W \rightarrow W^{\prime}$ is a surjective, $\tilde{G}$-equivariant morphism. By Lemma $5, W$ is $\tilde{G}$-prehomogeneous if and only if $W^{\prime}=\operatorname{Grass}_{n}(V(m))$ is $\tilde{G}$-prehomogeneous, i.e., G-prehomogeneous since $G L(n)$ acts on $\operatorname{Grass}_{n}(V(m))$ trivially, and it acts on each fibre homogeneously. As $\operatorname{Grass}_{n}(V(m))$ is $G$-prehomogeneous if and only if $\operatorname{Grass}_{m-n}\left(V(m)^{*}\right)$ is $G$-prehomogeneous, again by Lemma 5 , that is so if and only if $\left(G \times G L(m-n), \rho^{*} \otimes \Lambda_{1}, V(m)^{*} \otimes V(m-n)\right)$ is a P. V., and thus we obtain our first assertion. Since each fibre is a principal homogeneous space of $G L(n)$, the generic isotropy subgroup of $(G \times G L(n)$, $\left.\rho \otimes \Lambda_{1}, V(m) \otimes V(n)\right)$ is isomorphic to that of $\left(G, \operatorname{Grass}_{n}(V(m))\right)$. As the 
generic isotropy subgroups of $\left(G, \operatorname{Grass}_{n}(V(m))\right)$ and $\left(G, \operatorname{Grass}_{m-n}(V(m) *)\right)$ are isomorphic, we get our second assertion.

Q.E.D.

LEMMA 8. Let $G$ be a connected semi-simple algebraic group and let $\rho: G \rightarrow G L(V)$ be an irreducible representation. Assume that the triplet $(G \times G L(1), \rho \otimes \square, V \otimes V(1))$ is a P.V. with the generic isotropy subgroup $H$. Then the triplet $(G, \rho, V)$ is a P.V., if and only if the connected component of $H$ is not contained in $G$.

Proof. The triplet $(G, \rho, V)$ is a P.V. if and only if $\operatorname{dim} G-\operatorname{dim} G \cap H$ $=\operatorname{dim} V(=\operatorname{dim} G \times G L(1)-\operatorname{dim} H)$, i.e., $\operatorname{dim} G \cap H=\operatorname{dim} H-1$. Since $\operatorname{dim} G \cap H=\operatorname{dim} H-1$ if and only if the connected component of $H$ is not contained in $G$, we obtain our assertion.

Q.E.D.

Proposition 9. Let $G$ be a linear algebraic group and let $\rho: G \rightarrow$ $G L(V(m))$ be a faithful irreducible representation of $G$ on the m-dimensional vector space $V(m)$. Let $n$ be a positive number with $m>n \geqq 1$. Then a triplet $\left(G \times S L(n), \rho \otimes \Lambda_{1}, V(m) \otimes V(n)\right)$ is a P.V. if and only if $\left(G \times S L(m-n), \rho^{*} \otimes \Lambda_{1}, V^{*}(m) \otimes V(m-n)\right)$ is a P.V., and in this case, their generic isotropy subgroups are isomorphic to each other.

Proof. Note that $G$ is reductive with at most one-dimensional center by Theorem 1 in $\S 1$. When $G$ has the one-dimensional center, our assertion is the same as Proposition 7 , and hence we may assume that $G$ is semi-simple. Assume that $\left(G \times S L(n), \rho \otimes \Lambda_{1}, V(m) \otimes V(n)\right)$ is a P.V. with the generic isotropy subgroup $H$. Then $\left(G \times G L(n), \rho \otimes \Lambda_{1}, V(m)\right.$ $\otimes V(n))$ is a P.V. and its generic isotropy subgroup is isomorphic to $H$ $\times G L(1)$ by Lemma 8 . Then Proposition 8 says that $(G \times G L(m-n)$, $\rho^{*} \otimes \Lambda_{1}, V(m)^{*} \otimes V(m-n)$ is a P.V. with the generic isotropy subgroup $H^{\prime} \cong H \times G L(1)$. Since $H^{\prime} \cap(G \times S L(m-n)) \cong H,\left(G \times S L(m-n), \rho^{*}\right.$ $\left.\otimes \Lambda_{1}, V(m)^{*} \otimes V(m-n)\right)$ is a P.V. by Lemma 8 .

Q.E.D.

This Proposition 9 is very important because it gives us a general method to obtain infinitely many new prehomogeneous vector spaces from a given prehomogeneous vector space. Let $(G, \rho, V)$ be any P.V. with $\operatorname{dim} V=m \geqq 2$. Then since $S L(1)=\{1\}$ and $V \otimes V(1) \cong V$, we have $(G$, $\rho, V) \cong\left(G \times S L(1), \rho \otimes \Lambda_{1}, V \otimes V(1)\right)$ and by Proposition 9 , we obtain a new P.V. $\left.(G \times S L(m-1)), \rho^{*} \otimes \Lambda_{1}, V^{*} \otimes V(m-1)\right)$. Since we may assume that $G$ is reductive, this P.V. is equivalent to $(G \times S L(m-1)$, $\rho$ $\left.\otimes \Lambda_{1}, V \otimes V(m-1)\right)$. Applying this procedure again to this new P.V., 
we obtain the second new P.V. $\left(G \times S L(m-1) \times S L\left(m^{2}-m-1\right), \rho \otimes \Lambda_{1}\right.$ $\left.\otimes \Lambda_{1}, V \otimes V(m-1) \otimes V\left(m^{2}-m-1\right)\right)$. Now there are two ways to apply Proposition 9 to this second new P.V., namely we have two new P.V. $\left(G \times S L\left(m^{2}-m-1\right) \times S L\left(m^{3}-m^{2}-2 m+1\right), \quad \rho \otimes \Lambda_{1} \otimes \Lambda_{1}, \quad V \otimes V\left(m^{2}-\right.\right.$ $\left.m-1) \otimes V\left(m^{3}-m^{2}-2 m+1\right)\right)$ and $\left(G \times S L(m-1) \times S L\left(m^{2}-m-1\right)\right.$ $\times S L\left(m^{4}-2 m^{3}+m-1\right), \rho \otimes \Lambda_{1} \otimes \Lambda_{1} \otimes \Lambda_{1}, V \otimes V(m-1) \otimes V\left(m^{2}-m-1\right)$ $\left.\otimes V\left(m^{4}-2 m^{3}+m-1\right)\right)$ where $m^{4}-2 m^{3}+m-1=m(m-1)\left(m^{2}-m-\right.$ 1) -1 . If $m \geqq 3$, these new P.V. are not equivalent to the original P.V. For example, a triplet ( $\left.S L(3), \Lambda_{1}, V(3)\right)$ is obviously a P.V. and hence $(S L(3)$ $\left.\times S L(2) \times S L(5) \times S L(29), \Lambda_{1} \otimes \Lambda_{1} \otimes \Lambda_{1} \otimes \Lambda_{1}, V(3) \otimes V(2) \otimes V(5) \otimes V(29)\right)$ is a P.V., etc. Repeating this procedure, we can obtain infinitely many new P.V.'s. Although these prehomogeneous vector spaces obtained from a given P.V. are in general not equivalent, they have many common properties. For example, their generic isotropy subgroups are isomorphic to the original one. Thus we attain the concept of castling transforms or castling classes of prehomogeneous vector spaces. It is convenient to define these concepts among irreducible triplets.

DeFinition 10. We say that two triplets $(G, \rho, V)$ and $\left(G^{\prime}, \rho^{\prime}, V^{\prime}\right)$ are castling transforms of each other when there exist a triplet $(\tilde{G}, \tilde{\rho}, V(m))$ and a positive number $n$ with $m>n \geqq 1$ such that

$$
(G, \rho, V) \cong\left(\tilde{G} \times S L(n), \tilde{\rho} \otimes \Lambda_{1}, V(m) \otimes V(n)\right)
$$

and

$$
\left(G^{\prime}, \rho^{\prime}, V^{\prime}\right) \cong\left(\tilde{G} \times S L(m-n), \tilde{\rho}^{*} \otimes \Lambda_{1}, V(m)^{*} \otimes V(m-n)\right)
$$

where $\tilde{\rho}^{*}$ is the contragredient representation of $\tilde{\rho}$ on the dual vector space $V(m) *$ of $V(m)$. A triplet $(G, \rho, V)$ is called reduced if there is no castling transform $\left(G^{\prime}, \rho^{\prime}, V^{\prime}\right)$ of $(G, \rho, V)$ with $\operatorname{dim} V^{\prime}<\operatorname{dim} V$.

DEFINITION 11. We say that two triplets $(G, \rho, V)$ and $\left(G^{\prime}, \rho^{\prime}, V^{\prime}\right)$ belong to the same castling class when one is obtained from the other by a finite number of castling transforms, and in this case we write $(G, \rho, V)$ $\sim\left(G^{\prime}, \rho^{\prime}, V^{\prime}\right)$.

We can obtain the reduced triplet from any given one by a finite number of successive castling transforms for the dimension reasons. For any given triplet, such a reduced one is unique; namely,

Proposition 12. Each castling class contains one and, up to strong equivalence relation, only one reduced triplet. 
Proof. Suppose that a triplet $(G, \rho, V)$ has at least two castling transforms $\left(G^{\prime}, \rho^{\prime}, V^{\prime}\right)$ and $\left(G^{\prime \prime}, \rho^{\prime \prime}, V^{\prime \prime}\right)$, then we may assume without loss of generality that there exists a triplet $(\tilde{G}, \tilde{\rho}, V(m))$ such that

$$
(G, \rho, V) \cong\left(\tilde{G} \times S L\left(n_{1}\right) \times S L\left(n_{2}\right), \tilde{\rho} \otimes \Lambda_{1} \otimes \Lambda_{1}, V(m) \otimes V\left(n_{1}\right) \otimes V\left(n_{2}\right)\right)
$$

with $m \geqq 2$, and

$$
\begin{aligned}
& \left(G^{\prime}, \rho^{\prime}, V^{\prime}\right) \cong\left(\tilde{G} \times S L\left(n_{1}\right) \times S L\left(m n_{1}-n_{2}\right),\right. \\
& \left.\tilde{\rho}^{*} \otimes \Lambda_{1}^{*} \otimes \Lambda_{1}, V(m)^{*} \otimes V\left(n_{1}\right) * \otimes V\left(m n_{1}-n_{2}\right)\right) \\
& \left(G^{\prime \prime}, \rho^{\prime \prime}, V^{\prime \prime}\right) \cong\left(\tilde{G} \times S L\left(n_{2}\right) \times S L\left(m n_{2}-n_{1}\right),\right. \\
& \left.\tilde{\rho}^{*} \otimes \Lambda_{1}^{*} \otimes \Lambda_{1}, \quad V(m)^{*} \otimes V\left(n_{2}\right) * \otimes V\left(m n_{2}-n_{1}\right)\right) .
\end{aligned}
$$

In this case $\operatorname{dim} V=m n_{1} n_{2}, \quad \operatorname{dim} V^{\prime}=m n_{1}\left(m n_{1}-n_{2}\right)$ and $\operatorname{dim} V^{\prime \prime}=$ $m n_{2}\left(m n_{2}-n_{1}\right)$. Assume that $\operatorname{dim} V^{\prime}<\operatorname{dim} V$ and $\operatorname{dim} V^{\prime \prime}<\operatorname{dim} V$. This implies that $m n_{1}<2 n_{2}$ and $m n_{2}<2 n_{1}$, and hence $m^{2}<2^{2}$, i.e., $m<2$, a contradiction. This shows that if there exists a castling transform $\left(G^{\prime}, \rho^{\prime}, V^{\prime}\right)$ of $(G, \rho, V)$ satisfying $\operatorname{dim} V^{\prime}<\operatorname{dim} V$, then it is unique. Since $\operatorname{dim} V<+\infty$, we obtain our assertion.

Q.E.D.

Proposition 12 implies that a reduced triplet $(G, \rho, V)$ satisfies the condition that $\operatorname{dim} V \leq \operatorname{dim} V^{\prime}$ whenever $(G, \rho, V) \sim\left(G^{\prime}, \rho^{\prime}, V^{\prime}\right)$. By a classification of irreducible prehomogeneous vector spaces, we mean the determination of all reduced irreducible prehomogeneous vector spaces up to strong equivalence relation. Note that in this paper we shall use essentially Lie algebras even if we use the terminology of groups for the convenience. Finally we shall show two propositions which are obtained from Lemma 5 as Proposition 7.

Let $G$ be a linear algebraic group and let $\rho: G \rightarrow G L(V(d))$ be a representation of $G$ on the $d$-dimensional vector space $V(d)$. By choosing a basis of $V(d)$, we may identify $V(d)$ with $C^{d}$. Thus we may consider $\rho(g)(g \in G)$ as a $d \times d$ matrix. Define the vector space $\Lambda^{2}(V(d)$ ) (resp. $S^{2}(V(d))$ ) as the all $d \times d$ skew-symmetric (resp. symmetric) matrices. Define the representation $\rho_{-}$(resp. $\rho_{\square}$ ) of $G$ on $\Lambda^{2}\left(V(d)\right.$ ) (resp. $S^{2}(V(d))$ ) by $X \mapsto \rho(g) X^{t} \rho(g)$ for $X \in \Lambda^{2}(V(d))$ (resp. $X \in S^{2}(V(d))$ ), $g \in G$. Then $\rho_{-j}, \rho_{I I}$ are up to equivalence uniquely determined by $\rho$.

Proposition 13. Assume that $2 n \geqq d$. Then a triplet $(\operatorname{Sp}(n) \times G$, 
$\left.\Lambda_{1} \otimes \rho, V(2 n) \otimes V(d)\right)$ is a P.V. if and only if the triplet $\left(G, \rho_{--}, \Lambda_{2}(V(d))\right.$ is a P.V.

Proof. Let $\langle$,$\rangle be a skew-symmetric bilinear form on V(2 n) \times V(2 n)$ which is left invariant under the action of $S p(n)$. Identify $V(2 n) \otimes V(d)$ with $W=\overbrace{V(2 n) \oplus \cdots \oplus V(2 n)}^{d}$ and for an element $v=\left(v_{1}, \cdots, v_{d} \in W\left(v_{i}\right.\right.$ $\in V(2 n)$ ), let $f(v)$ be a $d \times d$ skew-symmetric matrix with $(i, j)$-element $\left\langle v_{i}, v_{j}\right\rangle(i, j=1, \cdots, 2 n)$. Then $f(v)$ is an element of $W^{\prime}=\Lambda^{2}(V(d))$ and $f: W \rightarrow W^{\prime}$ is a generically surjective, $\tilde{G}$-equivariant morphism for $\tilde{G}=$ $S p(n) \times G$. By Lemma 5, $\left(S p(n) \times G, \Lambda_{1} \otimes \rho, V(2 n) \otimes V(d)\right)$ is a P.V. if and only if $W^{\prime}$ is $\tilde{G}$-prehomogeneous, i.e., $G$-prehomogeneous since each generic fibre is $S p(n)$-prehomogeneous and $S p(n)$ acts trivially on $W^{\prime}=\Lambda^{2}(V(d))$.

Q.E.D.

Proposition 14. Assume that $n \geqq d$. Then a triplet $(S O(n) \times G$, $\left.\Lambda_{1} \otimes \rho, V(n) \otimes V(d)\right)$ is a P.V. if and only if the triplet $\left(G, \rho_{\bar{\square},}, S^{2}(V(d))\right)$ is a P.V.

Proof. Let $\langle$,$\rangle be a symmetric bilinear form on V(n) \times V(n)$ which is left invariant under the action of $S O(n)$. Then the rest of a proof is the same as Proposition 13.

Q.E.D.

Proposition 13 and Proposition 14 will be used in $\S 3$.

§3. Classification of reduced triplets $(G, \rho, V)$ satisfying $\operatorname{dim} G \geq \operatorname{dim} V$

Proposition 1. If a triplet $(G, \rho, V)$ is a P.V., then we have

$$
\operatorname{dim} G \geq \operatorname{dim} V \text {. }
$$

Proof. By Proposition 2 in $\S 2$ we have $\operatorname{dim} G-\operatorname{dim} V=\operatorname{dim} G_{x} \geq 0$ for some $x$ in $V$.

Q.E.D.

According to this proposition we shall determine in this section all the irreducible reduced triplets $(G, \rho, V)$ satisfying $\operatorname{dim} G \geq \operatorname{dim} V$. By Theorem 1 in $\S 1$, the Lie algebra $g$ of $\rho(G)$ is reductive with at most one-dimensional center. We shall consider in $\S 6$ the case when $g$ is semi-simple. In this section we shall consider the case that the center of $\mathrm{g}$ is of one dimension. Then by Theorem 2 in $\S 1$, we may assume that a triplet $(G, \rho, V)$ is of the form: $G=G L(1) \times G_{1} \times \cdots \times G_{k}, \rho=\square \otimes$ $\rho_{1} \otimes \cdots \otimes \rho_{k}, V=V(1) \otimes V\left(d_{1}\right) \otimes \cdots \otimes V\left(d_{k}\right)$ with $d_{1} \geq d_{2} \geq \cdots \geq d_{k} \geq 2$, 
where each $G_{i}$ is a connected almost simple algebraic group, $\rho_{i}$ is an irreducible representation of $G_{i}$ on the $d_{i}$-dimensional vector space $V\left(d_{i}\right)$ $(1 \leq i \leq k)$, and $\square$ is the standard representation of $G L(1)$ on the onedimensional vector space $V(1)$.

Put $g_{i}=\operatorname{dim} G_{i}(1 \leq i \leq k)$. These notations such as $G_{i}, \rho_{i}, d_{i}, g_{i}, k$ will be used throughout this section. We shall denote by $\left(G_{2}\right)$ the exceptional simple algebraic group of type $G_{2}$ of dimension 14 to distinguish it from the second group $G_{2}$.

Proposition 1 implies:

$$
1+g_{1}+\cdots+g_{k} \geq d_{1} d_{2} \cdots d_{k} .
$$

We shall induce some inequalities from (3.1).

LEMMA 2. Let $n$ be a natural number, and let $a, c$ be any real numbers satisfying $a \leq c a^{n-1}-a$. Then

$$
\sum_{i=1}^{n} x_{i}^{2}-c \prod_{i=1}^{n} x_{i} \leq n a^{2}-c a^{n}
$$

holds for any real numbers $x_{\nu}$ with $a \leq x_{\nu} \leq c a^{n-1}-a(\nu=1, \cdots, n)$.

Proof. Let $M$ be the maximum value of

$$
f\left(x_{1}, \cdots, x_{n}\right)=\sum_{i=1}^{n} x_{i}^{2}-c \prod_{i=1}^{n} x_{i}
$$

on the closed interval $[a, b]^{n}$, where $b=c a^{n-1}-a$. Since $f$ is quadratic in each variable with a positive leading coefficient, the maximum is attained at the boundary points. Hence if $M_{\mu}(1 \leq \mu \leq n)$ denotes the value of $f$ at those points where $x_{i}=a$ for $\mu$ distinct indices $i$ and $x_{j}=b$ for $n-\mu$ distinct indices $j$, then $M=\max _{1 \leq \mu \leq n} M_{\mu}$. We have $M_{n}=n a^{2}-c a^{n}$, and $M_{n}-M_{\mu}=-(n-\mu)\left(b^{2}-a^{2}\right)+c a^{\mu}\left(b^{n-\mu}-a^{n-\mu}\right)$. If $a=b$, then $M_{\mu}$ $=M_{n}$ for all $\mu$. On the other hand, if $a<b$, then we have $\left(M_{n}-M_{\mu}\right) /(b$ $-a) \geq-(n-\mu)(b+a)+(n-\mu) c a^{n-1}=(n-\mu)\left(c a^{n-1}-a-b\right)=0$, and hence $M_{\mu} \leq M_{n}$ for all $\mu$. This implies that $M=M_{n}=n a^{2}-c a^{n}$.

Q.E.D.

Proposition 3. Assume that a triplet $(G, \rho, V)$ is a P.V. with $2^{k-2} d_{1}$ $-2 \geq d_{2}$. Then we have

$$
1+g_{1} \geq 2^{k-1} d_{1}-3(k-1)
$$


Proof. Since the image $\rho_{i}\left(G_{i}\right)$ of the simple algebraic group $G_{i}$ is contained in $S L\left(d_{i}\right)$, we have $d_{i}^{2}-1 \geq g_{i}$ for $2 \leq i \leq k$, and hence from (3.1), we get

$$
1+g_{1} \geq(k-1)-\left(d_{2}^{2}+\cdots+d_{k}^{2}-d_{1} d_{2} \cdots d_{k}\right) .
$$

Putting $x_{1}=d_{2}, \cdots, x_{n}=d_{k}, n=k-1, c=d_{1}, a=2$ in Lemma 2, we have the inequality

$$
\begin{aligned}
d_{2}^{2}+ & \cdots+d_{k}^{2}-d_{1} d_{2} \cdots d_{k} \\
& \leq(k-1) 2^{2}-d_{1} \cdot 2^{k-1} \quad\left(2 \leq d_{i} \leq 2^{k-2} d_{1}-2\right) .
\end{aligned}
$$

From (3.2) and (3.3), we obtain the desired inequality.

Q.E.D.

Proposition 4. If a triplet $(G, \rho, V)$ is a P.V. with $k \geq 3$, then the following inequality holds.

$$
1+g_{1} \geq 2^{k_{0}-1} d_{1}-3\left(k_{0}-1\right) \quad \text { for } k \geq k_{0} \geq 3 .
$$

In particular, we have

$$
1+g_{1} \geq 4 d_{1}-6 .
$$

Proof. Since $k \geq 3$, we have $2^{k-2} d_{1}-2 \geq d_{2}$, i.e., the assumption of Proposition 3 is satisfied. Put $f(k)=2^{k-1} d_{1}-3(k-1)$. Then we have $f(k)-f\left(k_{0}\right)=\left(2^{k-1}-2^{k_{0}-1}\right) d_{1}-3\left(k-k_{0}\right) \geq 8\left(2^{k-k_{0}}-1\right)-3\left(k-k_{0}\right) \geq 0$ for $k \geq k_{0} \geq 3$, and hence $1+g_{1} \geq f(k) \geq f\left(k_{0}\right) \geq f(3)=4 d_{1}-6$. Q.E.D.

Definition 5. Let $G$ be a semi-simple algebraic group, and let $\rho: G$ $\rightarrow G L(V(n))$ be an irreducible $n$-dimensional representation of $G$. Then a triplet $\left(G \times G L(m), \rho \otimes \Lambda_{1}, V(n) \otimes V(m)\right)$ is an irreducible P.V. for $n \leq m$, because the natural action of $G L(m)$ on $V(n) \oplus \cdots \oplus V(n)$ ( $m$ copies) for $m \geq n$, yields a P.V. This triplet is called a trivial P.V. It is reduced except for $G=S L(n)$ with $m>n>m / 2, \rho=\Lambda_{1}$. When $G=S L(n)$ with $m>n>m / 2, \rho=\Lambda_{1}$, its castling transform $(S L(m-n)$ $\left.\times G L(m), \Lambda_{1} \otimes \Lambda_{1}, V(m-n) \otimes V(m)\right)$ is a reduced trivial P.V.

From now on, we shall consider case by case according to the type of the Lie algebra $\mathfrak{g}_{1}$ of $G_{1}$.

I) The case for $G_{1} \sim S L(n)$ (i.e., $g_{1}=\mathfrak{g l}(n, C)$ )

We may assume that $G_{1}=S L(n)$. First of all, we shall determine the irreducible representations $\rho: S L(n) \rightarrow G L(V)$ satisfying $\operatorname{dim} V \leq n^{2}$. We denote $\rho$ by $\Lambda$ when the highest weight of the infinitesimal represen- 
tation $d \rho$ of $\rho$ is $\Lambda$. Let $\Lambda_{1}, \cdots, \Lambda_{n-1}$ be the fundamental dominant weights (see Example 21 in $\S 1$ and let $A=\sum_{i=1}^{n-1} m_{i} \Lambda_{i}\left(m_{i} \geq 0\right.$, integer $1 \leq i \leq n$ -1) be a dominant integral form satisfying $d(\Lambda) \leq n^{2}$.

LEMMA 6. (1) $1 \leq m_{1} \leq 3(n=2)$.

(2) $0 \leq m_{1}+m_{n-1} \leq 2(n \geq 3)$.

(3) $m_{2}, m_{n-2}=0$ or 1 , and if $m_{2}=1$ (resp. $m_{n-2}=1$ ), then $m_{n-2}=0$ (resp. $m_{2}=0$ ) and $m_{1}=m_{n-1}=0(n \geq 4)$.

(4) $m_{3}, m_{n-3}=0$ or 1 , and if $m_{3}=1$ or $m_{n-3}=1$, then $m_{j}=0$ for all other $j(8 \geq n \geq 6)$.

(5) $m_{4}=0(n=8)$.

(6) $m_{j}=0$ for $3 \leq j \leq n-3(n \geq 9)$.

Proof. To prove this lemma, we shall use Corollary 13 in $\S 1$ and the results of Example 24 in $\S 1$.

(1) When $n=2$, we have $\Lambda=m_{1} \Lambda_{1}$ and $d\left(m_{1} \Lambda_{1}\right)=m_{1}+1$. Hence $d(\Lambda)=m_{1}+1 \leq 4=n^{2}$ if and only if $m_{1} \leq 3$. Since $\Lambda \neq 0$, we have $1 \leq m_{1}$.

(2) Since $d\left(3 \Lambda_{1}\right)=d\left(3 \Lambda_{n-1}\right)=\frac{1}{6} n(n+1)(n+2)>n^{2}$ and $d\left(2 \Lambda_{1}+\Lambda_{n-1}\right)$ $=d\left(\Lambda_{1}+2 \Lambda_{n-1}\right)=\frac{1}{2} n(n-1)(n+2)>n^{2}$ if $n \geq 3$, we have $0 \leq m_{1}, m_{n-1}$ $\leq 2$ and $m_{n-1}=0$ (resp. $m_{1}=0$ ) if $m_{1}=2$ (resp. $m_{n-1}=2$ ), i.e., $0 \leq m_{1}$ $+m_{2} \leq 2$ in view of Corollary 13 in $\S 1$.

(3) Since $d\left(2 \Lambda_{2}\right)=d\left(2 \Lambda_{n-2}\right)=\frac{1}{12} n^{2}\left(n^{2}-1\right)>n^{2}$ if $n \geq 4$, we have $m_{2}$, $m_{n-2}=0$ or 1 . Since $d\left(\Lambda_{1}+\Lambda_{2}\right)=d\left(\Lambda_{n-1}+\Lambda_{n-2}\right)=\frac{1}{3} n\left(n^{2}-1\right)>n^{2}, d\left(\Lambda_{1}\right.$ $\left.\Lambda_{n-2}\right)=d\left(\Lambda_{n-1}+\Lambda_{2}\right)=\frac{1}{2} n(n+1)(n-2)>n^{2}$, and $d\left(\Lambda_{2}+\Lambda_{n-2}\right)=\frac{1}{4} n^{2}(n+$ 1) $(n-3)>n^{2}$ if $n \geq 4, m_{2}=1$ (resp. $m_{n-2}=1$ ) implies that $m_{n-2}=0$ (resp. $m_{2}=0$ ) and $m_{1}=m_{n-1}=0$ in view of Corollary 13 in $\S 1$.

(4) It is sufficient to show that $d\left(\Lambda_{3}+\Lambda_{\nu}\right)>n^{2}$ for $1 \leq \nu \leq n-1$, $6 \leq n \leq 8$. For $\nu=1,2$, we have $d\left(\Lambda_{3}+\Lambda_{1}\right)=\frac{1}{8} n\left(n^{2}-1\right)(n-2)>n^{2}$ and $d\left(\Lambda_{3}+\Lambda_{2}\right)=\frac{1}{24} n^{2}\left(n^{2}-1\right)(n-2)>n^{2}(6 \leq n \leq 8)$. Since $d\left(\Lambda_{3}+\Lambda_{\nu+1}\right) / d\left(\Lambda_{3}\right.$ $\left.+\Lambda_{\nu}\right)=(\nu-1)(n-\nu) /\left(\nu^{2}-4\right) \geq 1$ for $3 \leq \nu \leq \frac{1}{4}\left(n+1+\sqrt{\left.n^{2}-6 n+33\right)}\right.$, we have $d\left(\Lambda_{3}+\Lambda_{\nu}\right) \geq d\left(2 \Lambda_{3}\right)=\frac{1}{144} n^{2}(n-1)^{2}(n+1)(n-2)>n^{2}$ for $4 \leq \nu \leq$ $\frac{1}{4}\left(n+5+\sqrt{n^{2}-6 n+33}\right)$. Similarly if $\frac{1}{4}\left(n+5+\sqrt{n^{2}-6 n+33}\right)<\nu \leq$ $n-1$, we have $d\left(\Lambda_{3}+\Lambda_{\nu}\right) \geq d\left(\Lambda_{3}+\Lambda_{n-1}\right)=\frac{1}{6} n\left(n^{2}-1\right)(n-3)>n^{2}$ (see Example 24, (4) in $\S 1$ ).

(5) Since $d\left(\Lambda_{4}\right)=\left(\begin{array}{l}8 \\ 4\end{array}\right)=70>64=n^{2}$, we have $m_{4}=0(n=8)$.

(6) Since $d\left(\Lambda_{j}\right)=\left(\begin{array}{l}n \\ j\end{array}\right) \geq\left(\begin{array}{l}n \\ 3\end{array}\right)=d\left(\Lambda_{3}\right)=\frac{1}{6} n(n-1)(n-2)>n^{2}$, we have $m_{j}=0$ for $3 \leq j \leq n-3, n \geq 9$.

Q.E.D. 
Proposition 7. Let $\rho: S L(n) \rightarrow G L(V)$ be an irreducible representation satisfying $\operatorname{dim} V \leq n^{2}$. Then,

i) $\rho$ is one of: $\Lambda_{1}, 2 \Lambda_{1}, 3 \Lambda_{1}(n=2)$.

ii) $\rho$ is one of : $\Lambda_{1}, \Lambda_{n-1} ; \Lambda_{2}, \Lambda_{n-2} ; 2 \Lambda_{1}, 2 \Lambda_{n-1} ; \Lambda_{1}+\Lambda_{n-1}$ $(n \geq 9$ or $5 \geq n \geq 3)$.

iii) $\rho$ is one of : $\Lambda_{1}, \Lambda_{n-1} ; \Lambda_{2}, \Lambda_{n-2} ; 2 \Lambda_{1}, 2 \Lambda_{n-1} ; \Lambda_{1}+\Lambda_{n-1} ; \Lambda_{3}, \Lambda_{n-3}$ $(8 \geq n \geq 6)$.

Proof. i) is from (1) in Lemma 6. By (5) and (6) in Lemma 6, we may assume that $\Lambda$ is of the form $\Lambda=m_{1} \Lambda_{1}+m_{2} \Lambda_{2}+m_{3} \Lambda_{3}+m_{n-3} \Lambda_{n-3}$ $+m_{n-2} \Lambda_{n-2}+m_{n-1} \Lambda_{n-1}$. Assume that $6 \leq n \leq 8$. If $m_{3}=1$ (resp. $m_{n-3}$ =1), we have $\Lambda=\Lambda_{3}$ (resp. $\Lambda=\Lambda_{n-3}$ ) by (4). If $m_{3}=m_{n-3}=0$, we have $\Lambda=m_{1} \Lambda_{1}+m_{2} \Lambda_{2}+m_{n-2} \Lambda_{n-2}+m_{n-1} \Lambda_{n-1}$. By (6), $\Lambda$ is always of this form if $n \geq 9$, or $n \leq 5$. Assume that $n \geq 4$. If $m_{2}=1$ (resp. $m_{n-2}=1$ ), we have $\Lambda=\Lambda_{2}$ (resp. $\Lambda=\Lambda_{n-2}$ ) by (3). If $m_{2}=m_{n-2}=0$, we have $\Lambda=$ $m_{1} \Lambda_{1}+m_{n-1} \Lambda_{n-1}$. If $n=3, \Lambda$ is always of this form. Assume that $n \geq 3$. Since $A \neq 0$ and by (2) in Lemma 6 , we have $1 \leq m_{1}+m_{n-1} \leq$ 2, i.e., $\Lambda=\Lambda_{1}, \Lambda_{n-1}, 2 \Lambda_{1}, \Lambda_{1}+\Lambda_{n-1}, 2 \Lambda_{n-1}$.

Q.E.D.

COROLLARY 8. Let $\rho: S L(n) \rightarrow G L(V)$ be an irreducible representation of $S L(n)$ with $n \geq 3$. Assume that $\rho \neq \Lambda_{1}, \Lambda_{n-1}, \Lambda_{2}, \Lambda_{n-2}$. Then if we put $d=\operatorname{dim} V$, we have $d \geq \frac{1}{2} n(n+1)(n \neq 6)$ and $d \geq 20(n=6)$.

Proof. Since $d\left(\Lambda_{1}+\Lambda_{n-1}\right)=n^{2}-1>d\left(2 \Lambda_{1}\right)=d\left(2 \Lambda_{n-1}\right)=\frac{1}{2} n(n+1)$ $(n \geq 3)$, we have $d \geq d\left(2 \Lambda_{1}\right)=\frac{1}{2} n(n+1)$ for $n \geq 9$ or $5 \geq n \geq 3$ by Proposition 7 , ii). Since $d\left(\Lambda_{3}\right)=d\left(\Lambda_{n-3}\right)=\frac{1}{6} n(n-1)(n-2)$, we have $d\left(\Lambda_{3}\right)$ $>d\left(2 \Lambda_{1}\right)$ for $n=7,8$ and $d\left(2 \Lambda_{1}\right)=21>d\left(\Lambda_{3}\right)=20$ for $n=6$. By Proposition 7 , iii), we obtain our assertion.

Q.E.D.

Proposition 9. Let $(G, \rho, V)$ be a reduced triplet with $k=1, G_{1} \sim$ $S L(n)$ satisfying $\operatorname{dim} G \geq \operatorname{dim} V$. Then it is equivalent to one of the following triplets.

(1) $\left(G L(n), \Lambda_{1}, V(n)\right)(n \geq 1)$.

(2) $\left(G L(n), 2 \Lambda_{1}, V\left(\frac{1}{2} n(n+1)\right)\right)(n \geq 2)$.

(3) $\left(G L(n), \Lambda_{2}, V\left(\frac{1}{2} n(n-1)\right)\right)(n \geq 4)$.

(4) $\left(G L(n), \Lambda_{1}+\Lambda_{n-1}, V\left(n^{2}-1\right)\right)(n \geq 3)$.

(5) $\left(G L(n), \Lambda_{3}, V\left(\frac{1}{6} n(n-1)(n-2)\right)\right)(n=6,7,8)$.

(6) $\left(G L(2), 3 \Lambda_{1}, V(4)\right)$. 
Proof. A triplet $(G, \rho, V)$ with $k=1$ is always reduced. Since the contragredient representation $\Lambda^{*}$ of $\Lambda=\sum_{i=1}^{n-1} m_{i} \Lambda_{i}$ is $\Lambda^{*}=\sum_{i=1}^{n-1} m_{n-i} \Lambda_{i}$ for $S L(n)$, and $(G L(n), \Lambda, V) \simeq\left(G L(n), \Lambda^{*}, V^{*}\right)$, we have our assertion from Proposition 7.

Q.E.D.

Now we shall prove the following proposition.

Proposition 10. Let $(G, \rho, V)$ be a reduced triplet with $G_{1} \sim S L(n)$ satisfying $\operatorname{dim} G \geq \operatorname{dim} V . \quad$ Assume that it is not a trivial P.V. Then we have $1 \leq k \leq 3$. Moreover, when $k=3$, it is equivalent to the triplet $\left(S L(n) \times S L(n) \times G L(2), \Lambda_{1} \otimes \Lambda_{1} \otimes \Lambda_{1}, V(n) \otimes V(n) \otimes V(2)\right)$.

Proof. Assume that $d_{1}=n$, i.e., $\rho_{1}=\Lambda_{1}$ or $\Lambda_{n-1}$. Then (3.1) implies that $n^{2}+g_{2}+\cdots+g_{k} \geq n d_{2} \cdots d_{k}$, i.e., $g_{2}+\cdots+g_{k} \geq n\left(d_{2} \cdots d_{k}-\right.$ $n$ ). If $n \geq d_{2} \cdots d_{k}$, then it is a trivial P.V. (see Definition 5), and if $d_{2} \cdots d_{k}>n>\frac{1}{2} d_{2} \cdots d_{k}$, it is not reduced. Therefore we may assume that $\frac{1}{2} d_{2} \cdots d_{k} \geq n \geq d_{2}$. In this case, we have $n\left(d_{2} \cdots d_{k}-n\right)-d_{2}\left(d_{2}\right.$ $\left.\cdots d_{k}-d_{2}\right)=\left(n-d_{2}\right)\left(d_{2} \cdots d_{k}-n-d_{2}\right) \geq\left(n-d_{2}\right)\left(d_{2} \cdots d_{k}-\frac{1}{2} d_{2} \cdots\right.$ $\left.d_{k}-d_{2}\right)=\frac{1}{2} d_{2}\left(n-d_{2}\right)\left(d_{3} \cdots d_{k}-2\right) \geq 0$ for $k \geq 3$. Now assume that $k \geq 4$. Then we have $2^{k-2} \geq k$, and hence $(k-1)\left(d_{2}^{2}-1\right) \geq\left(d_{2}^{2}-1\right)+\cdots$ $+\left(d_{k}^{2}-1\right) \geq g_{2}+\cdots+g_{k} \geq n\left(d_{2} \cdots d_{k}-n\right) \geq d_{2}\left(d_{2} \cdots d_{k}-d_{2}\right) \geq d_{2}^{2}\left(2^{k-2}\right.$ $-1) \geq(k-1) d_{2}^{2}$, i.e., a contradiction. Thus we have $1 \leq k \leq 3$. Assume that $k=3$. Then we have $\left(d_{2}^{2}-1\right)+\left(d_{3}^{2}-1\right) \geq d_{2}\left(d_{2} d_{3}-d_{2}\right)=d_{2}^{2}\left(d_{3}-1\right)$, and hence $d_{3}^{2}-2 \geq d_{2}^{2}\left(d_{3}-2\right) \geq d_{3}^{2}\left(d_{3}-2\right)$, i.e., $d_{3}^{3}-3 d_{3}^{2}+2=\left(d_{3}-1\right)\left(d_{3}^{2}\right.$ $\left.-2 d_{3}-2\right) \leq 0$. Together with $d_{3} \geq 2$, we have $d_{3}=2$, and hence $G_{3}=$ $S L(2), g_{3}=3, \rho_{3}=\Lambda_{1}$ (see Remark 43 in $\S 1$ ). By the assumption that $d_{2}=\frac{1}{2} d_{2} d_{3} \geq n \geq d_{2}$, we have $d_{2}=n$. Since $n^{2}+g_{2}+g_{3}=n^{2}+g_{2}+3 \geq$ $n d_{2} d_{3}=2 n^{2}$, we have $g_{2} \geq n^{2}-3$. Assume that $G_{2} \neq S L(n)$. Then by Proposition 42 in $\S 1$, we have $g_{2} \leq \frac{1}{2} n(n+1)$ and hence $n^{2}-3 \leq \frac{1}{2} n(n+1)$, i.e., $n \leq 3$. Again by Remark 43 in $\S 1$, we have $n=3, G_{2}=S L(2)$ and $\rho_{2}=2 \Lambda_{1}$. In this case, however, the dimension of $G$ is less than that of $V$ since $\operatorname{dim} G=n^{2}+g_{2}+g_{3}=9+3+3=15$ and $\operatorname{dim} V=n d_{2} d_{3}=18$. Thus we have $G_{2}=S L(n), \rho_{2}=\Lambda_{1}, d_{2}=n$, and hence we obtain our assertion when $d_{1}=n$. Now assume that $d_{1} \neq n$, i.e., $\rho_{1} \neq \Lambda_{1}, \Lambda_{n-1}$. Then we have $d_{1} \geq d\left(\Lambda_{2}\right)=\frac{1}{2} n(n-1)(n \geq 4)$ and $d_{1} \geq d\left(2 \Lambda_{1}\right)=\frac{1}{2} n(n+1)(n=$ $2,3)$. Assume that $k \geq 3$. Then by (3.4), we have $n^{2} \geq 4 d_{1}-6 \geq 2 n(n$ $-1)-6(n \geq 4)$, i.e., $n \leq 2$, and $n^{2} \geq 4 d_{1}-6 \geq 2 n(n+1)-6(n=2,3)$, i.e., $4 \geq 6(n=2), 9 \geq 18(n=3)$. This is a contradiction and hence we have $1 \leq k \leq 2$.

Q.E.D. 
Finally, we shall consider the case for $k=2$.

Proposition 11. Let $(G, \rho, V)$ be a reduced triplet with $k=2, G_{1} \sim$ $S L(n)$ satisfying $\operatorname{dim} G \geq \operatorname{dim} V$. Assume that $\rho_{1} \neq \Lambda_{2}, \Lambda_{n-2}$ and it is not a trivial P.V. Then it is equivalent to the triplet $\left(S L(3) \times G L(2), 2 \Lambda_{1} \otimes\right.$ $\left.\Lambda_{1}, V(6) \times V(2)\right)$.

Proof. If $\rho_{1}=\Lambda_{1}$ or $\Lambda_{n-1}$, then it is a trivial P.V., and hence we may assume that $\rho_{1} \neq \Lambda_{1}, \Lambda_{n-1}, \Lambda_{2}, \Lambda_{n-2}$. First we shall consider the case when $d_{1}-2 \geq d_{2} \geq 2$. Then by Proposition 3 , we have $n^{2}=1+g_{1} \geq$ $2 d_{1}-3$, in particular, $n \geq 3$ since $d_{1} \geq 4$. By Corollary 8 , we have $d_{1}$ $\geq \frac{1}{2} n(n+1) \quad(n \neq 6)$ and $d_{1} \geq 20(n=6)$ and hence $n^{2} \geq n(n+1)-3$ $(n \neq 6)$, i.e., $n=3$, and $n^{2} \geq 37$ if $n=6$, i.e., $n \neq 6$. Thus we get $n=$ 3 , and $9=1+g_{1} \geq 2 d_{1}-3$, i.e., $6 \geq d_{1}(\geq 4)$. Since $6 \geq d(\Lambda) \geq 4$ implies $\Lambda=2 \Lambda_{1}$ (or its dual $2 \Lambda_{2}$ ) for $S L(3)$, we have $\rho_{1}=2 \Lambda_{1}, d_{1}=6$. If $G_{2}=$ $S L\left(d_{2}\right)$, we may assume that $3 \geq d_{2} \geq 2$ since it is not reduced in the case of $6>d_{2}>3$. Since $9+\left(d_{2}^{2}-1\right) \geq 6 d_{2}$ with $3 \geq d_{2} \geq 2$ implies that $d_{2}=$ 2 , we have a triplet $\left(S L(3) \times G L(2), 2 \Lambda_{1} \otimes \Lambda_{1}, V(6) \otimes V(2)\right)$. If $G_{2} \neq S L\left(d_{2}\right)$, we have $\frac{1}{2} d_{2}\left(d_{2}+1\right) \geq g_{2} \geq 6 d_{2}-9\left(d_{1}-2=4 \geq d_{2} \geq 2\right)$ by Proposition 42 in $\S 1$ and (3.1), and hence $d_{2}=2$. This implies that $G_{2}=S L\left(d_{2}\right)$ by Remark 43 in $\S 1$, i.e., a contradiction.

Next, we shall consider the case when $d_{2}=d_{1}$ or $d_{2}=d_{1}-1$. Assume that $G_{2}=S L\left(d_{2}\right)$. In this case it is a trivial P.V. if $d_{2}=d_{1}$, and it is not reduced if $d_{2}=d_{1}-1$ since it belongs to the same castling class as a triplet $\left(G L(n), \rho_{1}, V\left(d_{1}\right)\right)$. Assume that $G_{2} \neq S L\left(d_{2}\right)$. Then we have $n^{2}+\frac{1}{2} d_{2}\left(d_{2}+1\right) \geq d_{1} d_{2}$ by Proposition 42 in $\S 1$. Since $d_{2}=d_{1}$ or $d_{2}=$ $d_{1}-1$, we have

$$
n^{2} \geq \frac{1}{2} d_{1}\left(d_{1}-1\right)
$$

On the other hand, by Corollary 8 we have

$$
d_{1} \geq \frac{1}{2} n(n+1)(n \neq 6), \quad d_{1} \geq 20(n=6) .
$$

From (3.5) and (3.6), we have

$$
\begin{gathered}
n^{2} \geq \frac{1}{4} n(n+1)\left\{\frac{1}{2} n(n+1)-1\right\}(n \neq 6) \quad \text { and } \\
36 \geq 190 \quad \text { if } n=6 .
\end{gathered}
$$

Since (3.7) holds only for $n=2$, we have $d_{1}=3$ from (3.5) and (3.6). As we have assumed that $G_{2} \neq S L\left(d_{2}\right)$ with $d_{2}=d_{1}$ or $d_{2}=d_{1}-1$, we 
have $G_{2}=S L(2), d_{2}=3, \rho_{2}=2 \Lambda_{1}$ by Remark 43 in $\S 1$. In this case, however, the dimension of $G$ is less than that of $V$ since $\operatorname{dim} G=1+$ $3+3=7$ and $\operatorname{dim} V=3 \times 3=9$.

Q.E.D.

Proposition 12. Let $(G, \rho, V)$ be a reduced triplet with $k=2, G_{1} \sim$ $S L(n)$ satisfying $\operatorname{dim} G \geq \operatorname{dim} V$. Assume that $\rho_{1}=\Lambda_{2}$ or $\Lambda_{n-2}$ and it is not a trivial P.V. Then it is equivalent to one of the following triplets.

(1) $\left(S L(n) \times G L(2), \Lambda_{2} \otimes \Lambda_{1}, V\left(\frac{1}{2} n(n-1)\right) \otimes V(2)\right)(n \geq 4)$.

(2) $\left(S L(4) \times G L(3), \Lambda_{2} \otimes \Lambda_{1}, V(6) \otimes V(3)\right)$.

(3) $\left(S L(5) \times G L(3), \Lambda_{2} \otimes \Lambda_{1}, V(10) \otimes V(3)\right)$.

(4) $\left(S L(5) \times G L(4), \Lambda_{2} \otimes \Lambda_{1}, V(10) \otimes V(4)\right)$.

(5) $\left(S L(4) \times G L(2), \Lambda_{2} \otimes 2 \Lambda_{1}, V(6) \otimes V(3)\right)$.

(6) $\quad\left(G L(4) \times S p(2), \Lambda_{2} \otimes \Lambda_{1}, V(10) \otimes V(4)\right)$.

(7) $\left(G L(4) \times S p(3), \Lambda_{2} \otimes \Lambda_{1}, V(10) \otimes V(6)\right)$.

Proof. We may assume that $\rho_{1}=\Lambda_{2}$ and $n \geq 4$. First we consider the case when $G_{2}=S L\left(d_{2}\right)$, i.e., $\rho_{2}=\Lambda_{1}$ or its dual. If $d_{2}=2$, then $\operatorname{dim} G$ $=n^{2}+3>\operatorname{dim} V=n(n-1)$ and it is reduced since $d_{1}=\frac{1}{2} n(n-1) \geq 6$, i.e., $d_{1}-d_{2}>d_{2}$. Thus we obtain (1), and we may assume that $d_{2} \geq 3$. If $\frac{1}{2} n(n-1)=d_{2}$, it is a trivial P.V., and if $\frac{1}{2} n(n-1)>d_{2}>\frac{1}{4} n(n-1)$, it is not reduced. Therefore, we may assume

$$
\frac{1}{4} n(n-1) \geq d_{2} \geq 3 .
$$

Thus if $n=4$, we have $d_{2}=3$, i.e., (2). Since (3.1) implies that $n^{2}+d_{2}^{2}$ $-1>\frac{1}{2} n(n-1) d_{2}(n \geq 5)$, together with (3.8) we obtain

$$
\frac{1}{4}\left[n(n-1)-\sqrt{n^{2}(n-1)^{2}-16\left(n^{2}-1\right)}\right] \geq d_{2} \geq 3 \quad(n \geq 5) .
$$

Thus if $n=5$, we have $d_{2}=3$ or 4 , i.e., (3) or (4). If $n \geq 6$, then (3.9) has no solution $d_{2}$. Now we shall assume that $G_{2} \neq S L\left(d_{2}\right)$. In this case we have $d_{2} \geq 3$ by Remark 43 in $\S 1$. By Proposition 42 in $\S 1$ and (3.1), we have $n^{2}+\frac{1}{2} d_{2}\left(d_{2}+1\right) \geq \frac{1}{2} n(n-1) d_{2}$, i.e.,

$$
d_{2}^{2}-\left(n^{2}-n-1\right) d_{2}+2 n^{2} \geq 0 \text {. }
$$

On the other hand, we have

$$
d_{1}=\frac{n(n-1)}{2} \geq d_{2} \geq 3
$$

Now we shall prove that there is no solution $d_{2}$ which satisfies both (3.10) 
and (3.11) if $n \geq 6$. Let $d_{2}$ be a solution of (3.10) satisfying $d_{2} \geq \frac{1}{2}\left(n^{2}-\right.$ $n-1+\sqrt{\left.\left(n^{2}-n-1\right)^{2}-8 n^{2}\right)}$. Then by (3.11), we have $n(n-1) \geq n^{2}-$ $n-1+\sqrt{\left(n^{2}-n-1\right)^{2}-8 n^{2}}$, i.e., $1 \geq \sqrt{\left(n^{2}-n-1\right)^{2}-8 n^{2}}$. Since $n \geq 6$, we have $1 \geq\left(n^{2}-n-1\right)^{2}-8 n^{2} \geq\left(n^{2}-2 n\right)^{2}-8 n^{2}=n^{2}\left\{(n-2)^{2}-8\right\} \geq 8 n^{2}$, i.e., a contradiction. Hence we have

$$
\begin{aligned}
& 3 \leq d_{2} \leq \frac{1}{2} f(n) \\
& \quad \text { where } f(x)=x^{2}-x-1-\sqrt{\left(x^{2}-x-1\right)^{2}-8 x^{2}} .
\end{aligned}
$$

Since $\frac{1}{2} f(6)<3$, it is sufficient to show that $f(x)(x \geq 6)$ is monotone decreasing, i.e.,

$$
\frac{\partial f}{\partial x}(x) \leq 0 \quad(x \geq 6)
$$

Since

$$
\begin{aligned}
\frac{\partial f}{\partial x}(x) & \cdot \sqrt{\left(x^{2}-x-1\right)^{2}-8 x^{2}} \\
= & (2 x-1) \sqrt{\left(x^{2}-x-1\right)^{2}-8 x^{2}} \\
& -(2 x-1)\left(x^{2}-x-1\right)+8 x \leq 0
\end{aligned}
$$

if and only if $2 x^{2}+3 x+2 \geq 0(x \geq 6)$, we have $(\partial f / \partial x)(x) \leq 0$, and hence $n \leq 5$. If $n=5$, we get $d_{2}=3$ from (3.10) and (3.11). Since $G_{2} \neq S L\left(d_{2}\right)$, we have $g_{2}=3$ by Remark 43 in $\S 1$. In this case, however, we have $\operatorname{dim} G=28<\operatorname{dim} V=30$. Finally, assume that $n=4$. Then from (3.1), (3.11) and Proposition 42 in $\S 1$, we have

$$
\frac{1}{2} d_{2}\left(d_{2}+1\right) \geq g_{2} \geq 6 d_{2}-16 \quad\left(3 \leq d_{2} \leq 6\right) .
$$

If $d_{2}=3$, we have $6 \geq g_{2} \geq 2$, and hence by Remark 43 in $\S 1, G_{2}=$ $S L(2), g_{2}=3$, i.e., (5).

If $d_{2}=4$, then $10 \geq g_{2} \geq 8$. Note that in the case of $d_{2}=4, G_{2}$ must be one of $S L(2), S L(4), S p(2)$, and hence we have $G_{2}=S p(2), g_{2}=10$, i.e., (6).

If $d_{2}=5$, then $15 \geq g_{2} \geq 14$ by (3.13). Note that $d_{2}=5$ only if $G_{2}$ $=S L(2), S L(5)$ and $S p(2)$. Hence we have $d_{2} \neq 5$.

If $d_{2}=6$, then we have $21 \geq g_{2} \geq 20$. Note that $d_{2}=6$ only if $G_{2}$ $=S L(2), S L(3), S L(4), S L(6)$ and $S p(3)$. Hence we have $G_{2}=S p(3), g_{2}=$ 21 , i.e., (7).

Q.E.D.

II) The case for $G_{1} \sim S p(n)$

We may assume that $G_{1}=S p(n)$ and $n \geq 2$ since $S p(1) \simeq S L(2)$ (see 
Example 45 in $\S 1$ ). First of all, we shall consider the irreducible representations $\rho: S p(n) \rightarrow G L(V)$ satisfying $\operatorname{dim} V \leq 1+\operatorname{dim} S p(n)=n(2 n+1)$ +1 . Let $\Lambda_{1}, \cdots, \Lambda_{n}$ be the fundamental dominant weights of $S p(n)$, and let $\Lambda=\sum_{i=1}^{n} m_{i} \Lambda_{i}$ be a dominant integral form satisfying $d(\Lambda) \leq n(2 n+$ 1) +1 .

LEMMA 13. (1) $0 \leq m_{1} \leq 2(n \geq 2)$.

(2) $m_{2}=0$ or 1 , and if $m_{2}=1$, then $m_{1}=0(n \geq 2)$.

(3) $m_{3}=0$ or 1 , and if $m_{3}=1$, then $m_{1}=m_{2}=0(n=3)$.

(4) $m_{\nu}=0$ for $3 \leq \nu \leq n(n \geq 4)$.

Proof. (1): Since $d\left(3 \Lambda_{1}\right)=\frac{2}{3} n(n+1)(2 n+1)>n(2 n+1)+1(n \geq 2)$, we obtain (1) in view of Corollary 13 in $\S 1$ (see (1) in Example 26 in $\S 1$ ). (2): Since $d\left(2 \Lambda_{2}\right)=\frac{1}{3} n(n-1)(2 n-1)(2 n+3)>n(2 n+1)(n \geq 2)$, we have $0 \leq m_{2} \leq 1$, and by $d\left(\Lambda_{1}+\Lambda_{2}\right)=\frac{8}{3} n\left(n^{2}-1\right)>n(2 n+1)+1(n \geq 2)$, we obtain (2) (see (2), (3) in Example 26). (3): Since $d\left(2 \Lambda_{3}\right)=84, d\left(\Lambda_{1}+\Lambda_{3}\right)$ $=70, d\left(\Lambda_{2}+\Lambda_{3}\right)=126$, we have $d\left(\Lambda_{3}+\Lambda_{\nu}\right)>\operatorname{dim} S p(3)+1=22(1 \leq \nu \leq$ $3, n=3$ ) and hence we obtain (3) (see Example 26). Note that $d\left(\Lambda_{3}\right)=$ $14<22=\operatorname{dim} S p(3)+1(n=3)$. (4): Since $d\left(\Lambda_{\nu}\right)=\left(\begin{array}{c}2 n \\ \nu\end{array}\right)-\left(\begin{array}{c}2 n \\ \nu-2\end{array}\right)$, we have $d\left(\Lambda_{3}\right)=\frac{2}{3} n(n-2)(2 n+1)>n(2 n+1)+1(n \geq 4)$. Next we shall show that $d\left(\Lambda_{n}\right)=[2(2 n+1) ! / n !(n+2) !]>n(2 n+1)+1(n \geq 4)$. Put $c_{n}=$ $2 n(n+1) / d\left(\Lambda_{n}\right)=n(n+1) !(n+2) ! /(2 n+1) !$. Since $c_{4}<1$ and $c_{n+1} / c_{n}$ $=(n+2)(n+3) / 2 n(2 n+3)<1(n \geq 2)$, we have $c_{n}<1$ and hence $d\left(\Lambda_{n}\right)$ $>n(2 n+1)$ for $n \geq 4$. Finally, we shall show that $d\left(\Lambda_{\nu}\right) \geq \min \left(d\left(\Lambda_{3}\right)\right.$, $d\left(\Lambda_{n}\right)$ ) for $3 \leq \nu \leq n(n \geq 4)$. Since $d\left(\Lambda_{\nu}\right)-d\left(\Lambda_{\nu-1}\right)=2(2 n+1) !(2 n-2 \nu$ $+3+\sqrt{2 n+3})\left\{\frac{1}{2}(2 n+3-\sqrt{2 n+3})-\nu\right\} / \nu !(2 n-\nu+3) !, d\left(\Lambda_{\nu}\right)$ is monotone increasing if $1 \leq \nu \leq \frac{1}{2}(2 n+3-\sqrt{2 n+3})$ and monotone decreasing if $\frac{1}{2}(2 n+3-\sqrt{2 n+3}) \leq \nu \leq n$. This shows that $d\left(\Lambda_{\nu}\right) \geq \min \left(d\left(\Lambda_{3}\right),\left(d\left(\Lambda_{\nu}\right)\right)\right.$ $(3 \leq \nu \leq n)$ and hence $d\left(\Lambda_{\nu}\right)>n(2 n+1)+1$. In view of Corollary 13 in $\S 1$, we obtain our assertion.

Q.E.D.

Proposition 14. Let $(G, \rho, V)$ be a reduced triplet with $k=1$ and $G_{1} \sim S p(n)$ satisfying $\operatorname{dim} V \leq \operatorname{dim} G$. Then it is equivalent to one of the following triplets.

(1) $\quad\left(G L(1) \times S p(n), \square \otimes \Lambda_{1}, V(1) \otimes V(2 n)\right)$.

(2) $\left(G L(1) \times S p(n), \square \otimes \Lambda_{2}, V(1) \otimes V((n-1)(2 n+1))\right)$.

(3) $\quad\left(G L(1) \times S p(n), \square \otimes 2 \Lambda_{1}, V(1) \otimes V(n(2 n+1))\right)$.

(4) $\quad\left(G L(1) \times S p(3), \square \otimes \Lambda_{3}, V(1) \otimes V(14)\right)$. 
Proof. Since $\operatorname{dim} G=n(2 n+1)+1$, we have $d(\Lambda) \leq n(2 n+1)+1$ for $\rho_{1}=\Lambda$. By Lemma 13, $\Lambda$ must be one of $\Lambda_{1}, 2 \Lambda_{1}, \Lambda_{2}(n \geq 2)$ and $\Lambda_{3}$ $(n=3)$. Since a triplet with $k=1$ is always reduced, we have our assertion.

Q.E.D.

Proposition 15. Let $(G, \rho, V)$ be a reduced triplet with $G_{1} \sim S p(n)$ satisfying $\operatorname{dim} V \leq \operatorname{dim} G$. Assume that $\rho_{1} \neq \Lambda_{1}$. Then we have $1 \leq k \leq 2$. Moreover, if $k=2$, it is equivalent to one of the following triplets.

(1) $\left(S p(2) \times G L(2), \Lambda_{2} \otimes \Lambda_{1}, V(5) \otimes V(2)\right)$.

(2) $\quad\left(S p(2) \times G L(3), \Lambda_{2} \otimes \Lambda_{1}, V(5) \otimes V(3)\right)$.

(3) $\quad\left(G L(1) \times S p(2) \times S p(2), \square \otimes \Lambda_{2} \otimes \Lambda_{1}, V(1) \otimes V(5) \otimes V(4)\right)$.

Proof. Assume that $k \geq 3$. Since $d\left(2 \Lambda_{1}\right)>d\left(\Lambda_{2}\right)$, we have $d_{1}=d(\Lambda) \geq$ $d\left(\Lambda_{2}\right)=(n-1)(2 n+1)$ for $\Lambda \neq \Lambda_{1}$ in view of Proposition 14 . Then together with (3.4), we have $1+n(2 n+1) \geq 4(n-1)(2 n+1)-6$, i.e., $(6 n-11)(n+1) \leq 0$. This is a contradiction since $n \geq 2$, and hence we have $1 \leq k \leq 2$. Now assume that $k=2$. If $d_{1}-2 \geq d_{2} \geq 2$, we have $1+n(2 n+1) \geq 2 d_{1}-3 \geq 2(n-1)(2 n+1)-3$ by Proposition 3. This implies that $n=2$ and $7 \geq d_{1} \geq 5$. Thus we have $\rho_{1}=\Lambda_{2}, d_{1}=5$, and hence $d_{1}-2=3 \geq d_{2} \geq 2$. By Remark 43 in $\S 1$, we obtain (1), (2) and a triplet $\left(S p(2) \times G L(2), \Lambda_{2} \otimes \Lambda_{2}, V(5) \otimes V(3)\right)$. This latter triplet, however, does not satisfy $\operatorname{dim} V \leq \operatorname{dim} G$ since $\operatorname{dim} V=15$ and $\operatorname{dim} G=14$. Assume that $d_{2}=d_{1}$ or $d_{1}-1$. In this case we have $d_{1} d_{2}-\frac{1}{2} d_{2}\left(d_{2}+1\right)=$ $\frac{1}{2} d_{1}\left(d_{1}-1\right)$. Therefore, if $G_{2} \neq S L\left(d_{2}\right)$, together with Proposition 42 in $\S 1$ and (3.1), we have $1+n(2 n+1) \geq \frac{1}{2} d_{1}\left(d_{1}-1\right) \geq \frac{1}{2}(n-1)(2 n+1)\{(n-$ $1)(2 n+1)-1\}$, and hence $n=2, d_{1}=5$, i.e., $\rho_{1}=\Lambda_{2}$. If $d_{2}=d_{1}=5$, then $G_{2}$ must be $S L(2)$ or $S p(2) \quad\left(G_{2} \neq S L(5)\right.$ by assumption). However, in both cases we have $\operatorname{dim} G<\operatorname{dim} V$. If $d_{2}=d_{1}-1=4$, then $G_{2}$ is again $S L(2)$ or $S p(2)$. If $G_{2}=S L(2)$, we have $\operatorname{dim} G<\operatorname{dim} V$, and if $G_{2}$ $=S p(2)$, we obtain (3). Finally, if $G_{2}=S L\left(d_{2}\right)$, it is a trivial P.V. when $d_{1}=d_{2}$, and it is not reduced when $d_{2}=d_{1}-1$.

Q.E.D.

We shall consider the case of $\rho_{1}=\Lambda_{1}$.

LEMMA 16. Let $(G, \rho, V)$ be a triplet with $G_{1} \neq S L\left(d_{1}\right)$ satisfying $\operatorname{dim} V \leq \operatorname{dim} G$. Then we have $d_{1} \geq d_{2} \cdots d_{k}$.

Proof. Assume that $d_{2} \cdots d_{k}>d_{1} \geq d_{2}$ and hence $k \geq 3$. By Proposition 42 in $\S 1$ and (3.1) we have $1+\frac{1}{2} d_{1}\left(d_{1}+1\right)+g_{2}+\cdots+g_{k} \geq d_{1} d_{2}$ $\cdots d_{k}$, i.e., $g_{2}+\cdots+g_{k} \geq \frac{1}{2} d_{1}\left(2 d_{2} \cdots d_{k}-1-d_{1}\right)-1=\frac{1}{2} d_{1}\left(A-d_{1}\right)-1$ 
where $A=2 d_{2} \cdots d_{k}-1$. Since $A / 2>d_{1} \geq d_{2}$ and $x(A-x)$ is a monotone increasing function for $x \leq A / 2$, we have $d_{1}\left(A-d_{1}\right) \geq d_{2}\left(A-d_{2}\right)$. Assume that $k \geq 4$. Then we have $2^{k-2} \geq k$, and hence $(k-1)\left(d_{2}^{2}-1\right)$ $\geq\left(d_{2}^{2}-1\right)+\cdots+\left(d_{k}^{2}-1\right) \geq g_{2}+\cdots+g_{k} \geq \frac{1}{2} d_{1}\left(A-d_{1}\right)-1 \geq \frac{1}{2} d_{2}(A-$ $\left.d_{2}\right)-1=d_{2}^{2}\left(d_{3} \cdots d_{k}-1\right)+\frac{1}{2} d_{2}\left(d_{2}-1\right)-1 \geq d_{2}^{2}\left(2^{k-2}-1\right) \geq(k-1) d_{2}^{2}$. This is a contradiction, and hence we have $k=3$. In this case we have $d_{3}=2$ since $2\left(d_{2}^{2}-1\right) \geq d_{2}^{2}\left(d_{3}-1\right)+\frac{1}{2} d_{2}\left(d_{2}-1\right)-1$ and $d_{3} \geq 3$ implies 0 $\geq \frac{1}{2} d_{2}\left(d_{2}-1\right)+1$. Therefore, we have $\left(d_{2}^{2}-1\right)+\left(2^{2}-1\right) \geq d_{2}^{2}(2-1)+$ $\frac{1}{2} d_{2}\left(d_{2}-1\right)-1$, i.e., $d_{2}\left(d_{2}-1\right) \leq 6$. This implies $d_{2}=2$ or $d_{2}=3$. Вy Remark 43 in $\S 1$ and $G_{1} \neq S L\left(d_{1}\right)$, we have $d_{1} \geq 3$, and $d_{1}=3$ implies $g_{1}=3$. Hence if $d_{2}=2$, then $d_{2} d_{3}=4>d_{1} \geq 2$ and we have $d_{1}=3, g_{1}$ $=3$. In this case, however, we have $10=1+g_{1}+g_{2}+g_{3} \geq d_{1} d_{2} d_{3}=12$, i.e., a contradiction. Hence we have $d_{2}=3$, and $d_{2} d_{3}=6>d_{1} \geq d_{2}=3$. Assume that $d_{1}=3$. Then $15=1+3+8+3 \geq 1+g_{1}+g_{2}+g_{3} \geq d_{1} d_{2} d_{3}$ $=18$, i.e., a contradiction. Since $d_{1}=4$ or 5 does not satisfy the inequality $1+d_{1}\left(d_{1}+1\right) / 2+8+3 \geq 1+g_{1}+g_{2}+g_{3} \geq d_{1} d_{2} d_{3}=6 d_{1}$, there is no triplet such as $G_{1} \neq S L\left(d_{1}\right), \operatorname{dim} V \leq \operatorname{dim} G$ and $d_{2} \leq d_{1}<d_{2} \cdots d_{k}$. Hence we have $d_{1} \geq d_{2} \cdots d_{k}$.

Q.E.D.

Lemma 17. Let $(G, \rho, V)$ be a triplet satisfying $\operatorname{dim} G \geq \operatorname{dim} \Lambda^{2}(V)=$ $\frac{1}{2} d(d-1)$ where $d=\operatorname{dim} V$. Then it is equivalent to one of the following triplets.

(1) $\left(G L(d), \Lambda_{1}, V(d)\right)$.

(2) $\left(G L(1) \times S p(m), \square \otimes \Lambda_{1}, V(1) \otimes V(d)\right)(d=2 m)$.

(3) $\left(G L(1) \times S O(d), \square \otimes \Lambda_{1}, V(1) \otimes V(d)\right)$.

Moreover, if $\operatorname{dim} G \geq S^{2}(V)=\frac{1}{2} d(d+1)$, it is equivalent to (1) or (2).

Proof. First assume that $k \geq 2$. In this case, we may assume that $G=\tilde{G}_{1} \times \tilde{G}_{2}, V=V\left(n_{1}\right) \otimes V\left(n_{2}\right), d=n_{1} n_{2}, n_{1} \geq n_{2} \geq 2$, where $\tilde{G}_{1}$ and $\tilde{G}_{2}$ are not necessarily simple. If $n_{2} \geq 3$, then we have $2 n_{1}^{2}-1 \geq 1+\left(n_{1}^{2}-1\right)$ $+\left(n_{2}^{2}-1\right) \geq \operatorname{dim} G \geq \frac{1}{2} d(d-1)=\frac{1}{2} n_{1} n_{2}\left(n_{1} n_{2}-1\right) \geq \frac{9}{2} n_{1}^{2}-\frac{3}{2} n_{1} \geq 3 n_{1}^{2}$. This is a contradiction, and hence we have $n_{2}=2$. Then we have $n_{1}=2$ since $n_{1}^{2}+3 \geq \operatorname{dim} G \geq \frac{1}{2} d(d-1)=2 n_{1}^{2}-n_{1}$, i.e., $n_{1}\left(n_{1}-1\right) \leq 3$. In this case we have $\left(S L(2) \times G L(2), \Lambda_{1} \otimes \Lambda_{1}, V(2) \otimes V(2)\right) \simeq\left(G L(1) \times S O(4), \square \otimes \Lambda_{1}, V(1)\right.$ $\otimes V(4)$ ) (see Example 51 in $\S 1$ ), i.e., (3) for $d=4$. Next, assume that $k=1$. Since we have seen in $\S 1$ that the least representation degree $d$ of $\left(G_{2}\right)$ (resp. $F_{4}, E_{6}, E_{7}, E_{8}$ ) is 7 (resp. 26, 27, 56, 248) while the dimension $g$ of $\left(G_{2}\right)$ (resp. $\left.F_{4}, E_{6}, E_{7}, E_{8}\right)$ is 14 (resp. 52,78, 133, 248), we have $g<$ 
$\frac{1}{2} d(d-1)$ and hence $G_{1}$ is not an exceptional algebraic group. If $G_{1}=$ $S L(n)$, i.e., $G=G L(n)$ we have $\rho=\Lambda_{1}(n \geq 1)$, i.e., (1), $\rho=2 \Lambda_{1}(n=2)$, and $\rho=\Lambda_{2}(n=4)$ in view of Proposition 9. Note that $\left(G L(2), 2 \Lambda_{1}, V(3)\right)$ $\simeq\left(G L(1) \times S O(3), \square \otimes \Lambda_{1}, V(1) \otimes V(3)\right)$, i.e., (3) for $d=3$, and $(G L(4)$, $\left.\Lambda_{2}, \quad V(6)\right) \simeq\left(G L(1) \times S O(6), \square \otimes \Lambda_{1}, V(1) \otimes V(6)\right)$, i.e., (3) for $d=6$ (see Example 45 in $\S 1$ ). If $G_{1}=S p(n)$, we have $\rho_{1}=\Lambda_{1}$, i.e., (2) and $\rho_{1}=\Lambda_{2}(n$ $=2$ ) in view of Proposition 14. Note that $\left(G L(1) \times S p(2), \square \otimes \Lambda_{2}, V(1)\right.$ $\otimes V(5)) \simeq\left(G L(1) \times S O(5), \square \otimes \Lambda_{1}, V(1) \otimes V(5)\right)$, i.e., (3) for $d=5$. If $G_{1}$ $=S O(n), \rho_{1}=\Lambda_{1}$, i.e., (3) since in this case $\operatorname{dim} G=1+\frac{1}{2} n(n-1) \geq \frac{1}{2} d(d$ -1 ), i.e., $d=n$. The second assertion is now obvious.

Q.E.D.

Proposition 18. Let $(G, \rho, V)$ be a reduced triplet with $k \geq 2, G_{1} \sim$ $S p(n), \rho_{1}=\Lambda_{1}$. Then it is not a P.V. unless it is a trivial P.V. or equivalent to one of the following triplets.

(1) $\left(S p(n) \times G L(m), \Lambda_{1} \otimes \Lambda_{1}, V(2 n) \otimes V(m)\right)(n \geq m \geq 2)$.

(2) $\quad\left(G L(1) \times S p(n) \times S p(m), \square \otimes \Lambda_{1} \otimes \Lambda_{1}, V(1) \otimes V(2 n) \otimes V(2 m)\right)(n \geq$ $m \geq 2)$.

(3) $\quad\left(G L(1) \times S p(n) \times S O(m), \square \otimes \Lambda_{1} \otimes \Lambda_{1}, V(1) \otimes V(2 n) \otimes V(m)\right)(2 n \geq$ $m \geq 3)$.

Proof. From Proposition 13 in §2, Lemma 16 and Lemma 17, our assertion is obvious.

Q.E.D.

III. The case for $G_{1} \sim S O(n)$

Since $S O(n)$ is not simply connected, we have to consider its covering group $\operatorname{Spin}(n)$ when $\rho_{1}$ is a (half-) spin representation. However, we need not consider $\operatorname{Spin}(8)$ since $\left(S O(8), \Lambda_{1}, V(8)\right) \simeq(\operatorname{Spin}(8)$, half-spin rep. $V(8)$ ) (see Example 28 in $\S 1$ ). Note that if $\Lambda_{m-1}$ and $\Lambda_{m}$ are two inequivalent half-spin representations of $\operatorname{Spin}(2 m)$, we have $\left(\operatorname{Spin}(2 m), \Lambda_{m-1}\right.$, $\left.V\left(2^{m-1}\right)\right) \simeq\left(\operatorname{Spin}(2 m), \Lambda_{m}, V\left(2^{m-1}\right)\right)$. We denote it by $(\operatorname{Spin}(2 m)$, half-spin rep. $\left.V\left(2^{m-1}\right)\right)$. Since we have seen in Example 45 in $\S 1$ that $\operatorname{Spin}(6) \simeq$ $S L(4), \operatorname{Spin}(5) \simeq S p(2), S p i n(4) \simeq S L(2) \times S L(2), \operatorname{Spin}(3) \simeq S L(2)$, we may assume that $n \geq 7$.

Proposition 19. Let $(G, \rho, V)$ be a reduced triplet with $G_{1} \sim S O(n)$, $\rho_{1}=\Lambda_{1}$ Then it is not a P.V. unless it is a trivial P.'V. or equivalent to one of the following triplets.

(1) $\left(S O(n) \times G L(m), \Lambda_{1} \otimes \Lambda_{1}, V(n) \otimes V(m)\right)(n \geq 7, n / 2 \geq m \geq 1)$.

(2) $\quad\left(G L(1) \times S O(n) \times S p(m), \square \otimes \Lambda_{1} \otimes \Lambda_{1}, V(1) \otimes V(n) \otimes V(2 m)\right)$ $(n \geq 7, n \geq 2 m \geq 4)$. 
Proof. From Proposition 14 in $\S 2$, Lemma 16 and Lemma 17, our assertion is obvious.

Q.E.D.

Proposition 20. Let $(G, \rho, V)$ be a reduced triplet with $G_{1} \sim S O(n)$, $\rho_{1} \neq \Lambda_{1}$, satisfying $\operatorname{dim} V \leq \operatorname{dim} G$. Then we have $1 \leq k \leq 2$. Moreover, if $k=1$, it is equivalent to one of the following triplets.

(1) $(G L(1) \times \operatorname{Spin}(7), \square \otimes \operatorname{spin}$ rep., $V(1) \otimes V(8))$.

(2) $\quad(G L(1) \times S p i n(9) . \square \otimes \operatorname{spin} r e p ., V(1) \otimes V(16))$.

(3) $(G L(1) \times \operatorname{Spin}(10), \square \otimes$ half-spin rep., $V(1) \otimes V(16))$.

(4) $(G L(1) \times \operatorname{Spin}(11), \square \otimes \operatorname{spin}$ rep., $V(1) \otimes V(32))$.

(5) $(G L(1) \times \operatorname{Spin}(12), \square \otimes$ half-spin rep., $V(1) \otimes V(32))$.

(6) $(G L(1) \times \operatorname{Spin}(13), \square \otimes \operatorname{spin}$ rep., $V(1) \otimes V(64))$.

(7) $(G L(1) \times \operatorname{Spin}(14), \square \otimes$ half-spin rep., $V(1) \otimes V(64))$.

(8) $\left(G L(1) \times S O(n), \square \otimes \Lambda_{2}, V(1) \otimes V\left(\frac{1}{2} n(n-1)\right)(n \geq 7)\right.$.

Proof. First we shall show that $d_{1} \geq \frac{1}{2} n(n-1)(n \geq 15)$ and $d_{1} \geq$ $2^{[(n-1) / 2]}(14 \geq n \geq 7)$ where $[\alpha]$ is an integer satisfying $1>\alpha-[\alpha] \geq 0$. As we have seen in Example 27 and Example 28 in $\S 1, d\left(\Lambda_{\nu}\right)=\left(\begin{array}{l}n \\ \nu\end{array}\right)$ for $1 \leq \nu \leq[(n-3) / 2]$ and $d\left(\Lambda_{\nu}\right)=2^{[(n-1) / 2]}$ for $\nu=(n-1) / 2$ when $n$ is odd, and for $\nu=n / 2, n / 2-1$, when $n$ is even. Since $d\left(2 \Lambda_{1}\right)=\frac{1}{2}(n-1)(n+2)$ $>d\left(\Lambda_{2}\right)=\frac{1}{2} n(n-1)$, we have $d_{1}=d(\Lambda) \geq \min \left\{\frac{1}{2} n(n-1), 2^{[(n-1) / 2]}\right\}$ for any $\Lambda \neq \Lambda_{1}$. Since $2^{[(n-1) / 2]}>\frac{1}{2} n(n-1)(n \geq 15)$ and $\frac{1}{2} n(n-1)>2^{[(n-1) / 2]}(14 \geq$ $n \geq 7)$, we have $d_{1} \geq \frac{1}{2} n(n-1) \quad(n \geq 15)$ and $d_{1} \geq 2^{[(n-1) / 2]}(14 \geq n \geq 7)$. Here if $n=8$, we may assume that $d_{1} \geq d\left(\Lambda_{2}\right)=28$ since $\left(S O(8), \Lambda_{1}, V(8)\right.$ ) $\simeq(\operatorname{Spin}(8)$, half-spin rep., $V(8))$. Assume that $k \geq 3$. Then together with (3.4), if $n \geq 15$ or $n=8$, we have $1+\frac{1}{2} n(n-1) \geq 2 n(n-1)-6$, i.e., $n(n-1) \leq \frac{14}{3}$. This is a contradiction. If $14 \geq n \geq 7$ and $n \neq 8$, we have $1+\frac{1}{2} n(n-1) \geq 4 \cdot 2^{[(n-1) / 2]}-6$. This is also a contradiction and hence we have $1 \leq k \leq 2$. Since $2^{[(n-1) / 2]}>\frac{1}{2} n(n-1)+1(n \geq 15)$, and $d\left(2 \Lambda_{1}\right)>$ $\frac{1}{2} n(n-1)+1(n \geq 7)$, we have $d(\Lambda) \leq \frac{1}{2} n(n-1)+1$ if and only if $\Lambda$ is a (half-) spin representation for $7 \leq n \leq 14, n \neq 8$, or $\Lambda=\Lambda_{2}$. Hence we obtain our assertion.

Q.E.D.

Proposition' 21. Let $(G, \rho, V)$ be a reduced triplet with $k=2, G_{1} \sim$ $S O(n), \quad \rho_{1} \neq \Lambda_{1}$ satisfying $\operatorname{dim} V \leq \operatorname{dim} G$. Then it is a trivial P.V. or equivalent to one of the following triplets.

(1) $\left(\operatorname{Spin}(7) \times G L(d)\right.$, spin rep. $\left.\otimes \Lambda_{1}, V(8) \otimes V(d)\right)(2 \leq d \leq 4)$.

(2) $\left(\operatorname{Spin}(7) \times G L(2)\right.$, spin rep. $\left.\otimes 2 \Lambda_{1}, V(8) \otimes V(3)\right)$. 
(3) $\quad\left(G L(1) \times S p i n(7) \times S p(2), \square \otimes \operatorname{spin} r e p . \otimes \Lambda_{1}, V(1) \otimes V(8) \otimes V(4)\right)$.

(4) $\left(\operatorname{Spin}(9) \times G L(2)\right.$, spin rep. $\left.\otimes \Lambda_{1}, V(16) \otimes V(2)\right)$.

(5) $\left(\operatorname{Spin}(10) \times G L(d)\right.$, half-spin rep. $\left.\otimes \Lambda_{1}, V(16) \otimes V(d)\right)(2 \leq d \leq 3)$.

(6) $\left(\operatorname{Spin}(10) \times G L(2)\right.$, half-spin rep. $\left.\otimes 2 \Lambda_{1}, V(16) \otimes V(3)\right)$.

(7) $\left(\operatorname{Spin}(12) \times G L(2)\right.$, half-spin rep. $\left.\otimes \Lambda_{1}, V(32) \otimes V(2)\right)$.

Proof. First assume that $d_{1}-2 \geq d_{2} \geq 2$. Then by Proposition 3, we have $1+\frac{1}{2} n(n-1) \geq 2 d_{1}-3$. If $\rho_{1}$ is not a (half-) spin representation, by the proof of Proposition 20, we have $d_{1} \geq d\left(\Lambda_{2}\right)=\frac{1}{2} n(n-1)$ and hence $1+\frac{1}{2} n(n-1) \geq n(n-1)-3$, i.e., $n(n-1) \leq 8(n \geq 7)$. This is a contradiction, and hence $\rho_{1}$ is a (half-) spin representation $(n \neq 8)$. In this case we have $1+\frac{1}{2} n(n-1) \geq 2 \cdot 2^{[(n-1) / 2]}-3(n \neq 8, n \geq 7)$ and hence we get $n=7,9$ (spin rep.) and $n=10,12$ (half-spin rep.). We shall consider each case. In the case of $n=7$, we have $2 \leq d_{2} \leq d_{1}-2=6$. If $G_{2}=S L\left(d_{2}\right)$, we have $2 \leq d_{2} \leq 4$, i.e., (1) since otherwise it is not reduced. If $G_{2} \neq S L\left(d_{2}\right)$, by Proposition 42 in $\S 1$ and (3.1), we have $1+21+$ $\frac{1}{2} d_{2}\left(d_{2}+1\right) \geq 8 d_{2}\left(2 \leq d_{2} \leq 6\right)$, hence $d_{2}=3, G_{2}=S L(2)$, i.e., (2) or $d_{2}=$ $4, G_{2}=S p(2), \rho_{2}=\Lambda_{1}$, i.e., (3) since otherwise the condition (3.1) or $G_{2}$ $\neq S L\left(d_{2}\right)$ is not satisfied. In the case of $n=9$, we have $2 \leq d_{2} \leq 14=$ $d_{1}-2$. If $G_{2} \neq S L\left(d_{2}\right)$, we have $g_{2} \leq \frac{1}{2} d_{2}\left(d_{2}+1\right)$ and $d_{2} \geq 3$, and hence (3.1) implies $1+36+\frac{1}{2} d_{2}\left(d_{2}+1\right) \geq 16 d_{2}$, i.e., $\left(\frac{31}{2}-d_{2}\right)^{2} \geq 166+\frac{1}{4}\left(14 \geq d_{2}\right.$ $\geq 3$ ). This is a contradiction and hence we have $G_{2}=S L\left(d_{2}\right)$. In this case we have $2 \leq d_{2} \leq 8$ since otherwise it is not reduced. Then (3.1) implies that $1+36+\left(d_{2}^{2}-1\right) \geq 16 d_{2}\left(2 \leq d_{2} \leq 8\right)$ and hence $d_{2}=2$, i.e., (4). In the case of $n=10$, we have $2 \leq d_{2} \leq 14 \leq d_{1}-2$. If $G_{2}=S L\left(d_{2}\right)$, we have $2 \leq d_{2} \leq 8$ since otherwise it is not reduced. Then (3.1) implies $1+45+\left(d_{2}^{2}-1\right) \geq 16 d_{2}\left(2 \leq d_{2} \leq 8\right)$ and hence $2 \leq d_{2} \leq 3$, i.e., (5). If $G_{2} \neq S L\left(d_{2}\right)$, we have $1+45+\frac{1}{2} d_{2}\left(d_{2}+1\right) \geq 16 d_{2}\left(3 \leq d_{2} \leq 14\right)$ and hence $d_{2}=3, G_{2}=S L(2), \rho_{2}=2 \Lambda_{1}$, i.e., (6). In the case of $n=12$, we have $2 \leq d_{2} \leq 30$. If $G_{2} \neq S L\left(d_{2}\right)$, we have $1+66+\frac{1}{2} d_{2}\left(d_{2}+1\right) \geq 32 d_{2}\left(3 \leq d_{2}\right.$ $\leq 30$ ). This has no solution and hence $G_{2}=S L\left(d_{2}\right)$. In this case we may assume that $2 \leq d_{2} \leq 16$ since otherwise it is not reduced. Then by (3.1) we have $1+66+\left(d_{2}^{2}-1\right) \geq 32 d_{2}\left(2 \leq d_{2} \leq 16\right)$ and hence $d_{2}=2$, i.e., (7). Finally, we shall consider the case when $d_{2}=d_{1}$ or $d_{2}=d_{1}-1$. In this case we have $d_{1} d_{2}-\frac{1}{2} d_{2}\left(d_{2}+1\right)=\frac{1}{2} d_{1}\left(d_{1}-1\right)$. Hence if $G_{2} \neq S L\left(d_{2}\right)$, by (3.1) we have $1+\frac{1}{2} n(n-1) \geq \frac{1}{2} d_{1}\left(d_{1}-1\right)$, i.e., $n=d_{1}, \rho_{1}=\Lambda_{1}$. As our assumption is $\rho_{1} \neq \Lambda_{1}$, we have $G_{2}=S L\left(d_{2}\right)$. However, in this case it is a trivial P.V. if $d_{2}=d_{1}$, and it is not reduced if $d_{2}=d_{1}-1$. Q.E.D. 
IV. The case when $G_{1}$ is an exceptional algebraic group

We shall denote by $\left(G_{2}\right)$ the exceptional simple algebraic group of dimension 14, of rank 2, to distinguish it from the second group $G_{2}$.

Proposition 22. Let $(G, \rho, V)$ be a reduced triplet satisfying $\operatorname{dim} V$ $\leq \operatorname{dim} G$ with an exceptional simple algebraic group $G_{1}$. Then we have $1 \leq k \leq 2$. Moreover, if $k=1$, it is equivalent to one of the following triplets.

(1) $\left(G L(1) \times\left(G_{2}\right), \square \otimes A_{2}, V(1) \otimes V(7)\right)$.

(2) $\left(G L(1) \times\left(G_{2}\right), \square \otimes \Lambda_{1}, V(1) \otimes V(14)\right)$.

(3) $\quad\left(G L(1) \times F_{4}, \square \otimes \Lambda_{4}, V(1) \otimes V(26)\right)$.

(4) $\quad\left(G L(1) \times F_{4}, \square \otimes A_{1}, V(1) \otimes V(52)\right)$.

(5) $\quad\left(G L(1) \times E_{6}, \square \otimes \Lambda_{1}, V(1) \otimes V(27)\right)$.

(6) $\quad\left(G L(1) \times E_{6}, \square \otimes A_{6}, V(1) \otimes V(78)\right)$.

(7) $\quad\left(G L(1) \times E_{7}, \square \otimes A_{6}, V(1) \otimes V(56)\right)$.

(8) $\left(G L(1) \times E_{7}, \square \otimes \Lambda_{1}, V(1) \otimes V(133)\right)$.

(9) $\left(G L(1) \times E_{8}, \square \otimes \Lambda_{7}, V(1) \otimes V(248)\right)$.

Proof. If $k=1$, we obtain our assertion from the calculation of representation degree in $\S 1$ (see Example 30 for $\left(G_{2}\right)$, Example 38 for $F_{4}$, Example 39 for $E_{6}$, Example 40 for $E_{7}$ and Example 41 for $E_{8}$ ). Assume that $k \geq 3$.Then we have $g_{1} \geq 4 d_{1}-7$ by (3.4). However, as we see above, there is no solution of this and hence we have $1 \leq k \leq 2$.

Q.E.D.

Proposition 23. Let $(G, \rho, V)$ be a reduced triplet satisfying $\operatorname{dim} V$ $\leq \operatorname{dim} G$ with an exceptional simple algebraic group and $k=2$. Then it is a trivial P.V. or it is equivalent to one of the following triplets.

(1) $\left(\left(G_{2}\right) \times G L(2), A_{2} \otimes \Lambda_{1}, V(7) \otimes V(2)\right)$.

(2) $\quad\left(\left(G_{2}\right) \times G L(3), A_{2} \otimes A_{1}, V(7) \otimes V(3)\right)$.

(3) $\quad\left(F_{4} \times G L(2), \Lambda_{4} \otimes \Lambda_{1}, V(26) \otimes V(2)\right)$.

(4) $\quad\left(E_{6} \times G L(2), A_{1} \otimes A_{1}, V(27) \otimes V(2)\right)$.

(5) $\quad\left(E_{6} \times G L(3), \Lambda_{1} \otimes \Lambda_{1}, V(27) \otimes V(3)\right)$.

(6) $\quad\left(E_{6} \times G L(2), \Lambda_{1} \otimes 2 \Lambda_{1}, V(27) \otimes V(3)\right)$.

(7) $\quad\left(E_{7} \times G L(2), \Lambda_{6} \otimes \Lambda_{1}, V(56) \otimes V(2)\right)$.

Proof. First we shall show that $d_{1}<g_{1}$. Assume that $d_{1} \geq g_{1}$. In this case, (3.1) implies that $1+d_{1}+\left(d_{2}^{2}-1\right) \geq d_{1} d_{2}$. Since $d_{1} \geq 14$ and $d_{2} \geq 2$, we have $d_{2} \geq \frac{1}{2}\left(d_{1}+\sqrt{d_{1}^{2}-4 d_{1}}\right) \geq d_{1}-1$ and hence $d_{2}=d_{1}$ or $d_{2}=$ $d_{1}-1$. Hence if $G_{2} \neq S L\left(d_{2}\right)$, together with Proposition 42 in $\S 1$, we 
have $1+d_{1} \geq d_{1} d_{2}-\frac{1}{2} d_{2}\left(d_{2}+1\right)=\frac{1}{2} d_{1}\left(d_{1}-1\right)$, i.e., $d_{1}\left(d_{1}-3\right) \leq 2\left(d_{1} \geq 14\right)$. This is a contradiction and hence $G_{2}=S L\left(d_{2}\right)$. In this case, however, it is a trivial P.V. when $d_{2}=d_{1}$ and it is not reduced if $d_{2}=d_{1}-1$. Hence we may assume that $d_{1}<g_{1}$. Assume that $G_{1}=\left(G_{2}\right)$. Then we have $d_{1}=7$ since $2 \leq d_{1}<g_{1}=14$. If $G_{2} \neq S L\left(d_{2}\right)$, we have $1+14+$ $\frac{1}{2} d_{2}\left(d_{2}+1\right) \geq 7 d_{2}\left(2 \leq d_{2} \leq 7\right)$ and hence $d_{2}=3, G_{2}=S L(2)$. However, in this case we have $18=1+g_{1}+g_{2} \geq d_{1} d_{2}=21$, i.e., a contradiction. Hence $G_{2}=S L\left(d_{2}\right)$. Then we have $2 \leq d_{2} \leq 3$, i.e., (1) and (2) since otherwise it is not reduced. Next assume that $G_{1}=F_{4}$. Then $d_{1}=26$ since $2 \leq d_{1}<g_{1}=56$. If $G_{2} \neq S L\left(d_{2}\right)$, we have $1+52+\frac{1}{2} d_{2}\left(d_{2}+1\right) \geq 26 d_{2}$ $\left(26 \geq d_{2} \geq 3\right)$. There is no solution and hence $G_{2}=S L\left(d_{2}\right)$. In this case we have $2 \leq d_{2} \leq 13$ since otherwise it is not reduced. Since $1+52+$ $\left(d_{2}^{2}-1\right) \geq 26 d_{2}\left(13 \geq d_{2} \geq 2\right)$, we have $d_{2}=2$, i.e., (3). Assume that $G_{1}$ $=E_{6}$. Then $d_{1}=27$. If $G_{2}=S L\left(d_{2}\right)$, we may assume that $13 \geq d_{2} \geq 2$ since otherwise it is not reduced. Then (3.1) implies that $1+78+\left(d_{2}^{2}\right.$ $-1) \geq 27 d_{2}\left(13 \geq d_{2} \geq 2\right)$, and hence $2 \leq d_{2} \leq 3$, i.e., (4) and (5). If $G_{2} \neq$ $S L\left(d_{2}\right)$, we have $1+78+\frac{1}{2} d_{2}\left(d_{2}+1\right) \geq 27 d_{2}\left(27 \geq d_{2} \geq 3\right)$ and hence $d_{2}=$ $3, G_{2}=S L(2)$, i.e., (6). Assume that $G_{1}=E_{7}$. Then $d_{1}=56$. If $G_{2} \neq$ $S L\left(d_{2}\right)$, we have $1+133+\frac{1}{2} d_{2}\left(d_{2}+1\right) \geq 56 d_{2}\left(56 \geq d_{2} \geq 3\right)$. Since there is no solution we have $G_{2}=S L\left(d_{2}\right)$. We may assume that $2 \leq d_{2} \leq 28$. By (3.1) we have $1+133+\left(d_{2}^{2}-1\right) \geq 56 d_{2}\left(28 \geq d_{2} \geq 2\right)$ and hence $d_{2}=2$, i.e., (7). Finally assume that $G_{1}=E_{8}$. Then the least representation of $E_{8}$ is the adjoint representation (see Example 41 in $\S 1$ ), we have $g_{1}=d_{1}$ $<g_{1}$, i.e., a contradiction. Thus we obtain our assertion. Q.E.D.

THEOREM 24. Let $(\tilde{G}, \tilde{\rho}, \tilde{V})$ be a reduced triplet and let $\tilde{\mathrm{g}}$ be the Lie algebra of $\tilde{\rho}(\tilde{G})$. Assume that the center of $\tilde{\mathfrak{g}}$ is one-dimensional. Then it is not a P.V. unless it is equivalent to one of the following reduced triplets.

(1) $\left(G \times G L(m), \rho \otimes \Lambda_{1}, V(n) \otimes V(m)\right)$ where $\rho: G \rightarrow G L(V(n))$ is an $n$-dimensional irreducible representation of a semi-simple algebraic group $G(\neq S L(n))$ with $m \geq n \geq 3$.

(2) $\left(S L(n) \times G L(m), \Lambda_{1} \otimes \Lambda_{1}, V(n) \otimes V(m)\right)$ $(m / 2 \geq n \geq 1$, or $n=m \geq 1)$.

(3) $(G L(1) \times G, \square \otimes$ adjoint rep., $V(1) \otimes V(n))$ where $G$ is an almost simple algebraic group of dimension $n(\geq 3)$.

(4) $\left(G L(n), 2 \Lambda_{1}, V\left(\frac{1}{2} n(n+1)\right)\right)(n \geq 3)$.

(5) $\left(G L(n), \Lambda_{2}, V\left(\frac{1}{2} n(n-1)\right)\right)(n \geq 5)$. 
(6) $\left(G L(2), 3 \Lambda_{1}, V(4)\right)$.

( 7 ) $\left(G L(6), \Lambda_{3}, V(20)\right)$.

( 8 ) $\left(G L(7), \Lambda_{3}, V(35)\right)$.

(9) $\left(G L(8), \Lambda_{3}, V(56)\right)$.

(10) $\quad\left(S L(3) \times G L(2), 2 \Lambda_{1} \otimes \Lambda_{1}, V(6) \otimes V(2)\right)$.

(11) $\quad\left(S L(n) \times G L(2), \Lambda_{2} \otimes \Lambda_{1}, V\left(\frac{1}{2} n(n-1)\right) \otimes V(2)\right)(n \geq 5)$.

(12) $\quad\left(S L(5) \times G L(3), \Lambda_{2} \otimes \Lambda_{1}, V(10) \otimes V(3)\right)$.

(13) $\quad\left(S L(5) \times G L(4), \Lambda_{2} \otimes \Lambda_{1}, V(10) \otimes V(4)\right)$.

(14) $\quad\left(S L(n) \times S L(n) \times G L(2), \Lambda_{1} \otimes \Lambda_{1} \otimes \Lambda_{1}, V(n) \otimes V(n) \otimes V(2)\right)$ $(n \geq 3)$.

(15) $\quad\left(S p(n) \times G L(m), \Lambda_{1} \otimes \Lambda_{1}, V(2 n) \otimes V(m)\right)(n \geq m \geq 1)$.

(16) $\quad\left(G L(1) \times S p(n) \times S O(m), \quad \square \otimes \Lambda_{1} \otimes \Lambda_{1}, V(1) \otimes V(n) \otimes V(m)\right)$ $(2 n \geq m \geq 3)$.

(17) $\quad\left(G L(1) \times S p(n), \square \otimes A_{2}, V(1) \otimes V((n-1)(2 n+1))\right)(n \geq 3)$.

(18) $\quad\left(G L(1) \times S p(n) \times S p(m), \quad \square \otimes \Lambda_{1} \otimes \Lambda_{1}, V(1) \otimes V(2 n) \otimes V(2 m)\right)$ $(n \geq m \geq 2)$.

(19) $\quad\left(G L(1) \times S p(3), \square \otimes \Lambda_{3}, V(1) \otimes V(14)\right)$.

(20) $\quad\left(S O(n) \times G L(m), A_{1} \otimes A_{1}, V(n) \otimes V(m)\right)(n \geq 3, n / 2 \geq m \geq 1)$.

(21) $\quad\left(G L(1) \times S O(n) \times S p(m), \square \otimes \Lambda_{1} \otimes \Lambda_{1}, V(1) \otimes V(n) \otimes V(2 m)\right)$ $(n>2 m \geq 4)$.

(22) $\quad\left(\operatorname{Spin}(7) \times G L(d)\right.$, spin rep. $\left.\otimes A_{1}, V(8) \otimes V(d)\right)(1 \leq d \leq 4)$.

(23) $\quad\left(\operatorname{Spin}(7) \times G L(2)\right.$, spin rep. $\left.\otimes 2 \Lambda_{1}, V(8) \otimes V(3)\right)$.

(24) $\quad\left(G L(1) \times S p i n(7) \times S p(2), \square \otimes \operatorname{spin~rep.~} \otimes \Lambda_{1}, V(1) \otimes V(8) \otimes V(4)\right)$.

(25) $\quad\left(\operatorname{Spin}(10) \times G L(d)\right.$, half-spin rep. $\left.\otimes \Lambda_{1}, V(16) \otimes V(d)\right)(1 \leq d \leq 3)$.

(26) $\quad\left(\operatorname{Spin}(10) \times G L(2)\right.$, half-spin rep. $\left.\otimes 2 \Lambda_{1}, V(16) \otimes V(3)\right)$.

(27) $\quad\left(\operatorname{Spin}(9) \times G L(d)\right.$, spin rep. $\left.\otimes \Lambda_{1}, V(16) \otimes V(d)\right)(1 \leq d \leq 2)$.

(28) $\quad\left(\operatorname{Spin}(12) \times G L(d)\right.$, half-spin rep. $\left.\otimes \Lambda_{1}, V(32) \otimes V(d)\right)(1 \leq d \leq 2)$.

(29) $\quad(G L(1) \times \operatorname{Spin}(11), \square \otimes \operatorname{spin}$ rep., $V(1) \otimes V(32))$.

(30) $\quad(G L(1) \times \operatorname{Spin}(14), \square \otimes$ half-spin rep., $V(1) \otimes V(64))$.

(31) $\quad(G L(1) \times S p i n(13), \square \otimes \operatorname{spin}$ rep., $V(1) \otimes V(64))$.

(32) $\quad\left(\left(G_{2}\right) \times G L(d), \Lambda_{2} \otimes \Lambda_{1}, V(7) \otimes V(d)\right)(1 \leq d \leq 3)$.

(33) $\quad\left(F_{4} \times G L(d), \Lambda_{4} \otimes \Lambda_{1}, V(26) \otimes V(d)\right)(1 \leq d \leq 2)$.

(34) $\quad\left(E_{6} \times G L(d), \Lambda_{1} \otimes \Lambda_{1}, V(27) \otimes V(d)\right)(1 \leq d \leq 3)$.

(35) $\quad\left(E_{6} \times G L(2), \Lambda_{1} \otimes 2 \Lambda_{1}, V(27) \otimes V(3)\right)$.

(36) $\quad\left(E_{7} \times G L(d), \Lambda_{6} \otimes \Lambda_{1}, V(56) \otimes V(d)\right)(1 \leq d \leq 2)$.

Proof. By Propositions 9, 12, 14, 15, $18 \sim 23$, and Example 45 in $\S 1$, we obtain our assertion. Note that the adjoint representation of $S L(n)$ 
$(n \geq 2)$ (resp. $S p(n), S O(n))$ is $\Lambda_{1}+\Lambda_{n-1}$ (resp. $\left.2 \Lambda_{1}, \Lambda_{2}\right)$. A triplet (5) in Proposition 12 is equivalent to the triplet $\left(G L(1) \times S O(6) \times S O(3), \square \otimes \Lambda_{1}\right.$ $\left.\otimes \Lambda_{1}, \quad V(1) \otimes V(6) \otimes V(3)\right)$. Hence it should be omitted in view of Proposition 14 in $\S 2$ and Lemma 17 in $\S 3$.

Q.E.D.

\section{§4. Relative invariants and the regularity}

Before going on to investigate the reduced triplets in Theorem 24 in $\S 3$, we shall prepare some general notions about prehomogeneous vector spaces. Since it is convenient to consider them in a general situation, we do not assume the irreducibility in this section. The results in this section are already published in Japanese (see [11]) except Propositions $15,16,18$, and 23 .

Definition 1. Let $G$ be a connected linear algebraic group. A rational homomorphism $\chi: G \rightarrow C^{\times}\left(C^{\times}=C-\{0\}\right)$ is called a rational character of $G$. The group of all rational characters of $G$ will be denoted by $X(G)$. Rational characters $\chi_{1}, \cdots, \chi_{\ell}$ are called multiplicatively independent if they generate a free abelian group of rank $\ell$ in $X(G)$.

DEFINITION 2. Let $(G, \rho, V)$ be a triplet where $\rho$ is not necessarily irreducible. A non-constant rational function $f(x)$ on $V$ is called a relative invariant of $(G, \rho, V)$ if there exists a rational character $\chi$ of $G$ satisfying $f(\rho(g) x)=\chi(g) f(x)$ for any $g \in G$ and $x \in V$. A relative invariant corresponding to the identity character $\chi=1$, is called an absolute invariant.

Proposition 3. Let $(G, \rho, V)$ be a $P . V$. Then a relative invariant $i s$, up to a constant multiple, uniquely determined by its corresponding character. In particular, any relative invariant is a homogeneous function.

Proof. Let $f_{1}(x)$ and $f_{2}(x)$ be relative invariants corresponding to the same character. Then the quotient $f_{1}(x) / f_{2}(x)$ is an absolute invariant, and hence constant by Proposition 3 in $\S 2$. Let $f(x)$ be any relative invariant. Since $f_{t}(x)=f(t x)\left(t \in C^{\times}\right)$is also a relative invariant with the same character as $f(x)$, we have $f(t x)=c \cdot f(x)$ for some $c \in C$. This implies the homogeneity of $f(x)$.

Q.E.D.

Lemma 4. Relative invariants $f_{1}, \cdots, f_{\ell}$ corresponding to multiplicatively independent characters $\chi_{1}, \cdots, \chi_{\ell}$, are algebraically independent. 
Proof. Assume that $f_{1}, \cdots, f_{\ell}$ are algebraically dependent. Then there exist monomials $U_{1}, \cdots, U_{s}$ of $f_{1}, \cdots, f_{\ell}$ such that they are linearly dependent and any $(s-1)$ of them are linearly independent. By the definition, the subspace $W$ of $C^{s}$ defined by $W=\left\{\left(c_{1}, \cdots, c_{s}\right) \in C^{s} \mid \sum_{i=1}^{s} c_{i} U_{i}=0\right\}$ is one-dimensional. On the other hand, $U_{i}$ is a relative invariant corresponding to some character $\mu_{i}(1 \leq i \leq s)$. Then $\left(c_{1}, \cdots, c_{s}\right) \in W$ implies $\left(c_{1} \mu_{1}(g), \cdots, c_{s} \mu_{s}(g)\right) \in W$ for any $g \in G$. Since $\operatorname{dim} W=1$, we have $\mu_{1}=$ $\cdots=\mu_{s}$. This is a contradiction since multiplicative independence of $\chi_{1}, \cdots, \chi_{\ell}$ implies that $\mu_{1}, \cdots, \mu_{s}$ are different from each other. Q.E.D.

Let $(G, \rho, V)$ be a P. V. and let $S$ be its singular set, i.e., $V-S$ $=\rho(G) \cdot x_{0}\left(x_{0} \in V-S\right)$. Let $S_{1}, \cdots, S_{\ell}$ be the irreducible components of $S$ with codimension one. Then we may assume that each $S_{i}$ is the zeros of some irreducible polynomial $f_{i}(x)(1 \leq i \leq \ell): S_{i}=\left\{x \in V \mid f_{i}(x)=0\right\}$.

Proposition 5. These $f_{1}(x), \cdots, f_{\ell}(x)$ are algebraically independent relative invariants. Moreover, any relative invariant $f(x)$ is of the form $f(x)=c f_{1}(x)^{m_{1}} \cdots f_{\ell}(x)^{m_{\ell}}\left(c \in C,\left(m_{1}, \cdots, m_{\ell}\right) \in \boldsymbol{Z}^{\ell}\right)$.

Proof. First we shall show that each $f_{i}(x)$ is a relative invariant $(1 \leq i \leq \ell)$. Since $G$ is connected and $S_{i}$ is irreducible, the Zariski closure $\overline{\rho(G) \cdot S_{i}}$ of $\rho(G) \cdot S_{i}=\left\{\rho(g) x \mid g \in G, x \in S_{i}\right\}$ is also irreducible and as $S_{i} \subset \overline{\rho(G) \cdot S_{i}} \subset S$, we have $\overline{\rho(G) \cdot S_{i}}=S_{i}$. In particular, we have $\rho(G) \cdot S_{i}=S_{i}$. Therefore, a polynomial $f_{i}\left(\rho(g)^{-1} x\right)$ coincides with $f_{i}(x)$ up to a constant multiple and hence there exists a character $\chi_{i}$ of $G$ satisfying $f_{i}(\rho(g) x)=\chi_{i}(g) f_{i}(x)$ for $g \in G, x \in V$. Since $f_{1}(x), \cdots, f_{\ell}(x)$ are irreducible and different from each other, corresponding characters $\chi_{1}, \cdots, \chi_{e}$ are multiplicatively independent. Hence $f_{1}(x), \cdots, f_{\ell}(x)$ are algebraically independent by Lemma 4 . Finally, let $f(x)$ be any relative invariant. Since $G$ is connected, every prime divisor of $f(x)$ is also a relative invariant. Hence we may assume that $f(x)$ is an irreducible polynomial. Then the zeros of $f(x)$ must coincide with $S_{i}$ for some $i(1 \leq i \leq \ell)$ since it is a $G$-invariant irreducible hypersurface. This implies that $f(x)=c f_{i}(x)$ for some $c \in C$.

Q.E.D.

COROLlary 6. Let $(G, \rho, V)$ be a $P . V$. and let $S$ be its singular set. Then there exists a relative invariant of $(G, \rho, V)$ if and only if $S$ has an irreducible component of codimension one.

DEFINITION 7. A prehomogeneous vector space is called regular if 
there exists a relative invariant $f(x)$ such that the Hessian $H_{f}(x)=$ $\operatorname{det}\left(\frac{\partial^{2} f}{\partial x_{i} \partial x_{j}}(x)\right)$ of $f(x)$ is not identically zero.

Proposition 8. Let $(G, \rho, V)$ be a regular $P . V$. Then there exists a relative invariant corresponding to the character $\chi_{0}(g)=\operatorname{det} \rho(g)^{2}(g \in G)$ where $\operatorname{det} \rho(g)$ denotes the determinant of $\rho(g)$ in $V$.

Proof. Let $f(x)$ be a relative invariant satisfying $H_{f}(x) \neq 0$, and let $\chi$ be the character of $f(x)$. By choosing a basis of $V$, we may assume that $V \simeq C^{n}$ and $G \subset G L(n, C)$ where $n=\operatorname{dim} V$. Then we have

$$
\begin{aligned}
\frac{\partial^{2}}{\partial x_{i} \partial x_{j}}(f(g x)) & =\frac{\partial}{\partial x_{i}} \sum_{k} \frac{\partial f}{\partial x_{k}}(g x) \cdot \frac{\partial \sum_{\ell} g_{k \ell} x_{\ell}}{\partial x_{j}} \\
& =\sum_{k} g_{k j} \frac{\partial}{\partial x_{i}} \cdot \frac{\partial f}{\partial x_{k}}(g x) \\
& =\sum_{k, \ell} g_{\ell i} \cdot \frac{\partial^{2} f}{\partial x_{\ell} \partial x_{k}}(g x) \cdot g_{k j}
\end{aligned}
$$

i.e.,

$$
\left(\frac{\partial^{2}}{\partial x_{i} \partial x_{j}}(f(g x))\right)={ }^{t} g\left(\frac{\partial^{2} f}{\partial x_{k} \partial x_{\ell}}(g x)\right) g
$$

for $g=\left(g_{i j}\right) \in G$. Since $f(g x)=\chi(g) f(x)$, we have

$$
\left(\frac{\partial^{2} f}{\partial x_{i} \partial x_{j}}(g x)\right)=\chi(g) \cdot{ }^{t} g^{-1}\left(\frac{\partial^{2} f}{\partial x_{i} \partial x_{j}}(x)\right) g^{-1}
$$

and hence by taking the determinant, we have $H_{f}(g x)=\chi(g)^{n} \cdot \operatorname{det}(g)^{-2}$. $H_{f}(x)$. This implies that $H_{f}(x)$ is a relative invariant corresponding to the character $\chi(g)^{n} \cdot \operatorname{det} \rho(g)^{-2}$. Hence the quotient $f^{n}(x) / H_{f}(x)$ is a relative invariant with the character $\chi_{0}(g)=\operatorname{det} \rho(g)^{2}$.

Q.E.D.

Let $(G, \rho, V)$ be a P. V. and let $S$ be its singular set. Assume that this $\mathrm{P}$. V. has a relative invariant $f(x)$. By choosing a basis, we may identify $V$ with $C^{n}$. Moreover, by the inner product $\langle x, y\rangle=\sum_{i=1}^{n} x_{i} y_{i}$ $\left(x, y \in C^{n}\right)$, we identify the dual vector space $V^{*}$ of $V$ with $C^{n}$. Then we can define a rational map $\varphi: V-S \rightarrow V^{*}$ by

$$
\varphi(x)=\left(\frac{1}{f(x)} \cdot \frac{\partial f}{\partial x_{1}}(x), \cdots, \frac{1}{f(x)} \frac{\partial f}{\partial x_{n}}(x)\right) .
$$

Sometimes we denote $\varphi$ by $\operatorname{grad} \log f$. 
Let $\chi$ be the character of $f(x)$ and let $\delta \chi$ be its infinitesimal character, i.e., $\chi(\exp t A)=\exp t \delta \chi(A)$ for any $t \in C, A \in \mathfrak{g}$, where $\mathfrak{g}$ is the Lie algebra of $G$. Similarly, let $d \rho$ be the infinitesimal representation of $\rho$ (see Definition 44 in $\S 1$ ).

Proposition 9. A rational map $\varphi=\operatorname{grad} \log f$ of $V-S$ into $V^{*}$ does not depend on the choice of coordinate systems and it satisfies the following conditions.

(1) $\varphi(\rho(g) x)=\rho^{*}(g) \varphi(x)$ for $x \in V-S, g \in G$, where $\rho^{*}$ is the contragredient representation of $\rho$.

(2) $\langle d \rho(A) x, \varphi(x)\rangle=\delta \chi(A)$ for $A \in \mathfrak{g}, x \in V-S$.

Proof. We may assume that $G \subset G L(n, C)$ and $g \subset g(r, C)$. Since $\frac{\partial}{\partial x_{i}} f(g x)=\sum_{k} \frac{\partial f}{\partial x_{k}}(g x) \frac{\partial(g x)_{k}}{\partial x_{i}}=\sum_{k} \frac{\partial f}{\partial x_{k}}(g x) g_{k i}$ for $g=\left(g_{i j}\right) \in G$, we have $\operatorname{grad} f(g x)=\chi(g)^{t} g^{-1} \operatorname{grad} f(x)$ where $\operatorname{grad} f(x)=\left(\frac{\partial f}{\partial x_{1}}(x), \cdots, \frac{\partial f}{\partial x_{n}}(x)\right)$ and hence we obtain (1). By differentiation of the equality $f(g x)=$ $\chi(g) f(x)$ for $g=\exp t A \in G$, we obtain (2), i.e., $\langle A x$, $\operatorname{grad} f(x)\rangle=$ $\delta \chi(A) f(x)$. Note that this condition (2) characterizes $\varphi=\operatorname{grad} \log f$ since $\{d \rho(A) x \mid A \in \mathfrak{g}\}=V$ for any $x \in V-S$. This shows that the definition of $\operatorname{grad} \log f$ does not depend on the choice of coordinate systems.

Q.E.D.

Proposition 10. The following conditions are equivalent.

(1) $\operatorname{grad} \log f: V-S \rightarrow V^{*}$ is generically surjective, i.e., the image is Zariski dense.

(2) $H_{\log f(x)} \neq 0(x \in V-S)$.

(3) $H_{f}(x) \neq 0(x \in V-S, \operatorname{deg} f \geq 2) ; \operatorname{det}\left(\frac{\partial f}{\partial x_{i}} \cdot \frac{\partial f}{\partial x_{j}}\right) \neq 0(\operatorname{deg} f=1)$.

Proof. For $x \in V-S$, let $(d \varphi)_{x}: T_{x}(V-S) \rightarrow T_{\varphi(x)} V^{*}$ be the differential map of $\varphi=\operatorname{grad} \log f$. By (1) in Proposition 9, $\varphi$ is generically surjective if $\operatorname{det}(d \varphi)_{x} \neq 0$ for some $x \in V-S$. Since

$$
\begin{aligned}
\operatorname{det}(d \varphi)_{x} & =\operatorname{det}\left(\frac{\partial \varphi_{i}}{\partial x_{j}}(x)\right)=\operatorname{det}\left(\frac{\partial}{\partial x_{j}}\left(\frac{1}{f} \frac{\partial f}{\partial x_{i}}\right)(x)\right) \\
& =\operatorname{det}\left(\frac{\partial^{2} \log f}{\partial x_{j} \partial x_{i}}\right)=H_{\log f},
\end{aligned}
$$

the equivalence of (1) and (2) is obvious. Moreover, since 


$$
\begin{aligned}
\operatorname{det}(d \varphi)_{x} & =\operatorname{det}\left(\frac{\partial}{\partial x_{j}}\left(\frac{1}{f} \frac{\partial f}{\partial x_{i}}\right)(x)\right) \\
& =\operatorname{det}\left(\frac{1}{f} \cdot \frac{\partial^{2} f}{\partial x_{i} \partial x_{j}}(x)-\frac{1}{f^{2}} \cdot \frac{\partial f}{\partial x_{i}} \frac{\partial f}{\partial x_{j}}\right),
\end{aligned}
$$

if $\operatorname{deg} f=1$, we have

$$
\operatorname{det}(d \varphi)_{x}=(-1)^{n} f(x)^{-2 n} \cdot \operatorname{det}\left(\frac{\partial f}{\partial x_{i}} \frac{\partial f}{\partial x_{j}}\right),
$$

i.e., (3) for $\operatorname{deg} f=1$. Assume that $r=\operatorname{deg} f(x) \geq 2$. Then the Euler's identity says that

$$
\begin{gathered}
\sum_{i=1}^{n} x_{i}^{t}\left(\frac{1}{f} \cdot \frac{\partial^{2} f}{\partial x_{1} \partial x_{i}}, \cdots, \frac{1}{f} \cdot \frac{\partial^{2} f}{\partial x_{n} \partial x_{i}}, \frac{1}{f} \cdot \frac{\partial f}{\partial x_{i}}\right) \\
\quad=\left(\frac{r-1}{f} \cdot \frac{\partial f}{\partial x_{1}}, \cdots, \frac{r-1}{f} \cdot \frac{\partial f}{\partial x_{n}}, r\right)
\end{gathered}
$$

and hence we have

$$
\begin{aligned}
& \operatorname{det}(d \varphi)_{x}=\operatorname{det}\left(\frac{1}{f} \cdot \frac{\partial^{2} f}{\partial x_{i} \partial x_{j}}(x)-\frac{1}{f^{2}} \frac{\partial f}{\partial x_{i}} \frac{\partial f}{\partial x_{j}}\right) \\
& =\operatorname{det}\left(\frac{\left(\frac{1}{f} \cdot \frac{\partial^{2} f}{\partial x_{i} \partial x_{j}}-\frac{1}{f^{2}} \cdot \frac{\partial f}{\partial x_{i}} \cdot \frac{\partial f}{\partial x_{j}}\right.}{0} \mid \begin{array}{c}
\frac{1}{f} \frac{\partial f}{\partial x_{1}} \\
\vdots \\
\frac{1}{f} \cdot \frac{\partial f}{\partial x_{n}} \\
1
\end{array}\right) \\
& =\operatorname{det}\left(\begin{array}{c|c}
\left(\frac{1}{f} \cdot \frac{\partial^{2} f}{\partial x_{i} \partial x_{j}}\right) & \frac{\partial f}{f} \frac{\partial x_{1}}{\vdots} \\
\frac{1}{\frac{1}{f} \cdot \frac{\partial f}{\partial x_{1}}, \cdots, \frac{1}{f} \frac{\partial f}{\partial x_{n}}} & 1
\end{array}\right) \\
& =\operatorname{det}\left(\frac{\partial^{2} f}{\partial x_{i} \partial x_{j}}(x)\right) \cdot \frac{1}{f(x)^{n}} \cdot\left(1-\frac{r}{r-1}\right) \text {, }
\end{aligned}
$$

i.e.,

$$
\operatorname{det}(d \varphi)_{x}=\frac{-1}{r-1} \cdot \frac{H_{f}(x)}{f(x)^{n}}(x \in V-S)
$$


Hence we obtain our assertion.

Q.E.D.

Remark 11. If $\operatorname{deg} f=1$ and $\operatorname{det}\left(\frac{\partial f}{\partial x_{i}} \cdot \frac{\partial f}{\partial x_{j}}\right) \neq 0$, then $H_{f^{2}}(x) \neq 0$ since $\frac{\partial^{2} f^{2}}{\partial x_{i} \partial x_{j}}=\frac{\partial}{\partial x_{i}}\left(2 f \cdot \frac{\partial f}{\partial x_{j}}\right)=2 \frac{\partial f}{\partial x_{i}} \cdot \frac{\partial f}{\partial x_{j}}\left(\right.$ note that $\frac{\partial^{2} f}{\partial x_{i} \partial x_{j}}=0$ since $\operatorname{deg} f=1)$. Hence $(G, \rho, V)$ is regular if and only if there exists a relative invariant $f(x)$ such that $\operatorname{grad} \log f$ is generically surjective. In general, a prehomogeneous vector space $(G, \rho, V)$ is called quasi-regular if there exist $\omega \in \mathrm{g}^{*}$ (= the dual space of $\mathrm{g}$ ) and a regular rational map $\varphi: V-S \rightarrow V^{*}$ satisfying the following conditions:

(1) $\varphi(\rho(g) x)=\rho^{*}(g) \varphi(x)(g \in G, x \in V-S)$,

(2) $\langle d \rho(A), \varphi(x)\rangle=\omega(A)(A \in \mathfrak{g}, x \in V-S)$,

(3) $\varphi$ is generically surjective, i.e., $\overline{\varphi(V-S)}=V^{*}$.

In this case it can be proved that a triplet $\left(G, \rho^{*}, V^{*}\right)$ is also a $\mathrm{P}$. V. and $\varphi$ is a biregular rational map of $V-S$ onto $V^{*}-S^{*}$. Moreover, the number of irreducible hypersurfaces in $S$ is the same as in $S^{*}$. Note that in general the dual triplet $\left(G, \rho^{*}, V^{*}\right)$ is not a P. V. even if $(G, \rho, V)$ is a P. V. For example, $G=\left\{\left(\begin{array}{ll}1 & b \\ & a\end{array}\right) \mid a, b \in C, a \neq 0\right\}, V=\dot{C}^{2}$, $\rho\left(\left(\begin{array}{ll}1 & b \\ & a\end{array}\right)\right)\left(\begin{array}{l}x \\ y\end{array}\right)=\left(\begin{array}{c}x+b y \\ a y\end{array}\right)$. By Propositions 9 and 10, a regular P. V. is always quasi-regular. But the converse is not true. For example, put $\tilde{G}=\left\{\left(\begin{array}{ll}a & b \\ & a\end{array}\right) \mid a, b \in C, a \neq 0\right\}, \tilde{V}=C^{2}, \tilde{\rho}\left(\left(\begin{array}{ll}a & b \\ a\end{array}\right)\right)\left(\begin{array}{l}x \\ y\end{array}\right)=\left(\begin{array}{c}a x+b y \\ a y\end{array}\right)$. Then this triplet $(\tilde{G}, \tilde{\rho}, \tilde{V})$ is a quasi-regular P. V. but not regular. However, if $G$ is reductive, then the regularity and the quasi-regularity are equivalent. Hence we omit the detail of quasi-regularity.

Now we shall return to the irreducible case.

Proposition 12. Let $(G, \rho, V)$ be an irreducible $P$. V. Then there is, up to a constant multiple, at most one irreducible relative invariant polynomial $f(x)$, and hence any relative invariant is of the form $c \cdot f(x)^{m}$ $\left(c \in \boldsymbol{C}^{\times}, m \in \boldsymbol{Z}\right)$.

Proof. Assume that there exist two such polynomials $f_{1}(x), f_{2}(x)$. Let $r_{i}$ be the degree of $f_{i}(x)(1 \leq i \leq 2)$. Then the quotient $f(x)=$ $f_{1}(x)^{r_{2}} / f_{2}(x)^{r_{1}}$ is not constant by Propositions 5. Moreover, it is an absolute invariant by Theorem 1 in $\S 1$. This is a contradiction in view 
of Proposition 3 in $\S 2$.

Q.E.D.

Definition 13. Let $(G, \rho, V)$ be an irreducible P. V. By the relative invariant of $(G, \rho, V)$, we mean the irreducible relative invariant polynomial which is, if it exists, uniquely determined up to a constant multiple by Proposition 12. Note that its degree is uniquely determined by $(G, \rho, V)$.

Proposition 14. Let $(G, \rho, V)$ be an irreducible regular $P$. V. and let $f(x)$ be the relative invariant and $\chi$ its character. Put $r=\operatorname{deg} f(x)$ and $n=\operatorname{dim} V$. Then we have $r \mid 2 n$ and $\chi(g)^{2 n / r}=\operatorname{det} \rho(g)^{2}$.

Proof. By Proposition 8, there exists a relative invariant $F(x)$ with the character $\operatorname{det} \rho(g)^{2}$. Then by Proposition 12, we have $F(x)=c \cdot f(x)^{m}$ for some $c \in C^{\times}, m \in Z$. Hence we have $\chi(g)^{m}=\operatorname{det} \rho(g)^{2}$. If we take $g$ such as $\rho(g)=t I_{n}\left(t \in C^{\times}\right)$, we have $t^{m r}=t^{2 n} \quad\left(t \in C^{\times}\right)$and hence $m=$ $\frac{2 n}{r}$.

Q.E.D.

Proposition 15 (The degree formula). Let $(G, \rho, V)$ be an irreducible regular $P$. $V$. and let $f(x)$ be the relative invariant. Assume that there exists an orbit $\rho(G) x_{0}$ of codimension one, i.e., $\overline{\rho(G) x_{0}}=\{x \in V \mid f(x)=0\}$. Then

$$
\operatorname{deg} f(x)=\frac{\operatorname{tr} d \rho(A)+\operatorname{tr} \operatorname{ad}_{8_{x_{0}}} A}{\operatorname{tr} d \rho(A)} \cdot \operatorname{dim} V \quad\left(A \in \mathfrak{g}_{x_{0}}, \operatorname{tr} d \rho(A) \neq 0\right)
$$

where $\operatorname{tr} d \rho(A)$ is the trace of $d \rho(A)$ in $V$ and $\operatorname{tr} \operatorname{ad}_{g_{x_{0}}} A$ is the a trace of the adjoint representation of the isotropy subalgebra $g_{x_{0}}$ at $x_{0}$.

Proof. By differentiating the equality $\chi(g)^{2 n / r}=\operatorname{det} \rho(g)^{2}$ in Proposition 14 , we have $\frac{n}{r} \delta \chi(A)=\operatorname{tr} d \rho(A)(A \in \mathfrak{g}=$ the Lie algebra of $G)$, i.e., $\operatorname{deg} f=\frac{\delta \chi(A)}{\operatorname{tr} d \rho(A)} \cdot \operatorname{dim} V(A \in \mathfrak{g}) . \quad$ Let $V_{x_{0}}=V / d \rho(\mathfrak{g}) \cdot x_{0}$ be the normal vector space at $x_{0}$. Then the isotropy subgroup $G_{x_{0}}$ at $x_{0}$ acts on $V_{x_{0}}$ since $\rho\left(G_{x_{0}}\right) \cdot d \rho(\mathfrak{g}) \cdot x_{0} \subset d \rho(\mathfrak{g}) \cdot x_{0}$. We shall show that $\chi(g)=\operatorname{det}_{V_{x_{0}}} g$ for $g \in G_{x_{0}}$. Since $f\left(x_{0}\right)=0$ and $d f\left(x_{0}\right) \neq 0$, we have $f(x)=\left\langle x-x_{0}, d f\left(x_{0}\right)\right\rangle$ + higher term of $\left(x-x_{0}\right)$. Since $f(\rho(g) x)=\chi(g) f(x)$ and $\rho(g) x-x_{0}=$ $\rho(g)\left(x-x_{0}\right)$ for $g \in G_{x_{0}}$, we have $\left\langle\rho(g) x-x_{0}, d f\left(x_{0}\right)\right\rangle=\chi(g)\left\langle x-x_{0}, d f\left(x_{0}\right)\right\rangle$. On the other hand, Euler's identity says that $\left\langle x_{0}, d f\left(x_{0}\right)\right\rangle=\operatorname{deg} f \cdot f\left(x_{0}\right)$ 
$=0$, and hence we have $\left\langle\rho(g) x, d f\left(x_{0}\right)\right\rangle=\chi(g)\left\langle x, d f\left(x_{0}\right)\right\rangle$, i.e., $\langle\rho(g) x-$ $\left.\chi(g) x, d f\left(x_{0}\right)\right\rangle=0 \quad\left(g \in G_{x_{0}}\right)$. Since $\left\langle d \rho(A) x_{0}, d f\left(x_{0}\right)\right\rangle=\delta \chi(A) f\left(x_{0}\right)=0$ for any $A \in \mathfrak{g}$ (see Proposition 9, (2); note that $\langle d \rho(A) x \operatorname{grad} f(x)\rangle=$ $\langle d \rho(A) x, d f(x)\rangle$ by definition), and $\operatorname{codim}_{V} d \rho(\mathrm{g}) x_{0}=1$, we have $\rho(g) x \equiv$ $\chi(g) x \bmod d \rho(\mathrm{g}) \cdot x_{0}$. In fact if $\rho(g) x-\chi(g) x \notin d \rho(\mathrm{g}) x_{0}$ we have $\left\langle V d f\left(x_{0}\right)\right\rangle$ $=0$, i.e., $d f\left(x_{0}\right)=0$. This is a contradiction. Hence we have $\operatorname{det}_{V_{x_{0}}} g$ $=\chi(g)$ (note that $\operatorname{dim} V_{x_{0}}=1$ ). By differentiation of this equality, we have $\delta \chi(A)=\operatorname{tr}_{V_{x_{0}}} A\left(A \in \mathrm{g}_{x_{0}}\right)$. Since $\mathrm{g}$ is reductive, we have $\operatorname{tr} \operatorname{ad}_{\mathrm{g}} A=0$ and hence $\operatorname{tr}_{V_{x_{0}}} A=\operatorname{tr} d \rho(A)-\operatorname{tr}_{d \rho(g) \cdot x_{0}} A=\operatorname{tr} d \rho(A)+\operatorname{tr} \operatorname{ad}_{g_{x x_{0}}} A$, i.e., $\delta \chi(A)$ $=\operatorname{tr} d \rho(A)+\operatorname{tr} \operatorname{ad}_{\delta_{x_{0}}} A\left(A \in \mathfrak{g}_{x_{0}}\right)$. Together with $\operatorname{deg} f=\frac{\delta \chi(A)}{\operatorname{tr} d \rho(A)} \cdot \operatorname{dim} V$, we obtain our assertion.

Q.E.D.

Proposition 16. Let $(G, \rho, V)$ be an irreducible $P$. V. satisfying $\operatorname{dim} G=\operatorname{dim} V(=n)$. Then it is regular and there is a relative invariant polynomial $f(x)$ of degree $n$. Moreover, if there exists an orbit of codimension one, then $f(x)$ is irreducible.

Proof. Since $G$ is reductive and the generic isotropy subgroup is finite, it is regular by the following Proposition 25. By choosing a basis we may assume that $V \simeq C^{n}$ and $G \subset G L(n, C)$. Let $g(\subset g r(n, C))$ be the Lie algebra of $G$, and let $A_{1}, \cdots, A_{n}$ be a basis of $\mathrm{g}$ over $C$. Then $A_{1}, \cdots, A_{n}$ are $n \times n$ matrices. Define a polynomial $f(x)$ by $f(x)=$ $\operatorname{det}\left(A_{1} x, \cdots, A_{n} x\right)$ for $x \in \boldsymbol{C}^{n}$. We shall show that $f(x)$ is a relative invariant. Let $\left(c_{i j}(g)\right)$ be an $n \times n$ matrix of the adjoint representation of $G$ w. r. t. a basis $A_{1}, \cdots, A_{n}$, i.e., $\left(g^{-1} A_{1} g, \cdots, g^{-1} A_{n} g\right)=\left(A_{1}, \cdots, A_{n}\right)$ $\left(c_{i j}(g)\right)$. Then we have

$$
\begin{aligned}
f(g x) & =\operatorname{det} g \cdot \operatorname{det}\left(g^{-1} A_{1} g x, \cdots, g^{-1} A_{n} g x\right) \\
& =\operatorname{det} g \cdot\left(\sum_{i} c_{i i}(g) A_{i} x, \cdots, \sum c_{i n}(g) A_{i} x\right) \\
& =\operatorname{det} g \cdot \operatorname{det}\left(c_{i j}(g)\right) \cdot f(x) .
\end{aligned}
$$

Assume that there exists an orbit $G x_{0}$ of codimension one. Then $\operatorname{dim} \mathfrak{g}_{x_{0}}=\operatorname{dim} \mathrm{g}-(n-1)=1$ and hence $\mathfrak{g}_{x_{0}}$ is abelian. This implies that $\operatorname{tr} \operatorname{ad}_{g_{x_{0}}} A=0\left(A \in \mathfrak{g}_{x_{0}}\right)$, and hence the degree of the relative invariant is $n$ by Proposition 15 . Therefore $f(x)$ is the relative invariant of $(G, \rho, V)$, i.e., irreducible.

Q.E.D.

Remark 17. Consider a triplet $(G, \rho, V)$ where $G=G L(2, C), V=$ 
$M(2, C), \rho(A) X=A X(A \in G, X \in V)$. Although it is not irreducible, it is regular and its singular set $S$ is an irreducible hypersurface: $S=$ $\{x \in V \mid \operatorname{det} x=0\}$. Moreover, we have

$$
\operatorname{dim} G=\operatorname{dim} V, \operatorname{deg} f=\frac{\delta \chi(A)}{\operatorname{tr}_{V} A} \operatorname{dim} V
$$

where $\delta \chi$ is the differential character of $f(x)=\operatorname{det} x$. However, there is no orbit of codimension 1 , and $\operatorname{deg} f=\frac{1}{2} \operatorname{dim} V$.

Now we shall consider relative invariants and regularity of the castling transform.

Let $\left(G \times S L(n), \rho \otimes \Lambda_{1}, V(m) \otimes V(n)\right)$ be a triplet with $m>n \geq 1$. Identify $V(m) \otimes V(n)$ with all $m \times n$ matrices $M(m, n, C)$. We may assume that $G \subset G L(m)$. Then we have $\rho \otimes \Lambda_{1}\left(g_{1}, g_{2}\right) X=g_{1} X^{t} g_{2}$ for $X \in M(m, n, C), g_{1} \in G, g_{2} \in S L(n)$. Let $f\left(x_{11}, x_{12}, \cdots, x_{m n}\right)\left(X=\left(x_{i j}\right) \in M(m\right.$, $n, C)$ ) be a relative invariant of this triplet. Put

$$
X_{i_{1}, \cdots, i_{n}}=\operatorname{det}\left(\begin{array}{l}
x_{i_{1} 1}, \cdots, x_{i_{1} n} \\
\vdots \\
x_{i_{n} 1}, \cdots, x_{i_{n} n}
\end{array}\right) \quad\left(1 \leq i_{1}, \cdots, i_{n} \leq m\right) .
$$

Since $f$ is invariant under the action of $S L(n)$, the first main theorem for $S L(n)$ (see p. 45 in [1]) says that there exists a polynomial $F$ of $\left(\begin{array}{c}m \\ n\end{array}\right)$-variables satisfying $f\left(\left(x_{i j}\right)\right)=F\left(\left(X_{i_{1}, \cdots, i_{n}}\right)\right)\left(1 \leq i, i_{1}, \cdots, i_{n} \leq m, 1 \leq j\right.$ $\leq n)$. Now we shall consider the castling transform $(G \times S L(m-n)$, $\left.\rho^{*} \otimes \Lambda_{1}, V(m) \otimes V(m-n)\right)$ of this triplet. Similarly, we may assume that $V(m) \otimes V(m-n)=M(m, m-n, C)$ and $G \subset G L(m)$. Then we have

$$
\left(\rho^{*} \otimes \Lambda_{1}\right)\left(g_{1}, g_{2}\right) X={ }^{t} g_{1}^{-1} X^{t} g_{2} \quad \text { for } X=\left(x_{\substack{i j \\ j \leq i \leq m \\ n+1 \leq j \leq m}} \in M(m, m-n, C) .\right.
$$

Put

$$
X_{i_{n+1}, \cdots, i_{m}}=\operatorname{det}\left(\begin{array}{l}
x_{i_{n+1}, n+1}, \cdots, x_{i_{n+1}, m} \\
\vdots \\
x_{i_{m}, n+1}, \cdots, x_{i_{m}, m}
\end{array}\right)
$$

and to each $X_{i_{1}, \cdots, i_{n}}$, correspond $X_{i_{n+1}, \cdots, i_{m}}$ under the condition that $\left\{i_{1}, \cdots, i_{n}, i_{n+1}, \cdots, i_{m}\right\}=\{1, \cdots, m\}$ and $\operatorname{sgn}\left(\begin{array}{l}1, \cdots, m \\ i_{1}, \cdots, i_{m}\end{array}\right)=1$. This gives a one-to-one correspondence between $X_{i_{1}, \cdots, i_{n}}$ and $X_{i_{n+1}, \cdots, i_{m}}$. 
Hence in this case, we denote $X_{i_{n+1}, \ldots, i_{m}}$ by $X_{i_{1}, \ldots, i_{n}}^{\prime}$. We shall show that $\tilde{f}\left(x_{i j}\right)=F\left(\left(X_{i_{1}, \ldots, i_{n}}^{\prime}\right)\right)(1 \leq i \leq m, n+1 \leq j \leq m)$ is a relative invariant of $\left(G \times S L(m-n), \rho^{*} \otimes \Lambda_{1}, V(m) \otimes V(m-n)\right)$. Since each $X_{i_{1}, \cdots, i_{n}}^{\prime}$ is invariant, $\tilde{f}$ is also invariant under the action of $S L(m-n)$. To see $\tilde{f}$ invariant under the action $\rho^{*}$ of $G$, we may assume that $G \subset S L(m)$. Then an element of the Lie algebra $g$ of $G$ is an $m \times m$ matrix of trace 0 . Since $\tilde{f}$ is absolutely invariant under the action $\rho^{*}$ of $G$ if and only if it becomes zero under the action $d \rho^{*}$ of $\mathrm{g}$, we may consider infinitesimally.

It is sufficient to show that the action $d \rho$ of $\mathfrak{g}$ to $X_{i_{1}, \cdots, i_{n}}$ is the same as the contragredient action $d \rho^{*}$ of $\mathfrak{g}$ to $X_{i_{1}, \cdots, i_{n}}^{\prime}$ since in that case $f=$ $F\left(\left(X_{i_{1}, \ldots, i_{n}}\right)\right)$ is invariant under the action $\rho$ of $G$ if and only if $\tilde{f}=$ $F\left(\left(X_{i_{1}, \ldots, i_{n}}^{\prime}\right)\right)$ is invariant under the contragredient action $\rho^{*}$ of $G$. To show this, we may assume that $X_{i_{1}, \cdots, i_{n}}=X_{1, \cdots, n}$. Then $d_{\rho} \rho$ induces

$$
\begin{gathered}
X_{1, \ldots, n} \mapsto \sum_{k=1}^{n} \sum_{\ell=1}^{m} a_{k \ell} X_{1, \ldots, \ell, \cdots, n} \stackrel{v}{v}=\left(a_{11}+\cdots+a_{n n}\right) X_{1, \cdots, n} \\
+\sum_{k=1}^{n} \sum_{\ell=n+1}^{m} a_{k \ell} X_{1, \ldots, \ell, \cdots, n}^{k} \quad \text { for } A=\left(a_{i j}\right) \in \mathfrak{g} .
\end{gathered}
$$

Note that $\sum_{l=1}^{m} a_{i i}=0$. On the other hand, the contragredient action $d \rho^{*}$ induces

$$
\begin{aligned}
X_{1, \cdots, n}^{\prime} & =X_{n+1, \ldots, m} \mapsto \sum_{r=n+1}^{m} \sum_{s=1}^{m}\left(-a_{s r}\right) X_{n+1, \ldots, s, \cdots, m} \underset{r}{r} \\
& =-\left(a_{n+1, n+1}+\cdots+a_{m m}\right) X_{n+1, \ldots, m}+\sum_{r=n+1}^{m} \sum_{s=1}^{n}\left(-a_{s r}\right) X_{n+1, \ldots, s, \cdots, m}^{r} \\
& =\left(a_{11}+\cdots+a_{n n}\right) X_{1, \cdots, n}^{\prime}+\sum_{s=1}^{n} \sum_{r=n+1}^{m} a_{s r}\left(X_{1, \ldots, r, \ldots, n}\right)
\end{aligned}
$$

for $A=\left(a_{i j}\right) \in \mathfrak{g}$.

Hence we obtain our assertion that $\tilde{f}$ is a relative invariant of $\left(G \times S L(m-n), \rho^{*} \otimes \Lambda_{1}, V(m) \otimes V(m-n)\right)$. Since this correspondence is one-to-one, if $f$ is irreducible, then $\tilde{f}$ is also irreducible. Therefore, we obtain the following proposition.

Proposition 18. There is a one-to-one correspondence between relative invariants $f(x)$ of $\left(G \times S L(n), \rho \otimes \Lambda_{1}, V(m) \otimes V(n)\right)(m>n \geq 1)$ and relative invariants $\tilde{f}(\tilde{x})$ of its castling transform $\left(G \times S L(m-n), \rho^{*} \otimes \Lambda_{1}\right.$, $V(m) \otimes V(m-n))$. Moreover, there exists a positive integer $d$ for each $f(x)$ such that $\operatorname{deg} f(x)=n d$ and $\operatorname{deg} \tilde{f}(\tilde{x})=(m-n) d$. If $f$ is irreducible, 
then $\tilde{f}$ is also irreducible.

Next we shall consider some conditions that a given $P . V$. has no relative invariant.

Proposition 19. Let $(G, \rho, V)$ be a P.V. where $\rho$ is not necessariyl irreducible. Let $\chi(\neq 1)$ be a rational character of $G$. Then there exists a relative invariant $f(x)$ corresponding to $\chi$ if and only if the restriction $\left.\chi\right|_{a_{x_{0}}}$ of $\chi$ to a generic isotropy subgroup $G_{x_{0}}\left(x_{0} \in V-S\right)$ is identity. Note that the property $\chi \mid G_{x_{0}}=1$ does not depend on a generic point $x_{0}$ since $G_{\rho(g) x_{0}}=g G_{x_{0}} g^{-1}$.

Proof. If there exists a relative invariant $f(x)$ satisfying $f(\rho(g) x)$ $=\chi(g) f(x)$, we have $f\left(x_{0}\right)=\chi(g) f\left(x_{0}\right)$ and $f\left(x_{0}\right) \neq 0$, $\infty$ for $g \in G_{x_{0}}, x_{0} \in$ $V-S$. This implies that $\chi(g)=1$ for any $g \in G_{x_{0}}$. Conversely, assume that $\chi \mid G_{x_{0}}=1$ for $x_{0} \in V-S$. Then $\chi$ can be regarded as a rational regular function on $G / G_{x_{0}} \approx V-S$, and hence there exists a rational function $f(x)$ on $V$ satisfying $f\left(\rho(g) x_{0}\right)=\chi(g)$ for any $g \in G$. Clearly, $f(x)$ is a relative invariant corresponding to the character $\chi$. Q.E.D.

Proposition 20. Let $(G, \rho, V)$ be an irreducible P.V. and let $g$ be the Lie algebra of $G$. Denote by $\mathfrak{g l}(V)$ the Lie algebra of all endomorphisms of $V$ of trace 0 . Then

(1) If $d \rho(\mathfrak{g}) \subset \mathfrak{g l}(V)$, there exists no (non-constant) relative invariant and hence $(G, \rho, V)$ is not regular.

(2) Let $\mathfrak{g}_{x_{0}}\left(x_{0} \in V-S\right)$ be a generic isotropy subalgebra. If $d \rho\left(\mathfrak{g}_{x_{0}}\right)$ $\not \subset \mathfrak{I l}(V)$, there exists no (non-constant) relative invariant and hence $(G, \rho, V)$ is not regular.

(3) If $d \rho(\mathfrak{g}) \not \subset \mathfrak{s l}(V)$ and $d \rho\left(\mathfrak{g}_{x_{0}}\right) \subset \mathfrak{g l}(V)$, then there exists a (nonconstant) relative invariant of $(G, \rho, V)$.

Proof. (1) By Theorem 1 in $\S 1, d \rho(\mathfrak{g}) \subset \mathfrak{g l}(V)$ implies that $d \rho(\mathrm{g})$ is a semi-simple Lie algebra, and hence we may assume that $g$ is semisimple. If there exists a non-constant relative invariant $f(x)$ satisfying $f(\rho(g) x)=\chi(g) f(x)$, then the infinitesimal character $\delta \chi$ of $\chi$ is not identically zero since $G$ is connected. Hence $\delta x: \mathfrak{g} \rightarrow \mathfrak{g l}(1)$ is an onto map and its kernel Ker $\delta \chi$ is an ideal of $g$ of codimension one. Since $g$ is semisimple, this is a contradiction.

(2) Assume that there exists a relative invariant $f(x)$ corresponding to $\chi$. Then by Theorem 1 in $\S 1, \delta \chi=c \operatorname{tr}_{V}$ for some $c \in C$ where $\operatorname{tr}_{V}$ is 
the trace of $d \rho$ in $V$, and hence $\left.\delta \chi\right|_{g_{x_{0}}} \neq 0$. In particular, we have $\left.\chi\right|_{a_{x_{0}}}$ $\neq 1$. This is a contradiction in view of Proposition 19.

(3) In this case $\operatorname{tr}_{V}$ is not identically zero and $\operatorname{det} \rho\left(G_{x_{0}}\right)$ is a finite group. Hence some power $(\operatorname{det} \rho)^{\ell}$ satisfies the condition $(\operatorname{det} \rho)^{\ell} \neq 1$, and $\left.(\operatorname{det} \rho)^{\ell}\right|_{G_{x_{0}}}=1$. By Proposition 19, there exists a relative invariant corresponding to $(\operatorname{det} \rho)^{\ell}$.

Q.E.D.

Remark 21. Let $(G, \rho, V)$ be an irreducible P. V. and let $G_{x_{0}}$ be a generic isotropy subgroup. Let $S L^{ \pm}(V)$ (resp. $S L(V)$ ) be the group of all non-singular transformations of $V$ of determinant \pm 1 (resp. 1). If $(G, \rho, V)$ is regular, then we have $\rho\left(G_{x_{0}}\right) \subset S L^{ \pm}(V)$ by Propositions 8 and 19. In general, if $\rho(G) \not \subset S L(V)$, then $\rho\left(G_{x_{0}}\right) \subset S L^{ \pm}(V)$ (resp. $\rho\left(G_{x_{0}}\right) \subset$ $S L(V)$ ) if and only if there exists a relative invariant of degree $2 n$ (resp. $n$ ) corresponding to the character $\operatorname{det} \rho(g)^{2}$ (resp. $\operatorname{det} \rho(g)$ ) where $n=\operatorname{dim} V . \quad$ (Note that since $G$ is connected, $\rho(G) \not \subset S L(V)$ implies that $d \rho(\mathfrak{g}) \not \subset \mathfrak{g}(V))$. In particular, if $\operatorname{dim} G=\operatorname{dim} V$, then $\rho\left(G_{x_{0}}\right)$ is a finite group contained in $S L(V)$ by Proposition 16.

Proposition 22 (D. Luna). Let $G$ be a reductive algebraic group which acts on a smooth affine variety $X$. For $x \in X$, let $G_{x}$ be the isotropy subgroup of $G$ at $x$. Assume that, for any point $x$ in $X$, there exists a non-degenerate symmetric $G_{x}$-invariant bilinear form on the tangent space at $x$. Then there exists an open dense subset of $X$ which consists of closed G-orbits in $X$. In pprticular, an open orbit in $X$ is closed.

Proof. See ([21]).

Corollary 23 (J. Dorfmeister). Let $(G, \rho, V)$ be a regular P.V. with a reductive algebraic group $G$ where $\rho$ is not necessarily irreducible. Then its generic isotropy subgroup is also reductive.

Proof. Let $f(x)$ be a relative invariant satisfying $H_{f}(x) \neq 0$. We may assume that $f(x)$ is a polynomial, and hence its Hessian $H_{f}(x)$ is also a polynomial. Put $X=\left\{x \in V \mid H_{f}(x) \neq 0\right\}$. Then $X$ is a smooth affine variety and, for each $x \in X, B_{x}(u, u)=\sum_{i, j} \frac{\partial^{2} f}{\partial x_{i} \partial x_{j}}(x) u_{i} v_{j}$ is a nondegenerate symmetric $G_{x}$-invariant bilinear form on the tangent space of $X$ at $x$ (see the proof of Proposition 8). Since $H_{f}(x)$ is also a relative invariant, the open $G$-orbit in $V$ is contained in $X$. It is dense in 
$X$, and closed by Proposition 22; hence it coincides with $X$. Since $G$ is reductive and the open orbit is affine, the generic isotropy subgroup is reductive.

Q.E.D.

Finally, we shall prove the converse of Corollary 23.

Let $(G, \rho, V)$ be a $\mathrm{P}$. V. with a reductive algebraic group $G$, where $\rho$ is not necessarily irreducible. Let $\left(G, \rho^{*}, V^{*}\right)$ be the dual P. V. of $(G, \rho, V)$, i.e., $\rho^{*}$ is the contragredient representation of $\rho$ on the dual vector space $V^{*}$ of $V$. Choosing a basis of $V$, we may identify $V$ with $\boldsymbol{C}^{n}(n=\operatorname{dim} V)$ and $V^{*}$ with $\boldsymbol{C}^{n}$ by the inner product $\langle x, y\rangle=\sum_{i=1}^{n} x_{i} y_{i}$. In this case we may assume that $G \subset G L(n)$. It can be proved that a reductive algebraic group $G$ is a Zariski closure of a maximal compact subgroup $K$. Moreover, by choosing a suitable basis of $V$, we have $K \subset U(n)$ where $U(n)$ is the group of all $n \times n$ unitary matrices, i.e., $U(n)=\left\{\left.g \in G L(n)\right|^{t} g \bar{g}=I_{n}\right\}$. Then we have $\rho^{*}(g) y={ }^{t} g^{-1} y=\bar{g} y$ for $g \in K$, $y \in C^{n} \simeq V^{*}$. Let $f(x)$ be any relative invariant polynomial of $(G, \rho, V)$ corresponding to a character $\chi$. Put $f^{*}(y)=\overline{f(\bar{y})}$ where ${ }^{-}$is the complex conjugate $\left(y \in C^{n} \simeq V^{*}\right)$. Then for each $g \in K$, we have $f^{*}\left(\rho^{*}(g) y\right)=$ $f^{*}(\bar{g} y)=\overline{f(g \bar{y})}=\overline{\chi(g) f(\bar{y})}=\chi^{-1}(g) f^{*}(y) \quad\left(y \in C^{n} \simeq V^{*}\right)$. Note that $|\chi(g)|=$ 1 for $g \in K \subset U(n)$. Since $G$ is the Zariski closure of $K$, we have $f^{*}\left(\rho^{*}(g) y\right)=\chi^{-1}(g) f^{*}(y)$ for any $g \in G, y \in V^{*}$, i.e., $f^{*}$ is a relative invariant polynomial of $\left(G, \rho^{*}, V^{*}\right)$ corresponding to $\chi^{-1}$. Put

$$
f(x)^{m}=\sum_{i_{1}+\cdots+i_{n}=r m} a_{i_{1}, \ldots, i_{n}}^{(m)} x_{1}^{i_{1}} \cdots x_{n}^{i_{n}}
$$

where $r=\operatorname{deg} f=\operatorname{deg} f^{*}$. Then we have

$$
f^{*}(y)^{m}=\sum_{i_{1}+\cdots+i_{n}=r m} \overline{a_{i_{1}}^{(m)}, \ldots, i_{n}} y_{1}^{i_{1}} \cdots y_{n}^{i_{n}}
$$

and

$$
f^{*}\left(\operatorname{grad}_{x}\right)^{m} f(x)^{m}=\sum_{i_{1}+\cdots+i_{n}=r m}\left|\alpha_{i_{1}, \cdots, i_{n}}^{(m)}\right|^{2} \cdot\left(i_{1} !\right) \cdots\left(i_{n} !\right)
$$

where

$$
f^{*}\left(\operatorname{grad}_{x}\right)^{m}=\sum_{i_{1}+\cdots+i_{n}=r m} \overline{a_{i_{1}, \cdots, i_{n}}^{(m)}}\left(\frac{\partial}{\partial x_{1}}\right)^{i_{1}} \cdots\left(\frac{\partial}{\partial x_{n}}\right)^{i_{n}}
$$

and $m$ is any non-negative integer. By choosing a suitable basis of $V$, we may assume that $f((1,0, \cdots, 0)) \neq 0$, i.e., $a=a_{r 0, \cdots, 0} \neq 0$. Then we haev $f((1,0, \ldots, 0))^{m}=a_{r m, 0, \cdots, 0}=a^{m}$. Since $\left(f^{*}\left(\operatorname{grad}_{x}\right) f^{m}\right)(g x)=f^{*}\left(\operatorname{grad}_{g x}\right)$. 
$f^{m}(g x)=\chi(g)^{m-1} \cdot f^{*}\left(\operatorname{grad}_{x}\right) f(x)^{m}, f^{*}\left(\operatorname{grad}_{x}\right) f(x)^{m}$ is a relative invariant corresponding to $\chi^{m-1}$, and hence there exists a constant $b(m)$ satisfying $f^{*}\left(\operatorname{grad}_{x}\right) f(x)^{m}=b(m) f(x)^{m-1}$. Clearly $b(m)$ is a polynomial in $m$ with degree $\leq r$. Put $r^{\prime}=\operatorname{deg} b(m)$. Then there exists a constant $C$ satisfying $|b(m)| \leq C m^{r^{\prime}}$ for any $m \geq 1$. Hence we have

$$
\begin{gathered}
C^{m} \cdot(m !)^{r^{\prime}} \geq|b(m) b(m-1) \cdots b(1)|=\left|f^{*}\left(\operatorname{grad}_{x}\right)^{m} f(x)^{m}\right| \\
\left.=\sum_{i_{1}+\cdots+i_{n}=r m}\left|a_{i_{1}, \cdots, i_{n}}^{(m)}\right|^{2} \cdot\left(i_{1} !\right)\right\} \cdots\left(i_{n} !\right) \geq|a|^{2 m} \cdot(r m) !
\end{gathered}
$$

for any $m \geq 1$. Since there exists a positive number $m_{0}$ satisfying $m$ ! $>C^{m} /|a|^{2 m}$ for any $m \geq m_{0}$, if $r^{\prime}<r$, we have $(m !)^{r} \geq(m !)^{r^{\prime+1}} \geq(r m)$ ! for any $m \geq m_{0}$. This is a contradiction for $r \geq 2$. If $r=1$ and $r^{\prime}=$ 0 , we have $C^{m} \geq|a|^{2 m} \cdot m$ ! for any $m \geq 0$ and again a contradiction. Hence we have $\operatorname{deg} b(m)=r\left(=\operatorname{deg} f=\operatorname{deg} f^{*}\right)$. Since

$$
\begin{aligned}
f^{*}\left(\operatorname{grad}_{x}\right) f(x)^{m} & =m^{r} \cdot f^{*}(\operatorname{grad} f(x)) \cdot f(x)^{m-r}+(\text { lower term in } m) \\
& =b(m) f(x)^{m-1}=m^{r} b_{0} f(x)^{m-1}+(\text { lower term in } m),
\end{aligned}
$$

we have $f^{*}(\operatorname{grad} f(x)) \cdot f(x)^{m-r}=b_{0} f(x)^{m-1}$, i.e., $f^{*}(\operatorname{grad} \log f(x))=b_{0} / f(x)$ where $b_{0} \neq 0$ since $\operatorname{deg} b(m)=r$. Therefore, we obtain the following proposition.

Proposition 24. Let $(G, \rho, V)$ be a $P$. V. with a reductive algebraic group $G$ where $\rho$ is not necessarily irreducible. Assume that there exists a relative invariant polynomial corresponding to a character $\chi$. Then there exists a relative invariant polynomial $f^{*}(y)$ of $\left(G, \rho^{*}, V^{*}\right)$ corresponding to $\chi^{-1}$, where $\rho^{*}$ is the contragredient representation of $\rho$ on the dual vector space $V^{*}$ of $V$. Moreover, we have $f^{*}(\operatorname{grad} \log f(x)) \neq 0$ for $x \in V-S$.

Now we are ready to prove the following proposition.

Proposition 25. Let $(G, \rho, V)$ be a $P$. V. with a reductive algebraic group $G$ where $\rho$ is not necessarily irreducible. Assume that a generic isotropy subgroup $G_{x_{0}}\left(x_{0} \in V-S\right)$ is also reductive. Then it is a regular P. V.

Proof. It is well known that the quotient $G / G_{x_{0}} \approx V-S$ of reductive algebraic group $G$ is an affine variety if and only if $G_{x_{0}}$ is reductive (see [8]). Since $V-S$ is affine if and only if $S$ is a hypersurface, in our case the singular set $S$ is a hypersurface. Let $f(x)$ be a relative 
invariant polynomial satisfying $S=\{x \in V \mid f(x)=0\}$. Then by Proposition 24 , there exists a relative invariant polynomial $f^{*}$ of $\left(G, \rho^{*}, V^{*}\right)$ corresponding to $f(x)$. Moreover, the singular set $S^{*}$ of $\left(G, \rho^{*}, V^{*}\right)$ is also a hypersurface defined by $f^{*}$, i.e., $S^{*}=\left\{y \in V^{*} \mid f^{*}(y)=0\right\}$. Since $f^{*}(\operatorname{grad} \log f(x)) \neq 0$ for $x \in V-S$ by Proposition 24, we have $\operatorname{grad} \log f(V-S)=V^{*}-S^{*}$, i.e., $\operatorname{grad} \log f$ is generically surjective. This shows that $(G, \rho, V)$ is regular by Remark 11 .

Q.E.D.

Remark 26. Let $(G, \rho, V)$ be a $P . V$. with a reductive algebraic group $G$. Then it is regular if and only if its generic isotropy subgroup is reductive, and hence the regularity is invariant under the castling transform (see Proposition 9 in $\S 2$ ). Since its generic isotropy subgroup is reductive if and only if the singular set $S$ is a hypersurface, a reductive $P . V$. is regular if and only if its singular set is a hypersurface. However, if $G$ is not reductive, it is false. For example, put $G=$ $\left\{\left(\begin{array}{ll}1 & b \\ & a\end{array}\right) \mid a, b \in C, a \neq 0\right\}, V=C^{2}, \rho\left(\begin{array}{ll}1 & b \\ & a\end{array}\right)\left(\begin{array}{l}x \\ y\end{array}\right)=\left(\begin{array}{c}x+b y \\ a y\end{array}\right)$. Then a triplet $(G, \rho, V)$ is a P.V. and its singular set is a hypersurface defined by $y=0$. But it is not regular. Conversely, it is an open problem that the singular set of any regular P.V. is a hypersurface or not.

\section{§. The prehomogeneity and relative invariants of reduced triplets obtained in $\S 3$}

In this section we shall investigate the reduced triplets obtained in Theorem 24 in $\S 3$, especially we shall determine their prehomogeneity.

$$
\left(G \times G L(m), \rho \otimes \Lambda_{1}, V(n) \otimes V(m)\right)
$$

where $\rho$ is an $n$-dimensional irreducible representation of a connected semi-simple algebraic group $G(\neq S L(n))$ with $m \geqq n \geqq 2$. This is a trivial P.V. (See Definition 5 in $\S 3$ ). We may identify $V(n) \otimes V(m)$ with the totality of $n \times m$ matrices $M(n, m)$. We may assume that $G \subset S L(n)$. Then $\left(\rho \otimes \Lambda_{1}\right)\left(g_{1}, g_{2}\right) X=g_{1} X^{t} g_{2}$ for $g_{1} \in G, g_{2} \in G L(m)$, and $X \in M(n, m)$. If $m=n$, then the isotropy subgroup at $I_{n}$ is $\left\{\left(g_{1},{ }^{t} g_{1}^{-1}\right) \mid g_{1} \in G\right\} \cong G$ and hence it is a regular P.V. by Proposition 25 in $\S 4$. The relative invariant is $\operatorname{det} X$. If $m>n$, the generic isotropy subgroup $G_{x_{0}}$ at $x_{0}=\left[I_{n} O_{n, m-n}\right]$ where $O_{n, m-n}$ is the $n \times(m-n)$ zero matrix, is the totality of $\left(g_{1}, g_{2}\right)$ of the form 


$$
g_{2}=\left(\begin{array}{c|c}
{ }^{t} g_{1}^{-1} & 0 \\
\hline * & *
\end{array}\right),
$$

and hence $G_{x_{0}}$ is isomorphic to the semi-direct product of $(G \times G L(m-n)$ ) and the $n(m-n)$-dimensional vector group $\left(G_{a}\right)^{n(m-n)}$ i.e. $G_{x_{0}} \cong(G \times$ $G L((m-n)) \cdot\left(G_{a}\right)^{n(m-n)}$ where $\left(G_{a}\right)$ denotes the one-dimensional additive group: $G_{a} \cong C$. By (2) in Proposition 20 in $\S 4$, it is not regular. There are no relative invariants.

$$
\begin{aligned}
& \left(S L(n) \times G L(m), \Lambda_{1} \otimes \Lambda_{1}, \quad V(n) \otimes V(m)\right) \\
& \quad(m / 2 \geqq n \geqq 1 \text { or } n=m \geqq 1)
\end{aligned}
$$

This is also a trivial P.V. Similarly it is regular if $n=m$ and not regular if $n<m$. (1) and (2) are essentially of the same type. The reason why we separated (2) from (1) is only to avoid the triplets $(S L(n) \times G L(m)$, $\left.\Lambda_{1} \otimes \Lambda_{1}, V(n) \otimes V(m)\right)(m>n>m / 2)$ which is not reduced.

Proposition 1. A trivial P.V., $\left(G \times G L(m), \rho \otimes \Lambda_{1}, V(n) \otimes V(m)\right)$ $(n \leq m)$ is regular if and only if $m=n$.

$$
(G L(1) \times G, \square \otimes \text { adjoint rep., } V(1) \otimes V(n))
$$

where $G$ is an almost simple algebraic group of dimension $n \geqq 3$.

Let $g$ be the Lie algebra of $G$. We may assume that $G \subset G L(n)$ and $\mathfrak{g} \subset \mathfrak{g l}(n)$. Then the adjoint representation $A d$ is given by $A d(g) X=g X g^{-1}$ for any $g \in G, X \in \mathfrak{g}$. Put $\mathfrak{g}(X)=\left\{Y \in \mathfrak{g} \mid \operatorname{ad}(X)^{n} Y=0\right.$ for some $\left.n>0\right\}$ for $X \in \mathfrak{g}$. An element $X$ in $\mathfrak{g}$ is called a regular element if $\operatorname{dim} \mathfrak{g}(X)$ is minimum. If $X_{0}$ is a regular element, then $\mathfrak{h}=\mathfrak{g}\left(X_{0}\right)$ is a Cartan subalgebra. Note that $\operatorname{dim} g(X)$ is the multiplicity of the eigenvalue 0 of $\operatorname{ad}(X)$. Let $A_{1}, \cdots, A_{n}$ be a basis of $\mathrm{g}$ and $\operatorname{put} \operatorname{det}(t I-\operatorname{ad}(X))=t^{n}+\varphi_{1}\left(x_{1}, \cdots, x_{n}\right) t^{n-1}$ $+\cdots+\varphi_{n-\ell}\left(x_{1}, \cdots, x_{n}\right) t^{\ell}(\ell \geqq 1)$ for $X=\sum_{i=1}^{n} x_{i} A_{i} \in \mathfrak{g}$, where $\ell=$ rank $g$ $=\operatorname{dim} \mathfrak{h}$. Then the totality of non-regular elements is a hypersurface $S$ given by $S=\left\{X=\sum_{i=1}^{n} x_{i} A_{1} \in \mathfrak{g} \mid \varphi_{n-\ell}\left(x_{1}, \cdots, x_{n}\right)=0\right\}$. Since $\mathrm{g}\left(g X g^{-1}\right)=$ $g \mathfrak{g}(X) g^{-1}$, this hypersurface is $G$-invariant, and hence if this triplet is a P.V., then a generic point must be a regular element. Let $X_{0}$ be a regular element. Let $\tilde{\mathfrak{g}}_{X_{0}}$ be the isotropy subalgebra $\tilde{\mathfrak{g}}_{X_{0}}=\{(c, X) \in \mathfrak{g r}(1)$ $\left.\oplus \mathfrak{g} \mid c X_{0}+\left[X, X_{0}\right]=0\right\}$ at $X_{0}$. Then it is a P.V. if and only if $\operatorname{dim} \tilde{\mathrm{g}}_{X_{0}}$ $=\operatorname{dim}(G L(1) \times G)-\operatorname{dim} \mathfrak{g}=1$. Since $\mathfrak{h}=\mathfrak{g}\left(X_{0}\right)$ is a Cartan subalgebra and hence commutative, we have $\mathfrak{h}=\left\{(0, X) \in \mathfrak{g} l(1) \oplus \mathfrak{g} \mid\left[X, X_{0}\right]=0\right\} \subset \tilde{\mathfrak{g}}_{X_{0}}$. This shows that $\operatorname{dim} \tilde{g}_{x_{0}} \geqq \operatorname{dim} \mathfrak{h}=\operatorname{rankg}$ and hence it is not a $\mathrm{P}$.V. 
if rank $\mathfrak{g}>1$. Assume that rank $\mathfrak{g}=1$. Then $\mathfrak{g}=\mathfrak{g l}(2)(=\mathfrak{g p}(1)=\mathfrak{D}(3))$ (See Example 45 in $\S 1$ ), and it is equivalent to $\left(G L(2), 2 \Lambda_{1}, V(3)\right.$ ). The representation space can be considered as the space of all binary quadratic forms $F_{x}(u, v)=x_{1} u^{2}+x_{2} u v+x_{3} v^{2}$ with $x=\left(x_{1}, x_{2}, x_{3}\right) \in C^{3}$. The action $\rho=2 \Lambda_{1}$ of $G L(2)$ is given by $\left(\rho(g) F_{x}\right)(u, v)=F_{x}((u, v) g)$ for $g \in G L(2)$. Since the isotropy subgroup $G_{X_{0}}$ at $X_{0}=u v$ is

$$
O(2)=\left\{\left(\begin{array}{ll}
\alpha & 0 \\
0 & \alpha^{-1}
\end{array}\right) \mid \alpha \neq 0\right\},
$$

it is a regular P.V. by Proposition 25 in $\S 4$. The relative invariant $f(x)$ is a discriminant of $F_{x}$ i.e. $f(x)=x_{2}^{2}-4 x_{1} x_{3}$ for $x=F_{x}$.

PRoposition 2. The composition of scalar multiplications and the adjoint representation of an almost simple algebraic group $G$ is prehomogeneous if and only if $\operatorname{rank} G=1$. If $\operatorname{rank} G=1$, it is a regular P.V. equivalent to $\left(G L(2), 2 \Lambda_{1}, V(3)\right)$.

$$
\left(G L(n), 2 \Lambda_{1}, V\left(\frac{1}{2} n(n+1)\right)\right) \quad(n \geqq 3)
$$

Put $V=\left\{\left.X \in M(n, C)\right|^{t} X=X\right\}$. Then the action $\rho=2 \Lambda_{1}$ of $G L(n)$ is given by $\rho(A) X=A X^{t} A$ for $A \in G L(n), X \in V$. The isotropy subgroup at the unit matrix $I_{n}$ is by definition the orthogonal group $O(n)=$ $\left\{A \in G L(n) \mid A^{t} A=1\right\}$ and since $\operatorname{dim} O(n)=\frac{1}{2} n(n-1)=\operatorname{dim} G L(n)-$ $\operatorname{dim} V\left(\frac{1}{2} n(n+1)\right)$, it is a P.V. By Proposition 25 in $\S 4$, it is regular and the relative invariant is given by $\operatorname{det} X$ for $X \in V$.

Proposition 3. A triplet $\left(G L(n), 2 \Lambda_{1}, V\left(\frac{1}{2} n(n+1)\right)\right)$ is a regular $P . V$. and its generic isotropy subgroup is the orthogonal group $O(n)$.

$$
\left(G L(n), \Lambda_{2}, V\left(\frac{1}{2} n(n-1)\right)\right) \quad(n \geqq 5)
$$

Put $V=\left\{X \in M(n, C) \mid{ }^{t} X=-X\right\}$. Then the action $\rho=\Lambda_{2}$ of $G L(n)$ is given by $\rho(A) X=A X^{t} A$ for $A \in G L(n), X \in V$. Assume that $n$ is even, i.e., $n=2 m$. Then the isotropy subgroup at

$$
J=\left(\begin{array}{c|c}
0 & I_{m} \\
\hline-I_{m} & 0
\end{array}\right)
$$

is by definition the symplectic group $S p(m)=\left\{A \in G L(2 m) \mid{ }^{t} A J A=J\right\}$ since ${ }^{t} A J A=J$ if and only if $A J^{t} A=A J\left({ }^{t} A J A\right) A^{-1} J^{-1}=A J(J) A^{-1} J^{-1}=-J^{-1}$ $=J$. Since $\operatorname{dim} S p(m)=m(2 m+1)=\operatorname{dim} G L(2 m)-\operatorname{dim} V(m(2 m-1))$, it is a P.V. By Proposition 25 in $\S 4$, it is regular and the relative in- 
variant is the Pfaffian of a skew-symmetric matrix $X$ in $V$. Next assume that $n$ is odd i.e. $n=2 m+1$. Put

$$
x_{0}=\left(\begin{array}{c|c}
J & 0 \\
\hline 0 & 0
\end{array}\right) \text {. }
$$

Since

$$
\begin{aligned}
& \left(\begin{array}{l|l}
A & B \\
\hline C & D
\end{array}\right)\left(\begin{array}{c|c}
J & 0 \\
\hline 0 & 0
\end{array}\right)^{t}\left(\begin{array}{l|l}
A & B \\
\hline C & D
\end{array}\right)=\left(\begin{array}{c|c}
A J^{t} A & A J^{t} C \\
\hline C J^{t} A & C J^{t} C
\end{array}\right)=\left(\begin{array}{c|c}
J & 0 \\
\hline 0 & 0
\end{array}\right) \\
& \text { for }\left(\begin{array}{c|c}
A & B \\
\hline C & D
\end{array}\right) \in G_{x_{0}},
\end{aligned}
$$

we have

$$
G_{x_{0}}=\left\{\left(\frac{A}{0} \mid \frac{B}{D}\right) \mid A J^{t} A=J\right\} \cong(S p(m) \times G L(1)) \cdot\left(G_{a}\right)^{2 m} .
$$

Since

$$
\begin{aligned}
\operatorname{dim}( & S p(m) \times G L(1)) \cdot\left(G_{a}\right)^{2 m} \\
& =(m+1)(2 m+1) \\
& =\operatorname{dim} G L(2 m+1)-\operatorname{dim} V(m(2 m+1)),
\end{aligned}
$$

it is a P.V. By (2) in Proposition 20 in $\S 4$, there is no relative invariant and hence it is not regular. Thus we have the following proposition.

Proposition 4. A triplet $\left(G L(2 m), \Lambda_{2}, V(m(2 m-1))\right)$ is a regular $P . V$. and its generic isotropy subgroup is the symplectic group $S p(m)$. The relative invariant is the Pfaffian and hence of degree $m$.

Proposition 5. A triplet $\left(G L(2 m+1), \Lambda_{2}, V(m(2 m+1))\right)$ is a P.V. There is no relative invariant and hence not regular. The generic isotropy subgroup is isomorphic to the semi-direct product $(S p(m) \times G L(1)) \cdot\left(G_{a}\right)^{2 m}$.

$$
\left(G L(2), 3 \Lambda_{1}, V(4)\right)
$$

The representation space can be identified with the space of all binary cubic forms $F_{x}(u, v)=x_{1} u^{3}+x_{2} u^{2} v+x_{3} u v^{2}+x_{4} v^{3}$ with $x=\left(x_{1}, x_{2}, x_{3}, x_{4}\right) \in C^{4}$. Then the action $\rho=3 \Lambda_{1}$ of $G L(2)$ is given by $\left(\rho(g) F_{x}\right)(u, v)=F_{x}((u, v) g)$ (See (6) in Example 24 in $\S 1$ ). For each binary cubic form $F_{x}=F_{x}(u, v)$ $=x_{1} u^{3}+x_{2} u^{2} v+x_{3} u v^{2}+x_{4} v^{3}$, let $F_{x}^{\prime}$ be the binary quadratic form defined by 


$$
\begin{aligned}
F_{x}^{\prime} & =\frac{1}{4} \operatorname{det}\left(\begin{array}{cc}
\frac{\partial^{2} F}{\partial u \partial u} & \frac{\partial^{2} F}{\partial u \partial v} \\
\frac{\partial^{2} F}{\partial v \partial u} & \frac{\partial^{2} F}{\partial v \partial v}
\end{array}\right)=\operatorname{det}\left(\begin{array}{cc}
3 x_{1} u+x_{2} v, & x_{2} u+x_{3} v \\
x_{2} u+x_{3} v, & x_{3} u+3 x_{4} v
\end{array}\right) \\
& =\left(3 x_{1} x_{3}-x_{2}^{2}\right) u^{2}+\left(9 x_{1} x_{4}-x_{2} x_{3}\right) u v+\left(3 x_{2} x_{4}-x_{3}^{2}\right) v^{2} .
\end{aligned}
$$

Then the correspondence $F_{x} \mapsto F_{x}^{\prime}$ gives a generically surjective $G L(2)-$ equivariant morphism $\varphi$ of $V$ into $V^{\prime}$ where $V$ (resp. $V^{\prime}$ ) denotes the space of all binary cubic (resp. binary quadratic) forms. As we have seen in (3) just before Proposition 2, a triplet $\left(G L(2), 2 \Lambda_{1}, V^{\prime}\right)$ is a regular P.V. and the generic isotropy subgroup $G_{X_{0}^{\prime}}$ at $X_{0}^{\prime}=9 u v$ is given by $G_{X_{0}^{\prime}}=O(2)$ $=\left\{\left(\begin{array}{cc}\alpha & 0 \\ 0 & \alpha^{-1}\end{array}\right) \mid \alpha \neq 0\right\}$. The fiber $\varphi^{-1}\left(X_{0}^{\prime}\right)$ of $\varphi$ at $X_{0}^{\prime}$ is by definition $\varphi^{-1}\left(X_{0}^{\prime}\right)$ $=\left\{F_{x}(u, v) \in V \mid 3 x_{1} x_{3}-x_{2}^{2}=3 x_{2} x_{4}-x_{3}^{2}=0,9 x_{1} x_{4}-x_{2} x_{3}=9\right\}=\left\{F_{x}(u, v)=\right.$ $\left.x_{1} u^{3}+x_{1}^{-1} v^{3} \mid x_{1} \neq 0\right\}$. Since an element $\left(\begin{array}{cc}\alpha & 0 \\ 0 & \alpha^{-1}\end{array}\right)$ of $G_{X_{0}^{\prime}}$ acts on the fiber $\varphi^{-1}\left(X_{0}^{\prime}\right)$ such as $x_{1} u^{3}+x_{1}^{-1} v^{3} \mapsto\left(\alpha x_{1}\right) u^{3}+\left(\alpha x_{1}\right)^{-1} v^{3}, \varphi^{-1}\left(X_{0}^{\prime}\right)$ is $G_{X_{0}^{\prime}}$-homogeneous, and hence by Lemma 5 in $\S 2$, a triplet $\left(G L(2), 3 \Lambda_{1}, V(4)\right)$ is a P.V. We shall determine the isotropy subgroup $G_{X_{0}}$ at $X_{0}=u^{3}+v^{3}$ in $\varphi^{-1}\left(X_{0}^{\prime}\right)$. If we identify $V$ with $C^{4}$ by an isomorphism $F_{x}(u, v) \mapsto x={ }^{t}\left(x_{1}, x_{2}, x_{3}, x_{4}\right) \in C^{4}$, we have

$$
\begin{aligned}
& \rho(g) x=\left(\begin{array}{cccc}
\alpha^{3} & \alpha^{2} \beta & \alpha \beta^{2} & \beta^{3} \\
3 \alpha^{2} \gamma & \alpha^{2} \delta+2 \alpha \beta \gamma & 2 \alpha \beta \delta+\gamma \beta^{2} & 3 \beta^{2} \delta \\
3 \alpha \gamma^{2} & 2 \alpha \gamma \delta+\gamma^{2} \beta & \alpha \delta^{2}+2 \beta \gamma \delta & 3 \beta \delta^{2} \\
\gamma^{3} & \gamma^{2} \delta & \gamma \delta^{2} & \delta^{3}
\end{array}\right)\left(\begin{array}{l}
x_{1} \\
x_{3} \\
x_{3} \\
x_{4}
\end{array}\right) \\
& \text { for } g=\left(\begin{array}{ll}
\alpha & \beta \\
\gamma & \delta
\end{array}\right) \in G L(2)
\end{aligned}
$$

and hence

$$
\begin{aligned}
G_{X_{0}} & =\left\{\left(\begin{array}{ll}
\alpha & \beta \\
\gamma & \delta
\end{array}\right) \in G L(2) \mid \alpha^{3}+\beta^{3}=\gamma^{3}+\delta^{3}=1, \alpha^{2} \gamma+\beta^{2} \delta=\alpha \gamma^{2}+\beta \delta^{2}=0\right\} \\
& =\left\{\left(\begin{array}{ll}
\alpha & 0 \\
0 & \delta
\end{array}\right) \mid \alpha^{3}=\delta^{3}=1\right\} \cup\left\{\left(\begin{array}{ll}
0 & \beta \\
\gamma & 0
\end{array}\right) \mid \beta^{3}=\gamma^{3}=1\right\} .
\end{aligned}
$$

From (5.2), the kernel of $\rho$ is the central cyclic group $\mathscr{Z}$ of order 3:

$$
\mathscr{Z}=\left\{\left(\begin{array}{cc}
\omega & 0 \\
0 & \omega
\end{array}\right) \mid \omega^{3}=1\right\} \text {. }
$$

Thus the isotropy subgroup $G_{X_{0}}$ is a finite group of order 18 and 
its image $\rho\left(G_{X_{0}}\right) \cong G_{X_{0}} / \mathscr{Z}$ is a finite group of order 6 . In particular, it is a regular P.V. by Proposition 25 in $\S 4$. Since $\varphi$ is equivariant, the discriminant of $F_{x}^{\prime}$ i.e.

$$
\begin{aligned}
& \left(9 x_{1} x_{4}-x_{2} x_{3}\right)^{2}-4\left(3 x_{1} x_{3}-x_{2}^{2}\right)\left(3 x_{2} x_{4}-x_{3}^{2}\right) \\
& \quad=-3\left(x_{2}^{2} x_{3}^{2}+18 x_{1} x_{2} x_{3} x_{4}-4 x_{1} x_{3}^{3}-4 x_{2}^{3} x_{4}-27 x_{1}^{2} x_{4}^{2}\right)
\end{aligned}
$$

is a relative invariant, and its character is $(\operatorname{det} g)^{6}$ for $g \in G L(2)$. Note that $P(x)=\left(x_{2}^{2} x_{3}^{2}+18 x_{1} x_{2} x_{3} x_{4}-4 x_{1} x_{3}^{3}-4 x_{2}^{3} x_{4}-27 x_{1}^{2} x_{4}^{2}\right)$ is the discriminant of the binary cubic form $F_{x}(u, v)$.

Let $\chi$ be any rational character of $G L(2)$ i.e. $\chi(g)=(\operatorname{det} g)^{m}$ for some integer $m$.

Since the restriction $\left.\chi\right|_{G_{X_{0}}}$ of $\chi$ to $G_{X_{0}}$ is identity if and only if $(\alpha \delta)^{m}=(-\beta \gamma)^{m}=1$ for any $\alpha, \beta, \gamma, \delta$ satisfying $\alpha^{3}=\beta^{3}=\gamma^{3}=\delta^{3}=1$ i.e., $m=6 n$ for some integer $n$. Hence any relative invariant is of the form $c P(x)^{n}(c \in C, n \in Z)$ by Proposition 19 in $\S 4$. In particular $P(x)$ must be irreducible. The existence of the equivariant polynomial map $\varphi$ of degree 2 is based on the fact that the symmetric product $S^{2}(V)$ of the representation space $V$ of $3 \Lambda_{1}$ decomposes into the dirəct sum $S^{2}(V)=$ $V_{1} \oplus V_{2}$ as a representation space of $G L(n)$ where $V_{1}$ (resp. $V_{2}$ ) is corresponding $6 \Lambda_{1}$ (resp. $2 \Lambda_{1}+2 \Lambda_{2}$ ) i.e.

$$
S^{2}\left(3 \Lambda_{1}\right)=6 \Lambda_{1} \oplus\left(2 \Lambda_{1}+2 \Lambda_{2}\right)
$$

or

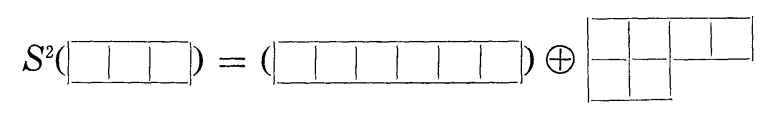

by the Young diagram (See $\S 1$ ). For $n=2$, we have $\left(S L(2), 2 \Lambda_{1}+2 \Lambda_{2}\right.$, $\left.V_{2}\right) \cong\left(S L(2), 2 \Lambda_{1}, V\right)$ and hence we know that $\varphi$ is a map obtained by the composition $V \rightarrow S^{2}(V)=V_{1} \oplus V_{2} \rightarrow V_{2} \cong V^{\prime}$. Since $\operatorname{dim} G L(2)=\operatorname{dim} V$, we can also use Proposition 16 in $\S 4$ to construct a relative invariant. By (6) in Example 30 in $\S 1$, we have

$$
\begin{aligned}
d \rho(A) x= & \left.\begin{array}{cccc}
3 a & b & 0 & 0 \\
3 c & 2 a+d & 2 b & 0 \\
0 & 2 c & a+2 d & 3 b \\
0 & 0 & c & 3 d
\end{array}\right]\left(\begin{array}{l}
x_{1} \\
x_{2} \\
x_{3} \\
x_{4}
\end{array}\right) \\
\text { for } A & =\left(\begin{array}{ll}
a & b \\
c & d
\end{array}\right) \in \operatorname{gr}(2)
\end{aligned}
$$


Note that (5.3) is also obtained from (5.2) by putting

$$
\left(\begin{array}{ll}
\alpha & \beta \\
\gamma & \delta
\end{array}\right)=\left(\begin{array}{ll}
1 & 0 \\
0 & 1
\end{array}\right)+t\left(\begin{array}{ll}
a & b \\
c & d
\end{array}\right)
$$

in (5.2) and taking out the coefficients of the linear term $t$ since

$$
\rho\left(\exp t\left(\begin{array}{ll}
a & b \\
c & d
\end{array}\right)\right)=\exp t d \rho\left(\begin{array}{ll}
a & b \\
c & d
\end{array}\right)
$$

for any $t \in C$. The isotropy subalgebra at $X_{0}=u^{3}+v^{3}$ (i.e. $X_{0}={ }^{t}(1$, $0,0,1)$ ) is 0 from (5.3) and hence it is a regular P.V. by Proposition 16 in $\S 4$. The isotropy subalgebra $g_{X_{0}^{\prime}}$ at $X_{0}^{\prime}=u^{2} v$ (i.e. $X_{0}^{\prime}={ }^{t}(0,1,0,0)$ ) is

$$
g_{X_{0}^{\prime}}=\left\{\left(\begin{array}{rr}
a & 0 \\
0 & -a
\end{array}\right) \in \operatorname{gr}(2)\right\}
$$

and hence the orbit of $X_{0}^{\prime}$ is of codimension one. By Proposition 16 in $\S 4$, there exists an irreducible relative invariant polynomial $f(x)$ of degree $4(=\operatorname{dim} V)$. We shall construct it according to the proof of Proposition 16 in $\S 4$. Let $A_{1}$ (resp. $A_{2}, A_{3}, A_{4}$ ) be the $4 \times 4$ matrix with $a=1$ (resp. $b, c, d=1$ ), all remaining entries zero in (5.3). Then $\left\{A_{1}, \cdots, A_{4}\right\}$ is a basis of $d \rho(g \backslash(2))$ and for $x={ }^{t}\left(x_{1}, x_{2}, x_{3}, x_{4}\right)$, we have

$$
\begin{aligned}
f(x) & =\operatorname{det}\left(A_{1} x, \cdots, A_{4} x\right)=\operatorname{det}\left(\begin{array}{cccc}
3 x_{1} & x_{2} & 0 & 0 \\
2 x_{2} & 2 x_{3} & 3 x_{1} & x_{2} \\
x_{3} & 3 x_{4} & 2 x_{2} & 2 x_{3} \\
0 & 0 & x_{3} & 3 x_{4}
\end{array}\right) \\
& =3\left(x_{2}^{2} x_{3}^{2}+18 x_{1} x_{2} x_{3} x_{4}-4 x_{1} x_{3}^{3}-4 x_{2}^{3} x_{4}-27 x_{1}^{2} x_{4}^{2}\right) .
\end{aligned}
$$

Proposition 6. A triplet $\left(G L(2), 3 \Lambda_{1}, V(4)\right)$ is a regular P.V. and its generic isotropy subgroup is a finite group of order 18 . The representation space can be identified with the space of binary cubic forms $F_{x}(u, v)$ $=x_{1} u^{3}+x_{2} u^{2} v+x_{3} u v^{2}+x_{4} v^{3}\left(\left(x_{1}, x_{2}, x_{3}, x_{4}\right) \in C^{4}\right)$ and then the action $\rho=$ $3 \Lambda_{1}$ of $G L(2)$ is given by $\left(\left(\rho(g) F_{x}\right)(u, v)=F_{x}((u, v) g)\right.$ for $g \in G L(2)$. The relative invariant $f(x)$ is the discriminant of $F_{x}$ i.e. $f(x)=x_{2}^{2} x_{3}^{2}+18 x_{1} x_{2} x_{3} x_{4}$ $-4 x_{1} x_{3}^{3}-4 x_{2}^{3} x_{4}-27 x_{1}^{2} x_{4}^{2}$.

$$
\left(G L(6), \Lambda_{3}, V(20)\right)
$$

Let $V_{1}=\sum_{i=1}^{6} C u_{i}$ be a vector space with a basis $\left\{u_{1}, \cdots, u_{6}\right\}$. Then $G L(6)$ acts on $V_{1}$ as $\left(u_{1}, \cdots, u_{6}\right) \mapsto\left(u_{1}, \cdots, u_{6}\right) g$ for $g \in G L(6)$. In general, 
let $V_{\nu}=\sum_{i_{1}<\cdots<i \nu} C u_{i_{1}} \wedge \cdots \wedge u_{i_{\nu}}$ be the skew-symmetric tensors of rank $\nu$ $(1 \leq \nu \leq 6)$. Then $\operatorname{dim} V_{\nu}=\left(\begin{array}{l}6 \\ \nu\end{array}\right), V_{6}=C \tau$ where $\tau=u_{1} \wedge \cdots \wedge u_{6}$, and the representation space of $\left(G L(6), \Lambda_{3}, V(20)\right)$ can be identified with $V_{3}$ under the induced action of $G L(6)$. Denote by $A$ the Grassmann algebra generated by $V_{1}$ i.e. $A=C+V_{1}+\cdots+V_{6}$, and define the polarization $D_{\nu}: V_{\nu} \rightarrow V_{\nu} \otimes V_{1}$ as a part of the derivation map $D$ from $A$ to a left $A$-module $A \otimes V_{1}$ determined by $D_{1}: V_{1} \rightarrow C \otimes V_{1}$ which is given by $D_{1}(\omega)$ $=1 \otimes \omega$. Namely $D_{\nu}$ is defined by $D_{\nu}\left(u_{i_{1}} \wedge \cdots \wedge u_{i_{\nu}}\right)=\sum_{\mu=1}^{\nu}(-1)^{\nu-\mu} u_{i_{1}} \wedge$ $\cdots \wedge u_{i_{\mu-1}} \wedge u_{i_{\mu+1}} \wedge \cdots \wedge u_{i_{\nu}} \otimes u_{i_{\mu}}$. For each $\eta \in V_{4}$ and $\theta \in V_{3}$, we have $(\eta \otimes 1) \wedge D_{3}(\theta) \in V_{6} \otimes V_{1}=C \tau \otimes V_{1}=\tau \otimes V_{1}$, and hence there exists a bilinear map $L: V_{4} \times V_{3} \rightarrow V_{1}$ satisfying $(\eta \otimes 1) \wedge D_{3}(\theta)=\tau \otimes L(\eta, \theta)$ for any $\eta \in V_{4}, \theta \in V_{3}$. Now for each $\theta \in V_{3}$, define a linear endomorphism $S_{\theta}$ of $V_{1}$ by $S_{\theta}(\omega)=L(\omega \wedge \theta, \theta)$ for $\omega \in V_{1}$. Note that matrix elements of $S_{\theta}$ are quadratic forms of coefficients $x_{i j k}$ of $\theta=\sum x_{i j k} u_{i} \wedge u_{j} \wedge u_{k} \in V_{3}$. For $\theta_{0}=u_{1} \wedge u_{2} \wedge u_{3}+u_{4} \wedge u_{5} \wedge u_{6}$, from $\tau \otimes S_{\theta}(\omega)=((\omega \wedge \theta) \otimes 1) \wedge D_{3}(\theta)$, we have $S_{\theta_{0}}\left(u_{i}\right)=u_{i}(1 \leq i \leq 3)$ and $S_{\theta}\left(u_{i}\right)=-u_{i}(4 \leq i \leq 6)$. Hence we can divide $V_{1}$ into eigenspaces $V_{\theta_{0}}^{ \pm}$of $S_{\theta_{0}}$.

$$
V_{1}=V_{\theta_{0}}^{+}+V_{\theta_{0}} \quad \text { where } V_{\theta_{0}}^{+}=\sum_{i=1}^{3} \boldsymbol{C} u_{i}, V_{\theta_{0}}=\sum_{i=4}^{6} \boldsymbol{C} u_{i} .
$$

On the other hand, for $\omega \in V_{1}, T \in G L(6)$, we have $\tau \otimes S_{T(\theta)} \circ T(\omega)=$ $\tau \otimes L(T(\omega) \wedge T(\theta), T(\theta))=(T(\omega) \wedge T(\theta) \otimes 1) \wedge D_{3} T(\theta)=T\left((\omega \wedge \theta) \otimes 1 \wedge D_{3}(\theta)\right)$ $=T\left(\tau \otimes S_{\theta}(\omega)\right)=(\operatorname{det} T) \tau \otimes T \circ S_{\theta}(\omega)$ and hence $S_{T(\theta)}=(\operatorname{det} T) T \circ S_{\theta} \circ T^{-1}$. Let $G_{\theta_{0}}$ be the isotropy subgroup $\left\{T \in G L(6) \mid T\left(\theta_{0}\right)=\theta_{0}\right\}$ of $G L(6)$. Then we have $S_{\theta_{0}} T\left(u_{1}\right)=(\operatorname{det} T) T\left(u_{1}\right)$ for $T \in G_{\theta_{0}}$. This implies $(\operatorname{det} T)= \pm 1$ since eigenvalues of $S_{\theta_{0}}$ is \pm 1 . If $\operatorname{det} T=1$, then we have $S_{\theta_{0}} T=T S_{\theta_{0}}$ and hence $T\left(V_{\theta_{0}}^{+}\right) \subset V_{\theta_{0}}^{+}$and $T\left(V_{\theta_{0}}^{-}\right) \subset V_{\theta_{0}}^{-}$Therefore $T\left(\theta_{0}\right)=\operatorname{det}\left(\left.T\right|_{V_{\theta_{0}}^{+}}\right) u_{1} \wedge u_{2}$ $\wedge u_{3}+\operatorname{det}\left(\left.T\right|_{V_{\theta_{0}}}\right) u_{4} \wedge u_{5} \wedge u_{6}=\theta_{0}$ and hence $T$ must be in $S L\left(V_{\theta_{0}}^{+}\right) \times \stackrel{S}{S} L\left(V_{\theta_{0}}^{-}\right)$. Conversely it is clear that $S L\left(V_{\theta_{0}}^{+}\right) \times S L\left(V_{\theta_{0}}^{-}\right) \subset G_{\theta_{0}}$. Put

$$
T_{0}=\left(\frac{I_{3}}{I_{3}}\right)
$$

Then $T_{0} \in G_{\theta_{0}}$ and $\operatorname{det} T_{0}=-1$, moreover for any $T$ in $G_{\theta_{0}}$ with $\operatorname{det} T=$ $-1, T T_{0}^{-1}$ is in $S L\left(V_{\theta_{0}}^{+}\right) \times S L\left(V_{\theta_{0}}\right)$. Hence the isotropy subgroup $G_{\theta_{0}}$ is given by

$$
G_{\theta_{0}}=\left\{\left(\begin{array}{c|c}
A & 0 \\
\hline 0 & B
\end{array}\right) \mid A, B \in S L(3)\right\} \times\left\{\left(\begin{array}{c|c}
0 & I_{3} \\
\hline I_{3} & 0
\end{array}\right), I_{6}\right\}
$$




$$
\cong(S L(3) \times S L(3)) \times\{ \pm 1\}
$$

Since $\operatorname{dim} G_{\theta_{0}}=16=\operatorname{dim} G L(6)-\operatorname{dim} V_{3}$, a triplet $\left(G L(6), \Lambda_{3}, V_{3}\right)$ is a P.V. and by Proposition 25 in $\S 4$, it is regular. Hence we obtain the first assertion of the following proposition.

Proposition 7. (1) A triplet $\left(G L(6), \Lambda_{3}, V(20)\right)$ is a regular P.V. and its generic isotropy subgroup is isomorphic to $(S L(3) \times S L(3)) \times\{ \pm 1\}$. (2) trace $S_{\theta}=0$ for any $\theta \in V_{3}$. (3) There exists an irreducible relative invariant polynomial $P$ of degree 4 satisfying $S_{\theta}^{2}=P(\theta) I_{6}$ for any $\theta \in V_{3}$. (4) Let $K$ be a subfield, of $C$, and put $V_{k}=\sum_{i=1}^{6} K u_{i}$. Then $S L\left(V_{k}\right)$ operates transitively on K-rational points of the hypersurface $W=\left\{\theta \in V_{3} \mid P(\theta)=1\right\}$.

Proof. (2) Since eigenvalues of $S_{\theta_{0}}$ is $1,1,1,-1,-1,-1$, we have $\operatorname{trace} S_{\theta_{0}}=1+1+1-1-1-1=0$. For $\theta=T \theta_{0}(\operatorname{det} T \neq 0)$,

$$
\operatorname{trace} S_{\theta}=\operatorname{trace}(\operatorname{det} T) T S_{\theta_{0}} T^{-1}=0 \text {. }
$$

Since the Zariski closure of $\left\{\theta \in V_{3} \mid \theta=T\left(\theta_{0}\right)\right.$, $\left.\operatorname{det} T \neq 0\right\}$ is $V_{3}$, we have our assertion. (3) Since $S_{\theta_{0}}^{2}\left(u_{i}\right)=u_{i}(1 \leq i \leq 6)$, we have $S_{\theta_{0}}^{2}=I_{6}$. For $\theta=T \theta_{0}(\operatorname{det} T \neq 0)$, we have $S_{\theta}^{2}=\left((\operatorname{det} T) T S_{\theta_{0}} T^{-1}\right)^{2}=(\operatorname{det} T)^{2} I_{6}$. Since the Zariski closure of $\left\{\theta \in V_{3} \mid \theta=T\left(\theta_{0}\right)\right.$, det $\left.T \neq 0\right\}$ is $V_{3}$, there exists a polynomial $P$ on $V_{3}$ satisfying $S_{\theta}^{2}=P(\theta) I_{6}$. Clearly $P(\theta)$ is a relative invariant corresponding to $(\operatorname{det} T)^{2}$.

Since $\left.(\operatorname{det} T)^{m}\right|_{G_{0}}=1$ if and only if $m$ is even i.e. $m=2 n(n \in Z)$, $P(\theta)$ is irreducible and any relative invariant is of the form $c P(\theta)^{n} \quad(c \in C$, $n \in Z$ ) by Proposition 19 in $\S 4$. For $T=t I_{6}\left(t \in C^{\times}\right)$, since $(\operatorname{det} T)^{2}=t^{12}$ and $P(T \theta)=t^{3 \operatorname{deg} P} . \quad P(\theta)$, we have $t^{12}=t^{3} \operatorname{deg} P\left(t \in C^{\times}\right)$and hence $\operatorname{deg} P=4$. (4) Let $\theta$ be a $K$-rational point in $W$. Then $S_{\theta}$ is defined over $K$ and $V_{\theta}^{ \pm},_{K}=\frac{1}{2}\left(I_{6} \pm S_{\theta}\right) V_{K}$ is an eigenspace of $S_{\theta}$ corresponding to \pm 1 since $S_{\theta}^{2}$ $=I_{6}$. Note that $V_{k}=V_{\theta, K}^{+}+V_{\bar{\theta}, K}$.

Since $P(\theta) \neq 0$, there exists $T \in G L(6)$ satisfying $\theta=T\left(\theta_{0}\right)$. Put $\tilde{v}_{i}=$ $T u_{i}(1 \leq i \leq 6)$. Then we have $\theta=\tilde{v}_{1} \wedge \tilde{v}_{2} \wedge \tilde{v}_{3}+\tilde{v}_{4} \wedge \tilde{v}_{5} \wedge \tilde{v}_{6}$ and $S_{\theta}\left(\tilde{v}_{i}\right)=$ $(\operatorname{det} T) T S_{\theta_{0}} T^{-1}\left(T \theta_{i}\right)=(\operatorname{det} T) \tilde{v}_{i}(1 \leq i \leq 3), S_{\theta}\left(\tilde{v}_{i}\right)=-(\operatorname{det} T) \tilde{v}_{i}(4 \leq i \leq 6)$. Since $V_{3}=V_{\theta, C}^{+}+V_{\theta, c}^{-}$where $V_{\theta, c}^{ \pm}=\frac{1}{2}\left(1 \pm S_{\theta}\right) V_{3}$, an eigenvalue of $S_{\theta}$ is \pm 1 , and hence $(\operatorname{det} T)= \pm 1$. Therefore by changing indices if necessary, we may assume that $\theta=\tilde{v}_{1} \wedge \tilde{v}_{2} \wedge \tilde{v}_{3}+\tilde{v}_{4} \wedge \tilde{v}_{5} \wedge \tilde{v}_{6}, \quad V_{\theta, \boldsymbol{C}}^{+}=\sum_{i=1}^{3} \boldsymbol{C} \tilde{v}_{i}$ and $V_{\bar{\theta}, C}=\sum_{i=4}^{6} C \tilde{v}_{i}$. There exist $v_{i}^{\prime}(1 \leq i \leq 6)$ in $V_{K}$ and $c_{i} \in C(i=1,2)$ such that $\theta=c_{1} v_{1}^{\prime} \wedge v_{2}^{\prime} \wedge v_{3}^{\prime}+c_{2} v_{4}^{\prime} \wedge v_{5}^{\prime} \wedge u_{6}^{\prime}, V_{\theta, K}^{+}=\sum_{i=1}^{3} K v_{i}^{\prime}$ and $V_{\bar{\theta}, K}^{-}=\sum_{i=4}^{6} K v_{i}^{\prime}$. 
If $c_{1}=0$ or $c_{2}=0$, we have $P(\theta)=0$ by simple calculation. This is a contradiction since $\theta \in W$. Therefore $c_{1} \neq 0, c_{2} \neq 0$ and moreover $c_{1}$ and $c_{2}$ are in $K$ since $\theta$ is $K$-rational.

Put $v_{1}=c_{1} v_{1}^{\prime}, v_{4}=c_{2} v_{4}^{\prime}, v_{i}^{\prime}=v_{\imath}^{\prime}(i \neq 1,4)$. Then we have $\theta=v_{1} \wedge v_{2}$ $\wedge v_{3}+v_{4} \wedge v_{5} \wedge v_{6}, V_{\theta, K}^{+}=\sum_{i=1}^{3} K v_{i}$ and $V_{\bar{\theta}, K}^{-}=\sum_{i=4}^{6} K v_{i}$. Hence there exists $T^{\prime}$ in $G L\left(V_{K}\right)$ satisfying $T^{\prime}\left(u_{i}\right)=v_{i}(1 \leq i \leq 6)$. Since $S_{\theta}=\left(\operatorname{det} T^{\prime}\right) T^{\prime}$ $\circ S_{\theta_{0}} \circ T^{\prime-1}$, we have $S_{\theta}\left(v_{i}\right)=\left(\operatorname{det} T^{\prime}\right) v_{i}=v_{i}(1 \leq i \leq 3)$ and $S_{\theta}\left(v_{i}\right)=\left(-\operatorname{det} T^{\prime}\right) v_{i}$ $=-v_{i}(4 \leq i \leq 6)$, and hence $\operatorname{det} T^{\prime}=1$ i.e. $T^{\prime} \in S L\left(V_{K}\right)$.

The existence of the equivariant polynomial map $\theta \mapsto S_{\theta}$ of degree 2 from $V_{3}$ to $\mathcal{g}(6, C)$ is based on the fact that $S^{2}\left(\Lambda_{3}\right)=2 \Lambda_{3} \otimes\left(\Lambda_{1}+\Lambda_{5}\right)$ i.e.

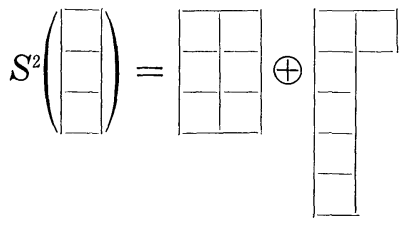

by the Young diagram. The representation $\left(\Lambda_{1}+\Lambda_{5}\right)$ of $G L(6)$ is the composition of the adjoint representation of $S L(6)$ and scalar multiplications (det $g)(g \in G L(6))$. We can also say that the existence of a relative invariant of degree 4 is based on the fact that
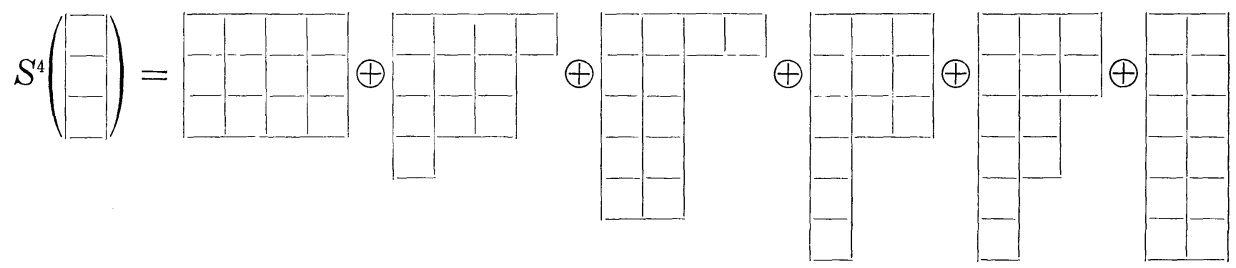

for $G L(6)$. The representation space $2 \Lambda_{6}$ is one-dimensional and the action of $G L(6)$ is given by scalar multiplications $(\operatorname{det} g)^{2}$ for $g \in G L(6)$.

We shall determine the explicit form of $P(x)$ for $x=\sum x_{i j k} u_{i} \wedge u_{j} \wedge$ $u_{k} \in V(20)$ where $\left(x_{i j k}\right)$ forms an alternating tensor of rank three with coefficients in $\boldsymbol{C}$.

For example $x_{123}=x_{231}=x_{312}=-x_{132}=-x_{321}=-x_{213}$. Since deg $P(x)$ $=4, P(x)$ is a linear combination of $x_{i_{1} i_{2} i_{3}} x_{i_{4} i_{5} i_{6}} x_{i_{7} i_{8} i_{9}} x_{i_{10} i_{11} i_{12}}$ where $1 \leq i_{1}$, $\cdots, i_{12} \leq 6$. Under the action of $u_{i} \mapsto a u_{i}, u_{j} \mapsto u_{j}(j \neq i)$ for $1 \leq i \leq 6$, each term $x_{i_{1} i_{2} i_{3}} \cdots x_{i_{10} i_{11} i_{12}}$ is multiplied by $a^{2}$ and hence we have $\left\{i_{1}, \cdots, i_{12}\right\}$ $=\{1,1,2,2, \cdots, 6,6\}$ as a set. Moreover $P(x)$ is invariant under the action of $\widetilde{S}_{6} \subset G L(6)$ where $\widetilde{S}_{6}$ denotes the permutation group of $\{1, \cdots, 6\}$ 
and hence $P(x)$ is of the form

$$
\begin{aligned}
P(x)= & a \sum_{\sigma \in \mathfrak{S}_{6}}^{\prime} x_{\sigma(1) \sigma(2) \sigma(3)}^{2} x_{\sigma(4) \sigma(5) \sigma(6)}^{2}+b \sum_{\sigma \in \mathfrak{G}_{6}}^{\prime} x_{\sigma(1) \sigma(2) \sigma(3)} x_{\sigma(1) \sigma(2) \sigma(4)} x_{\sigma(3) \sigma(5) \sigma(6)} x_{\sigma(4) \sigma(5) \sigma(6)} \\
& +c \sum_{\sigma \in \mathfrak{G}_{6}}^{\prime} x_{\sigma(1) \sigma(2) \sigma(3)} x_{\sigma(1) \sigma(4) \sigma(5)} x_{\sigma(2) \sigma(4) \sigma(6)} x_{\sigma(3) \sigma(5) \sigma(6)}
\end{aligned}
$$

where $\Sigma^{\prime}$ denotes the sum of distinct terms.

Since $P\left(\theta_{0}\right)=1$ for $\theta_{0}=u_{1} \wedge u_{2} \wedge u_{3}+u_{4} \wedge u_{5} \wedge u_{6}$, we have $a=1$. For $\theta=u_{1} \wedge u_{2} \wedge u_{3}+u_{1} \wedge u_{2} \wedge u_{4}+u_{3} \wedge u_{5} \wedge u_{6}+u_{4} \wedge u_{5} \wedge u_{6}$ we have $S_{\theta}\left(u_{i}\right)=0(1 \leq i \leq 6)$ from $\tau \otimes S_{\theta}\left(u_{i}\right)=\left(\left(u_{i} \wedge \theta\right) \otimes 1\right) \wedge D_{3}(\theta)$. Hence we have $P(\theta)=2 a+b=2+b=0$ i.e. $b=-2$. For $\theta=u_{1} \wedge u_{2} \wedge u_{3}+$ $u_{1} \wedge u_{4} \wedge u_{5}+u_{2} \wedge u_{4} \wedge u_{6}+u_{3} \wedge u_{5} \wedge u_{6}$, we have $S_{\theta}\left(u_{1}\right)=-2 u_{6}, S_{\theta}\left(u_{6}\right)$ $=-2 u_{1}$ and hence $P(\theta) u_{1}=S_{\theta}^{2}\left(u_{1}\right)=S_{\theta}\left(-2 u_{6}\right)=4 u_{1}$ i.e. $P(\theta)=c=4$. Hence we have $a=1, b=-2$, and $c=4$.

We can also express $P(x)$ as follows.

Put

$$
X=\left(\begin{array}{lll}
x_{423} & x_{143} & x_{124} \\
x_{523} & x_{153} & x_{125} \\
x_{623} & x_{163} & x_{126}
\end{array}\right) \text { and } X=\left(\begin{array}{lll}
x_{156} & x_{416} & x_{451} \\
x_{256} & x_{426} & x_{452} \\
x_{356} & x_{436} & x_{453}
\end{array}\right) \text {. }
$$

We denote by $X_{i j}$ (resp. $Y_{i j}$ ) the matrix obtained from $X$ (resp. $Y$ ) by crossing out its $i$-th line and $j$-th column. Put $x_{0}=x_{123}$ and $y_{0}=x_{456}$. Then we have

$$
\begin{aligned}
P(x)= & \left(x_{0} y_{0}-\operatorname{tr} X Y\right)^{2}+4 x_{0} \operatorname{det} Y+4 y_{0} \operatorname{det} X \\
& -4 \sum_{i, j} \operatorname{det}\left(X_{i j}\right) \cdot \operatorname{det}\left(Y_{j i}\right) .
\end{aligned}
$$

One can also use Proposition 15 in $\S 4$ to check that the degree of the relative invaiant is four since the orbit of $X_{0}^{\prime}=u_{1} \wedge u_{2} \wedge u_{3}+u_{1} \wedge u_{4} \wedge u_{5}$ $+u_{2} \wedge u_{4} \wedge u_{6}$ is of codimension one.

$$
\left(G L(7), \Lambda_{3}, V(35)\right)
$$

Let $V_{1}$ be a vector space spanned by $u_{1}, \cdots, u_{7}$. Then $G L(7)$ acts on $V_{1}$ as $\rho_{1}(g)\left(u_{1}, \cdots, u_{7}\right)=\left(u_{1}, \cdots, u_{7}\right) g$ for $g \in G L(7)$. Let $V$ be a vector space spanned by skew tensors $u_{i} \wedge u_{j} \wedge u_{k}(1 \leq i<j<k \leq 7)$. Then $G L(7)$ acts on $V$ as $\rho(g)\left(u_{i} \wedge u_{j} \wedge u_{k}\right)=\rho_{1}(g) u_{i} \wedge \rho_{1}(g) u_{j} \wedge \rho_{1}(g) u_{k}$ for $g \in G L(7)$, and $\rho=\Lambda_{3}, V(35)=V$. We shall prove the prehomogeneity by seeking a generic point $X_{0}$ in $V$. Let $H$ be a subgroup of $G L(7)$ defined by $H$ $=\left\{g=\left(g_{i j}\right) \in G L(7) \mid g_{k 1}=0\right.$ for $\left.2 \leq k \leq 7\right\}$. Then $H$ is isomorphic to 
$(G L(1) \times G L(6)) \cdot\left(G_{a}\right)^{6}$ and $G L(6)$ acts prehomogeneously on the subspace of $V$ spanned by $u_{i} \wedge u_{j} \wedge u_{k}(2 \leq i<j<k \leq 7)$. As we have seen in (7), $u_{2} \wedge u_{3} \wedge u_{4}+u_{5} \wedge u_{6} \wedge u_{7}$ is a generic point and a generic isotropy subgroup is isomorphic to $(S L(3) \times S L(3)) \times\{ \pm 1\}$. Therefore we may assume that $X_{0}$ is of the form

$$
u_{2} \wedge u_{3} \wedge u_{4}+u_{5} \wedge u_{6} \wedge u_{7}+u_{1} \wedge\left(\sum_{2 \leq i<j \leq 7} a_{i j} u_{i} \wedge u_{j}\right),
$$

and $(G L(1) \times S L(3) \times S L(3)) \cdot\left(G_{a}\right)^{6}$ leaves this form invariant. By the action of $\left(G_{a}\right)^{6}$, we may assume that $a_{i j}=0(2 \leq i, j \leq 4$ or $5 \leq i, j \leq 6)$. In fact for $g=\left(g_{i j}\right)$ where $g_{i i}=1(1 \leq i \leq 7), g_{12}=-a_{34}, g_{13}=a_{24}, g_{14}$ $=-a_{23}, g_{15}=-a_{67}, g_{16}=a_{57}, g_{17}=-a_{56}$, all remaining entries 0 , we have

$$
\begin{aligned}
\rho(g) X_{0}= & u_{2} \wedge u_{3} \wedge u_{4}+u_{5} \wedge u_{6} \wedge u_{7} \\
& +u_{1} \wedge\left(\sum_{i=2}^{4} \sum_{j=5}^{7} a_{i j} u_{i} \wedge u_{j}\right),
\end{aligned}
$$

and $G L(1) \times S L(3) \times S L(3) \cong S L(3) \times G L(3)$ leaves this form invariant. The action of $S L(3) \times G L(3)$ on the $3 \times 3$ matrices $\left(a_{i j}\right)_{\substack{2 \leq i \leq 4 \\ 5 \leq j \leq 7}}$ is isomorphic to a triplet $\left(S L(3) \times G L(3), \Lambda_{1} \otimes \Lambda_{1}, V(3) \otimes V(3)\right)$. As we have seen in (2), it is a regular trivial P.V. and $\left(a_{i j}\right)=I_{3}$ is a generic point with the isotropy subgroup $S L(3)$. Hence we have $X_{0}=u_{2} \wedge u_{3} \wedge u_{4}+u_{5} \wedge u_{6} \wedge u_{7}$ $+u_{1} \wedge\left(u_{2} \wedge u_{5}+u_{3} \wedge u_{6}+u_{4} \wedge u_{7}\right)$ and the (connected component) of the isotropy subgroup $H_{X_{0}}$ of $H$ is isomorphic to $S L(3)$. Since $\operatorname{dim} H_{X_{0}}=8$ $=\operatorname{dim} H-\operatorname{dim} V(35)$, a triplet $\left(H,\left.\Lambda_{3}\right|_{H}, V_{(35)}\right.$ is a P.V. and hence a triplet $\left(G L(7), \Lambda_{3}, V(35)\right)$ is a P.V. with a generic point $X_{0}=u_{2} \wedge u_{3} \wedge u_{4}+$ $u_{5} \wedge u_{6} \wedge u_{7}+u_{1} \wedge\left(u_{2} \wedge u_{5}+u_{3} \wedge u_{6}+u_{4} \wedge u_{7}\right)$. Let $g_{X_{0}}$ be the isotropy subalgebra of $\mathrm{g}=\mathrm{gl}(7, C)$ at $X_{0}$, and let $\mathfrak{h}$ be the Lie algebra of $H$, i.e., $\mathfrak{h}=\left\{A=\left(a_{i j}\right) \in \operatorname{gr}(7) \mid a_{k 1}=0\right.$ for $\left.2 \leq k \leq 7\right\}$. Then we have $\operatorname{dim} \mathfrak{g}_{x_{0}}=$ $=\operatorname{dim} G L(7)-\operatorname{dim} V(35)=14$ and

$$
\mathfrak{g}_{X_{0}} \cap \mathfrak{h}=\left\{\left(-\frac{X}{}|-| X \in \mathfrak{I l}(3, C)\right\} .\right.
$$

Therefore to determine $\mathrm{g}_{X_{0}}$, it is sufficient to show that $d \rho\left(A_{i}\right) X_{0}=0$ $(1 \leq i \leq 6)$ where $A_{1}$ (resp. $A_{2}, \cdots, A_{6}$ ) is the matrix of the form (1.8) in Example 30 in $\S 1$ with $a=1$ (resp. $b=1, \cdots, f=1$ ), all remaining entries zero.

For example we shall show that $d \rho\left(A_{1}\right) X_{0}=0$. Since $A_{1}$ acts on $V_{1}$ 
as $u_{1} \mapsto u_{2}, u_{3} \mapsto u_{7}, u_{4} \mapsto-u_{6}, u_{5} \mapsto 2 u_{1}, u_{j} \mapsto 0(j=2,6,7)$, we have

$$
\begin{aligned}
& d \rho\left(A_{1}\right) u_{2} \wedge u_{3} \wedge u_{4}=u_{2} \wedge u_{7} \wedge u_{4}-u_{2} \wedge u_{3} \wedge u_{6}, \\
& d \rho\left(A_{1}\right) u_{5} \wedge u_{6} \wedge u_{7}=2 u_{1} \wedge u_{6} \wedge u_{7}, \\
& \quad d \rho\left(A_{1}\right) u_{1} \wedge\left(u_{2} \wedge u_{5}+u_{3} \wedge u_{6}+u_{4} \wedge u_{7}\right) \\
& \quad=u_{2} \wedge\left(u_{3} \wedge u_{6}+u_{4} \wedge u_{7}\right)+u_{1} \wedge\left(u_{7} \wedge u_{6}-u_{6} \wedge u_{7}\right),
\end{aligned}
$$

and hence $d \rho\left(A_{1}\right) X_{0}=0$.

This shows that the isotropy subalgebra $g_{X_{0}}$ at $X_{0}$ is a simple Lie algebra of type $\left(G_{2}\right)$ (See Example 30 in $\S 1$ ) and hence a triplet $(G L(7)$, $\left.\Lambda_{3}, V(35)\right)$ is a regular P.V. by Proposition 25 in $\S 4$.

Now we shall determine the isotropy subgroup $G_{X_{0}}$, not infinitesimally but globally. First we shall show that $G_{X_{0}} \subset G O(7)$. There exists, up to constant, uniquely a quadratic form $q(x)={ }^{t} x A x(x \in V(7), A \in M(7))$ which is invariant under the action of $g_{x_{0}}$ i.e. ${ }^{t} B A+A B=0$ for any $B \in \mathfrak{B}_{x_{0}}$ (See the case of $d=1$ in (32)). In general the quadratic form $q\left(g^{-1} x\right)={ }^{t} x\left({ }^{t} g^{-1} A g^{-1}\right) x$ is invariant under the action of the isotropy subalgebra $\mathfrak{g}_{g X_{0}}=g g_{X_{0}} g^{-1}$ at $g X_{0}$ where $g \in G L(7)$. In particular, if $g \in G_{X_{0}}$ i.e. $g X_{0}=X_{0}, q\left(g^{-1} x\right)$ is invariant under the action of $\mathfrak{g}_{x_{0}}$ and hence it must coincide with $q(x)$ up to a constant multiple. This implies that $G_{x_{0}} \subset G O(7)$. Next we shall show that an element $T$ of $G O(7)$ can be written uniquely as $T=c I_{7} \cdot T_{0}$ where $c \in C^{\times}$and $T \in S O(7)$.

Assume that $T=c T_{0}=c^{\prime} T_{0}^{\prime}$ where $T_{0}, T_{0}^{\prime} \in S O(7)$. Put $a=c^{\prime} c^{-1}$. Then we have $a I_{7}=T_{0} T_{0}^{\prime-1} \in S O(7)$ and hence $\operatorname{det}\left(a I_{7}\right)=a^{7}=1, a^{2} A=A$, i.e., $a^{2}=1$ where $q(x)={ }^{t} x A x$. Therefore we have $a=1$, i.e., $c=c^{\prime}$ and hence $T_{0}=T_{0}^{\prime}$. Finally we shall show that $G_{X_{0}}$ can be written as the direct product of the connected component $G_{X_{0}}^{0}$ and the finite group $H$ which is contained in the centralizer of $G_{X_{0}}^{0}$ in $G L(7)$. Note that by the Schur's lemma, an element of $H$ is of the form $c I_{7}$ where $c \in C^{\times}$.

In general let $G$ be a connected and simply connected semi-simple algebraic group with the Lie algebra $g$. Then we have the exact sequence $1 \rightarrow Z(G) \rightarrow G \rightarrow$ Aut $(\mathfrak{g}) \rightarrow$ Aut (Dynkin) $\rightarrow 1$ where $Z(G)$ is the center of $G$ and Aut (Dynkin) is the automorphism of the Dynkin diagram of $g$. Since the Dynkin diagram of $g_{x_{0}}=\left(g_{2}\right)$ is $0 \Longrightarrow 0$, we have Aut (Dynkin) $=1$ and hence $G \rightarrow$ Aut $\left(g_{x_{0}}\right) \rightarrow 1$ (exact). Since $G$ is connected, Aut $\left(g_{x_{0}}\right)$ is also connected. On the other hand, we have the exact sequence

$$
1 \longrightarrow H \longrightarrow G_{X_{0}} \stackrel{\varphi}{\longrightarrow} \operatorname{Aut}\left(g_{X_{0}}\right) \longrightarrow 1
$$


where $\varphi$ is defined by $\varphi(g) X=g X g^{-1}$ for $g \in G_{X_{0}}$ and $X \in \mathfrak{g}_{X_{0}}$. As we have $g \mathfrak{g}_{X_{0}} g^{-1}=\mathfrak{g}_{\rho(g) X_{0}}=\mathfrak{g}_{X_{0}}$ for $g \in G_{X_{0}}, \varphi(g)$ is well-defined for $g \in G_{X_{0}}$. Since the Lie algebra of $G_{X_{0}}^{0}$ and that of Aut $\left(\mathfrak{g}_{X_{0}}\right)$ are $\mathfrak{g}_{X_{0}}, \rho\left(G_{X_{0}}^{0}\right)$ is an open subgroup (and hence closed subgroup) of Aut $\left(\mathfrak{g}_{X_{0}}\right)$, and hence we have $\rho\left(G_{X_{0}}^{0}\right)=\operatorname{Aut}\left(\mathfrak{g}_{X_{0}}\right)$. This implies that an element $T$ of $G_{X_{0}}$ can be written as $T=c I \cdot T_{0}$ where $c I \in H$ and $T_{0} \in G_{X_{0}}^{0} \subset S O(7)$. Since this expression is unique, we have $G_{X_{0}}=G_{X_{0}}^{0} \times H$.

If $c I_{7} \in H$, then $\rho\left(c I_{7}\right) X_{0}=c^{3} X_{0}=X_{0}$, and hence we have $G_{X_{0}}=\left(G_{2}\right) \times$ $\left\{\omega I_{7} \mid \omega^{3}=1\right\}$. Any rational character $\chi$ of $G L(7)$ can be written as $\chi(g)$ $=(\operatorname{det} g)^{m}$ for some integer $m(g \in G L(7))$. Since $\left.\chi\right|_{G_{x_{0}}}=1$ if and only if $m \equiv 0 \bmod 3$, by Proposition 19 in $\S 4$, any relative invariant is of the form $c f(x)^{\ell}\left(c \in C^{\times}, \ell \in Z\right)$ where $f(x)$ is the relative invariant with the character $(\operatorname{det} g)^{3}$. For $g=t I_{7} \in G L(7)$, we have $(\operatorname{det} g)^{3}=t^{21}=t^{3 \operatorname{deg} f(x)}$, i.e., $\operatorname{deg} f(x)=7$.

Proposition 8. A triplet $\left(G L(7), \Lambda_{3}, V(35)\right)$ is a regular P.V. and its generic isotropy subgroup is $\left(G_{2}\right) \times\left\{\omega I_{7} \mid \omega^{3}=1\right\}$. The relative invariant is of degree 7 .

Remark 9. Let $W$ be the totality of $7 \times 7$ symmetric matrices. By the inner product $\langle X, Y\rangle=\operatorname{tr} X Y(X, Y \in W)$, we may identify the dual vector space $W^{*}$ of $W$ with $W$. Since the symmetric product $S^{3}(-$

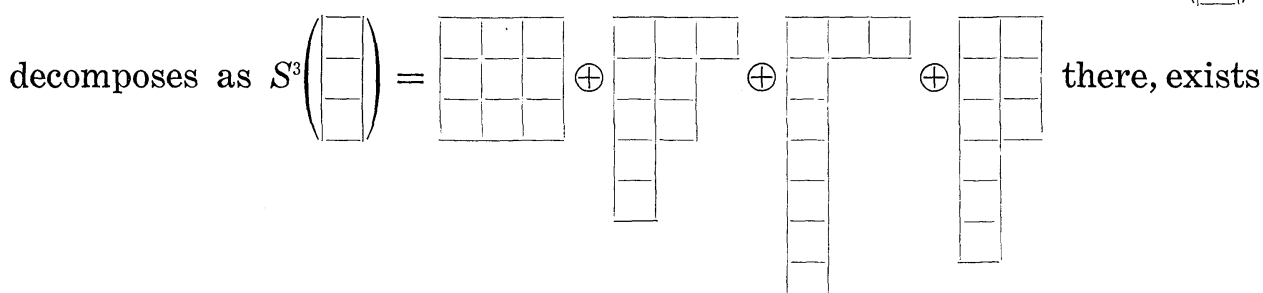
a polynomial map $\varphi$ of degree 3 from $V(35)$ to $W$ satisfying $\varphi(\rho(g) x)=$ $(\operatorname{det} g) g \cdot \varphi(x)^{t} g$ for $g \in G L(7), x \in V(35)$. On the other hand, $S^{4}(-)$ contains - Note that this fact corresponds to the existence of the 
relative invariant of degree 4 of $\left(G L(6), \Lambda_{3}, V(20)\right)$. Therefore there exists a polynomial map $\varphi^{*}$ of degree 4 from $V(35)$ to $W^{*}$ satisfying $\varphi^{*}(\rho(g) x)$ $=(\operatorname{det} g)^{2} \cdot{ }^{t} g^{-1} \varphi^{*}(x) g^{-1}$. Hence $f(x)=\left\langle\varphi(x), \varphi^{*}(x)\right\rangle=\operatorname{tr} \varphi(x) \cdot \varphi^{*}(x)$ is a relative invariant of degree 7 satisfying $f\left((\rho(g) x)=(\operatorname{det} g)^{3} f(x)\right.$ for $g \in G L(7)$ and $x \in V(35)$. However, it is necessary to prove that $\left\langle\varphi(x), \varphi^{*}(x)\right\rangle$ is not identically zero.

One can also use Proposition 15 in $\S 4$ to check that $\operatorname{deg} f=7$ since the orbit of $X_{0}^{\prime}=u_{2} \wedge u_{3} \wedge u_{5}+u_{3} \wedge u_{4} \wedge u_{6}+u_{1} \wedge u_{2} \wedge u_{7}-u_{1} \wedge u_{4} \wedge u_{5}$ is of codimension one.

$$
\left(G L(8), \Lambda_{3}, V(56)\right)
$$

Let $V_{1}$ be a vector space spanned by $u_{1}, \cdots, u_{8}$ over $C$. Then $G L(8)$ acts on $V_{1}$ as $\rho_{1}(g)\left(u_{1}, \cdots, u_{8}\right)=\left(u_{1}, \cdots, u_{8}\right) g$ for $g \in G L(8)$. Let $V$ be a vector space spanned by skew-tensors $u_{i} \wedge u_{j} \wedge u_{k}(1 \leq i<j<k \leq 8)$ of rank 3 over $C$. We identify $V(56)$ with $V$. Then the action $\rho=\Lambda_{3}$ of $G L(8)$ is given by $\rho(g)\left(u_{i} \wedge u_{j} \wedge u_{k}\right)=\rho_{1}(g) u_{i} \wedge \rho_{1}(g) u_{j} \wedge \rho_{1}(g) u_{k}(1 \leq i<j$ $<k \leq 8$ ). We shall determine the prehomogeneity by seeking a generic point $X_{0}$. By the action of $G L(7)$, we have $X_{0}=\omega_{0}+u_{8} \wedge \eta$ where $\omega_{0}=$ $u_{2} \wedge u_{3} \wedge u_{4}+u_{5} \wedge u_{6} \wedge u_{7}+u_{1} \wedge\left(u_{2} \wedge u_{5}+u_{3} \wedge u_{6}+u_{4} \wedge u_{7}\right)$ and $\eta$ is a 2 -form of $u_{1}, \cdots, u_{7}$ (see (8)). The isotropy subgroup $\left(G_{2}\right)$ of $G L(7)$ at $\omega_{0}$ acts on the space $V(21)$ of all 2 -forms of $u_{1}, \cdots, u_{7}$. Let $\mathfrak{h}$ be the Cartan subalgebra of $\left(g_{2}\right)$ defined in Example 30 in $\S 1$. Then the weights of this action of $\left(\mathfrak{g}_{2}\right)$ on $V(21)$ w.r.t. $\mathfrak{h}$ is given by $\left\{0, \pm \lambda_{1}, \pm \lambda_{2}, \pm\left(\lambda_{1}+\lambda_{2}\right)\right\}$ $\cup\left\{0,0, \pm \lambda_{1}, \pm \lambda_{2}, \pm \lambda_{1} \pm \lambda_{2}, \pm\left(\lambda_{1}+2 \lambda_{2}\right), \pm\left(2 \lambda_{1}+\lambda_{2}\right)\right\}$ and hence this action of $\left(G_{2}\right)$ is $\Lambda_{1} \oplus \Lambda_{2}$, i.e., $V(21)$ decomposes into the direct sum of the 7-dimensional representation space $V(7)$ and the adjoint representation space $V(14)$ $\cong\left(\mathfrak{g}_{2}\right): V(21)=V(7) \oplus V(14)$ (see Definition 4 and Example 30 in $\S 1$ ).

Let $\eta_{i}=\partial \omega_{0} / \partial u_{i}(1 \leqq i \leqq 7)$ be the polarizations of $\omega_{0}$ i.e. $\eta_{1}=u_{2} \wedge$ $u_{5}+u_{3} \wedge u_{6}+u_{4} \wedge u_{7}, \eta_{2}=u_{3} \wedge u_{4}-u_{1} \wedge u_{5}, \eta_{3}=-u_{2} \wedge u_{4}-u_{1} \wedge u_{6}, \eta_{4}$ $=u_{2} \wedge u_{3}-u_{1} \wedge u_{7}, \eta_{5}=u_{6} \wedge u_{7}+u_{1} \wedge u_{2}, \eta_{6}=-u_{5} \wedge u_{7}+u_{1} \wedge u_{3}, \eta_{7}=$ $u_{5} \wedge u_{6}+u_{1} \wedge u_{4}$.

Since

$$
\begin{aligned}
\frac{\partial \omega_{0}}{\partial u_{i}}\left(u_{1}, \cdots, u_{7}\right) & =\frac{\partial}{\partial u_{i}} \omega_{0}\left(\left(u_{1}, \cdots, u_{7}\right) g\right) \\
& =\sum_{k} \frac{\partial \omega_{0}}{\partial u_{k}}\left(\left(u_{1}, \cdots, u_{7}\right) g\right) \frac{\partial \sum_{j} u_{j} g_{j k}}{\partial u_{i}}
\end{aligned}
$$




$$
=\sum_{k} \frac{\partial \omega_{0}}{\partial u_{k}}\left(\left(u_{1}, \cdots, u_{7}\right) g\right) g_{i k}
$$

for $g=\left(g_{i j}\right) \in\left(G_{2}\right)$, we have

$$
\left(\frac{\partial \omega_{0}}{\partial u_{1}}\left(\left(u_{1}, \cdots, u_{7}\right) g\right), \cdots, \frac{\partial \omega_{0}}{\partial u_{7}}\left(\left(u_{1}, \cdots, u_{7}\right) g\right)=\left(\frac{\partial \omega_{0}}{\partial u_{1}}, \cdots, \frac{\partial \omega_{0}}{\partial u_{7}}\right)^{t} g^{-1}\right.
$$

for $g \in\left(G_{2}\right)$, and hence $\left\{\eta_{1}, \cdots, \eta_{7}\right\}$ is a basis of $V(7)$. Put $X_{0}=\omega_{0}+u_{8} \wedge \eta^{\prime}$ $+u_{8} \wedge \eta_{0}$ where $\eta_{0}=a_{1} \eta_{1}+\cdots+a_{7} \eta_{7}$ and $\eta^{\prime} \in V(14)$. Then by the action of $u_{\nu} \mapsto u_{\nu}-a_{\nu} u_{8}(1 \leq \nu \leq 7)$ and $u_{8} \mapsto u_{8}$, we have $X_{0}=\omega_{0}+u_{8} \wedge \eta^{\prime}$ where $\eta^{\prime} \in V(14) \cong\left(\mathfrak{g}_{2}\right)$. Moreover we may assume that $\eta^{\prime}$ is a regular element of $\left(g_{2}\right)$ (see (3)). A regular element $\eta^{\prime}$ can be transferred to an element of the Cartan subalgebra $\mathfrak{h}$ by the action of $\left(G_{2}\right)$. We shall determine the subspace of $V(14)$ corresponding to $\mathfrak{h}$. Let $\varphi:\left(\mathfrak{g}_{2}\right) \rightarrow V(14)$ be the $\left(G_{2}\right)$-equivariant isomorphism. Since $\mathfrak{h}$ is abelian, we have $A \varphi(X)=$ $\varphi([A, X])=\varphi(0)=0$ for $A, X \in \mathfrak{h}$, and hence $\varphi(\mathfrak{h})=\{x \in V(14) \mid A x=0$ for any $A \in \mathfrak{h}\}=\left\{c_{1} u_{2} \wedge u_{5}+c_{2} u_{3} \wedge u_{6}+c_{3} u_{4} \wedge u_{7} \mid c_{1}+c_{2}+c_{3}=0\right\}$.

Therefore we may assume that $X_{0}=\omega_{0}+u_{8} \wedge\left(c_{1} u_{2} \wedge u_{5}+c_{2} u_{3} \wedge u_{6}\right.$ $+c_{3} u_{4} \wedge u_{7}$ ) with $c_{1}+c_{2}+c_{3}=0$. By changing indices and generalizing this form, we shall consider the 6-dimensional subvariety $V^{\prime}$ of $V$ consisting of the forms $u_{1} \wedge u_{2} \wedge u_{3}+u_{4} \wedge u_{5} \wedge u_{6}+u_{7} \wedge\left(a_{1} u_{1} \wedge u_{4}+a_{2} u_{2} \wedge\right.$ $\left.u_{5}+a_{3} u_{3} \wedge u_{6}\right)+u_{8} \wedge\left(b_{1} u_{1} \wedge u_{4}+b_{2} u_{2} \wedge u_{5}+b_{3} u_{3} \wedge u_{6}\right)$. Let $H$ be the subgroup of $G L(8)$ defined as follows

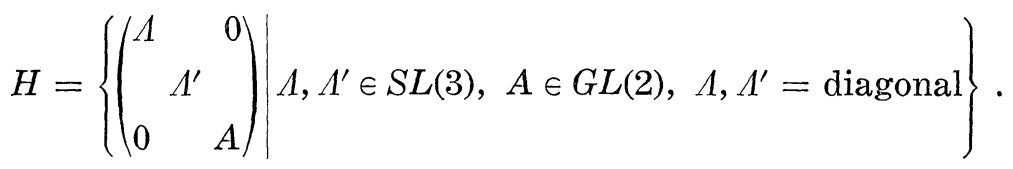

Then the subgroup $H$ acts on the subvariety $V^{\prime}$ as

$$
X=\left(\begin{array}{lll}
a_{1} & a_{2} & a_{3} \\
b_{1} & b_{2} & b_{3}
\end{array}\right) \mapsto \Lambda \Lambda^{\prime} X A
$$

Obviously this action is prehomogeneous, and we may take $X=$ ${ }^{t}\left(\begin{array}{rrr}1 & -1 & 0 \\ 1 & 0 & -1\end{array}\right)$ as a generic point. Hence the triplet $\left(G L(8), \Lambda_{3}, V(56)\right)$ is a $\mathrm{P}$. V. and the corresponding point $X_{0}=u_{1} \wedge u_{2} \wedge u_{3}+u_{4} \wedge u_{5} \wedge u_{6}$ $+u_{7} \wedge\left(u_{1} \wedge u_{4}-u_{2} \wedge u_{5}\right)+u_{8} \wedge\left(u_{1} \wedge u_{4}-u_{3} \wedge u_{6}\right)$ is a generic point. We shall calculate the isotropy subalgebra $g_{X_{0}}$ at $X_{0}$. The infinitesimal action $d \rho$ of $\rho$ is given by $d \rho(A)\left(u_{i} \wedge u_{j} \wedge u_{k}\right)=d \rho_{1}(A) u_{i} \wedge u_{j} \wedge u_{k}+u_{i}$ 
$\wedge d \rho_{1}(A) u_{j} \wedge u_{k}+u_{i} \wedge u_{j} \wedge d \rho_{1}(A) u_{k}$. For example $d \rho(A)\left(u_{1} \wedge u_{2} \wedge u_{3}\right)=$ $\left(a_{11} u_{1}+a_{41} u_{4}+a_{51} u_{5}+a_{61} u_{6}+a_{71} u_{7}+a_{81} u_{8}\right) \wedge u_{2} \wedge u_{3}+u_{1} \wedge\left(a_{22} u_{2}+a_{42} u_{4}+\right.$ $\left.a_{52} u_{5}+a_{62} u_{6}+a_{72} u_{7}+a_{82} u_{8}\right) \wedge u_{3}+u_{1} \wedge u_{2} \wedge\left(a_{33} u_{3}+a_{43} u_{4}+a_{53} u_{5}+a_{63} u_{6}+\right.$ $\left.a_{73} u_{7}+a_{83} u_{8}\right)$ where $A=\left(a_{i j}\right) \in \mathfrak{g} \mathfrak{l}(8)$. Such a calculation shows that $d \rho(A) X_{0}=\sum a_{i j k} u_{i} \wedge u_{j} \wedge u_{k}$ where $a_{i j k}$ are given by the following table.

\begin{tabular}{c|c||c|c||c|c||c|c}
\hline$i j k$ & $a_{i j k}$ & $i j k$ & $a_{i j k}$ & $i j k$ & $a_{i j k}$ & $i j k$ & $a_{i j k}$ \\
\hline 123 & $a_{11}+a_{22}+a_{33}$ & 148 & $a_{11}+a_{44}+a_{88}+a_{87}$ & 247 & $a_{21}-a_{45}$ & 358 & $-a_{56}$ \\
124 & $a_{43}-a_{27}-a_{28}$ & 156 & $a_{14}$ & 248 & $a_{21}$ & 367 & $-a_{78}$ \\
125 & $a_{53}-a_{17}$ & 157 & $a_{54}-a_{12}$ & 256 & $a_{24}-a_{67}$ & 368 & $-a_{33}-a_{66}-a_{88}$ \\
126 & $a_{63}$ & 158 & $a_{54}$ & 257 & $-a_{22}-a_{55}-a_{77}$ & 378 & $-a_{76}$ \\
127 & $a_{15}+a_{24}+a_{73}$ & 167 & $a_{64}$ & 258 & $-a_{87}$ & 456 & $a_{44}+a_{55}+a_{66}$ \\
128 & $a_{24}+a_{83}$ & 168 & $a_{64}-a_{13}$ & 267 & $-a_{65}$ & 457 & $a_{76}-a_{51}-a_{42}$ \\
134 & $-a_{37}-a_{38}-a_{42}$ & 178 & $a_{74}-a_{84}$ & 268 & $-a_{23}$ & 458 & $a_{86}-a_{51}$ \\
135 & $-a_{52}$ & 234 & $a_{41}$ & 278 & $a_{85}$ & 467 & $-a_{61}-a_{75}$ \\
136 & $-a_{18}-a_{62}$ & 235 & $a_{37}+a_{51}$ & 345 & $a_{36}$ & 468 & $-a_{43}-a_{61}-a_{85}$ \\
137 & $a_{34}-a_{72}$ & 236 & $a_{61}-a_{28}$ & 346 & $a_{48}-a_{35}$ & 478 & $a_{81}-a_{71}$ \\
138 & $a_{16}+a_{34}-a_{82}$ & 237 & $a_{71}-a_{35}$ & 347 & $a_{31}$ & 567 & $a_{62}+a_{74}$ \\
145 & $a_{16}+a_{57}+a_{58}$ & 238 & $a_{26}+a_{81}$ & 348 & $a_{31}-a_{46}$ & 568 & $a_{84}-a_{53}$ \\
146 & $a_{67}+a_{68}-a_{15}$ & 245 & $a_{26}+a_{47}$ & 356 & $a_{34}+a_{58}$ & 578 & $-a_{82}$ \\
147 & $a_{11}+a_{44}+a_{77}+a_{78}$ & 246 & $-a_{25}$ & 357 & $-a_{32}$ & 678 & $a_{73}$ \\
\hline
\end{tabular}

Hence by simple calculation we have $d \rho(A) X_{0}=0$, i.e. $a_{i j k}=0$ for $1 \leq i<j<k \leq 8$, if and only if $A$ is of the following form (5.7).

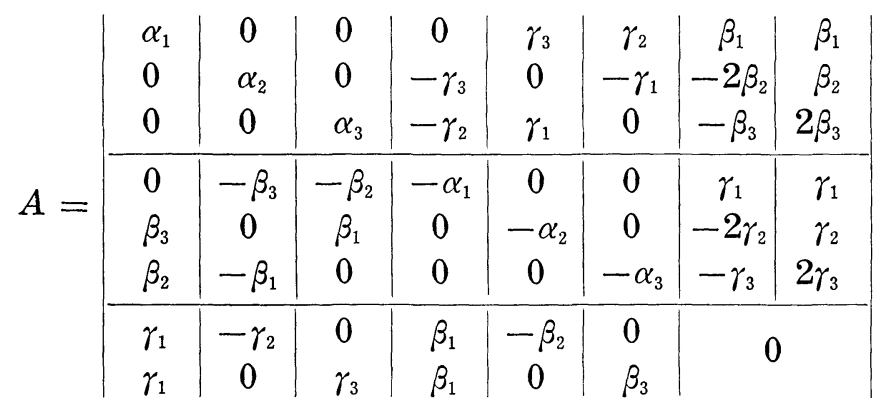

$$
\text { with } \alpha_{1}+\alpha_{2}+\alpha_{3}=0 \text {. }
$$

Let $\mathfrak{h}$ be the subalgebra of $\mathfrak{g}_{X_{0}}=\left\{A \in \mathfrak{g r}(7) \mid d \rho(A) X_{0}=0\right\}$ consisting of the diagonal matrices $H\left(\alpha_{1}, \alpha_{2}, \alpha_{3}\right)$ in (5.7).

For $1 \leq i \leq 3$, let $E_{i}$ (resp. $F_{i}$ ) be the element of the form (5.7) with $\beta_{i}=1$ (resp. $\gamma_{i}=1$ ), all remaining entries zero. Then one can easily check that for each $i=1,2,3$, ad $(H) E_{i}=\alpha_{i} E_{i}$ and $\operatorname{ad}(H) F_{i}=$ 
$-\alpha_{i} F_{i}$ where $H=H\left(\alpha_{1}, \alpha_{2}, \alpha_{3}\right) \in \mathfrak{h}$. This shows that $\mathfrak{h}$ is a Cartan subalgebra of $\mathfrak{g}_{x_{0}}$ and the root system of $\mathfrak{g}_{x_{0}}$ w. r.t. $\mathfrak{h}$ is given by $\Delta=$ $\left\{ \pm \alpha_{1}, \pm \alpha_{2}, \pm \alpha_{3} \mid \alpha_{1}+\alpha_{2}+\alpha_{3}=0\right\}$ i.e. $\mathfrak{g}_{X_{0}} \cong \mathfrak{I l}(3)$ and $A$ in (5.7) is the adjoint representation $\Lambda_{1}+\Lambda_{2}$ of $\mathfrak{g}(3)$. Hence this triplet is regular by Proposition 25 in $\S 4$.

Put $X_{0}^{\prime}=u_{1} \wedge u_{2} \wedge u_{3}+u_{1} \wedge u_{5} \wedge u_{6}+u_{2} \wedge u_{4} \wedge u_{6}+u_{7} \wedge\left(u_{1} \wedge u_{4}-\right.$ $\left.u_{2} \wedge u_{5}\right)+u_{8} \wedge\left(u_{1} \wedge u_{4}-u_{3} \wedge u_{6}\right)$. Then by the same calculation as the case of $X_{0}$, the isotropy subalgebra $\mathfrak{g}_{X_{0}^{\prime}}$ is given by

$$
\begin{aligned}
& \left.\mathfrak{g}_{X_{0}^{\prime}}=\left\{\begin{array}{cc|ccc|ccc}
\alpha & \beta_{1} & \gamma_{2} & \gamma_{3} & \gamma_{4} & -\beta_{3} & 2 \beta_{2} & 2 \beta_{2} \\
\beta_{1} & \alpha & \gamma_{1} & -\gamma_{4} & \gamma_{5} & -\beta_{2} & -4 \beta_{3} & 2 \beta_{3} \\
\hline & & -2 \alpha & \beta_{3} & \beta_{2} & & & \\
0 & -\beta_{3} & -2 \alpha & \beta_{1} & & 0 & \\
& & \beta_{2} & \beta_{1} & -2 \alpha & & & \\
\hline \beta_{3}-\beta_{2} & \gamma_{3}-\gamma_{5} & \gamma_{2} & \gamma_{1} & \alpha & 2 \beta_{1} & -4 \beta_{1} \\
\beta_{2} & \beta_{3} & 0 & -\gamma_{1} & \gamma_{2} & 0 & \alpha & 0 \\
\beta_{2} & 0 & \gamma_{4} & -\gamma_{1} & 0 & -\beta_{1} & 0 & \alpha
\end{array}\right\}\right\} \\
& \left.\cong(\alpha) \oplus\left[\begin{array}{cc}
\frac{\beta_{1}}{2} & \frac{\beta_{2}-\beta_{3}}{2} \\
\frac{\beta_{2}+\beta_{3}}{2} & -\frac{\beta_{1}}{2}
\end{array}\right]\right) \oplus\left(\gamma_{1}, \gamma_{2}, \gamma_{3}, \gamma_{4}, \gamma_{5}\right) \\
& \cong(\mathfrak{g l}(1) \oplus \mathfrak{g l}(2)) \oplus V(5)
\end{aligned}
$$

where the second $\oplus$ denotes the semi-direct sum and $V(5)$ denotes the Lie algebra of the 5-dimensional vector group. Hence the isotropy subgroup $G_{X_{0}^{\prime}}$ at $X_{0}^{\prime}$ is locally isomorphic to $(G L(1) \times S L(2)) \cdot\left(G_{a}\right)^{5}$.

Since $\operatorname{dim} G L(8)-\operatorname{dim} G_{X_{0}^{\prime}}=55$, the orbit of $X_{0}^{\prime}$ is of codimension one. For an element $A$ of $\mathrm{g}_{X_{0}^{\prime}}$, we have $\operatorname{tr}_{V} A=-21 \alpha$ and $\operatorname{tr} \operatorname{ad} g_{X_{0}^{\prime}} A=$ $15 \alpha$, and hence $\operatorname{deg} f=\frac{-21 \alpha+15 \alpha}{-21 \alpha} \times 56=16$ by Proposition 15 in $\S 4$.

The orbital decomposition of this space was completed by I. Ozeki (see [20]).

Proposition 10. A triplet $\left(G L(8), \Lambda_{3}, V(56)\right)$ is a regular P.V. and its generic isotropy subgroup is locally isomorphic to $S L(3)$. The relative invariant is of degree 16.

$$
\left(S L(3) \times G L(2), 2 \Lambda_{1} \otimes \Lambda_{1}, V(6) \otimes V(2)\right) .
$$

We identify $V(6) \otimes V(2)$ with $V=\left\{X=\left(X_{1}, X_{2}\right) \mid X_{1}, X_{2} \in M(3),{ }^{t} X_{1}=\right.$. 
$\left.X_{1},{ }^{t} X_{2}=X_{2}\right\}$. Then the action $\rho=2 \Lambda_{1} \otimes \Lambda_{1}$ of $S L(3) \times G L(2)$ is given by $\rho(A, B) X=\left(A\left(a X_{1}+b X_{2}\right)^{t} A, A\left(c X_{1}+d X_{2}\right)^{t} A\right)$ where $X=\left(X_{1}, X_{2}\right) \in V$, $(A, B) \in S L(3) \times G L(2)$, and $B=\left(\begin{array}{ll}a & b \\ c & d\end{array}\right)$. For each $X=\left(X_{1}, X_{2}\right)$ in $V$, we can obtain the binary cubic form $F_{X}(u, v)=\operatorname{det}\left(u X_{1}+v X_{2}\right)$ which is invariant under the action of $S L(3)$. Let $G_{X_{0}}$ be the isotropy subgroup of $G=S L(3) \times G L(2)$ at $X_{0}=\left(\left(\begin{array}{lll}1 & & \\ & 1 & 1\end{array}\right),\left(\begin{array}{lll}1 & & \\ & & \\ & & \omega^{2}\end{array}\right)\right)$ where $\omega^{3}=1, \omega \neq 1$, and let $(A, B)$ be an element of $G_{X_{0}}$ where $A \in S L(3), B \in G L(2)$. Then $B$ must be in the isotropy subgroup

$$
\left\{\left(\begin{array}{ll}
a & 0 \\
0 & d
\end{array}\right) \mid a^{3}=d^{3}=1\right\} \cup\left\{\left(\begin{array}{ll}
0 & b \\
c & 0
\end{array}\right) \mid b^{3}=c^{3}=1\right\}
$$

of $G L(2)$ at $F_{X_{0}}(u, v)=u^{3}+v^{3}$ (see (6)). In the case of $B=\left(\begin{array}{ll}1 & \\ & 1\end{array}\right)$, we have $A^{t} A=I_{3}, A\left(\begin{array}{ccc}1 & & \\ & \omega & \omega^{2}\end{array}\right)^{t} A=\left(\begin{array}{ccc}1 & & \\ & \omega & \omega^{2}\end{array}\right)$ and hence

$$
A\left(\begin{array}{lll}
1 & & \\
& \omega & \\
& & \omega^{2}
\end{array}\right)=\left(\begin{array}{lll}
1 & & \\
& \omega & \\
& & \omega^{2}
\end{array}\right) A
$$

This implies that $A$ is diagonal. Since $A^{t} A=I_{3}$, we have $A=$ $( \pm 1 \pm 1 \pm 1)$, i.e., $\{A\}$ is an abelian group of order 8 of type $(2,2,2)$. Similarly we have

$$
A=\left(\begin{array}{lll} 
\pm \omega & & \\
& \pm \omega & \\
& & \pm \omega
\end{array}\right)\left(\operatorname{resp.}\left(\begin{array}{lll} 
\pm \omega & & \pm \omega \\
& \pm \omega
\end{array}\right),\left(\begin{array}{lll} 
\pm 1 & & \\
& \pm \omega & \pm \omega^{2}
\end{array}\right)\right)
$$

when $B=\left(\begin{array}{cc}\omega & \\ & \omega\end{array}\right)\left(\operatorname{resp} .\left(\begin{array}{cc}\omega & \\ & \omega^{2}\end{array}\right),\left(\begin{array}{l}1 \\ 1\end{array}\right)\right)$. This implies that $G_{X_{0}}$ is a finite group of order $8 \times 18=144$. Hence it is a regular P.V. by Proposition

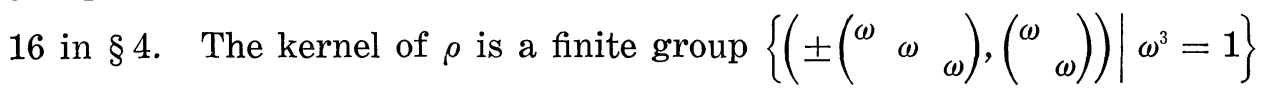
of order 6, and hence the image $\rho\left(G_{X_{0}}\right)$ is a finite group of order 24 . Since $X \mapsto F_{X}(u, v)$ is equivariant, the discriminant $f(X)$ of $F_{X}(u, v)$ is a relative invariant of $S L(3) \times G L(2)$ (see (6)). Any rational character $\chi$ of $S L(3) \times G L(2)$ is of the form $\chi((A, B))=(\operatorname{det} B)^{m}$ for some $m \in Z$ where $(A, B) \in S L(3) \times G L(2)$. Since $\operatorname{deg} f(X)=12$, if $(\operatorname{det} B)^{m}$ is the character of $f(X)$, we have $(\operatorname{det} B)^{m}=t^{2 m}=t^{12}$ for $B=\left(\begin{array}{l}t \\ t\end{array}\right)$ and hence 
$m=6$. Since $\chi \mid G_{X_{0}}=1$ if and only if $m=6 n$ for some $n \in Z$, by Proposition 19 in $\S 4$, any relative invariant is of the form $c f(X)^{n}$ and hence $f(X)$ is irreducible.

Proposition 11. A triplet $\left(S L(3) \times G L(2), 2 \Lambda_{1} \otimes \Lambda_{1}, V(6) \otimes V(2)\right)$ is a regular P.V. and its generic isotropy subgroup is a finite group of order 144. The relative invariant is of degree 12.

$$
\left(S L(n) \times G L(2), \Lambda_{2} \otimes \Lambda_{1}, V\left(\frac{n(n-1)}{2}\right) \otimes V(2)\right) \quad(n \geqq 5)
$$

I) The case of $n=2 m$.

We identify $V=V(m(2 m-1)) \otimes V(2)$ with $\left\{X=\left(X_{1}, X_{2}\right) \in M(2 m) \oplus\right.$ $\left.M(2 m) \mid{ }^{t} X_{1}=-X_{1},{ }^{t} X_{2}=-X_{2}\right\}$. Then the action $\rho=\Lambda_{2} \otimes \Lambda_{1}$ on $V$ is given by $\rho(g) X=\left(A\left(\alpha X_{1}+\beta X_{2}\right)^{t} A, A\left(\gamma X_{1}+\delta X_{2}\right)^{t} A\right)$ for

$$
g=\left(A,\left(\begin{array}{ll}
\alpha & \beta \\
\gamma & \delta
\end{array}\right)\right) \in S L(m) \times G L(2) .
$$

For each $X=\left(X_{1}, X_{2}\right)$ in $V$, we can obtain the binary $m$-form $F_{X}(u, v)=$ $P f f\left(u X_{1}+v X_{2}\right)$ which is invariant under the action of $S L(m)$ where Pff denotes the Pfaffian. This map $\varphi: X \rightarrow F_{X}(u, v)$ is clearly equivariant. Moreover it is generically surjective since $\varphi(X)= \pm\left(u-\lambda_{1} v\right) \cdots\left(u-\lambda_{m} v\right)$ for

$$
X=\left(\left(\begin{array}{c|c}
0 & I_{m} \\
\hline-I_{m} & 0
\end{array}\right),\left(\frac{0}{\Lambda} \mid \begin{array}{l}
-\Lambda \\
\hline 0
\end{array}\right)\right) \quad \text { where } \Lambda=\left(\begin{array}{ccc}
\lambda_{1} & & 0 \\
& \ddots & \\
0 & & \lambda_{m}
\end{array}\right) .
$$

For $m \geqq 4$, a triplet $\left(G L(2), m \Lambda_{1}, V(m+1)\right)$ is not a P.V. and hence the triplet $\left(S L(2 m) \times G L(2), \Lambda_{2} \otimes \Lambda_{1}, V\left(2 m^{2}-m\right) \otimes V(2)\right)$ is not a P.V. for $m \geqq 4$ by Lemma 5 in $\S 2$. Assume that $m=3$. Put

$$
X_{0}=\left\{\left(\begin{array}{c|c}
0 & I_{3} \\
\hline-I_{3} & 0
\end{array}\right),\left(\begin{array}{c|c}
0 & \Lambda \\
\hline-\Lambda & 0
\end{array}\right)\right\} \quad \text { where } \Lambda=\left(\begin{array}{ccc}
1 & & \\
& 0 & \\
& & -1
\end{array}\right) \text {. }
$$

In general the infinitesimal action $d \rho$ of $\rho$ is given by $d \rho\left(A,\left(\begin{array}{ll}a & b \\ c & d\end{array}\right)\right) X=\left(A X_{1}+X_{1}^{t} A+a X_{1}+b X_{2}, A X_{2}+X_{2}^{t} A+c X_{1}+d X_{2}\right)$ where $X=\left(X_{1}, X_{2}\right)$ in $V, A \in \mathfrak{g l}(2 m, C)$ and $\left(\begin{array}{ll}a & b \\ c & d\end{array}\right) \in \mathfrak{g l}(2, C)$. Hence we have $d \rho\left(A,\left(\begin{array}{ll}a & b \\ c & d\end{array}\right)\right) X_{0}=\left(X_{0}^{1}, X_{0}^{2}\right)$ where $A=\left(a_{i j}\right) \in \mathfrak{g l}(2 m)$, and $X_{0}^{1}=\left(c_{i j}\right)$, 
$X_{0}^{2}=\left(c_{i j}^{\prime}\right)$ are $6 \times 6$ skew-symmetric matrices given as follows.

$$
\begin{array}{|c|c||c|c|c|c|}
c_{12} & a_{24}-a_{15} & c_{35} & a_{32}+a_{56} & c_{23}^{\prime} & a_{26} \\
c_{13} & a_{34}-a_{16} & c_{36} & a-a_{33}+a_{66} & a_{21}^{\prime} \\
c_{24} & a+b+a_{11}+a_{44} & c_{45} & a_{42}-a_{51} & c_{25} & c \\
c_{15}^{\prime} & a_{12}+a_{54} & c_{46} & a_{43}-a_{61} & c_{26}^{\prime} & -a_{23} \\
c_{15} & a_{13}+a_{64} & c_{56} & a_{53}-a_{62} & c_{34}^{\prime} & a_{31}-a_{46} \\
c_{16} & a_{35}-a_{26} & c_{12}^{\prime} & a_{24} & -a_{56} \\
c_{23}^{\prime} & c_{35}^{\prime} & a_{16}+a_{34} & c_{36}^{\prime} & c-d-a_{33}-a_{66} \\
c_{24} & a_{21}+a_{45} & c_{13}^{\prime} & c_{14} \\
c_{25} & a+a_{22}+a_{55} & c_{14}^{\prime} & c+d+a_{11}+a_{44} & c_{45}^{\prime} & -a_{51} \\
c_{26} & a_{23}+a_{65} & c_{15}^{\prime} & a_{54} & -a_{43}-a_{61} \\
c_{34} & a_{31}+a_{46} & c_{16}^{\prime} & a_{64}-a_{13} & c_{56}^{\prime} & -a_{53}
\end{array}
$$

Therefore the isotropy subalgebra $g_{X_{0}}$ at $X_{0}$ is given by

$$
\begin{aligned}
\mathfrak{g}_{X_{0}} & =\left\{\left(A,\left(\begin{array}{ll}
a & b \\
c & d
\end{array}\right)\right) \in \mathfrak{g} \mathfrak{I}(6) \oplus \mathfrak{g} \mathfrak{l}(2) \mid c_{i j}=c_{i j}^{\prime}=0 \text { in }(5.9)\right\} \\
& =\left\{\left(\frac{\alpha}{\gamma} \mid \frac{\beta}{-\alpha}\right) \oplus(0) \mid \alpha, \beta, \gamma \text { are diagonal } 3 \times 3 \text { matrices }\right\} \\
& \cong \mathscr{I}(2) \oplus \mathfrak{g}(2) \oplus \mathscr{g}(2) .
\end{aligned}
$$

Since $\operatorname{dim} \mathrm{g}_{X_{0}}=9=\operatorname{dim} S L(6) \times G L(2)-\operatorname{dim} V(15) \otimes V(2)$, it is a P.V., and by Proposition 25 in $\S 4$, it is regular.

The discriminant $f(X)$ of the binary cubic form $F_{X}(u, v)\left(=P f f\left(u X_{1}\right.\right.$ $\left.+v X_{2}\right)$ ) is a relative invariant of degree 12 (see Proposition 6). We shall show that $f(X)$ is irreducible.

Put

$$
J=\left(\begin{array}{lll}
J_{1} & & 0 \\
& J_{1} & \\
0 & & J_{1}
\end{array}\right) \quad \text { where } J_{1}=\left(\begin{array}{l}
-1 \\
1
\end{array}\right) .
$$

Let $X$ be a $3 \times 3$ quaternion matrix. In general a quaternion can be represented as a $2 \times 2$ matrix $\left(\begin{array}{rr}x & -y \\ \bar{y} & \bar{x}\end{array}\right)$ and hence we can consider $X$ as a $6 \times 6$ matrix. In this case, if $X$ is a quaternion hermitian i.e. ${ }^{t} \bar{X}=$ $X$, then ${ }^{t}(X J)=-(X J)$. This gives a one-to-one correspondence from $3 \times 3$ quaternion hermitian matrices to $6 \times 6$ skew-symmetric matrices. Moreover we can define the determinant of quaternion hermitian matrices which corresponds to the Pfaffian of skew-symmetric matrices. Therefore $f(X)$ can be considered as a discriminant of the binary cubic form $\operatorname{det}\left(u X_{1}+v X_{2}\right)$ where $X_{1}, X_{2}$ are $3 \times 3$ quaternion hermitian matrices. 
We have seen in (10) that the restriction of $f(X)$ to $X=\left(X_{1}, X_{2}\right)$ where $X_{1}, X_{2}$ are $3 \times 3$ symmetric matrices over $C$, is irreducible and hence $f(X)$ itself must be irreducible.

Proposition 12. A triplet $\left(S L(2 m) \times G L(2), \Lambda_{2} \otimes \Lambda_{1}, V(m(2 m-1))\right.$ $\otimes V(2))$ is not a P.V. if $m \geqq 4$. A triplet $\left(S L(6) \times G L(2), \Lambda_{2} \otimes \Lambda_{1}, V(15)\right.$ $\otimes V(2))$ is a regular P.V. and its generic isotropy subgroup is locally isomorphic to $S L(2) \times S L(2) \times S L(2)$. The relative invariant is of degree 12.

II) The case of $n=2 m+1$.

We identify $V=V(m(2 m+1)) \otimes V(2)$ with $\left\{\left(X_{1}, X_{2}\right) \in M(2 m+1) \oplus\right.$ $\left.\left.M(2 m+1)\right|^{t} X_{1}=-X_{1},{ }^{t} X_{2}=-X_{2}\right\}$. The subgroups $G L(2 m+1)=S L(2 m$ $+1) \times G L(1)$ of $S L(2 m+1) \times G L(2)$ acts on $V$ as $X=\left(X_{1}, X_{2}\right) \mapsto$ $\left(A X_{1}^{t} \dot{A}, A X_{2}^{t} A\right)$ for $A \in G L(2 m+1)$. Let $X_{0}$ be a point

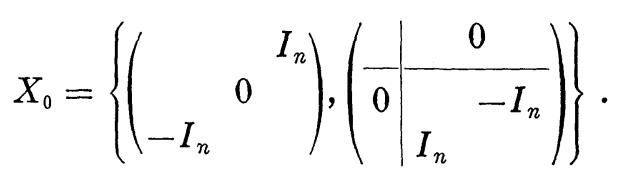

Then the isotropy subalgebra $g_{X_{0}}$ of $g l(2 m+1)$ at $X_{0}$ is

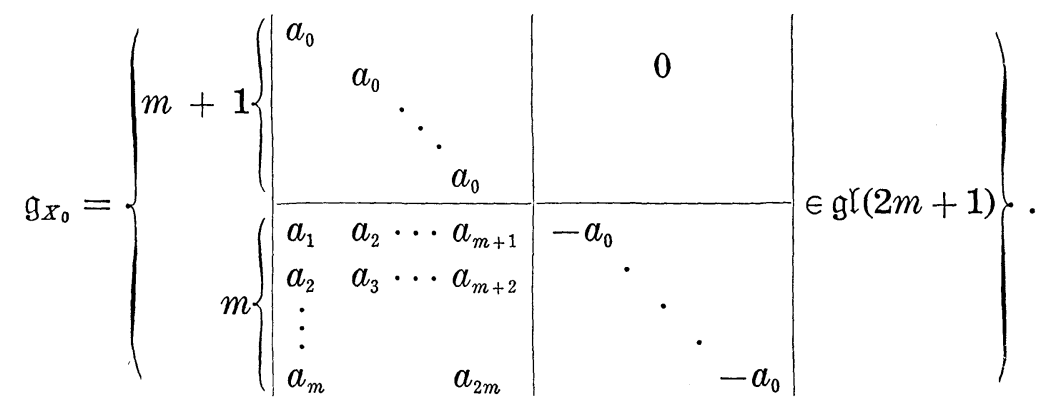

Since $\operatorname{dim} g_{X_{0}}=2 m+1=\operatorname{dim} \operatorname{gr}(2 m+1)-\operatorname{dim} V$, it is a P.V. and there is no relative invariant by (2) in Proposition 20 in $\S 4$. Hence $\left(S L(2 m+1) \times G L(2), \Lambda_{2} \otimes \Lambda_{1}, V(m(2 m+1)) \otimes V(2)\right)$ is a P.V. and there is no relative invariant.

Proposition 13. A triplet $\left(S L(2 m+1) \times G L(2), \Lambda_{2} \otimes \Lambda_{1}, V(m(2 m\right.$ $+1)) \otimes V(2))$ is a P.V. There is no relative invariant and hence it is not regular.

$$
\left(S L(5) \times G L(3), \Lambda_{2} \otimes \Lambda_{1}, V(10) \otimes V(3)\right) .
$$


Let $V_{1}$ be a vector space spanned by $u_{1}, \cdots, u_{5}$. Then $S L(5)$ acts on $V_{1}$ as $\rho_{1}(g)\left(u_{1}, \cdots, u_{5}\right)=\left(u_{1}, \cdots, u_{5}\right) g$ for $g \in S L(5)$. Let $V_{2}$ be a vector space spanned by 2-forms $u_{i} \wedge u_{j}(1 \leq i<j \leq 5)$. Then $S L(5)$ acts on $V_{2}$ as $\rho_{2}(g)\left(u_{i} \wedge u_{j}\right)=\rho_{1}(g) u_{i} \wedge \rho_{1}(g) u_{j}$ for $g \in S L(5)$. The infinitesimal action $d \rho_{2}$ of $\rho_{2}$ is given by $d \rho_{2}(A)\left(u_{i} \wedge u_{j}\right)=d \rho_{1}(A) u_{i} \wedge u_{j}+u_{k} \wedge d \rho_{1}(A) u_{j}$ for $A \in \mathfrak{g l}(5)$. Put $\omega_{1}=u_{1} \wedge u_{2}, \omega_{2}=u_{2} \wedge u_{3}, \omega_{3}=u_{1} \wedge u_{3}, \omega_{4}=u_{2} \wedge u_{4}$, $\omega_{5}=u_{1} \wedge u_{4}, u_{6}=u_{3} \wedge u_{4}, \omega_{7}=u_{4} \wedge u_{5}, \omega_{8}=u_{2} \wedge u_{5}, \omega_{9}=u_{3} \wedge u_{5}, \omega_{10}=$ $u_{1} \wedge u_{5}$. Then $\left\{\omega_{1}, \cdots, \omega_{10}\right\}$ is a basis of $V_{2}$, and for $A=\left(a_{i j}\right) \in \mathfrak{g l}(5)$, by simple calculation we have

$$
\begin{aligned}
& d \rho_{2}(A)\left(\omega_{1}, \cdots, \omega_{10}\right)=\left(\omega_{1}, \cdots, \omega_{10}\right)\left(A_{1} \mid A_{2}\right) \\
& A_{1}=\left|\begin{array}{c|c|c|c|c}
a_{1}+a_{2} & -a_{13} & a_{23} & -a_{14} & a_{24} \\
-a_{31} & a_{2}+a_{3} & a_{21} & a_{34} & 0 \\
a_{32} & a_{12} & a_{1}+a_{3} & 0 & a_{34} \\
-a_{41} & a_{43} & 0 & a_{2}+a_{4} & a_{21} \\
a_{42} & 0 & a_{43} & a_{12} & a_{1}+a_{4} \\
\hline 0 & -a_{42} & -a_{41} & a_{32} & a_{31} \\
0 & 0 & 0 & -a_{52} & -a_{51} \\
-a_{51} & a_{53} & 0 & a_{54} & 0 \\
0 & -a_{52} & -a_{51} & 0 & 0 \\
a_{52} & 0 & a_{53} & 0 & a_{54}
\end{array}\right| \\
& A_{2}=\left|\begin{array}{c|c|c|c|c}
0 & 0 & -a_{15} & 0 & a_{25} \\
-a_{24} & 0 & a_{35} & -a_{25} & 0 \\
-a_{14} & 0 & 0 & -a_{15} & a_{35} \\
a_{23} & -a_{25} & a_{45} & 0 & 0 \\
a_{13} & -a_{15} & 0 & 0 & a_{45} \\
\hline a_{3}+a_{4} & -a_{35} & 0 & a_{45} & 0 \\
-a_{53} & a_{4}+a_{5} & a_{42} & a_{43} & a_{41} \\
0 & a_{24} & a_{2}+a_{5} & a_{23} & a_{21} \\
a_{54} & a_{34} & a_{32} & a_{3}+a_{5} & a_{31} \\
0 & a_{14} & a_{12} & a_{13} & a_{1}+a_{5}
\end{array}\right|
\end{aligned}
$$

where $a_{i i}=a_{i}$ and $\sum a_{i}=0$.

We denote this matrix also by $d \rho_{2}(A)$, i.e., $d \rho_{2}(A) \in M(10)$. Identify $V=V(10) \otimes V(3)$ with $10 \times 3$ matrices $M(10,3)$. Then the action $\rho=\Lambda_{2}$ $\otimes \Lambda_{1}$ on $V$ is given by $\rho(g) X=\rho_{2}\left(g_{1}\right) X^{t} g_{2}$ for $g=\left(g_{1}, g_{2}\right) \in S L(5) \times G L(3)$, $X \in M(10,3)$. The infinitesimal action $d \rho$ of $\rho$ is given by $d \rho(\tilde{A}) X=$ $d \rho_{2}(A) X+X^{t} B$ for $\tilde{A}=(A, B)^{\prime} \in \mathfrak{g}=\mathfrak{g l}_{(}(5) \oplus \mathfrak{g} \mathfrak{g}(3)$. We shall calculate the isotropy subalgebra $g_{X_{0}}$ at $X_{0}={ }^{t}\left(I_{3} 0 \mid I_{3} 0\right) \in M(10,3)$. Then for $\tilde{A}=(A, B)$ with $A=\left(a_{i j}\right) \in \mathfrak{g l}(5), B=\left(b_{i j}\right) \in \operatorname{gr}(3)$, we have 


$$
d \rho(\tilde{A}) X_{0}=d \rho_{2}(A) X_{0}+X_{0}{ }^{t} B=\left|\begin{array}{c|c|c|c}
a_{1}+a_{2}+b_{1} & b_{21}-a_{13} & a_{23}-a_{15}+b_{31} \\
b_{12}-a_{31}-a_{24} & a_{2}+a_{3}+b_{2} & a_{21}+a_{35}+b_{32} \\
a_{32}-a_{14}+b_{13} & a_{12}+b_{23} & a_{1}+a_{3}+b_{3} \\
a_{23}-a_{41} & a_{43}-a_{25} & a_{45} \\
a_{42}+a_{13} & -a_{15} & a_{43} \\
a_{3}+a_{4}+b_{1} & b_{21}-a_{42}-a_{35} & b_{31}-a_{41} \\
b_{12}-a_{53} & a_{4}+a_{5}+b_{2} & a_{42}+b_{32} \\
b_{13}-a_{51} & a_{24}+a_{53}+b_{23} & a_{2}+a_{5}+b_{3} \\
a_{54} & a_{34}-a_{52} & a_{32}-a_{51} \\
a_{52} & a_{14} & a_{12}+a_{53}
\end{array}\right|
$$

where $d \rho_{2}(A)$ is given by (5.12).

Hence the isotropy subalgebra $\mathfrak{g}_{X_{0}}=\left\{\tilde{A} \mid d \rho(\tilde{A}) X_{0}=0\right\}$ is given as follows.

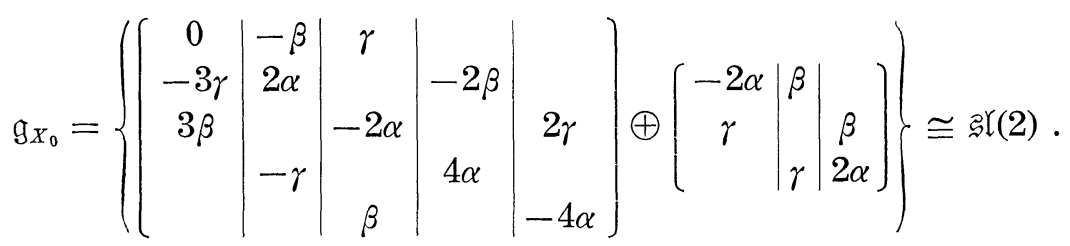

This is $4 \Lambda_{1} \oplus 2 \Lambda_{1}$ of $\mathfrak{g l}(2)$. Since $\operatorname{dim} \mathfrak{g}_{X_{0}}=3=\operatorname{dim} S L(5) \times G L(3)-$ $\operatorname{dim} V(10) \otimes V(3)$, it is a P.V. and moreover it is regular by Proposition 25 in $\S 4$.

Next we shall calculate the isotropy subalgebra $\mathfrak{g}_{X_{0}^{\prime}}$ at

$$
X_{0}^{\prime}={ }^{t}\left(\begin{array}{llllllllll}
1 & 0 & 0 & 0 & 0 & 0 & 0 & 0 & 0 & 0 \\
0 & 0 & 0 & 0 & 0 & 1 & 0 & 0 & 0 & 1 \\
0 & 1 & 0 & 0 & 0 & 0 & 1 & 0 & 0 & 0
\end{array}\right)
$$

Similarly, we have

$$
d \rho(\tilde{A}) X_{0}^{\prime}=\left|\begin{array}{c|c|c}
a_{1}+a_{2}+b_{1} & a_{25}+b_{21} & b_{31}-a_{13} \\
b_{13}-a_{31} & b_{23}-a_{24} & a_{2}+a_{3}+b_{3} \\
a_{32} & a_{35}-a_{14} & a_{12} \\
-a_{41} & a_{23} & a_{43}-a_{25} \\
a_{42} & a_{13}+a_{45} & -a_{15} \\
b_{12} & a_{3}+a_{4}+b_{2} & b_{32}-a_{42}-a_{35} \\
b_{13} & a_{41}-a_{53}+b_{23} & a_{4}+a_{5}+b_{3} \\
-a_{51} & a_{21} & a_{53}+a_{24} \\
0 & a_{31}+a_{54} & a_{34}-a_{52} \\
a_{52}+b_{12} & a_{1}+a_{5}+b_{2} & a_{14}+b_{32}
\end{array}\right| .
$$


Hence the isotropy subalgebra $g_{X_{0}^{\prime}}=\left\{\tilde{A} \mid d \rho(\tilde{A}) X_{0}^{\prime}=0\right\}$ at $X_{0}^{\prime}$ is given as follows.

$$
\begin{aligned}
& \mathfrak{g}_{X_{0}^{\prime}}=\left\{\tilde{A}=\left(\begin{array}{l|l|l|c}
2 \alpha+4 \beta & -2 \alpha-2 \beta & & \gamma \\
\alpha & & \\
\beta & -\delta \\
-\alpha-3 \beta
\end{array}\right)\right. \\
& \left.\oplus\left(\begin{array}{c}
-2 \beta \\
-\gamma \\
\delta
\end{array}|-\alpha-\beta| \begin{array}{c}
\alpha+2 \beta
\end{array}\right)\right\} \text {. }
\end{aligned}
$$

Since $\operatorname{dim} \mathfrak{g}-\operatorname{dim} \mathfrak{g}_{X_{0}^{\prime}}=33-4=29$, the orbit of $X_{0}^{\prime}$ is of codimension one. For $\tilde{A} \in \mathfrak{g}_{X_{0}^{\prime}}$, the $\operatorname{trace} \operatorname{tr}_{V} \tilde{A}$ on $V=V(10) \otimes V(3)$ is $10(-2 \beta-$ $(\alpha+\beta)+(\alpha+2 \beta))=-10 \beta$ and the trace $\operatorname{tr}$ ad $\mathfrak{g}_{X_{0}^{\prime}} \tilde{A}$ of the adjoint representation on $\mathfrak{g}_{x_{0}^{\prime}}$ is $5 \beta$. By the degree formula, i.e., Proposition 15 in $\S 4$, the degree of the relative invariant $f(x)$ is given by

$$
\operatorname{deg} f(x)=\frac{\operatorname{tr}_{V} \tilde{A}+\operatorname{tr} \operatorname{ad} g_{X_{0}^{\prime}} \tilde{A}}{\operatorname{tr}_{V} \tilde{A}} \cdot \operatorname{dim} V=\frac{-10 \beta+5 \beta}{-10 \beta} \times 30=15 .
$$

Proposition 14. A triplet $\left(S L(5) \times G L(3), \Lambda_{2} \otimes \Lambda_{1}, V(10) \otimes V(3)\right)$ is a regular $P . V$. and its generic isotropy subgroup is locally isomorphic to $S L(2)$. The relative invariant is of degree 15 .

$$
\left(S L(5) \times G L(4), \Lambda_{2} \otimes \Lambda_{1}, V(10) \otimes V(4)\right)
$$

We identify $V=V(10) \otimes V(4)$ with $10 \times 4$ matrices $M(10,4)$. Then the action of $\rho_{2}=\Lambda_{2} \otimes \Lambda_{1}$ is given by $\rho(g) X=\rho_{2}\left(g_{1}\right) X^{t} g_{2}$ for $g=\left(g_{1}, g_{2}\right)$ $\in G=S L(5) \times G L(4), X \in V$ where $\rho_{2}$ is defined as in (12). The infinitesimal action $d \rho$ of $\rho$ is given by $d \rho(\tilde{A}) X=d \rho_{2}(A) X+X^{t} B$ for $\tilde{A}=$ $(A, B) \in \mathfrak{g}=\operatorname{gl}(5) \oplus \mathfrak{g l}(4)$ where $d \rho_{2}(A)$ is the $10 \times 10$ matrix of (5.12). We shall calculate the isotropy subalgebra $\mathfrak{g}_{X_{0}}$ at $X_{0}={ }^{t}\left(I_{4} 0 \mid I_{4} 0\right) \in M(10,4)$. Similarly as in (12), we have

$$
d \rho(\tilde{A}) X_{0}=d \rho_{2}(A) X_{0}+X_{0}^{t} B
$$




$$
=\left|\begin{array}{c|c|c|c}
a_{1}+a_{2}+b_{1} & b_{21}-a_{13} & a_{23}-a_{15}+b_{31} & b_{41}-a_{14} \\
b_{12}-a_{31}-a_{24} & a_{2}+a_{3}+b_{2} & a_{21}+a_{35}+b_{32} & a_{34}-a_{25}+b_{42} \\
a_{32}-a_{14}+b_{13} & a_{12}+b_{23} & a_{1}+a_{3}+b_{3} & b_{43}-a_{15} \\
a_{23}-a_{41}+b_{14} & a_{43}-a_{25}+b_{24} & a_{45}+b_{34} & a_{2}+a_{4}+b_{4} \\
a_{42}+a_{13} & -a_{15} & a_{43} & a_{12} \\
a_{3}+a_{4}+b_{1} & b_{21}-a_{42}-a_{35} & b_{31}-a_{41} & a_{32}+a_{45}+b_{41} \\
b_{12}-a_{53} & a_{4}+a_{5}+b_{2} & a_{42}+b_{32} & a_{43}-a_{52}+b_{42} \\
b_{13}-a_{51} & a_{24}+a_{53}+b_{23} & a_{2}+a_{5}+b_{3} & a_{54}+a_{23}+b_{43} \\
b_{14}+a_{54} & a_{34}-a_{52}+b_{24} & a_{32}-a_{51}+b_{34} & a_{3}+a_{5}+b_{4} \\
a_{52} & a_{14} & a_{12}+a_{53} & a_{13}
\end{array}\right|
$$

Hence the isotropy subalgebra $\mathfrak{g}_{X_{0}}=\left\{\tilde{A} \in \mathfrak{g} \mid d \rho(\tilde{A}) X_{0}=0\right\}$ at $X_{0}$ is zero. Since $\operatorname{dim} \mathfrak{g}_{X_{0}}=0=\operatorname{dim} S L(5) \times G L(4)-\operatorname{dim} V(10) \otimes V(4)$, it is a regular P.V.

Since $\operatorname{dim} G=\operatorname{dim} V$, there is a relative invariant polynomial of degree 40 by Proposition 16 in $\S 4$.

Similarly one can check that the isotropy subalgebra $g_{x_{0}^{\prime}}$ at

$$
X_{0}^{\prime}=\left(\omega_{7}, \omega_{3}+\omega_{8}, \omega_{2}+\omega_{5}, \omega_{4}+\omega_{10}\right)=\left(\begin{array}{llllllllll}
0 & 0 & 0 & 0 & 0 & 0 & 1 & 0 & 0 & 0 \\
0 & 0 & 1 & 0 & 0 & 0 & 0 & 1 & 0 & 0 \\
0 & 1 & 0 & 0 & 1 & 0 & 0 & 0 & 0 & 0 \\
0 & 0 & 0 & 1 & 0 & 0 & 0 & 0 & 0 & 1
\end{array}\right)
$$

is of one-dimension, i.e., the orbit of $X_{0}^{\prime}$ is of codimension one. Hence by Proposition 16 in $\S 4$, the relative invariant of degree 40 is irreducible. This point $X_{0}^{\prime}$ was found by I. Ozeki ([20]).

Proposition 15. A triplet $\left(S L(5) \times G L(4), \Lambda_{2} \otimes \Lambda_{1}, V(10) \otimes V(4)\right)$ is a regular $P . V$. and its generic isotropy subgroup is a finite group. The relative invariant is of degree 40 .

$$
\left(S L(n) \times S L(n) \times G L(2), \Lambda_{1} \otimes \Lambda_{1} \otimes \Lambda_{1}, V(n) \otimes V(n) \otimes V(2)\right)
$$

$$
(n \geqq 3) \text {. }
$$

We identify $V(n) \otimes V(n) \otimes V(2)$ with $V=M(n) \oplus M(n)$. Then the action $\rho=\Lambda_{1} \otimes \Lambda_{1} \otimes \Lambda_{1}$ is given by $\rho(g) X=\left(A\left(\alpha X_{1}+\beta X_{2}\right)^{t} B, A\left(\gamma X_{1}+\right.\right.$ .$\left.\left.\delta X_{2}\right)^{t} B\right)$ where $g=\left(A, B,\left(\begin{array}{ll}\alpha & \beta \\ \gamma & \delta\end{array}\right)\right) \in S L(n) \times S L(n) \times G L(2)$ and $X=\left(X_{1}\right.$, $\left.X_{2}\right) \in V$. For each $X=\left(X_{1}, X_{2}\right)$ in $V$, we can obtain a binary $n$-form $F_{X}(u, v)=\operatorname{det}\left(u X_{1}+v X_{2}\right)$ which is invariant under the action of $S L(n)$ $\times S L(n)$. Clearly the map $\varphi: X \mapsto F_{X}(u, v)$ is equivariant. Moreover, it is generically surjective since $\varphi(X)=\left(u-\lambda_{1} v\right) \cdots\left(u-\lambda_{n} v\right)$ for 


$$
X=\left(I_{n},\left(\begin{array}{lll}
-\lambda_{1} & & \\
& \ddots & \\
& & -\lambda_{n}
\end{array}\right)\right)
$$

in $V$. Therefore by Lemma 5 in $\S 2$, if $n \geqq 4$, then it is not a P.V. since $\left(G L(2), n \Lambda_{1}, V(n+1)\right)$ is not a P.V. for $n \geqq 4$. Assume that $n=$ 3. The infinitesimal representation $d \rho$ of $\rho$ is given by

$$
\begin{aligned}
& d \rho\left(A, B,\left(\begin{array}{ll}
a & b \\
c & d
\end{array}\right)\right) X \\
& \quad=\left(A X_{1}+X_{1}^{t} B+a X_{1}+b X_{2}, A X_{2}+X_{2}^{t} B+c X_{1}+d X_{2}\right)
\end{aligned}
$$

for $\left(A, B,\left(\begin{array}{ll}a & b \\ c & d\end{array}\right)\right) \in \mathfrak{g l}(n) \oplus \mathfrak{g l}(n) \oplus \mathfrak{g l}(2)$. Hence for

$$
X_{0}=\left\{\left(\begin{array}{lll}
1 & & \\
& 1 & \\
& & 1
\end{array}\right),\left(\begin{array}{lll}
1 & & \\
& 0 & \\
& & -1
\end{array}\right)\right\} \text {, }
$$

we have

$$
\begin{aligned}
d \rho\left(A, B,\left(\begin{array}{ll}
a & b \\
c & d
\end{array}\right)\right) X_{0}= & \left(\left(\begin{array}{c|c|c}
a+b+a_{11}+b_{11} & a_{12}+b_{21} & a_{13}+b_{31} \\
a_{21}+b_{12} & a+a_{22}+b_{22} & a_{23}+b_{32} \\
a_{31}+b_{13} & a_{32}+b_{23} & a-b+a_{33}+b_{33}
\end{array}\right)\right. \\
& \left.\left(\begin{array}{c|c|c}
c+d+a_{11}+b_{11} & b_{21} & -a_{13}+b_{31} \\
a_{21} & c & -a_{23} \\
a_{31}-b_{13} & -b_{23} & c-d-a_{33}-b_{33}
\end{array}\right)\right)
\end{aligned}
$$

where $A=\left(a_{i j}\right), B=\left(b_{i j}\right) \in \mathfrak{g l}(3),\left(\begin{array}{ll}a & b \\ c & d\end{array}\right) \in \mathfrak{g} \mathfrak{l}(2)$.

Therefore the isotropy subalgebra

$$
\begin{aligned}
& \mathfrak{g}_{X_{0}}=\left\{\left(A, B,\left(\begin{array}{ll}
a & b \\
c & d
\end{array}\right)\right) \in \mathfrak{I l}(3) \oplus \mathfrak{I l}(3) \oplus \mathfrak{g l}(2) \mid d \rho\left(A, B,\left(\begin{array}{ll}
a & b \\
c & d
\end{array}\right)\right) X_{0}=0\right\} \\
& =\left\{\left(\left(\begin{array}{lll}
\alpha & & \\
& \beta & \\
& & \gamma
\end{array}\right)\left(\begin{array}{ccc}
-\alpha & & \\
& -\beta & \\
& & -\gamma
\end{array}\right)(0)\right) \mid \alpha+\beta+\gamma=0\right\} \cong \operatorname{gl}(1) \oplus \mathfrak{g l}(1) \text {. }
\end{aligned}
$$

Since $\operatorname{dim} \mathfrak{g}_{X_{0}}=2=\operatorname{dim} S L(3) \times S L(3) \times G L(2)-\operatorname{dim} V$, it is a regular P.V. by Proposition 25 in $\S 4$. The discriminant $f(X)$ of the binary cubic form $F_{X}(u, v)=\operatorname{det}\left(u X_{1}+v X_{2}\right)$ is a relative invariant of degree 12 . This is irreducible since we have seen in (10) that the restriction of $f(X)$ to $\left\{X=\left(X_{1}, X_{2}\right) \in M(3) \oplus M(3) \mid{ }^{t} X_{1}=X_{1},{ }^{t} X_{2}=X_{2}\right\}$ is irreducible. 
Proposition 16. A triplet $\left(S L(n) \times S L(n) \times G L(2), \Lambda_{1} \otimes \Lambda_{1} \otimes \Lambda_{1}, V(n)\right.$ $\otimes V(n) \otimes V(2))$ is not a P.V. if $n \geqq 4 . \quad$ A triplet $(S L(3) \times S L(3) \times G L(2)$, $\left.\Lambda_{1} \otimes \Lambda_{1} \otimes \Lambda_{1}, V(3) \otimes V(3) \otimes V(2)\right)$ is a regular $P . V$. and its generic isotropy subgroup is locally isomorphic to $G L(1) \times G L(1)$. The relative invariant is of degree 12.

$$
\left(S p(n) \times G L(m), \Lambda_{1} \otimes \Lambda_{1}, V(2 n) \otimes V(m)\right) \quad(n \geqq m \geqq 1) .
$$

We identify $V=V(2 n) \otimes V(m)$ with $2 n \times m$ matrices $M(2 n, m)$. Then the action $\rho=\Lambda_{1} \otimes \Lambda_{1}$ is given by $\rho(g) X=g_{1} X^{t} g_{2}$ where $X \in M(2 n, m)$ and $g=\left(g_{1}, g_{2}\right) \in S p(n) \times G L(m)$. Let $g=\xi \mathfrak{s}(n) \oplus g l(m)$ be the Lie algebra of $S p(n) \times G L(m)$. Then the infinitesimal action $d \rho$ of $\rho$ is given by $d \rho(A) X=A_{1} X+X^{t} A_{2}$ where $A=\left(A_{1}, A_{2}\right) \in \mathfrak{g}=\mathfrak{g p}(n) \oplus \mathfrak{g l}(m)$.

I) The case when $m$ is even, i.e., $m=2 \ell(n \geqq 2 \ell \geqq 2)$. We shall calculate the isotropy subalgebra $g_{X_{0}}$ at

$$
X_{0}={ }^{t}\left(\begin{array}{cc|c}
I_{\ell} & 0 & 0 \\
\hline 0 & \frac{0}{I_{\ell}} & 0
\end{array}\right) \in M(2 n, 2 \ell)
$$

where $I_{\ell}$ denotes the identity matrix of size $\ell$. An element $A$ of $g$ can be written as follows.

$$
A=\left(\begin{array}{cc|cc}
A_{1} & A_{2} & B_{1} & B_{2} \\
A_{3} & A_{4} & { }^{t} B_{2} & B_{4} \\
\hline C_{1} & C_{2} & -{ }^{t} A_{1} & -{ }^{t} A_{3} \\
{ }^{t} C_{2} & C_{4} & -{ }^{t} A_{2} & -{ }^{t} A_{4}
\end{array}\right) \oplus\left(\frac{D_{1}}{D_{3}} \mid \frac{D_{2}}{D_{4}}\right)
$$

where $A_{1}, B_{1}, C_{1}, D_{j} \in M(\ell)$

for $1 \leq j \leq 4 ; A_{2}, B_{2}, C_{2} \in M(\ell, n-\ell) ; A_{3} \in M(n-\ell, \ell) ; A_{4}, B_{4}, C_{4} \in M(n-\ell)$ and ${ }^{t} B_{1}=B_{1},{ }^{t} B_{4}=B_{4},{ }^{t} C_{1}=C_{1},{ }^{t} C_{4}=C_{4}$. Then we have

$$
\begin{aligned}
d \rho(A) X_{0} & =\left(\begin{array}{cc|cc}
A_{1} & A_{2} & B_{1} & B_{2} \\
A_{3} & A_{4} & { }^{t} B_{2} & B_{4} \\
C_{1} & C_{2} & -{ }^{t} A_{1} & -{ }^{t} A_{3} \\
{ }^{t} C_{2} & C_{4} & -{ }^{t} A_{2} & -{ }^{t} A_{4}
\end{array}\right)\left(\begin{array}{c|c}
I_{\ell} & 0 \\
0 & 0 \\
0 & I_{\ell} \\
0
\end{array}\right)+\left(\begin{array}{cc}
I_{\ell} & 0 \\
0 & 0 \\
0 & I_{\ell} \\
0 & 0
\end{array}\right)\left(\begin{array}{cc}
{ }^{t} D_{1} & { }^{t} D_{3} \\
{ }^{t} D_{2} & { }^{t} D_{4}
\end{array}\right) \\
& =\left(\begin{array}{ccc}
A_{1}+{ }^{t} D_{1} & B_{1}+{ }^{t} D_{3} \\
A_{3} & { }^{t} B_{2} \\
C_{1}+{ }^{t} D_{2} & -{ }^{t} A_{1}+{ }^{t} D_{4} \\
{ }^{t} C_{2} & -{ }^{t} A_{2}
\end{array}\right) \cdot
\end{aligned}
$$

Hence the isotropy subalgebra $\mathfrak{g}_{X_{0}}=\left\{A \in \mathfrak{g} \mid d \rho(A) X_{0}=0\right\}$ is given as follows. 


$$
\mathfrak{g}_{X_{0}}=\left\{\left(\begin{array}{cc|cc}
A_{1} & 0 & B_{1} & 0 \\
0 & A_{4} & 0 & B_{4} \\
\hline C_{1} & 0 & -{ }^{t} A_{1} & 0 \\
0 & C_{4} & 0 & -{ }^{t} A_{4}
\end{array}\right] \oplus\left(-\left(\begin{array}{cc}
A_{1} & B_{1} \\
C_{1} & -{ }^{t} A_{1}
\end{array}\right)\right)\right\} \cong \mathfrak{g p}(\ell) \oplus \mathfrak{g p}(n-\ell) .
$$

Since $\operatorname{dim} \mathfrak{g}_{X_{0}}=\ell(2 \ell+1)+(n-\ell)(2 n-2 \ell+1)=\left\{n(2 n+1)+4 \ell^{2}\right\}-4 n \ell$ $=\operatorname{dim} S p(n) \times G L(2 \ell)-\operatorname{dim} V(2 n) \otimes V(2 \ell)$, this triplet is a regular P.V. by Proposition 25 in $\S 4$.

The relative invariant $f(X)$ is given by $P f f\left({ }^{t} X J X\right)$ where

$$
J=\left(\begin{array}{c|c}
0 & I_{n} \\
\hdashline-I_{n} & 0
\end{array}\right),
$$

and Pff denotes the Pfaffian of the $2 \ell \times 2 \ell$ skew-symmetric matrix ${ }^{t} X J X$. Since $S p(n)=\left\{g_{1} \in G L(2 n) \mid{ }^{t} g_{1} J g_{1}=J\right\}$, we have $f(\rho(g) X)=f\left(g_{1} X^{t} g_{2}\right)=$ $P f f\left(g_{2}{ }^{t} X^{t} g_{1} J g_{1} X^{t} g_{2}\right)=\operatorname{Pff}\left(g_{2}{ }^{t} X J X^{t} g_{2}\right)=\operatorname{det} g_{2} \cdot \operatorname{Pff}\left({ }^{t} X J X\right)=\operatorname{det} g_{2} \cdot f f(X)$ for $g=\left(g_{1}, g_{2}\right) \in S p(n) \times G L(2 \ell)$. By Proposition 18 in $\S 4$, the degree of any relative invariant is multiple of $2 \ell$. Since $\operatorname{deg} f(X)=2 \ell, f(X)$ is irreducible.

Proposition 17. A triplet $\left(S p(n) \times G L(2 m), \Lambda_{1} \otimes \Lambda_{1}, V(2 n) \otimes V(2 m)\right)$ $(n \geqq 2 m \geqq 2)$ is a regular $P . V$. and its generic isotropy subgroup is locally isomorphic to $S p(m) \times S p(n-m)$. The relative invariant is of degree $2 m$.

Note that this proposition holds even if $2 n \geqq 2 m>n$, but in this case, it is not reduced.

II) The case when $m$ is odd, i.e., $m=2 \ell+1$.

We shall calculate the isotropy subalgebra $g_{X_{0}}$ at

$$
X_{0}={ }^{t}\left(\begin{array}{cc|c}
I_{\ell} & 0 & 0 \\
\hline 0 & I_{\ell+1} & 0
\end{array}\right) \in M(2 n, 2 \ell+1) .
$$

An element $A$ of $\mathrm{g}$ can be written as follows.

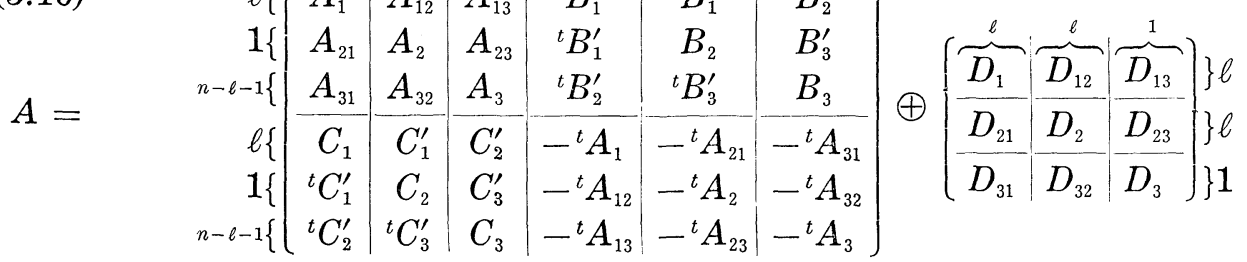


Then we have

(5.17) $d \rho(A) X_{0}=$

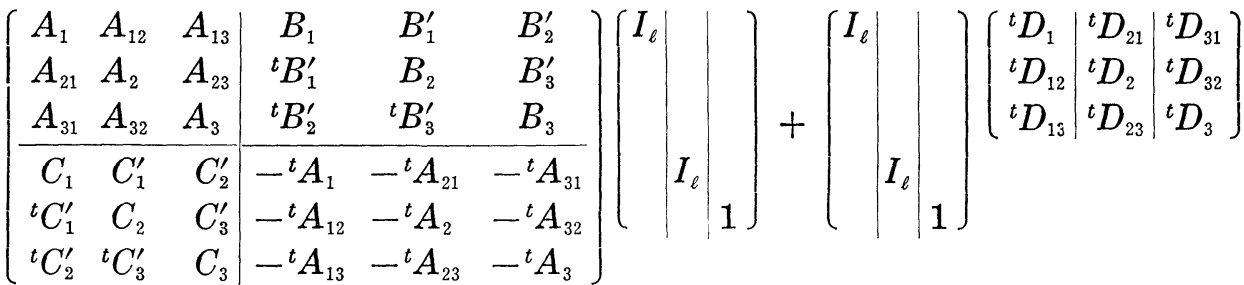

$$
\begin{aligned}
& =\left(\begin{array}{c|c|c}
A_{1}+{ }^{t} D_{1} & B_{1}+{ }^{t} D_{21} & B_{1}^{\prime}+{ }^{t} D_{31} \\
A_{21} & { }^{t} B_{1}^{\prime} & B_{2} \\
A_{31} & { }^{t} B_{2}^{\prime} & { }^{t} B_{3}^{\prime} \\
C_{1}+{ }^{t} D_{12} & -{ }^{t} A_{1}+{ }^{t} D_{2} & -{ }^{t} A_{21}+{ }^{t} D_{32} \\
{ }^{t} C_{1}^{\prime}+{ }^{t} D_{13} & -{ }^{t} A_{12}+{ }^{t} D_{23} & -{ }^{t} A_{2}+{ }^{t} D_{3} \\
{ }^{t} C_{2}^{\prime} & -{ }^{t} A_{13} & -{ }^{t} A_{23}
\end{array}\right)
\end{aligned}
$$

and hence the isotropy subalgebra $\mathfrak{g}_{X_{0}}=\left\{A \in \mathfrak{g} \mid d \rho(A) X_{0}=0\right\}$ is given as follows.

$$
\begin{aligned}
& g_{X_{0}}=\left\{\left(\begin{array}{ccc|ccc}
A_{1} & A_{12} & 0 & B_{1} & 0 & 0 \\
0 & A_{2} & 0 & 0 & 0 & 0 \\
0 & A_{32} & A_{3} & 0 & 0 & B_{3} \\
\hline C_{1} & C_{1}^{\prime} & 0 & -{ }^{t} A_{1} & 0 & 0 \\
{ }^{t} C_{1}^{\prime} & C_{2} & C_{3}^{\prime} & -{ }^{t} A_{12} & -{ }^{t} A_{2} & -{ }^{t} A_{32} \\
0 & { }^{t} C_{3}^{\prime} & C_{3} & 0 & 0 & -{ }^{t} A_{3}
\end{array}\right) \oplus\left(-\left(\begin{array}{c|c|c|c}
A_{1} & B_{1} & 0 \\
C_{1} & -{ }^{t} A_{1} & 0 \\
\hline C_{1}^{\prime} & -{ }^{t} A_{12} & -{ }^{t} A_{2}
\end{array}\right)\right)\right. \\
& \left.\cong\left\{\begin{array}{c|c|c|c|c}
-{ }^{t} A_{2} & { }^{t} C_{1}^{\prime}-{ }^{t} A_{12} & C_{3}^{\prime}-{ }^{t} A_{32} & C_{2} \\
\hline 0 & A_{1} B_{1} & 0 & A_{12} \\
\hline 0 & C_{1}-{ }^{t} A_{1} & 0 & C_{1}^{\prime} \\
\hline 0 & 0 & A_{3} B_{3} & A_{32} \\
\hline 0 & 0 & \frac{C_{3}-{ }^{t} A_{3}}{{ }^{t} C_{3}^{\prime}} \\
\hline 0 & \frac{A_{2}}{A^{\prime}}
\end{array}\right\} \oplus\left(-\left(\begin{array}{cc|c}
A_{1} & B_{1} & 0 \\
C_{1}-{ }^{t} A_{1} & \\
\hline C_{1}^{\prime}-{ }^{t} A_{12} & -{ }^{t} A_{2}
\end{array}\right)\right)\right\} \\
& \cong(\mathfrak{g l}(1) \oplus \mathfrak{Z} \mathfrak{p}(\ell) \oplus \mathfrak{Z} \mathfrak{p}(n-\ell-1)) \oplus \mathfrak{u}(2 n-1)
\end{aligned}
$$

where $\mathfrak{u}(2 n-1)$ is the nilpotent Lie algebra of dimension $(2 n-1)$. The first isomorphism is obtained by changing rows and columns from $\{1, \cdots, 6\}$ to $\{5,1,4,3,6,2\}$. Since

$$
\begin{aligned}
\operatorname{dim} \mathfrak{g}_{X_{0}} & =1+\ell(2 \ell+1)+(n-\ell-1)(2 n-2 \ell-1)+(2 n-1) \\
& =n(2 n+1)+(2 \ell+1)^{2}-2 n(2 \ell+1)
\end{aligned}
$$




$$
=\operatorname{dim} S p(n) \times G L(2 \ell+1)-\operatorname{dim} V
$$

it is a P.V. Since $g_{X_{0}} \not \subset \mathfrak{g r}(V)$, by (2) in Proposition 20 in $\S 4$, there is no relative invariant. In particular, it is not regular.

Proposition 18. A triplet $\left(S p(n) \times G L(2 m+1), \Lambda_{1} \otimes \Lambda_{1}, V(2 n) \otimes\right.$ $V(2 m+1))(n \geqq 2 m+1 \geqq 1)$ is a $P . V$. and its generic isotropy subgroup is locally isomorphic to $(G L(1) \times S p(m) \times S p(n-m)) \cdot U(2 n-1)$ where $U(2 n-1)$ is a unipotent group of dimension $(2 n-1)$. There is no relative invariant and hence it is not regular.

$$
\left(G L(1) \times S p(n) \times S O(m), \square \otimes \Lambda_{1} \otimes \Lambda_{1}, V(1) \otimes V(2 n) \otimes V(m)\right)
$$

$$
(2 n \geqq m \geqq 3)
$$

By Proposition 13 in $\S 2$, this triplet is a P.V. if and only if $\left(G L(1) \times S O(m), \square \otimes \Lambda_{2}, V(1) \otimes V\left(\frac{1}{2} m(m-1)\right)\right)$ is a P.V. Since $\Lambda_{2}$ is the adjoint representation of $S O(m)$, it is a P.V. only when rank $S O(m)=1$, i.e., $m=3$, by Proposition 2. Assume that $m=3$. We identify $V=$ $V(1) \otimes V(2 n) \otimes V(3)$ with $2 n \times 3$ matrices $M(2 n, 3)$. Then the action $\rho=\square \otimes \Lambda_{1} \otimes \Lambda_{1}$ is given by $\rho(g) X=c A X^{t} B$ where $g=(c, A, B) \in G L(1)$ $\times S p(n) \times S O(m)$ and $X \in M(2 n, 3)$. Let $g$ be the Lie algebra of $G L(1)$ $\times S p(n) \times S O(m)$ i.e.

$$
\mathfrak{g}=\left\{(d) \oplus\left[\begin{array}{c:c}
A & B \\
\hdashline C & -{ }^{t} A
\end{array}\right] \oplus\left[\begin{array}{rrr}
0 & a & b \\
-b & c & 0 \\
-a & 0 & -c
\end{array}\right] \mid \begin{array}{l}
A, B, C \in M(n) \\
{ }^{t} B=B,{ }^{t} C=C
\end{array}\right\}
$$

The infinitesimal action $d \rho$ of $\rho$ is given by $d \rho(d, A, B) X=d X+A X+$ $X^{t} B$ for $(d, A, B) \in$ g. Put

$$
X_{0}=\left(\begin{array}{lll|ll}
1 & 0 & & \\
0 & 1 & 0 & & 0 \\
0 & 0 & & 01 &
\end{array}\right) \in V=M(2 n, 3)
$$

Then we have

(5.18) $d \rho(d, A, B) X_{0}$ 


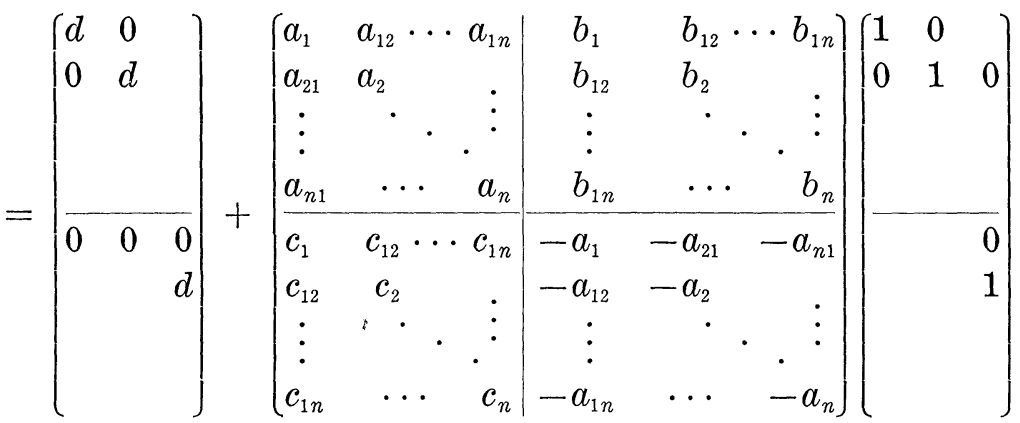

$$
\begin{aligned}
& +\left[\begin{array}{rrr}
1 & 0 & \\
0 & 1 & 0 \\
& & \\
\hline & & 0 \\
& & 1
\end{array}\right]\left(\begin{array}{rrr}
0 & -b & -a \\
a & c & 0 \\
b & 0 & -c
\end{array}\right) \\
& =\left(\begin{array}{lllllr}
a_{1}+d & a_{21}+a & a_{31} \cdots a_{n 1} & c_{1} & c_{12}+b & c_{13} \cdots c_{1 n} \\
a_{12}-b & a_{2}+d+c & a_{32} \cdots a_{n 2} & c_{12} & c_{2} & c_{23} \cdots c_{2 n} \\
b_{12}-a & b_{2} & b_{23} \cdots b_{2 n} & -a_{21} & -a_{2}+d-c & -a_{23} \cdots-a_{2 n}
\end{array}\right)
\end{aligned}
$$

Hence the isotropy subalgebra $\mathfrak{g}_{X_{0}}=\left\{(d, A, B) \mid d \rho(d, A, B) X_{0}=0\right\}$ at $X_{0}$ is given by

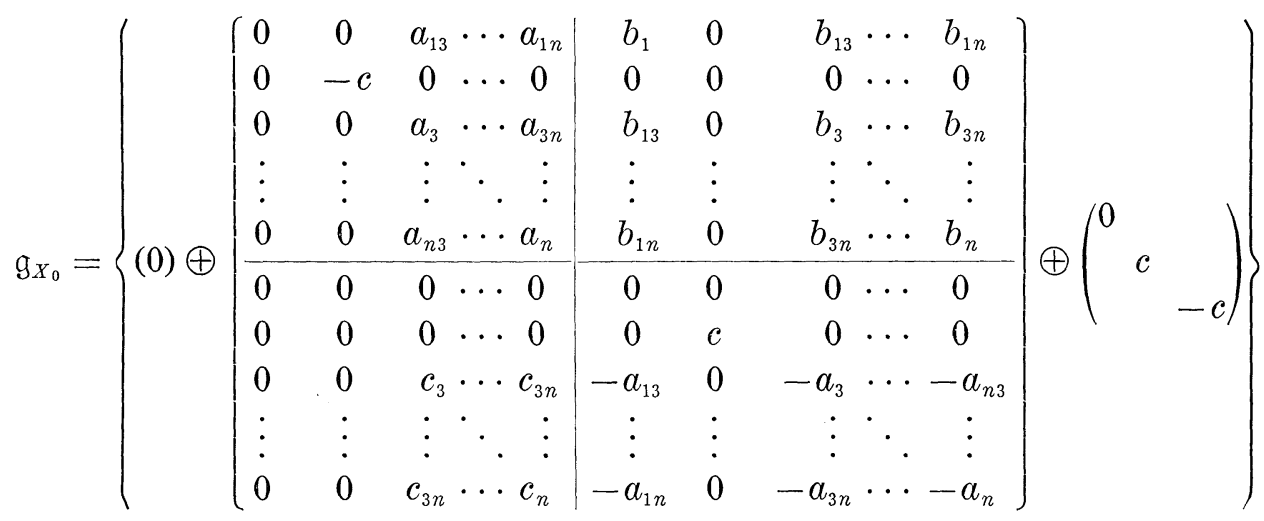

Since $\operatorname{dim} g_{X_{0}}=2 n^{2}-5 n+4=\operatorname{dim} G L(1) \times S p(n) \times S O(3)-\operatorname{dim} M(2 n, 3)$, this triplet is a P.V.

However, its generic isotropy subgroup is not reductive since it is locally isomorphic to the semi-direct product of $S p(n-2) \times S O(2)$ with 
a unipotent group $U(2 n-3)$ of dimension $(2 n-3)$.

By Corollary 23 in $\S 4$, this P.V. is not regular. Since $\mathfrak{g}_{x_{0}} \subset \operatorname{sl}(V)$, there exists a relative invariant $f(x)$ by (3) in Proposition 20 in $\S 4$. In fact, a polynomial $f(x)=\operatorname{tr}\left({ }^{t} X J X K\right)^{2}$ is a relative invariant where $S p(n)=\left\{\left.A \in G L(2 n)\right|^{t} A J A=J\right\}$ and $S O(m)=\left\{\left.B \in S L(m)\right|^{t} B K B=K\right\}$ since $f\left(A X^{t} B\right)=\operatorname{tr}\left(B^{t} X^{t} A J A X^{t} B K\right)^{2}=\operatorname{tr}\left(B^{t} X J X K B^{-1}\right)^{2}=\operatorname{tr}\left({ }^{t} X J X K\right)^{2}=f(X)$ for $A \in S p(n), B \in S O(m)$. Since $f\left(X_{0}\right)=2, f(X)$ is not identically zero. Note that $\operatorname{tr}^{t} X J X K$ is identically zero.

Since this P.V. is not regular, the Hessian of $f(X)=\operatorname{tr}\left({ }^{t} X J X K\right)^{2}$ must be identically zero. We can check this directly as follows.

The infinitesimal character $\delta \chi$ of $f(X)$ is given by $\delta \chi(\tilde{A})=4 d$ for $\tilde{A}=(d, A, B) \in \mathfrak{g} . \quad$ By Proposition 10 in $\S 4$, Hessian $H_{f}(x)$ of $f(x)$ is not identically zero if and only if $\operatorname{grad} \log f: V-S \rightarrow V^{*}$ is generically surjective. In view of (1) in Proposition 9 in $\S 1$, the $\operatorname{map} \operatorname{grad} \log f$ is generically surjective if and only if $\operatorname{grad} \log f\left(X_{0}\right)$ is a generic point of the dual P.V. By the inner product $\langle X, Y\rangle=\operatorname{tr}^{t} X Y$ for $X, Y \in$ $M(2 n, 3)$, we may identify this P.V. with its dual. By (2) in Proposition 9 in $\S 1$, we have $\left\langle d \rho(d, A, B) X_{0}\right.$, grad $\left.\log f\left(X_{0}\right)\right\rangle=\delta \chi(d, A, B)=4 d$ for any $(d, A, B) \in \mathrm{g}$. This condition completely characterizes grad $\log f\left(X_{0}\right)$ since $\left\{d \rho(d, A, B) X_{0} \mid(d, A, B) \in \mathfrak{g}\right\}=M(2 n, 3)$.

From (5.18), we have

$$
\operatorname{grad} \log f\left(X_{0}\right)=\left(\begin{array}{ccc|ccc}
0 & 0 & & & \\
0 & 2 & 0 & & & 0 \\
0 & 0 & & 0 & 2 &
\end{array}\right) .
$$

Since the rank of this $2 n \times 3$ matrix is 2 , it cannot be a generic point and hence the Hessian of $f(X)$ is identically zero.

Proposition 19. A triplet $\left(G L(1) \times S p(n) \times S O(m), \square \otimes \Lambda_{1} \otimes \Lambda_{1}, V(1)\right.$ $\otimes V(2 n) \otimes V(m))$ is not a P.V. for $2 n \geqq m \geqq 4$. A triplet $(G L(1) \times S p(n)$ $\left.\times S O(3), \square \otimes \Lambda_{1} \otimes \Lambda_{1}, V(1) \otimes V(2 n) \otimes V(3)\right)$ is a $P . V$. and there exists a relative invariant of degree 4 . However it is not a regular $P . V$.

$$
\left(G L(1) \times S p(n), \square \otimes A_{2}, V(1) \otimes V((n-1)(2 n+1))\right) \quad(n \geqq 3)
$$

Let $V^{\prime}=\left\{X \in M(2 n) \mid{ }^{t} X=-X\right\}$ and $\rho^{\prime}(g) X=s A X^{t} A$ for $g=(s, A) \epsilon$ $G L(1) \times S p(n) . \quad$ Then

$$
J=\left(\begin{array}{c|c}
0 & I_{n} \\
\hline-I_{n} & 0
\end{array}\right) \in V^{\prime}
$$


and its complement $V$ can be identified with $V((n-1)(2 n+1))$ (see Example 25 in $\S 1$ ). Then $\rho=\square \otimes \Lambda_{2}$ is the restriction of $\rho^{\prime}$ to $V$. Since ${ }^{t} A J A=J$ for $A \in S p(n)$, we have $(\rho(g) X) J=s A X^{t} A J=s A X J A^{-1}$ and hence eigen-values of $X J$ are invariant under the action of $g=(1, A) \in$ $G L(1) \times S p(n)$. Therefore if $n \geqq 3$, it cannot be a P.V. Note that the trace, i.e., the sum of eigen-values, of skew-symmetric matrices is zero.

Proposition 20. A triplet

$$
\left(G L(1) \times S p(n), \square \otimes A_{2}, V(1) \otimes V((n-1)(2 n+1))\right)
$$

is not a P.V. for $n \geqq 3$.

$$
\left(G L(1) \times S p(n) \times S p(m), \square \otimes \Lambda_{1} \otimes \Lambda_{1}, V(1) \otimes V(2 n) \otimes V(2 m)\right)
$$

$$
(n \geqq m \geqq 2)
$$

Let $V$ be the totality of $2 m \times 2 m$ skew-symmetric matrices, i.e., $V=$ $\left\{\left.X \in M(2 m)\right|^{t} X=-X\right\}$. Define the action $\rho_{\bar{H}}$ of $G=G L(1) \times S p(m)$ on $V$ by $\rho_{\bar{H}}(g) X=t A X^{t} A$ where $X \in V$ and $g=(t, A) \in G L(1) \times S p(m)$. Note that $\rho_{H_{-}}$is not irreducible. By Proposition 13 in $\S 2$, our triplet is a if and only if a triplet $\left(G, \rho_{\bar{H}}, V\right)$ is a P.V. As a representation space of $G, V$ decomposes to the direct sum $V=V(1) \oplus V((m-1)(2 m+1))$ where $V(1)$ is a one-dimensional vector space spanned by

$$
J=\left(\begin{array}{c|c}
0 & I_{m} \\
\hline-I_{m} & 0
\end{array}\right),
$$

and the action of $\rho$ on $V((m-1)(2 m+1))$ is equivalent to a triplet $\left(G L(1) \times S p(m), \square \otimes A_{2}, V(1) \otimes V((m-1)(2 m+1))\right)$ (See (17)). The projection of $V$ into $V((m-1)(2 m+1))$ is clearly surjective and G-equivariant. By Proposition 20, a triplet $\left(G L(1) \times S p(m), \square \otimes \Lambda_{2}, V(1) \otimes\right.$ $V((m-1)(2 m+1)))$ is not a P.V. for $m \geqq 3$, by Lemma 5 in $\S 2$. Assume that a triplet $\left(G, \rho_{E}, V\right)$ is a P.V. for $m=2$ and let $X_{0}=\left(x_{1}, x_{2}\right) \in V=$ $V(1) \oplus V(5)$ be its generic point. Then $x_{2}$ is a generic point of a triplet $\left(G L(1) \times S p(2), \square \otimes \Lambda_{2}, V(1) \otimes V(5)\right) \cong\left(G L(1) \times S O(5), \square \otimes \Lambda_{1}, V(1) \otimes V(5)\right)$ and the isotropy subgroup at $x_{2}$ is isomorphic to $S O(4) \times\{ \pm 1\}$ by Proposition 23. Since the action of $S O(4) \times\{ \pm 1\}$ on $x_{1}$ is given by $x_{1} \mapsto \pm x_{1}$, $X_{0}=\left(x_{1}, x_{2}\right)$ can not be generic point. This is a contradiction and hence 
a triplet $\left(G, \rho_{-}, V\right)$ is not a P.V. for $m \geqq 2$, i.e., our triplet is not a P.V.

Proposition 21. A triplet $\left(G L(1) \times S p(n) \times S p(m), \square \otimes \Lambda_{1} \otimes \Lambda_{1}, V(1)\right.$ $\otimes V(2 n) \otimes V(2 m))(n \geqq m \geqq 2)$ is not a P.V.

$$
\left(G L(1) \times S p(3), \square \otimes \Lambda_{3}, V(1) \otimes V(14)\right)
$$

First we consider $\left(G L(6), \Lambda_{3}, V(20)\right)$ (See (7)). If we restrict $\Lambda_{3}$ to the subgroup $G L(1) \times S p(3)$ of $G L(6), V(20)$ decomposes into a direct sum $V(20)=V(6) \oplus V(14)$ since the restriction of weights $\left\{\lambda_{i}+\lambda_{j}+\lambda_{k} \mid 1\right.$ $\leq i<j<k \leq 6\}$ of $G L(6)$ decomposes into $\left\{ \pm \lambda_{i} \mid i=1,2,3\right\} \cup\left\{ \pm \lambda_{1} \pm \lambda_{2} \pm \lambda_{3}\right.$, $\left.\pm \lambda_{1}, \pm \lambda_{2}, \pm \lambda_{3}\right\}$. The action $\rho$ of $G L(1) \times S p(3)$ on $V(14)$ is $\square \otimes \Lambda_{3}$. Note that the same notation $\Lambda_{3}$ is used for $G L(6)$ and $S p(3)$. Every element of $V(20)$ can be written uniquely in the form $x=\sum_{i<j<k} x_{i j k} u_{i} \wedge u_{j} \wedge u_{k}$ with $\left(x_{i j k}\right)$ forming an alternating tensor of rank three with coefficients in $C$. Moreover the element $x$ is contained in $V(14)$ if and only if $x_{i 14}$ $+x_{i 25}+x_{i 36}=0$ for $1 \leq i \leq 6$. Let $g$ be the Lie algebra of $G L(1) \times S p(3)$.

$$
\mathfrak{g}=\left\{A=\left\{\begin{array}{ccc|ccc}
d+a_{1} & a_{12} & a_{13} & b_{1} & b_{12} & b_{13} \\
a_{21} & d+a_{2} & a_{23} & b_{12} & b_{2} & b_{23} \\
a_{31} & a_{32} & d+a_{3} & b_{13} & b_{23} & b_{3} \\
\hline c_{1} & c_{12} & c_{13} & d-a_{1} & -a_{21} & -a_{31} \\
c_{12} & c_{2} & c_{23} & -a_{12} & d-a_{2} & -a_{32} \\
c_{13} & c_{23} & c_{3} & -a_{13} & -a_{23} & d-a_{3}
\end{array}\right\} \in \operatorname{gr}(6)\right\}
$$

We shall calculate the isotropy subalgebra $g_{X_{0}}$ at $X_{0}=u_{1} \wedge u_{2} \wedge u_{3}$ $+u_{4} \wedge u_{5} \wedge u_{6}$. Since $d \rho(A) X_{0}=\left(3 d+a_{1}+a_{2}+a_{3}\right) u_{1} \wedge u_{2} \wedge u_{3}+(3 d-$ $\left.a_{1}-a_{2}-a_{3}\right) u_{4} \wedge u_{5} \wedge u_{6}+b_{1} u_{1} \wedge u_{5} \wedge u_{6}+b_{2} u_{4} \wedge u_{2} \wedge u_{6}+b_{3} u_{4} \wedge u_{5} \wedge u_{3}$ $+c_{1} u_{4} \wedge u_{2} \wedge u_{3}+c_{2} u_{1} \wedge u_{5} \wedge u_{3} \wedge c_{3} u_{1} \wedge u_{2} \wedge u_{6}+\left(c_{12} u_{3}-b_{12} u_{6}\right) \wedge\left(u_{1} \wedge\right.$ $\left.u_{4}-u_{2} \wedge u_{5}\right)+\left(c_{23} u_{1}-b_{23} u_{4}\right) \wedge\left(u_{2} \wedge u_{5}-u_{3} \wedge u_{6}\right)+\left(c_{13} u_{2}-b_{13} u_{5}\right) \wedge\left(u_{3} \wedge\right.$ $\left.u_{6}-u_{1} \wedge u_{4}\right)$, we have

$$
\begin{aligned}
\mathfrak{g}_{X_{0}} & =\left\{A \in \mathfrak{g} \mid d \rho(A) X_{0}=0\right\} \\
& =\left\{A \in \mathfrak{g} \mid d=a_{1}+a_{2}+a_{3}=0,\left(b_{i j}\right)=\left(c_{i j}\right)=0\right\} \\
& =\left\{\left(\frac{\Lambda}{0} \mid \frac{0}{-{ }^{t} \Lambda}\right) \mid \Lambda \in \mathfrak{g}((3)\} \cong \mathfrak{I}(3) .\right.
\end{aligned}
$$

Since $\operatorname{dim} \mathfrak{g}_{x_{0}}=8=\operatorname{dim} G L(1) \times S p(3)-\operatorname{dim} V(14)$, it is a regular P.V. by Proposition 25 in $\S 4$. Similarly, for $X_{0}^{\prime}=u_{1} \wedge u_{2} \wedge u_{6}+u_{3} \wedge\left(u_{1} \wedge\right.$ 
$\left.u_{4}-u_{2} \wedge u_{5}\right)$, we have $d \rho(A) X_{0}^{\prime}=\left\{\left(3 d+a_{3}\right) u_{3}+c_{3} u_{6}\right\} \wedge\left(u_{1} \wedge u_{4}-u_{2} \wedge u_{5}\right)$ $-\left\{\left(a_{13}+a_{32}\right) u_{1}+c_{13} u_{4}\right\} \wedge\left(u_{2} \wedge u_{5}-u_{3} \wedge u_{6}\right)-\left\{\left(a_{23}+a_{31}\right) u_{2}+c_{23} u_{5}\right\} \wedge\left(u_{3} \wedge\right.$ $\left.u_{6}-u_{1} \wedge u_{4}\right)+\left(c_{2} u_{6}-2 a_{12} u_{3}\right) \wedge u_{1} \wedge u_{5}-\left(c_{1} u_{6}+2 a_{21} u_{3}\right) \wedge u_{2} \wedge u_{4}-2 c_{12} u_{3} \wedge$ $u_{4} \wedge u_{5}+\left(b_{3}+2 b_{12}\right) u_{1} \wedge u_{2} \wedge u_{3}+\left(3 d+a_{1}+a_{2}-a_{3}\right) u_{1} \wedge u_{2} \wedge u_{6}$ and hence $\mathfrak{g}_{X_{0}^{\prime}}=\left\{A \in \mathfrak{g} \mid d \rho(A) X_{0}^{\prime}=0\right\}$ is given by

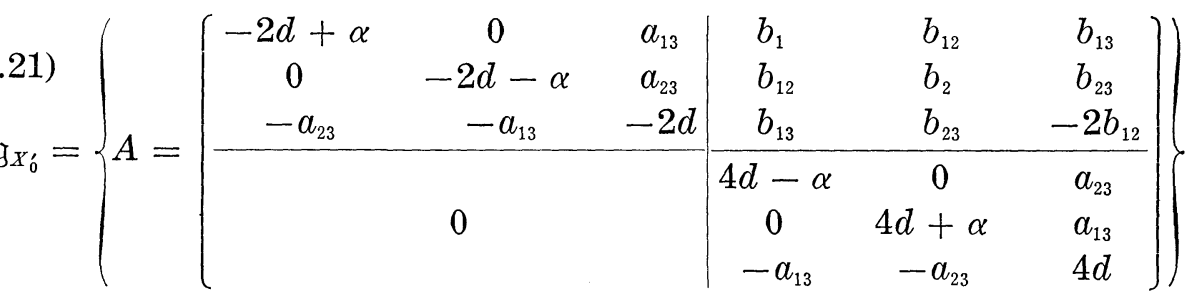

$$
\cong\left(\mathfrak{g l}(1) \oplus \mathfrak{g}_{\mathfrak{D}}(3)\right) \oplus \mathfrak{u}(5)
$$

where $\mathfrak{u}(5)$ is the Lie algebra of a 5-dimensional vector group. Since $\operatorname{dim}\left\{d \rho(A) X_{0}^{\prime} \mid A \in \mathfrak{g}\right\}=13$, the orbit of $X_{0}^{\prime}$ is of codimension 1. For $A \in \mathfrak{g}_{X_{0}^{\prime}}$, the trace $\operatorname{tr}_{V} A$ in $V=V(14)$ is $14 \times 3 d=42 d$ and the trace $\operatorname{tr} \operatorname{ad} g_{x_{0}^{\prime}} A$ of the adjoint representation is $-30 d$, and hence we have

$$
\operatorname{deg} f=\frac{\operatorname{tr}_{V} A+\operatorname{tr} \operatorname{ad} A}{\operatorname{tr}_{V} A} \cdot \operatorname{dim} V=\frac{42 d-30 d}{42 d} \times 14=4
$$

by Proposition 15 in $\S 4$, where $f(x)$ is an irreducible relative invariant polynomial. This shows that the restriction of the relative invariant of degree 4 of $\left(G L(6), \Lambda_{3}, V(20)\right)$ is still irreducible, and it is the relative invariant of $\left(G L(1) \times S p(3), \square \otimes \Lambda_{3}, V(1) \otimes V(14)\right)$.

Put

$$
X=\left(\begin{array}{lll}
x_{423} & x_{143} & x_{124} \\
x_{523} & x_{153} & x_{125} \\
x_{623} & x_{163} & x_{126}
\end{array}\right) \text { and } Y=\left(\begin{array}{lll}
x_{156} & x_{416} & x_{451} \\
x_{256} & x_{426} & x_{452} \\
x_{356} & x_{436} & x_{453}
\end{array}\right)
$$

Since $x_{i 14}+x_{i 25}+x_{i 36}=0, X$ and $Y$ are symmetric matrices. We denote by $X_{i j}$ the matrix obtained from $X$ by crossing out its $i$-th line and $j$-th column. Put $x_{0}=x_{123}$ and $y_{0}=x_{456}$. Then the relative invariant $f(x)$ for $x=\sum x_{i j k} u_{i} \wedge u_{j} \wedge u_{k} \in V(14)$ is given by $f(x)=\left(x_{0} y_{0}-\operatorname{tr} X Y\right)^{2}+$ $4 x_{0} \operatorname{det} Y+4 y_{0} \operatorname{det} X-4 \sum_{i, j} \operatorname{det}\left(X_{i j}\right) \cdot \operatorname{det}\left(Y_{j i}\right)$ (See (7)). This space was investigated in detail by J. Igusa (See [2]).

Proposition 22. A triplet $\left(G L(1) \times S p(3), \square \otimes \Lambda_{3}, V(1) \otimes V(14)\right)$ is a regular $P . V$. and its generic isotropy subgroup is locally isomorphic to 
SL(3). The relative invariant is of degree 4 .

$$
\cdot\left(S O(n) \times G L(m), \Lambda_{1} \otimes \Lambda_{1}, V(n) \otimes V(m)\right) \quad\left(n \geqq 3, \frac{n}{2} \geqq m \geqq 1\right) .
$$

The special orthogonal group $S O(n)$ is defined by

$$
S O(n)=\left\{g \in S L(n) \mid{ }^{t} g K g=K\right\}
$$

for some symmetric $n \times n$ non-singular matrix $K$. Since we consider them over the algebraically closed field $C$, we may assume that $K=I_{n}$, i.e., the identity matrix of size $n$. We identify $V=V(n) \otimes V(m)$ with $n \times m$ matrices $M(n, m)$. Then the action $\rho=\Lambda_{1} \otimes \Lambda_{1}$ is given by $\rho(g) X$ $=g_{1} X^{t} g_{2}$ for $g=\left(g_{1}, g_{2}\right) \in S O(n) \times G L(m)$. We shall calculate the isotropy subgroup $G_{X_{0}}$ at $X_{0}={ }^{t}\left(I_{m} O\right) \in M(n, m)$. An element of $S O(n)$ can be written as follows.

$$
A=\left(\begin{array}{ll}
A_{1} & A_{2} \\
A_{3} & A_{4}
\end{array}\right) \text { where } A_{1} \in M(m), A_{2},{ }^{t} A_{3} \in M(m, n-m),
$$

$A_{4} \in M(n-m)$, and ${ }^{t} A A=1_{n}$, $\operatorname{det} A=1$. Then for $g=(A, B) \in S O(n)$ $\times G L(m)$, we have

$$
\rho(g) X_{0}=\left(\begin{array}{cc}
A_{1} & A_{2} \\
A_{3} & A_{4}
\end{array}\right)\left(\begin{array}{c}
I_{m} \\
0
\end{array}\right)^{t} B=\left(\begin{array}{c}
A_{1}{ }^{t} B \\
A_{3}{ }^{t} B
\end{array}\right)
$$

and hence the isotropy subgroup $G_{X_{0}}$ is given by $G_{X_{0}}=\{(A, B) \in S O(n) \times$ $\left.G L(m) \mid A_{1}{ }^{t} B=I_{n}, A_{3}{ }^{t} B=0\right\}$. The equation $A_{1}{ }^{t} B=I_{n}, A_{3}{ }^{t} B=0$ implies that $A_{3}=\left(A_{3}{ }^{t} B\right)^{t} B^{-1}=0$ and $B={ }^{t} A_{1}^{-1}$. Since $\left(\begin{array}{cc}A_{1} & A_{2} \\ 0 & A_{4}\end{array}\right)$ is in $S O(n)$ if and only if $\operatorname{det} A_{1} \cdot \operatorname{det} A_{4}=1,{ }^{t} A_{1} A_{1}=I_{m},{ }^{t} A_{1} A_{2}=0$ (i.e. $A_{2}=0$ ) and ${ }^{t} A_{4} A_{4}=I_{n-m}$, we have

$$
\begin{aligned}
G_{X_{0}} & =\left\{\left(\left(\frac{A_{1}}{0} \mid \frac{0}{A_{4}}\right),\left({ }^{t} A_{1}^{-1}\right)\right) \mid A_{1} \in O(m), A_{4} \in O(n-m), \operatorname{det} A_{1} \cdot \operatorname{det} A_{4}=1\right\} \\
& \cong S O(m) \times S O(n-m) \times\{ \pm 1\} .
\end{aligned}
$$

Since $\operatorname{dim} G_{x_{0}}=\frac{1}{2} m(m+1)+\frac{1}{2}(n-m)(n-m+1)=\frac{1}{2} n(n+1)+m^{2}$ $-n m=\operatorname{dim} S O(n) \times G L(m)-\operatorname{dim} V$, it is a regular P.V. by Proposition 25 in $\S 4$. The relative invariant $f(X)$ is given by $f(X)=\operatorname{det}\left({ }^{t} X X\right)$. In general, $f(X)=\operatorname{det}\left({ }^{t} X K X\right)$ if $S O(n)$ is given by $\left\{\left.g \in S L(n)\right|^{t} g K g=K\right\}$ since $f\left(A X^{t} B\right)=\operatorname{det}\left(B^{t} X^{t} A K A X^{t} B\right)=\operatorname{det}\left(B^{t} X K X^{t} B\right)=(\operatorname{det} B)^{2} \cdot f(X)$ for 
$(A, B) \in S O(n) \times G L(m)$. Any rational character $\chi$ of $S O(n) \times G L(m)$ is of the form $\chi(g)=(\operatorname{det} B)^{\ell}$ for some $\ell \in Z$ where $g=(A, B) \in S O(n) \times$ $G L(m)$. Since $\left.\chi\right|_{a_{X_{0}}} \equiv 1$ if and only if $\ell$ is even, any relative invariant is of the form $c \cdot f(X)^{r}\left(r \in Z, c \in C^{\times}\right)$by Proposition 19 in $\S 4$ and hence $f(X)=\operatorname{det}\left({ }^{t} X K X\right)$ is irreducible.

Proposition 23. A triplet $\left(S O(n) \times G L(m), \Lambda_{1} \otimes \Lambda_{1}, V(n) \otimes V(m)\right)$ with $n \geqq 3, \frac{n}{2} \geqq m \geqq 1$, is a regular P.V. and its generic isotropy subgroup is isomorphic to $S O(m) \times S O(n-m) \times\{ \pm 1\}$. The relative invariant is of degree $2 \mathrm{~m}$.

Note that Proposition 23 holds even if $n \geqq m>\frac{n}{2}$ although in this case, it is not reduced.

$$
\left(G L(1) \times S O(n) \times S p(m), \square \otimes \Lambda_{1} \otimes \Lambda_{1}, V(1) \otimes V(n) \otimes V(2 m)\right)
$$

$$
(n>2 m \geqq 4)
$$

By Proposition 14 in $\S 2$, it is a P.V. if and only if a triplet $\left(G L(1) \times S p(m), \square \otimes 2 \Lambda_{1}, V(1) \otimes V(m(2 m+1))\right)$ is a P.V. Since $2 \Lambda_{1}$ is the adjoint representation of $S p(m)$ and $\operatorname{rank} S p(m) \geqq 2(m \geqq 2)$, it is not a P.V. by Proposition 2.

Proposition 24. A triplet $\left(G L(1) \times S O(n) \times S p(m), \square \otimes \Lambda_{1} \otimes \Lambda_{1}, V(1)\right.$ $\otimes V(n) \otimes V(2 m))$ is not a P.V.

A short outline of the theory of the spin representation necessary for the following exposition will be presented below.

Let $V$ be a vector space over the complex number field $C$ of even dimension $n=2 m$. Let $Q$ be a non-degenerate quadratic form on $V$, and let $B(x, y)$ be the associated bilinear form, i.e., $B(x, y)=Q(x+y)$ $-Q(x)-Q(y)$ for $x, y \in V$.

Then there exists a basis $\left\{e_{1}, \cdots, e_{m}, f_{1}, \cdots, f_{m}\right\}$ of $V$ satisfying $B\left(e_{i}, e_{j}\right)=B\left(f_{i}, f_{j}\right)=0, B\left(e_{i}, f_{j}\right)=\delta_{i j}$ and we have

$$
\mathbb{Q}\left(\sum_{i=1}^{m} x_{i} e_{i}+\sum_{i=1}^{m} y_{i} f_{i}\right)=\sum_{i=1}^{m} x_{i} y_{i}
$$

for any $x_{i}, y_{i} \in C$. Let $B_{0}$ be the bilinear form on $V \times V$ defined by

$$
B_{0}\left(\sum x_{i} e_{i}+\sum y_{i} f_{i}, \sum x_{i}^{\prime} e_{i}+\sum y_{i}^{\prime} f_{i}\right)=\sum x_{i}^{\prime} y_{i} .
$$


Note that $B_{0}(x, x)=Q(x)$ for $x \in V$.

Let $T(V)=\sum_{k=0}^{\infty} V \overbrace{\otimes \cdots \otimes}^{k} V$ be the tensor algebra over $V$, and let $I_{Q}$ be the two-sided ideal of $T(V)$ generated by the subset $\{x \otimes x-Q(x)$. $1 \mid x \in V\}$. Then the quotient algebra $C(Q)=T(V) / I_{Q}$ is called the Clifford algebra. Put $T^{+}(V)=\sum_{k=0}^{\infty} V \overbrace{\otimes \cdots \otimes}^{2 k} V$ and let $\varphi: T(V) \rightarrow C(Q)$ be the canonical map from $T(V)$ onto $C(Q)$. The image $\varphi\left(T^{+}(V)\right)$ of $T^{+}(V)$ is called the even Clifford algebra and denoted by $C^{+}(Q)$. It is known that $C(Q) \cong M\left(2^{m}, C\right)$ and $C^{+}(Q) \cong M\left(2^{m-1}, C\right) \oplus M\left(2^{m-1}, C\right)$. The orthogonal group $O(Q, V) \mathrm{w}, \mathrm{r}, \mathrm{t}, Q$ and the special orthogonal group $S O(Q, V) \mathrm{w}, \mathrm{r}, \mathrm{t}, Q$ are defined as follows.

$$
\begin{aligned}
O(Q, V) & =\{g \in G L(V) \mid Q(g x)=Q(x) \text { for any } x \in V\} \\
S O(Q, V) & =O(Q, V) \cap S L(V)
\end{aligned}
$$

We shall also define the Clifford group $\Gamma(Q)$ and the even Clifford group $\Gamma^{+}(Q)$ as follows.

$$
\begin{aligned}
\Gamma(Q) & =\left\{\left.s \in C(Q)\right|^{\exists} s^{-1}, s V s^{-1} \subset V\right\} \\
\Gamma^{+}(Q) & =\Gamma(Q) \cap C^{+}(Q)
\end{aligned}
$$

Let $\chi$ be the representation of $\Gamma(Q)$ on $V$ defined by $\chi(s) v=s v s^{-1}$ for $s \in \Gamma(Q)$ and $x \in V$. Since $Q(\chi(s) v)=\left(s v s^{-1}\right)^{2}=s v^{2} s^{-1}=s Q(v) s^{-1}=Q(v)$, we have $\chi(s) \in O(Q, V)$. This $\chi$ is called the vector representation of $\Gamma(Q)$.

Let $\alpha$ be the anti-automorphism of $T(V)$ defined by $\alpha\left(v_{1} \otimes \cdots \otimes v_{k}\right)$ $=v_{k} \otimes \cdots \otimes v_{1} . \quad$ As $\alpha\left(I_{Q}\right) \subset I_{Q}, \alpha$ induces the anti-automorphism on $C(Q)$, which is also denoted by $\alpha$. Note that $\alpha$ fixes an element of $V$ and hence $s v s^{-1}=\alpha\left(s v s^{-1}\right)=\alpha(s)^{-1} \alpha(v) \alpha(s)=\alpha(s)^{-1} v \alpha(s)$ for $s \in \Gamma(Q), v \in V$. This implies that $\alpha(s) s v=v \alpha(s) s$ and hence $\alpha(s) s$ is an element of the center $\boldsymbol{C}$ of $C(Q)$. Since $s$ is invertible, we have $\alpha(s) s \in C^{\times}$. We shall define the spin group $\operatorname{Spin}(Q)$ as $\operatorname{Spin}(Q)=\left\{s \in \Gamma^{+}(Q) \mid \alpha(s) s=1\right\}$. It is connected, simply connected and semisimple. Moreover if $n \neq 4$, then it is simple. It is well-known that the following exact sequence (5.27) holds.

$$
1 \longrightarrow\{ \pm 1\} \longrightarrow \operatorname{Spin}(Q) \stackrel{\chi}{\longrightarrow} \operatorname{SO}(Q, V) \longrightarrow 1 \quad \text { (exact) . }
$$

Now we shall construct the half-spin representation of the spin group $\operatorname{Spin}(Q)$. Let $\Lambda(V)=\sum_{k=0}^{n} \Lambda^{k}(V)$ be the exterior algebra of $V$. For each $x \in V$, let $\rho(x)$ be an element of $\operatorname{End}_{c}(\Lambda(V))$ defined by $\rho(x) \lambda=\left(L_{x}+\right.$ 
$\left.\delta_{x}\right) \lambda(\lambda \in \Lambda(V))$ where $L_{x} \lambda=x \wedge \lambda$ and

$$
\delta_{x}\left(v_{1} \wedge \cdots \wedge v_{k}\right)=\sum_{i=1}^{k}(-1)^{i-1} B_{0}\left(x, v_{i}\right) v_{1} \wedge \cdots \wedge v_{i-1} \wedge v_{i+1} \wedge \cdots \wedge v_{k}
$$

One can easily check that $\rho(x)^{2}=Q(x) \cdot 1$, and hence the representation of $V$ on $\Lambda(V)$ can be extended to that of the Clifford algebra $C(Q)$ on $\Lambda(V)$. Put $f=f_{1} \wedge \cdots \wedge f_{m}$ and $M=\Lambda(V) \wedge f$. Then one can check that $M$ is a $\rho$-invariant subspace of $\Lambda(V)$.

Let $E$ be the subspace of $V$ generated by $\left\{e_{1}, \cdots, e_{m}\right\}$. Then the map $\varphi: \Lambda(E) \rightarrow M$ defined by $\varphi(\mu)=\mu \wedge f$ for $\mu \in \Lambda(E)$, is clearly a linear isomorphism. We identify $\Lambda(E)$ with $M$ by this map, and hence we obtain the representation $\rho$ of $C(Q)$ on $\Lambda(E)$. The subspaces $\Lambda^{+}(E)=$ $\sum_{k \text { : even }} \Lambda^{k}(E)$ and $\Lambda^{-}(E)=\sum_{k \text { : odd }} \Lambda^{k}(E)$ of $\Lambda(E)$ are the irreducible representation spaces with respect to the restriction of $\rho$ to the spin group $\operatorname{Spin}(Q)$. This representation of $\operatorname{Spin}(Q)$ on $\Lambda^{+}(E)$ (resp. $\Lambda^{-}(E)$ ) is called the even (resp. odd) half-spin representation.

These two half-spin representations are inequivalent, however, they are transformed to each other by the outer automorphism of Spin (Q). Therefore we shall consider only the even half-spin representation of $\operatorname{Spin}(Q)$

Now we shall calculate the infinitesimal representation $d \rho$ of the half-spin representation.

Let $E_{i j}$ be the matrix unit of degree $m(1 \leq i, j \leq m)$ and put $E_{i j}^{\prime}$ $=E_{i j}-E_{j i}$. Then an element $A$ of the Lie algebra $\mathfrak{g}=\mathfrak{o}(2 m, C)$ of the spin group Spin $(Q)$ can be written as follows (See $\S 1$ ).

$$
\begin{aligned}
A= & \sum_{i \neq j} a_{i j}\left(\begin{array}{cc}
E_{i j} & 0 \\
0 & -E_{j i}
\end{array}\right)+\sum_{i<j} b_{i j}\left(\begin{array}{cc}
0 & E_{i j}^{\prime} \\
0 & 0
\end{array}\right) \\
& +\sum_{i<j} c_{i j}\left(\begin{array}{cc}
0 & 0 \\
E_{i j}^{\prime} & 0
\end{array}\right)+\sum_{i=1}^{m} a_{i}\left(\begin{array}{cc}
E_{i i} & 0 \\
0 & -E_{i i}
\end{array}\right) .
\end{aligned}
$$

By the definition the product in $C(Q)$ is given by $e_{i} f_{i}+f_{i} e_{i}=1, e_{i}^{2}=f_{i}^{2}$ $=0, e_{i} f_{j}=-f_{j} e_{i}, e_{i} e_{j}=-e_{j} e_{i}$ and $f_{i} f_{j}=-f_{j} f_{i}(j \neq i, i, j=1, \cdots, m)$. We shall consider an element $s=1+t e_{i} f_{j}(t \in C, i \neq j)$ of $C(Q)$. Then $\alpha(s)=1+t f_{j} e_{i}$ and hence $\alpha(s) s=1$ i.e. $s^{-1}=\alpha(s)$. Since $\chi(s) e_{k}=s e_{k} s^{-1}$ $=\left(1+t e_{i} f_{j}\right) e_{k}\left(1+t f_{j} e_{i}\right)$, we have $\chi(s) e_{k}=e_{k}$ for any $k \neq j$ and $\chi(s) e_{j}$ $=e_{j}+t e_{i}$. Also we have $\chi(s) f_{k}=f_{k}$ for any $k \neq i$ and $\chi(s) f_{i}=f_{i}-t f_{j}$. This implies that $s=1+t e_{i} f_{j} \in \operatorname{Spin}(Q)$ and 


$$
\chi\left(1+t e_{i} f_{j}\right)=\exp t\left(\begin{array}{cc}
E_{i j} & 0 \\
0 & -E_{j i}
\end{array}\right) \in S O(Q, V) .
$$

Similarly we have the following relations.

1) $\chi\left(1+t e_{i} f_{j}\right)=\exp t\left(\begin{array}{cc}E_{i j} & 0 \\ 0 & -E_{j i}\end{array}\right) \in S O(Q, V)(i \neq j)$.
2) $\chi\left(1+t e_{i} e_{j}\right)=\exp t\left(\begin{array}{cc}0 & E_{i j}^{\prime} \\ 0 & 0\end{array}\right) \in S O(Q, V)(i<j)$.
3) $\chi\left(1+t f_{i} f_{j}\right)=\exp t\left(\begin{array}{cc}0 & 0 \\ E_{i j}^{\prime} & 0\end{array}\right) \in S O(Q, V)(i<j)$.

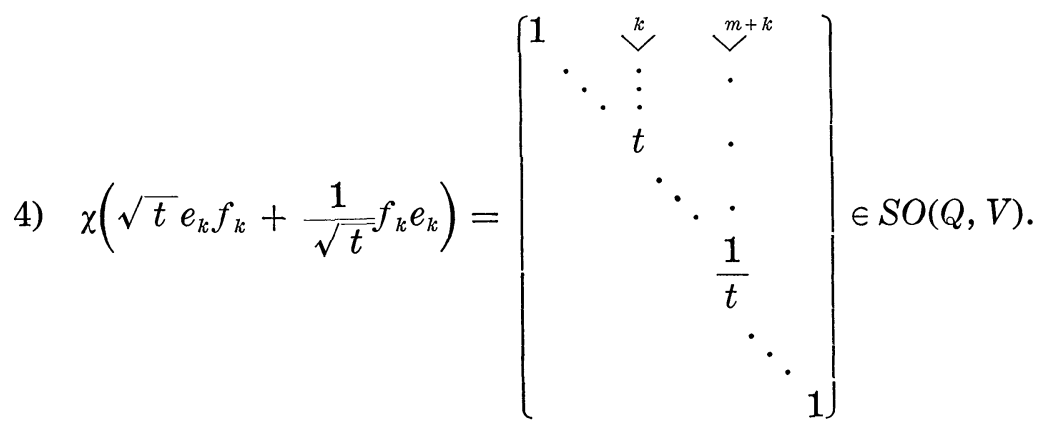

Since $\chi$ is an isomorphism in a neighborhood of the identity, we have

$$
\begin{aligned}
d \rho\left(\begin{array}{cc}
E_{i j} & 0 \\
0 & -E_{j i}
\end{array}\right) \lambda= & \lim _{t \rightarrow 0} \frac{1}{t}\left(\rho\left(\exp t\left(\begin{array}{cc}
E_{i j} & 0 \\
0 & -E_{j i}
\end{array}\right)\right)-\rho(1)\right) \lambda \\
= & \lim _{t \rightarrow 0} \frac{1}{t}\left(\rho\left(1+t e_{i} f_{j}\right)-\rho(1)\right) \lambda=\rho\left(e_{i}\right) \rho\left(f_{j}\right) \lambda=e_{i} \delta_{f_{j}} \lambda \\
& \text { for } i \neq j, \lambda \in \Lambda^{+}(E) .
\end{aligned}
$$

Similarly we have
$\left.1^{\prime}\right) \quad d \rho\left(\begin{array}{cc}E_{i j} & 0 \\ 0 & -E_{j i}\end{array}\right) \lambda=\rho\left(e_{i}\right) \rho\left(f_{j}\right) \lambda=e_{i} \delta_{f_{j}} \lambda$
$\left.2^{\prime}\right) \quad d \rho\left(\begin{array}{cc}0 & E_{i j}^{\prime} \\ 0 & 0\end{array}\right) \lambda=\rho\left(e_{i}\right) \rho\left(e_{j}\right) \lambda=e_{i} \wedge e_{j} \wedge \lambda$
$\left.3^{\prime}\right) \quad d \rho\left(\begin{array}{cc}0 & 0 \\ E_{i j}^{\prime} & 0\end{array}\right) \lambda=\rho\left(f_{i}\right) \rho\left(f_{j}\right) \lambda=\delta_{f_{i}} \delta_{f_{j}} \lambda$
$\left.4^{\prime}\right) \quad d \rho\left(\begin{array}{cc}\stackrel{k}{1} & { }^{m+k} \\ & -1\end{array}\right) \lambda=\rho\left(e_{k}\right) \rho\left(f_{k}\right) \lambda-\frac{1}{2} \lambda$

and hence for an element $A$ in (5.28) of $\mathfrak{g n}(2 \mathrm{~m})$, we have the following (5.29). 


$$
d \rho(A) \lambda=\sum_{i \neq j} a_{i j} e_{i} \delta_{f_{j}} \lambda+\sum_{i=1}^{m} a_{i}\left(e_{i} \delta_{f_{i}}-\frac{1}{2}\right) \lambda+\sum_{i<j} b_{i j} e_{i} e_{j} \lambda+\sum_{i<j} c_{i j} \delta_{f_{i}} \delta_{f_{j}} \lambda
$$

Next we shall consider the case when the dimension $n$ of $V$ is odd, i.e., $n=2 m+1$. Let $Q$ be a nondegenerate quadratic form on $V$, and let $B(x, y)$ be the associated bilinear form. Let $V_{0}$ denote the subspace of $V$ generated by $e_{1}, \cdots, e_{m}, f_{1}, \cdots, f_{m}$ satisfying $B\left(e_{i}, e_{j}\right)=B\left(f_{i}, f_{j}\right)=0$ and $B\left(e_{i}, f_{j}\right)=\delta_{i j}$. Then the subspace of $V$ orthogonal to $V_{0}$ is of the form $C v_{0}$ for some $v_{0}$ in $V$ satisfying $Q\left(v_{0}\right) \neq 0$. We may assume that $Q\left(v_{0}\right)=1$. Now consider a vector space $V_{1}$ of dimension $n+1$ and represent it as $V_{1}=V+C v_{1}$. Let $Q_{1}$ be the quadratic form on $V_{1}$ defined by $Q_{1}\left(V+\lambda v_{1}\right)=Q(v)-\lambda^{2}$ for $v \in V, \lambda \in C$, and let $B_{1}(x, y)$ be the associated bilinear form of $Q_{1}$. Then, if we put $e_{m+1}=\frac{1}{2}\left(v_{0}+v_{1}\right)$ and $f_{m+1}=\frac{1}{2}\left(v_{0}-v_{1}\right)$, we have $B_{1}\left(e_{i}, e_{j}\right)=B_{1}\left(f_{i}, f_{j}\right)=0$ and $B_{1}\left(e_{i}, f_{j}\right)=\delta_{i j}$ for $1 \leq i, j \leq m+1$. Let $\operatorname{Spin}\left(Q_{1}\right)$ be the spin group of $\left(V_{1}, Q_{1}\right)$, and let $\chi_{1}$ be its vector representation. Then the spin group $\operatorname{Spin}(Q)$ is defined by $\operatorname{Spin}(Q)=\left\{s \in \operatorname{Spin}\left(Q_{1}\right) \mid \chi_{1}(s) v_{1}=v_{1}\right\}$. By restricting the half-spin representation of $\operatorname{Spin}\left(Q_{1}\right)$ to $\operatorname{Spin}(Q)$, we obtain the spin representation of $\operatorname{Spin}(Q)$ (See [2], [3]).

In the following, we denote the spin group by $\operatorname{Spin}(n)$ instead of $\operatorname{Spin}(Q)$, and denote the element $e_{i_{1}} \wedge \cdots \wedge e_{i_{k}}$ of $\Lambda^{+}(E)$ by $e_{i_{1}} \cdots e_{i_{k}}$.

$$
\left(\operatorname{Spin}(7) \times G L(d), \text { spin rep. } \otimes \Lambda_{1}, V(8) \otimes V(d)\right) \quad(1 \leq d \leq 4)
$$

First of all, we shall calculate the half-spin representation $d \rho_{1}$ of $\mathfrak{D}(8, C)\left(=D_{4}\right)$. The representation space $V(8)$ is spanned by $1, e_{i} e_{j}, e_{1} e_{2} e_{3} e_{4}$ $(1 \leq i<j \leq 4)$. We may assume that an element $A$ of $\mathfrak{p}(8, C)$ is of the form (5.28). Then by (5.29) we can calculate $d \rho_{1}$. For example $d \rho_{1}(A) e_{1} e_{2}=\sum_{i \neq 1} a_{i 1} e_{i} e_{2}-\sum_{i \neq 2} a_{i 2} e_{i} e_{1}+\frac{1}{2}\left(a_{1}+a_{2}-a_{3}-a_{4}\right) e_{1} e_{2}+b_{34} e_{3} e_{4} e_{1} e_{2}$ $+c_{12} \delta_{f_{1}} \delta_{f_{2}} e_{1} e_{2}=-a_{31} e_{2} e_{3}-a_{41} e_{2} e_{4}+a_{32} e_{1} e_{3}+a_{42} e_{1} e_{4}+\frac{1}{2}\left(a_{1}+a_{2}-a_{3}-a_{4}\right) e_{1} e_{2}$ $+b_{34} e_{1} e_{2} e_{3} e_{4}-c_{12}$. Hence we have

$$
d \rho_{1}(A) x=\left(\begin{array}{r|r|r|r|r|r|r|r}
A_{1} & -c_{12} & -c_{13} & -c_{14} & 0 & c_{34} & -c_{24} & c_{23} \\
b_{12} & A_{2} & a_{23} & a_{24} & -c_{34} & 0 & -a_{14} & a_{13} \\
b_{13} & a_{32} & A_{3} & a_{34} & c_{24} & a_{14} & 0 & -a_{12} \\
b_{14} & a_{42} & a_{43} & A_{4} & -c_{23} & -a_{13} & a_{12} & 0 \\
\hline 0 & b_{34} & -b_{24} & b_{23} & -A_{1} & -b_{12} & -b_{13} & -b_{14} \\
-b_{34} & 0 & a_{41} & -a_{31} & c_{12} & -A_{2} & -a_{32} & -a_{42} \\
b_{24} & -a_{41} & 0 & a_{21} & c_{13} & -a_{23} & -A_{3} & -a_{43} \\
-b_{23} & a_{31} & -a_{21} & 0 & c_{14} & -a_{24} & -a_{34} & -A_{4}
\end{array}\right]\left(\begin{array}{c}
x_{1} \\
x_{2} \\
x_{3} \\
x_{4} \\
x_{5} \\
x_{6} \\
x_{7} \\
x_{8}
\end{array}\right)
$$


where $x=x_{1}+x_{2} e_{1} e_{2}+x_{3} e_{1} e_{3}+x_{4} e_{1} e_{4}+x_{5} e_{1} e_{2} e_{3} e_{4}-x_{6} e_{3} e_{4}+x_{7} e_{2} e_{4}-x_{8} e_{2} e_{3}$,

$$
\begin{array}{ll}
A_{1}=-\frac{a_{1}+a_{2}+a_{3}+a_{4}}{2}, & A_{2}=\frac{a_{1}+a_{2}-a_{3}-a_{4}}{2}, \\
A_{3}=\frac{a_{1}-a_{2}+a_{3}-a_{4}}{2}, & A_{4}=\frac{a_{1}-a_{2}-a_{3}+a_{4}}{2} .
\end{array}
$$

Since $d \rho_{1}(A)$ in (5.30) is the same form as in (5.28), $d \rho_{1}(A) \in \mathfrak{p}(8, C)$ and it leaves the quadratic form $q(x)=x_{1} x_{5}+x_{2} x_{6}+x_{3} x_{7}+x_{4} x_{8}$ invariant (See Example 28 in $\S 1$ ).

Put $v_{1}={ }^{t}(0001000-1) \in C^{8}$. Then the Lie algebra $\mathfrak{o}(7, C)$ of $\operatorname{Spin}(7)$ is given by $\mathfrak{o}(7, C)=\left\{A \in \mathfrak{o}(8, C) \mid A v_{1}=0\right\}$ and hence an element $A$ of $\mathfrak{v}(8, C)$ of the form $(5.28)$ is in $\mathfrak{o}(7, C)$ if and only if

$$
A v_{1}={ }^{t}\left(a_{14}-b_{14}, a_{24}-b_{24}, a_{34}-b_{34}, a_{4}, c_{14}+a_{41}, c_{24}+a_{42}, c_{34}+a_{43}, a_{4}\right)=0 \text {. }
$$

Thus the spin representation $d \rho_{1}$ of $\mathfrak{o}(7, C)$ is given as follows.

$$
d \rho_{1}(A) x=\left(\begin{array}{r|r|r|r|r|r|r|r}
A_{1}^{\prime} & -c_{12} & -c_{13} & -c_{14} & 0 & c_{34} & -c_{24} & c_{23} \\
b_{12} & A_{2}^{\prime} & a_{23} & b_{24} & -c_{34} & 0 & -b_{14} & a_{13} \\
b_{13} & a_{32} & A_{3}^{\prime} & b_{34} & c_{24} & b_{14} & 0 & -a_{12} \\
b_{14} & -c_{24} & -c_{34} & A_{4}^{\prime} & -c_{23} & -a_{13} & a_{12} & 0 \\
\hline 0 & b_{34} & -b_{24} & b_{23} & -A_{1}^{\prime} & -b_{12} & -b_{13} & -b_{14} \\
-b_{34} & 0 & -c_{14} & -a_{31} & c_{12} & -A_{2}^{\prime} & -a_{32} & c_{24} \\
b_{24} & c_{14} & 0 & a_{21} & c_{13} & -a_{23} & -A_{3}^{\prime} & c_{34} \\
-b_{23} & a_{31} & -a_{21} & 0 & c_{14} & -b_{24} & -b_{34} & -A_{4}^{\prime}
\end{array}\right]\left(\begin{array}{c}
x_{1} \\
x_{2} \\
x_{3} \\
x_{4} \\
x_{5} \\
x_{6} \\
x_{7} \\
x_{8}
\end{array}\right)
$$

where $x=x_{1}+x_{2} e_{1} e_{2}+x_{3} e_{1} e_{3}+x_{4} e_{1} e_{4}+x_{5} e_{1} e_{2} e_{3} e_{4}-x_{6} e_{3} e_{4}+x_{7} e_{2} e_{4}-x_{8} e_{2} e_{3}$, $A_{1}^{\prime}=-\frac{a_{1}+a_{2}+a_{3}}{2}, \quad A_{2}^{\prime}=\frac{a_{1}+a_{2}-a_{3}}{2}, \quad A_{3}^{\prime}=\frac{a_{1}-a_{2}+a_{3}}{2} \quad$ and $A_{4}^{\prime}=\frac{a_{1}-a_{2}-a_{3}}{2}$.

I) The case of $d=1$.

Put $X_{0}=1+e_{1} e_{2} e_{3} e_{4}={ }^{t}(10001000) \in V(8)$. We shall calculate the isoisotropy subalgebra $\mathfrak{g}_{X_{0}}$ of $\mathfrak{g}=\mathfrak{g l}(1) \oplus \mathfrak{o}(7, C)$ at $X_{0}$. Since

$$
\begin{array}{r}
d X_{0}+d \rho_{1}(A) X_{0}={ }^{t}\left(d-\frac{a_{1}+a_{2}+a_{3}}{2}, b_{12}-c_{34}, b_{13}+c_{24}, b_{14}-c_{23},\right. \\
\left.d+\frac{a_{1}+a_{2}+a_{3}}{2}, c_{12}-b_{34}, c_{13}+b_{24}, c_{14}-b_{23}\right)
\end{array}
$$

for $(d, A) \in g$, the isotropy subalgebra $\mathfrak{g}_{X_{0}}$ at $X_{0}$ is given as follows. 
$\mathfrak{g}_{X_{0}}=\left\{A=\left|\begin{array}{cccc|cccc}a_{1} & a_{12} & a_{13} & b_{14} & 0 & b_{12} & b_{13} & b_{14} \\ a_{21} & a_{2} & a_{23} & b_{24} & -b_{12} & 0 & b_{23} & b_{24} \\ a_{31} & a_{32} & a_{3} & b_{34} & -b_{13} & -b_{23} & 0 & b_{34} \\ -b_{23} & b_{13} & -b_{12} & 0 & -b_{14} & -b_{24} & -b_{34} & 0 \\ \hline 0 & b_{34} & -b_{24} & b_{23} & -a_{1} & -a_{21} & -a_{31} & b_{23} \\ -b_{34} & 0 & b_{14} & -b_{13} & -a_{12} & -a_{2} & -a_{32} & -b_{13} \\ b_{24} & b_{14} & 0 & b_{12} & -a_{13} & -a_{23} & -a_{3} & b_{12} \\ -b_{23} & b_{13} & -b_{12} & 0 & -b_{14} & -b_{24} & -b_{34} & 0\end{array}\right| a_{1}+a_{2}+a_{3}=0\right\}$

Since $\operatorname{dim} \mathfrak{g}_{X_{0}}=14=\operatorname{dim} G L(1) \times \operatorname{Spin}(7)-\operatorname{dim} V(8)$, it is a P.V. Let $S$ be the element of $G L(8)$ defined by

$$
S^{-1}=\left|\begin{array}{c|c|c|c}
0 & \begin{array}{c}
\frac{1}{2} \\
1
\end{array} & 0 & -\frac{1}{2} \\
\hdashline I_{3} & 0 & 0 & 0 \\
\hline 0 & 0 & -I_{3} & 0
\end{array}\right|, \quad S=\left|\begin{array}{c|c|c|c}
0 & 0 & I_{3} & 0 \\
\hline 1 & \frac{1}{2} & 0 & 0 \\
\hline 0 & 0 & 0 & -I_{3} \\
\hline-1 & \frac{1}{2} & 0 & 0
\end{array}\right| \in G L(8)
$$

Then we have

$$
S^{-1} A S=\left(\begin{array}{rr|rrr|rrr}
0 & 0 & \multicolumn{3}{|c|}{0} & \multicolumn{3}{|c}{0} \\
\hline 0 & 0 & -2 b_{23} & 2 b_{13} & -2 b_{12} & 2 b_{14} & 2 b_{24} & 2 b_{34} \\
\cline { 2 - 7 } & b_{14} & a_{1} & a_{12} & a_{13} & 0 & -b_{12} & -b_{13} \\
0 & b_{24} & a_{21} & a_{2} & a_{23} & b_{12} & 0 & -b_{23} \\
& b_{34} & a_{31} & a_{32} & a_{3} & b_{13} & b_{23} & 0 \\
\hline & -b_{23} & 0 & -b_{34} & b_{24} & -a_{1} & -a_{21} & -a_{31} \\
0 & b_{13} & b_{34} & 0 & -b_{14} & -a_{12} & -a_{2} & -a_{32} \\
& -b_{12} & -b_{24} & b_{14} & 0 & -a_{13} & -a_{23} & -a_{3}
\end{array}\right)
$$

By (1.8) in Example 30 in $\S 1$, this is an element of $\left(\mathfrak{g}_{2}\right)$ i.e. $\mathfrak{g}_{X_{0}} \cong\left(\mathfrak{g}_{2}\right)$, and hence it is a regular P.V. by Proposition 25 in $\S 4$. The relative invariant is the quadratic form $q(x)$. J. Igusa completed the orbital decomposition of this triplet (See [2]).

Proposition 25. A triplet $(G L(1) \times \operatorname{Spin}(7), \square \otimes \operatorname{spin}$ rep., $V(1) \otimes$ $V(8)$ ) is a regular $P . V$. and its generic isotropy subgroup is locally isomorphic to $\left(G_{2}\right)$. The relative invariant is a quadratic form.

II) The case of $d=2$.

We identify $V=V(8) \otimes V(2)$ with $8 \times 2$ matrices $M(8,2)$. Put $X_{0}$ $=\left(\begin{array}{l}10000000 \\ 00001000\end{array}\right) \in V$. We shall calculate the isotropy subalgebra $g_{X_{0}}$ of 
$g=\mathfrak{o}(7, C) \oplus \operatorname{gr}(2)$ at $X_{0} . \quad$ From (5.31), we have

$$
\begin{aligned}
& d \rho_{1}(A) X_{0}+X_{0}^{t} D=\left(\begin{array}{c|ccc}
d_{11}-\frac{a_{1}+a_{2}+a_{3}}{2} & b_{12} & b_{13} & b_{14} \\
d_{21} & -c_{34} & c_{24} & -c_{23}
\end{array}\right. \\
& \left.\mid \begin{array}{c|ccc}
d_{12} & d_{22}+\frac{a_{1}+a_{2}+a_{3}}{2} & b_{24} & -b_{23} \\
c_{12} & c_{13} & c_{14}
\end{array}\right) \in V,
\end{aligned}
$$

where $A \in \mathfrak{v}(7, C)$ and $D=\left(d_{i j}\right) \in \mathfrak{g} \mathfrak{l}(2)$. Hence the isotropy subalgebra $\mathfrak{g}_{X_{0}}$ is given by

$$
\begin{aligned}
\mathfrak{g}_{X_{0}} & =\left\{\left(\begin{array}{c|c|c}
\frac{2 \alpha I_{3}+A_{0}}{0} & \frac{0}{0} & 0 \\
\hline 0 & \frac{-2 \alpha I_{3}-{ }^{t} A_{0}}{0} & \frac{0}{0}
\end{array}\right) \oplus\left(\begin{array}{cc}
3 \alpha & 0 \\
0 & -3 \alpha
\end{array}\right) \mid A_{0} \in \mathfrak{g l}(3)\right\} \\
& \cong \mathfrak{g l}(3) \oplus \mathfrak{o}(2) .
\end{aligned}
$$

Since $\operatorname{dim} \mathfrak{g}_{X_{0}}=9=\operatorname{dim} \operatorname{Spin}(14) \times G L(2)-\operatorname{dim} V(8) \otimes V(2)$, it is a regular P.V. by Proposition 25 in $\S 4$. Since Spin (7) $\longleftrightarrow S O(8)$ by the spin representation, there exists an irreducible relative invariant of degree 4 by Proposition 23.

Proposition 26. A triplet $\left(\operatorname{Spin}(7) \times G L(2)\right.$, spin rep. $\otimes \Lambda_{1}, V(8) \otimes$ $V(2))$ is a regular P.V. and its generic isotropy subgroup is locally isomorphic to $S L(3) \times O(2)$. The relative invariant is of degree 4.

III) The case of $d=3$.

We identify $V=V(8) \otimes V(3)$ with $8 \times 3$ matrices $M(8,3)$, and put $X_{0}=\left(\begin{array}{l}10000000 \\ 00001000 \\ 01000100\end{array}\right) \in V$. Then from (5.31), we have

$$
\begin{aligned}
& d \rho_{1}(A) X_{0}+X_{0}^{t} D \\
& =\left(\begin{array}{c|c|c|c}
A_{1}^{\prime}+d_{11} & b_{12}+d_{13} & b_{13} & b_{14} \\
d_{21} & -c_{34}+d_{23} & c_{24} & -c_{23} \\
c_{34}-c_{12}+d_{31} & A_{2}^{\prime}+d_{33} & a_{32}+b_{14} & -c_{24}-a_{13}
\end{array}\right. \\
& \left.\begin{array}{c|c|c|c}
d_{12} & d_{13}-b_{34} & b_{24} & -b_{23} \\
-A_{1}^{\prime}+d_{22} & c_{12}+d_{23} & c_{13} & c_{14} \\
b_{34}-b_{12}+d_{32} & d_{33}-A_{2}^{\prime} & c_{14}-a_{23} & a_{31}-b_{24}
\end{array}\right) \in V .
\end{aligned}
$$

Hence the isotropy subalgebra $g_{X_{0}}=\left\{(A, D) \mid d \rho_{1}(A) X_{0}+X_{0}{ }^{t} D=0\right\} \quad$ of 
$\mathfrak{g}=\mathfrak{o}(7, C) \oplus \mathfrak{g l}(3)$ at $X_{0}$ is given as follows.

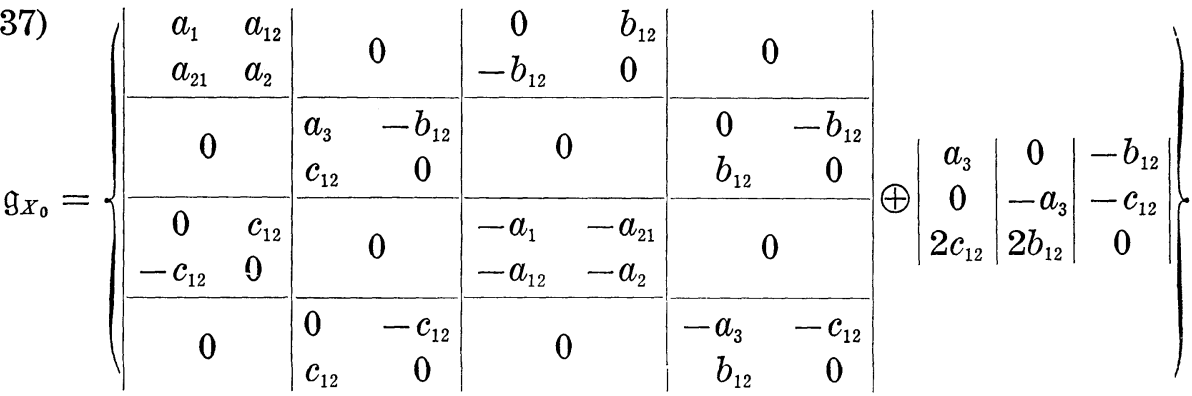

$$
\begin{aligned}
& \cong \mathfrak{g l}(2) \oplus \mathfrak{D}(3) \text {. }
\end{aligned}
$$

with $a_{1}+a_{2}=a_{3}$

Since $\operatorname{dim} \mathfrak{g}_{X_{0}}=6=\operatorname{dim} \operatorname{Spin}(7) \times G L(3)-\operatorname{dim} V(8) \otimes V(3)$, it is a regular P.V. by Proposition 25 in $\S 4$. Since Spin $(7) \smile S O(8)$ by the spin representation, there exists an irreducible relative invariant polynomial of degree 6 by Proposition 23.

Proposition 27. A triplet $\left(\operatorname{Spin}(7) \times G L(3)\right.$, spin rep. $\otimes \Lambda_{1}, V(8) \otimes$ $V(3)$ ) is a regular P.V. and its generic isotropy subgroup is locally isomorphic to $S L(2) \times O(3)$. The relative invariant is of degree 6 .

IV) The case of $d=4$.

Assume that this triplet is a P.V. and let $H$ be a generic isotropy subgroup. Then we have $\operatorname{dim} H=\operatorname{dim} \operatorname{Spin}(7) \times G L(4)-\operatorname{dim} V(8) \otimes$ $V(4)=5$. Since $S p i n(7) \smile S O(8)$ by the spin representation, we may consider that this P.V. is contained in a regular P.V. $(S O(8) \times G L(4)$, $\left.\Lambda_{1} \otimes \Lambda_{1}, V(8) \otimes V(4)\right)$ and hence by Proposition 23 we have $H=\operatorname{Spin}(7)$ $\times G L(4) \cap S O(4) \times S O(4) \times\{ \pm 1\} \supset S O(4)$ (See (20) in $\S 5$ ). This implies that $\operatorname{dim} H \geqq \operatorname{dim} S O(4)=6$, i.e., a contradiction, and hence we obtain the following proposition.

Proposition 28. A triplet $\left(\operatorname{Spin}(7) \times G L(4)\right.$, spin rep. $\otimes \Lambda_{1}, V(8) \otimes$ $V(4))$ is not a P.V.

$$
\left(\operatorname{Spin}(7) \times G L(2), \text { spin rep. } \otimes 2 \Lambda_{1}, V(8) \otimes V(3)\right) .
$$

Assume that this triplet is a P.V., and let $H$ be its generic isotropy subgroup. Then we have $\operatorname{dim} H=\operatorname{dim} \operatorname{Spin}(7) \times G L(2)-\operatorname{dim} V(8) \otimes$ $V(3)=1$. Since $\left(S L(2), 2 \Lambda_{1}, V(3)\right) \cong\left(S O(3), \Lambda_{1}, V(3)\right)$, we may consider that this triplet is contained in $\left(\operatorname{Spin}(7) \times G L(3)\right.$, spin rep. $\otimes \Lambda_{1}, V(8) \otimes$ $V(3)$ ) and hence by Proposition $27, H^{0}=S L(2) \times O(3) \cap S p i n(7) \times S O(3)$ 
$\supset S O(3)$ where $H^{0}$ denotes the connected component of $H$. This implies that $\operatorname{dim} H \geqq \operatorname{dim} S O(3)=3$ i.e. a contradiction, and hence we obtain the following proposition.

Proposition 29. A triplet $\left(\operatorname{Spin}(7) \times G L(2)\right.$, spin rep. $\otimes 2 \Lambda_{1}, V(8)$ $\otimes V(3))$ is not a P.V.

(24) $\quad\left(G L(1) \times S p i n(7) \times S p(2), \square \otimes \operatorname{spin}\right.$ rep. $\left.\otimes \Lambda_{1}, V(1) \otimes V(8) \otimes V(4)\right)$

We may consider that this triplet is contained in a triplet (Spin (7) $\times G L(4)$, spin rep. $\left.\otimes \Lambda_{1}, V(8) \otimes V(4)\right)$ which is not a P.V. by Proposition 28 , and hence it is not a P.V.

Proposition 30. A triplet $(G L(1) \times S p i n(7) \times S p(2), \square \otimes$ spin rep. $\left.\otimes \Lambda_{1}, V(1) \otimes V(8) \otimes V(4)\right)$ is not a P.V.

(25) $\quad\left(\operatorname{Spin}(10) \times G L(d)\right.$, half-spin rep. $\left.\otimes \Lambda_{1}, V(16) \otimes V(d)\right) \quad(1 \leq d \leq 3)$

First of all, we shall calculate the half-spin representation $d \rho_{1}$ of $\mathfrak{o}(10, C)$ by (5.29). The representation space $V(16)$ is spanned by 1 , $e_{i} e_{j}(1 \leq i<j \leq 5), e_{i} e_{j} e_{k} e_{\ell}(1 \leq i<j<k<\ell \leq 5)$. We may assume that an element $A$ in $\mathfrak{o}(10, C)$ is of the form (5.28). Then by (5.29) we have

1) $d \rho_{1}(A) \cdot 1=-\frac{a_{1}+a_{2}+a_{3}+a_{4}+a_{5}}{2}+\sum_{i<j} b_{i j} e_{i} e_{j}$

2) $d \rho_{1}(A) e_{k} e_{\ell}=\sum_{i \neq k} a_{i k} e_{i} e_{\ell}-\sum_{i \neq \ell} a_{i \ell} e_{i} e_{k}+\frac{a_{k}+a_{\ell}}{2} e_{k} e_{\ell}-\sum_{s \neq k, \ell} \frac{a_{s}}{2} e_{k} e_{\ell}$

$+\sum_{i<j} b_{i j} e_{i} e_{j} e_{k} e_{\ell}-c_{k \ell}(k<\ell)$

3) $d \rho_{1}(A) e_{k} e_{\ell} e_{m} e_{n}=a_{s k} e_{s} e_{\ell} e_{m} e_{n}-a_{s \ell} e_{s} e_{k} e_{m} e_{n}+a_{s m} e_{s} e_{k} e_{\ell} e_{n}-a_{s n} e_{s} e_{k} e_{\ell} e_{m}$

$+\frac{a_{k}+a_{\ell}+a_{m}+a_{n}-a_{s}}{2} e_{k} e_{\ell} e_{m} e_{n}-c_{k \ell} e_{m} e_{n}+c_{k m} e_{\ell} e_{n}-c_{k n} e_{\ell} e_{m}$

$-c_{\ell m} e_{k} e_{n}+c_{\ell n} e_{k} e_{m}-c_{m n} e_{k} e_{\ell}$ where $1 \leq k<\ell<m<n \leq 5$ and $\{s, k, \ell, m, n\}=\{1, \cdots, 5\}$.

Hence we have

$$
d \rho_{1}(A) x=\left(\begin{array}{l|l|l}
\alpha_{1} & \frac{\alpha_{2}}{\alpha_{3}} & \alpha_{4}
\end{array}\right)\left(\begin{array}{c}
x_{1} \\
\vdots \\
x_{16}
\end{array}\right)
$$

where $\alpha_{1}, \cdots, \alpha_{4}$ are given as follows. 


$$
\begin{aligned}
& \alpha_{1}=\left|\begin{array}{r|r|r|r|r|r|r|r}
A_{1} & -c_{12} & -c_{13} & -c_{14} & -c_{15} & -c_{23} & -c_{24} & -c_{25} \\
b_{12} & A_{2} & a_{23} & a_{24} & a_{25} & -a_{13} & -a_{14} & -a_{15} \\
b_{13} & a_{32} & A_{3} & a_{34} & a_{35} & a_{12} & & \\
b_{14} & a_{42} & a_{43} & A_{4} & a_{45} & & a_{12} & \\
b_{15} & a_{52} & a_{53} & a_{54} & A_{5} & & & a_{12} \\
b_{23} & -a_{31} & a_{21} & & & A_{6} & a_{34} & a_{35} \\
b_{24} & -a_{41} & & a_{21} & & a_{43} & A_{7} & a_{45} \\
b_{25} & -a_{51} & & & a_{21} & a_{53} & a_{54} & A_{8}
\end{array}\right|, \\
& \alpha_{2}=\left|\begin{array}{r|r|r|r|r|r|r|r|}
-c_{34} & -c_{35} & -c_{45} & & & & \\
-a_{14} & -a_{15} & & -c_{34} & -c_{35} & -c_{45} & & \\
a_{13} & & -a_{15} & -c_{23} & c_{25} & & -c_{45} & c_{25} \\
c_{35} & \\
-a_{24} & -a_{25} & a_{14} & & -c_{23} & -c_{24} & -c_{34} & \\
a_{23} & & -c_{14} & -c_{15} & & & -c_{45} \\
a_{13} & & -c_{15} & \\
c_{35} & a_{23} & & c_{13} & c_{34}
\end{array}\right|,
\end{aligned}
$$

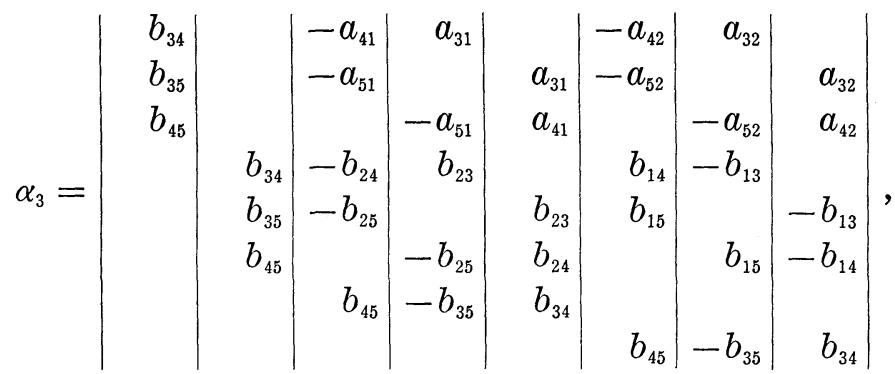

$$
\begin{aligned}
& \alpha_{4}=\mid \begin{array}{r|r|r|r|r|r|r|r}
A_{9} & a_{45} & -a_{35} & -c_{12} & & & -c_{15} & -c_{25} \\
a_{54} & A_{10} & a_{34} & & -c_{12} & & c_{14} & c_{24} \\
-a_{53} & a_{43} & A_{11} & & & -c_{12} & -c_{13} & -c_{23} \\
b_{12} & & & A_{12} & a_{45} & -a_{35} & a_{25} & -a_{15} \\
& b_{12} & & a_{54} & A_{13} & a_{34} & -a_{24} & a_{14} \\
& & b_{12} & -a_{53} & a_{43} & A_{14} & a_{23} & -a_{13} \\
b_{15} & -b_{14} & b_{13} & a_{52} & -a_{42} & a_{32} & A_{15} & a_{12} \\
b_{25} & -b_{24} & b_{23} & -a_{51} & a_{41} & -a_{31} & a_{21} & A_{16}
\end{array}
\end{aligned}
$$

where

$$
\begin{array}{ll}
2 A_{1}=-a_{1}-a_{2}-a_{3}-a_{4}-a_{5}, & 2 A_{2}=a_{1}+a_{2}-a_{3}-a_{4}-a_{5}, \\
2 A_{3}=a_{1}-a_{2}+a_{3}-a_{4}-a_{5}, & 2 A_{4}=a_{1}-a_{2}-a_{3}+a_{4}-a_{5}, \\
2 A_{5}=a_{1}-a_{2}-a_{3}-a_{4}+a_{5}, & 2 A_{6}=-a_{1}+a_{2}+a_{3}-a_{4}-a_{5}, \\
2 A_{7}=-a_{1}+a_{2}-a_{3}+a_{4}-a_{5}, & 2 A_{8}=-a_{1}+a_{2}-a_{3}-a_{4}+a_{5}, \\
2 A_{9}=-a_{1}-a_{2}+a_{3}+a_{4}-a_{5}, & 2 A_{10}=-a_{1}-a_{2}+a_{3}-a_{4}+a_{5},
\end{array}
$$




$$
\begin{array}{ll}
2 A_{11}=-a_{1}-a_{2}-a_{3}+a_{4}+a_{5}, & 2 A_{12}=a_{1}+a_{2}+a_{3}+a_{4}-a_{5}, \\
2 A_{13}=a_{1}+a_{2}+a_{3}-a_{4}+a_{5}, & 2 A_{14}=a_{1}+a_{2}-a_{3}+a_{4}+a_{5}, \\
2 A_{15}=a_{1}-a_{2}+a_{3}+a_{4}+a_{5}, & 2 A_{16}=-a_{1}+a_{2}+a_{3}+a_{4}+a_{5},
\end{array}
$$

and

$$
\begin{aligned}
x= & x_{1}+x_{2} e_{1} e_{2}+x_{3} e_{1} e_{3}+x_{4} e_{1} e_{4}+x_{5} e_{1} e_{5}+x_{6} e_{2} e_{3}+x_{7} e_{2} e_{4}+x_{8} e_{2} e_{5} \\
& +x_{9} e_{3} e_{4}+x_{10} e_{3} e_{5}+x_{11} e_{4} e_{5}+x_{12} e_{1} e_{2} e_{3} e_{4}+x_{13} e_{1} e_{2} e_{3} e_{5} \\
& +x_{14} e_{1} e_{2} e_{4} e_{5}+x_{15} e_{1} e_{3} e_{4} e_{5}+x_{16} e_{2} e_{3} e_{4} e_{5} .
\end{aligned}
$$

We identify $V(16)$ with $C^{16}$ by an isomorphisms $x \mapsto{ }^{t}\left(x_{1}, \cdots, x_{16}\right) \in C^{16}$.

I) The case of $d=1$.

Put $X_{0}=1+e_{1} e_{2} e_{3} e_{4}={ }^{t}(10 \cdots 010000) \in V(16)$. We shall calculate the isotropy subalgebra $\mathfrak{g}_{X_{0}}$ of $\mathfrak{g}=\mathfrak{g r}(1) \oplus \mathfrak{v}(10, C)$ at $X_{0}$. From (5.38), we have $a X_{0}+d \rho_{1}(A) X_{0}={ }^{t}\left(a+A_{1}, b_{12}-c_{34}, b_{13}+c_{24}, b_{14}-c_{23}, b_{15}, b_{23}-c_{14}\right.$, $\left.b_{24}+c_{13}, b_{25}, b_{34}-c_{12}, b_{35}, b_{45}, a+A_{12}, a_{54},-a_{53}, a_{52},-a_{51}\right) \in V(16)$ where $(a, A)$ $\in \mathfrak{g r}(1) \oplus \mathfrak{o}(10)$. Hence the isotropy subalgebra $\mathfrak{g}_{X_{0}}=\left\{(a, A) \mid a X_{0}+d \rho_{1}(A) X_{0}\right.$ $=0\}$ is given as follows.

$$
\mathfrak{g}_{X_{0}}=\left\{(a) \oplus\left|\begin{array}{ccccc|ccccc}
a_{1} & a_{12} & a_{13} & a_{14} & a_{15} & 0 & \alpha_{1} & \alpha_{2} & \alpha_{3} & 0 \\
a_{21} & a_{2} & a_{23} & a_{24} & a_{25} & -\alpha_{1} & 0 & \beta_{1} & \beta_{2} & 0 \\
a_{31} & a_{32} & a_{3} & a_{34} & a_{35} & -\alpha_{2} & -\beta_{1} & 0 & \beta_{3} & 0 \\
a_{41} & a_{42} & a_{43} & a_{4} & a_{45} & -\alpha_{3} & -\beta_{2} & -\beta_{3} & 0 & 0 \\
0 & 0 & 0 & 0 & 2 a & 0 & 0 & 0 & 0 & 0 \\
\hline 0 & \beta_{3} & -\beta_{2} & \beta_{1} & c_{15} & -a_{1} & -a_{21} & -a_{31} & -a_{41} & 0 \\
-\beta_{3} & 0 & \alpha_{3} & -\alpha_{2} & c_{25} & -a_{12} & -a_{2} & -a_{32} & -a_{42} & 0 \\
\beta_{2} & -\alpha_{3} & 0 & \alpha_{1} & c_{35} & -a_{13} & -a_{23} & -a_{3} & -a_{43} & 0 \\
-\beta_{1} & \alpha_{2} & -\alpha_{1} & 0 & c_{45} & -a_{14} & -a_{24} & -a_{34} & -a_{4} & 0 \\
-c_{15} & -c_{25} & -c_{35} & -c_{45} & 0 & -a_{15} & -a_{25}-a_{35}-a_{45} & -2 \alpha
\end{array}\right|\right.
$$

Since $\operatorname{dim} g_{X_{0}}=30=\operatorname{dim}(G L(1) \times \operatorname{Spin}(10))-\operatorname{dim} V(16)$, this triplet is a P.V. Since $\mathfrak{g}_{X_{0}} \not \subset \mathfrak{g l}(V)$, there is no relative invariant by Proposition 20 in $\S 4$. From (5.39) we have $\mathfrak{g}_{X_{0}} \cong(\mathfrak{g l}(1) \oplus \mathfrak{o}(7)) \oplus V(8)$ where $V(8)$ is the Lie algebra of the vector group of dimension eight. This space was investigated by J. Igusa (See [2]).

Proposition 31. A triplet (GL(1) $\times$ Spin (10), $\square \otimes$ half-spin rep., $V(1) \otimes V(16))$ is a P.V. and its generic isotropy subgroup is locally isomorphic to $(G L(1) \times \operatorname{Spin}(7)) \cdot\left(G_{a}\right)^{8}$. There is no relative invariant and 
hence it is not regular.

II) The case of $d=2$.

We identify $V=V(16) \otimes V(2)$ with $16 \times 2$ matrices $M(16,2)$. Then the action $d \rho$ of $\mathfrak{g}=\mathfrak{o}(10) \oplus \mathfrak{g l}(2)$ is given by $d \rho(A, D) X=d \rho_{1}(A) X+X^{t} D$ for $X \in V,(A, D) \in \mathfrak{g}$. Put

$$
X_{0}=\left(1+e_{1} e_{2} e_{3} e_{4}, e_{1} e_{5}+e_{2} e_{3} e_{4} e_{5}\right)={ }^{t}\left(\begin{array}{l}
10 \cdots 010000 \\
000010 \cdots 01
\end{array}\right) \in V
$$

Then from (5.38), we have

$$
\begin{aligned}
& d \rho(A, D) X_{0}={ }^{t}\left(\begin{array}{c|c|c|c|c|c}
d_{11}+A_{1} & b_{12}-c_{34} & b_{13}+c_{24} & b_{14}-c_{23} & d_{21}+b_{15} & b_{23}-c_{14} \\
d_{12}-c_{15} & a_{25} & a_{35} & a_{45} & d_{22}+A_{5} & -c_{45}
\end{array}\right. \\
& \mid \begin{array}{c|c|c|c|c}
b_{24}+c_{13} & b_{25} & b_{34}-c_{12} & b_{35} & b_{45} \\
c_{35} & a_{21}-c_{34} & -c_{25} & a_{31}+c_{24} & a_{41}-c_{23}
\end{array} \\
& \left.\mid \begin{array}{c|c|c|c|c}
d_{11}+A_{12} & a_{54} & -a_{53} & a_{52} & d_{21}-a_{51} \\
d_{12}-a_{15} & b_{23}+a_{14} & b_{24}-a_{13} & b_{34}+a_{12} & d_{22}+A_{16}
\end{array}\right)
\end{aligned}
$$

and hence the isotropy subalgebra $\mathfrak{g}_{X_{0}}=\left\{(A, D) \mid d \rho(A, D) X_{0}=0\right\}$ is given as follows.

$$
\begin{aligned}
& \mathfrak{g}_{X_{0}}=\left\{\begin{array}{ccccc|ccccc}
0 & a_{12} & a_{13} & a_{14} & d_{12} & 0 & a_{21} & a_{31} & a_{41} & -d_{21} \\
a_{21} & a_{2} & a_{23} & a_{24} & 0 & -a_{21} & 0 & -a_{14} & a_{13} & 0 \\
a_{31} & a_{32} & a_{3} & a_{34} & 0 & -a_{31} & a_{14} & 0 & -a_{12} & 0 \\
a_{41} & a_{42} & a_{43} & a_{4} & 0 & -a_{41} & -a_{13} & a_{12} & 0 & 0 \\
d_{21} & 0 & 0 & 0 & 2 d_{11} & d_{21} & 0 & 0 & 0 & 0 \\
\hline 0 & -a_{12} & -a_{13} & -a_{14} & d_{12} & 0 & -a_{21} & -a_{31} & -a_{41} & -d_{21} \\
a_{12} & 0 & a_{41} & -a_{31} & 0 & -a_{12} & -a_{2} & -a_{32} & -a_{42} & 0 \\
a_{13} & -a_{41} & 0 & a_{21} & 0 & -a_{13} & -a_{23} & -a_{3} & -a_{43} & 0 \\
a_{14} & a_{31} & -a_{21} & 0 & 0 & -a_{14} & -a_{24} & -a_{34} & -a_{4} & 0 \\
-d_{12} & 0 & 0 & 0 & 0 & -d_{12} & 0 & 0 & 0 & -2 d_{11}
\end{array} \mid\right. \\
&\left.\oplus\left(\begin{array}{cc}
d_{11} & d_{12} \\
d_{21} & -d_{11}
\end{array}\right\}\right\} \quad \text { with } a_{2}+a_{3}+a_{4}=0 .
\end{aligned}
$$

Since $\operatorname{dim} \mathfrak{g}_{X_{0}}=17=\operatorname{dim} \operatorname{Spin}(10) \times G L(2)-\operatorname{dim} V(16) \otimes V(2)$, it is a P.V. Let $A$ be the $10 \times 10$ matrix in (5.40) and put $S$ in $G L(10)$ as follows. 

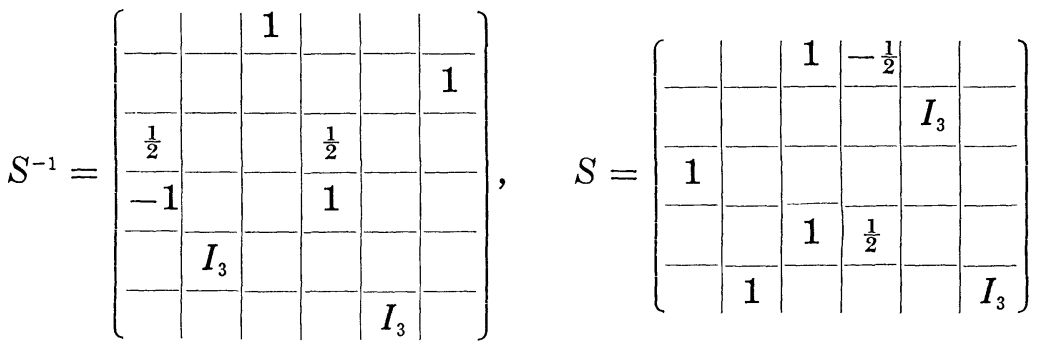

where $I_{3}$ is the identity matrix of size three. Then by simple calculation, we have

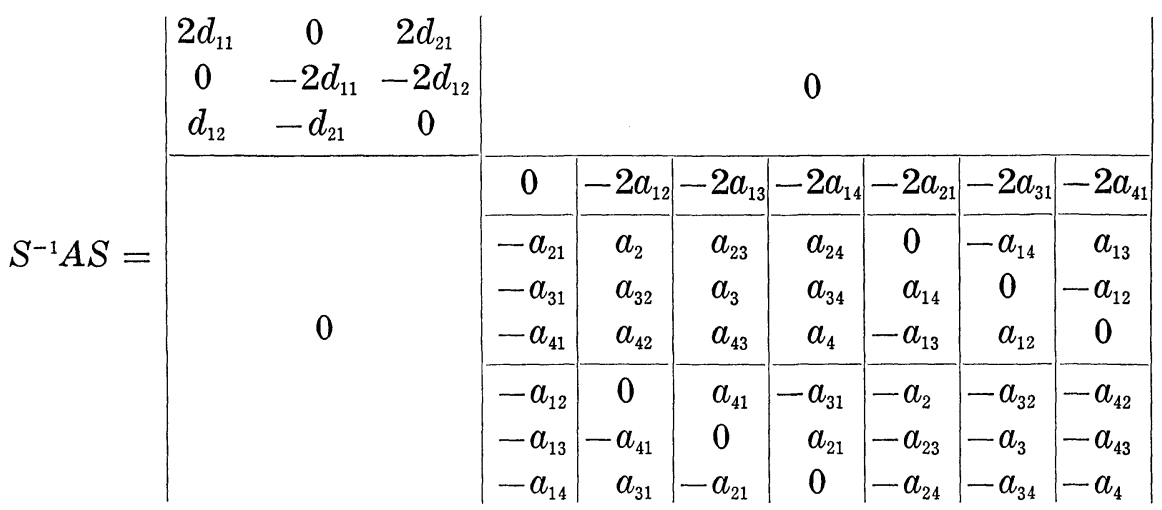

with $a_{2}+a_{3}+a_{4}=0$.

Therefore we have $g_{X_{0}} \cong\left(g_{2}\right) \oplus \mathfrak{g l}(2)$ (See (1.8) in $\S 1$ ), and hence this triplet is regular by Proposition 25 in $\S 4$. Put

$$
X_{0}^{\prime}=\left(1+e_{1} e_{2} e_{3} e_{4}, e_{1} e_{2}+e_{2} e_{3} e_{4} e_{5}\right)={ }^{t}\left(\begin{array}{c}
10 \ldots 010000 \\
010 \ldots .01
\end{array}\right) \in V
$$

Then from (5.38), we have

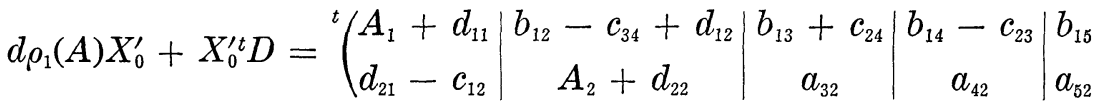

$$
\begin{aligned}
& \left|\begin{array}{c|c}
b_{23}-c_{14} & b_{24}+c_{13} \\
-a_{31}-c_{45} & c_{35}-a_{41}
\end{array}\right|-b_{51}-c_{34}\left|\begin{array}{c}
b_{34}-c_{12} \\
-c_{25}
\end{array}\right| \begin{array}{c|c}
b_{35} \\
c_{24}
\end{array} \mid-c_{23} \\
& \left.\mid \begin{array}{c|c|c|c|c}
A_{12}+d_{11} & a_{54} & -a_{53} & a_{52} & d_{12}-a_{51} \\
b_{34}-a_{15}+d_{21} & b_{35}+a_{14} & b_{45}-a_{13} & a_{12} & d_{22}+A_{16}
\end{array}\right)
\end{aligned}
$$

and hence the isotropy subalgebra $g_{X_{0}^{\prime}}$ at $X_{0}^{\prime}$ is given as follows. 


$$
\begin{aligned}
& \mathfrak{g}_{X_{0}}=\left\{\begin{array}{c|c|c|c|c|c|c|c|c|c}
d_{11}+d_{22} & 0 & 0 & 0 & 2 c_{12} & 0 & 2 c_{34} & 0 & 0 & 0 \\
a_{21} & -2 d_{22} & a_{23} & a_{24} & a_{25} & -2 c_{34} & 0 & c_{14} & -c_{13} & 0 \\
-c_{45} & 0 & a_{3} & a_{34} & a_{35} & 0 & -c_{14} & 0 & c_{12} & 0 \\
c_{35} & 0 & a_{43} & a_{4} & a_{45} & 0 & c_{13} & -c_{12} & 0 & 0 \\
-c_{34} & 0 & 0 & 0 & 2 d_{11} & 0 & 0 & 0 & 0 & 0 \\
\hline 0 & c_{12} & c_{13} & c_{14} & c_{15} & -d_{11}-d_{22} & -a_{21} & c_{45} & -c_{35} & c_{34} \\
-c_{12} & 0 & 0 & 0 & 0 & 0 & 2 d_{22} & 0 & 0 & 0 \\
-c_{13} & 0 & 0 & c_{34} & c_{35} & 0 & -a_{23} & -a_{3} & -a_{43} & 0 \\
-c_{14} & 0 & -c_{34} & 0 & c_{45} & 0 & -a_{24} & -a_{34} & -a_{4} & 0 \\
-c_{15} & 0 & -c_{35} & -c_{45} & 0 & -2 c_{12} & -a_{25} & -a_{35} & -a_{45} & -2 d_{11}
\end{array}\right\} \\
&\left.\oplus\left(\begin{array}{cc}
d_{11} & -c_{34} \\
c_{12} & d_{22}
\end{array}\right\}\right\} \text { with } a_{3}+a_{4}=d_{22}-d_{11}
\end{aligned}
$$

Since $\operatorname{dim} \mathrm{g}-\operatorname{dim} \mathfrak{g}_{X_{0}^{\prime}}=49-18=31$, the orbit of $X_{0}^{\prime}$ is of codimension one. For $(A, D) \in \mathfrak{g}_{X^{\prime}}$, the trace $\operatorname{tr}_{V}(A, D)$ in $V$ is $16\left(d_{11}+d_{22}\right)$ and the trace $\operatorname{tr} \operatorname{adg}_{X_{0}^{\prime}} A$ of the adjoint representation is by simple calculation $-14\left(d_{11}+d_{22}\right)$ and hence

$$
\operatorname{deg} f=\frac{16\left(d_{11}+d_{22}\right)-14\left(d_{11}+d_{22}\right)}{16\left(d_{11}+d_{22}\right)} \times 32=4
$$

by Proposition 15 in $\S 4$. The explicit form of this irreducible relative invariant $f(x)$ of degree 4 is given by Kawahara (See [13]).

Proposition 32. A triplet (Spin $(10) \times G L(2)$, half-spin rep. $\otimes \Lambda_{1}$, $V(16) \otimes V(2))$ is a regular P.V. and its generic isotropy subgroup is locally isomorphic to $\left(G_{2}\right) \times S L(2)$. The relative invariant is a quartic form.

III) The case of $d=3$.

We identify $V=V(16) \otimes V(3)$ with $16 \times 3$ matrices $M(16,3)$. Then the action $d \rho$ of $\mathfrak{g}=\mathfrak{o}(10) \oplus \mathrm{gr}(3)$ is given by $d \rho(A, D) X=d \rho_{1}(A) X+X^{t} D$ for $(A, D) \in \mathfrak{g}, X \in M(16,3)$.

Put

$$
\begin{aligned}
X_{0} & =\left(1+e_{1} e_{2} e_{3} e_{4}, e_{1} e_{5}+e_{2} e_{3} e_{4} e_{5}, e_{1} e_{2}+e_{1} e_{3} e_{4} e_{5}\right) \\
& =\left(\begin{array}{l}
10 \cdots 010000 \\
000010 \cdots 01 \\
010 \cdots 0.010
\end{array}\right) \in M(16,3)
\end{aligned}
$$

We shall calculate the isotropy subalgebra $g_{X_{0}}$ at $X_{0}$. By (5.38) we have 


$$
\begin{aligned}
& d \rho(A, D) X_{0}=d \rho_{1}(A) X_{0}+X_{0}{ }^{t} D \\
& =\left(\begin{array}{c|c|c|c|c}
A_{1}+d_{11} & b_{12}-c_{34}+d_{13} & b_{13}+c_{24} & b_{14}-c_{23} & b_{15}+d_{12} \\
d_{21}-c_{15} & a_{25}+d_{23} & a_{35} & a_{45} & A_{5}+d_{22} \\
d_{31}-c_{12} & A_{2}+d_{33} & a_{32}-c_{45} & a_{42}+c_{35} & a_{52}-c_{34}+d_{32}
\end{array}\right. \\
& \begin{array}{|c|c|c|c|c|c}
b_{23}-c_{14} & b_{24}+c_{13} & b_{25} & b_{34}-c_{12} & b_{35} & b_{45} \\
-c_{45} & c_{35} & a_{21}-c_{34} & -c_{25} & a_{31}+c_{24} & a_{41}-c_{23} \\
-a_{31} & -a_{41} & -a_{51} & -c_{15} & c_{14} & -c_{13}
\end{array} \\
& \begin{array}{l|l|l|l|l}
A_{12}+d_{11} & a_{54} & -a_{53} & d_{13}+a_{52} & d_{12}-a_{51}
\end{array} \\
& \left.\begin{array}{c|c|c|c|c}
d_{21}-a_{15} & b_{23}+a_{14} & b_{24}-a_{13} & b_{34}+a_{12}+d_{23} & A_{16}+d_{22} \\
b_{34}+a_{25}+d_{31} & b_{35}-a_{24} & b_{45}+a_{23} & A_{15}+d_{33} & d_{32}+a_{21}
\end{array}\right)
\end{aligned}
$$

and hence the isotropy subalgebra $\mathfrak{g}_{X_{0}}=\left\{(A, D) \mid d \rho(A, D) X_{0}=0\right\}$ is given as follows.

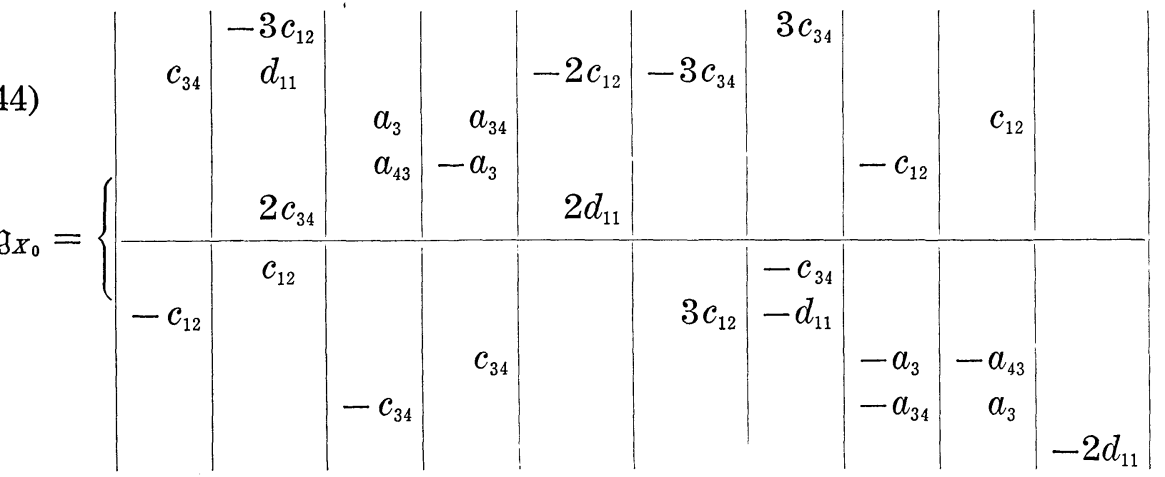

$$
\begin{aligned}
& \left.\oplus\left[\begin{array}{l|r|r}
d_{11} & -2 c_{34} \\
c_{12} & -d_{11} & 2 c_{12}
\end{array}\right]\right\} \\
& \cong\left\{\left(\begin{array}{cc}
a_{3}+d_{11} / 2 & a_{34} \\
a_{43} & -a_{3}-d_{11} / 2
\end{array}\right) \oplus\left[\begin{array}{ccc}
d_{11} & -2 c_{34} \\
& -d_{11} & 2 c_{12} \\
c_{12}-c_{34} & 0
\end{array}\right]\right\}=\operatorname{gl}(2) \oplus \mathfrak{o}(3) \text {. }
\end{aligned}
$$

Since $\operatorname{dim} g_{X_{0}}=6=\operatorname{dim} \operatorname{Spin}(10) \times G L(3)-\operatorname{dim} V(16) \otimes V(3)$, it is a P.V. and by Proposition 25 in $\S 4$, it is regular. Put

$$
\begin{aligned}
& X_{0}^{\prime}=\left(1+e_{1} e_{2} e_{3} e_{4}, e_{1} e_{5}+e_{2} e_{3} e_{4} e_{5}, e_{1} e_{2}+e_{3} e_{5}+e_{1} e_{2} e_{4} e_{5}\right)
\end{aligned}
$$

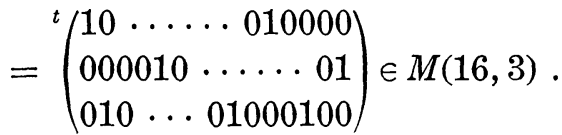

By (5.38), we have 


$$
\begin{aligned}
d \rho(A, D) X_{0}^{\prime}= & d \rho_{1}(A) X_{0}^{\prime}+X_{0}^{\prime t} D \\
= & \left(\begin{array}{c|c|c|c|c|}
A_{1}+d_{11} & b_{12}-c_{34}+d_{13} & b_{13}+c_{24} & b_{14}-c_{23} \\
d_{21}-c_{15} & a_{25}+d_{23} & a_{35} & a_{45} \\
d_{31}-c_{12}-c_{35} & A_{2}-c_{45}+d_{33} & a_{32}-a_{15} & a_{42}+c_{25} \\
b_{15}+d_{12} & b_{23}-c_{14} & b_{24}+c_{13} & b_{25} \\
A_{5}+d_{22} & -c_{45} & c_{35} & a_{21}-c_{34} \\
a_{52}+a_{13}-c_{24}+d_{32} & -a_{31}-a_{15} & -a_{41}-c_{15} & a_{23}+c_{14}-a_{51}
\end{array}\right. \\
& \left.\mid \begin{array}{c|c|c|c|c|}
b_{34}-c_{12} & b_{35}+d_{13} & b_{45} & A_{12}+d_{11} \\
-c_{25} & a_{31}+c_{24}+d_{23} & a_{41}-c_{23} & d_{21}-a_{15} \\
a_{45} & A_{10}+d_{33} & a_{43}-c_{12} & b_{34}+d_{31}-a_{35} \\
a_{54} & d_{13}-a_{53} & a_{52} & d_{12}-a_{51} \\
b_{23}+a_{14} & b_{24}-a_{13}+d_{23} & a_{12}+b_{34} & A_{16}+d_{22} \\
b_{35}+b_{12}+a_{34} & b_{45}+A_{14}+d_{33} & a_{32}-b_{14} & d_{32}-b_{24}-a_{31}
\end{array}\right)
\end{aligned}
$$

and hence the isotropy subalgebra $\mathfrak{g}_{X_{0}^{\prime}}=\left\{(A, D) \mid d \rho(A, D) X_{0}^{\prime}=0\right\}$ is given as follows.

$$
\begin{aligned}
\text { (5.45) } & \left\{\begin{array}{c|c|c|c|c|c|c|c|c|c|}
0 & 0 & -\alpha_{3} & -\alpha_{2} & 0 & 0 & \beta_{2} & -\alpha_{3} & 0 & -\alpha_{1} \\
\beta_{1}+\beta_{2} & 2 d & \alpha_{1}-\alpha_{2} & \beta_{3} & -\alpha_{3} & -\beta_{2} & 0 & \alpha_{2} & -2 \alpha_{3} & 0 \\
\alpha_{3} & 0 & 0 & \beta_{1}-\beta_{2} & 0 & \alpha_{3} & -\alpha_{2} & 0 & 0 & -\beta_{1} \\
0 & 0 & 0 & -2 d & 0 & 0 & 2 \alpha_{3} & 0 & 0 & 0 \\
\alpha_{1} & 0 & \beta_{1} & 0 & 2 d & \alpha_{1} & 0 & \beta_{1} & 0 & 0 \\
\hline 0 & 0 & 2 \alpha_{3} & \alpha_{2} & 0 & 0 & -\beta_{1}-\beta_{2} & -\alpha_{3} & 0 & -\alpha_{1} \\
0 & 0 & 0 & -2 \alpha_{3} & 0 & 0 & -2 d & 0 & 0 & 0 \\
-2 \alpha_{3} & 0 & 0 & \beta_{1}+\beta_{2} & 0 & \alpha_{3} & \alpha_{2}-\alpha_{1} & 0 & 0 & -\beta_{1} \\
-\alpha_{2} & 2 \alpha_{3} & -\beta_{1}-\beta_{2} & 0 & 0 & \alpha_{2} & -\beta_{3} & \beta_{2}-\beta_{1} & 2 d & 0 \\
0 & 0 & 0 & 0 & 0 & 0 & \alpha_{3} & 0 & 0 & -2 d
\end{array}\right) \\
& \left.\oplus\left[\begin{array}{cc|c}
d & \alpha_{1} & \beta_{1} \\
0 & -d & \alpha_{3} \\
0 & -\alpha_{3} & -d
\end{array}\right]\right\}
\end{aligned}
$$

Since $\operatorname{dim} \operatorname{Spin}(10) \times G L(3)-\operatorname{dim} g_{X_{0}^{\prime}}=54-7=47$, the orbit of $X_{0}^{\prime}$ is of codimension one, and the $\operatorname{trace}^{\operatorname{tr}_{V}}(A, D)$ on $V$ is $16(d-d-d)=-16 d$

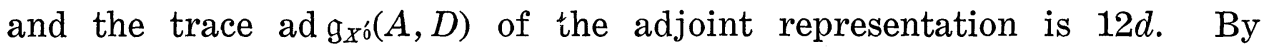
Proposition 15 in $\S 4$, the degree of the irreducible relative invariant polynomial $f(x)$ is given by $\operatorname{deg} f=((-16 d+12 d) /-16 d) \times 48=12$. The orbital decomposition of this space is completed by Kawahara (See [13]).

Proposition 33. A triplet (Spin(10) $\times G L(3)$, half-spin rep. $\otimes \Lambda_{1}$, $V(16) \otimes V(3))$ is a regular $P . V$. and its generic isotropy subgroup is locally 
isomorphic to $S L(2) \times O(3)$. The relative invariant is of degree 12 .

$\left(\operatorname{Spin}(10) \times G L(2)\right.$, half-spin rep. $\left.\otimes 2 \Lambda_{1}, V(16) \otimes V(3)\right)$.

Assume that this triplet is a P.V. and let $H$ be its generic isotropy subgroup. Then we have $\operatorname{dim} H=\operatorname{dim} \operatorname{Spin}(10) \times G L(2)-\operatorname{dim} V(16) \otimes V(3)$ $=1$. Since we may consider that this triplet is contained in (Spin (10) $\times$ $G L(3)$, half-spin rep. $\left.\otimes \Lambda_{1}, V(16) \otimes V(3)\right)$, we have $H \supset S p i n(10) \times G L(2)$ $\cap S L(2) \times S O(3) \supset S L(2)$ by (5.44) and hence $\operatorname{dim} H \geqq \operatorname{dim} S L(2)=3$, i.e., a contradiction. Thus we obtain the following proposition.

Proposition 34. A triplet $\left(\operatorname{Spin}(10) \times G L(2)\right.$, half-spin rep. $\otimes 2 \Lambda_{1}$, $V(16) \otimes V(3))$ is not a P.V.

$$
\left(\operatorname{Spin}(9) \times G L(d), \text { spin rep. } \otimes \Lambda_{1}, V(16) \otimes V(d)\right) \quad(1 \leq d \leq 2)
$$

Put $v_{1}=e_{5}-f_{5}={ }^{t}(000010000-1)$. Then $\mathfrak{o}(9, C)=\left\{A \in \mathfrak{o}(10, C) \mid A v_{1}=0\right\}$. We may assume that an element $A$ of $\mathfrak{o}(10, C)$ is of the form (5.28). Then $A$ is in $\mathrm{o}(9, C)$ if and only if $A v_{1}={ }^{t}\left(a_{15}+b_{15}, a_{25}+b_{25}, a_{35}+b_{35}\right.$, $\left.a_{45}+b_{45}, a_{5}, c_{15}-a_{51}, c_{25}-a_{52}, c_{35}-a_{53}, c_{45}-a_{54},-a_{5}\right)=0$ i.e. $a_{i 5}=-b_{i 5}$, $a_{5 i}=c_{i 5}, a_{5}=0(1 \leq i \leq 4)$.

I) The case of $d=1$.

Put $X_{0}=1+e_{1} e_{2} e_{3} e_{4}=(10 \cdots 010000) \in V(16)$. Then by (5.38) we have $a X_{0}+d \rho_{1}(A) X_{0}={ }^{t}\left(a-\left(a_{1}+a_{2}+a_{3}+a_{4}\right) / 2, b_{12}-c_{34}, b_{13}+c_{24}, b_{14}-\right.$ $c_{23}, b_{15}, b_{23}-c_{14}, b_{24}+c_{13}, b_{25}, b_{34}-c_{12}, b_{35}, b_{45}, a+\left(a_{1}+a_{2}+a_{3}+a_{4}\right) / 2$, $\left.a_{54},-a_{53}, a_{52},-a_{51}\right)$ with $a_{5}=0, a_{i 5}=-b_{i 5}, a_{5 i}=c_{i 5}(1 \leq i \leq 4)$ and $(a, A)$ $\in \mathfrak{g l}(1) \oplus \mathfrak{o}(9)$. Hence the isotropy subalgebra $\mathfrak{g}_{x_{0}}=\left\{(a, A) \mid a X_{0}+d \rho_{1}(A) X_{0}\right.$ $=0\}$ is given as follows.

$$
\mathfrak{g}_{X_{0}}=\left\{(0) \oplus \begin{array}{c|c|c|c|c|c|c|c|c|c|c|}
a_{31} & a_{32} & a_{3} & a_{34} & 0 & -b_{13} & -b_{23} & 0 & b_{34} & 0 \\
a_{41} & a_{42} & a_{43} & a_{4} & 0 & -b_{14} & -b_{24} & -b_{23} & 0 & 0 \\
0 & 0 & 0 & 0 & 0 & 0 & 0 & 0 & 0 & 0 \\
\hline 0 & b_{34} & -b_{24} & b_{23} & 0 & -a_{1} & -a_{21} & -a_{31} & -a_{41} & 0 \\
-b_{34} & 0 & b_{14} & -b_{13} & 0 & -a_{12} & -a_{2} & -a_{32} & -a_{42} & 0 \\
b_{24} & -b_{14} & 0 & b_{12} & 0 & -a_{13} & -a_{23} & -a_{3} & -a_{43} & 0 \\
-b_{23} & b_{13} & -b_{12} & 0 & 0 & -a_{14} & -a_{24} & -a_{34} & -a_{4} & 0 \\
0 & 0 & 0 & 0 & 0 & 0 & 0 & 0 & 0 & 0
\end{array} \mid\right.
$$


Since $\operatorname{dim} \mathfrak{g}_{X_{0}}=21=\operatorname{dim} G L(1) \times \operatorname{Spin}(9)-\operatorname{dim} V(1) \otimes V(16)$, it is a P.V. and by Proposition 25 in $\S 4$, it is regular. This space was investigated by J. Igusa (See [2]).

The relative invariant is a quadratic form $q(x)$, and the explicit form which is due to J. Igusa, is given as follows.

$$
q(x)=x_{0} y_{0}+\operatorname{Pff}\left(x_{i j}\right)+\sum_{i} x_{i} y_{i}
$$

where

$$
x=x_{0}+\sum_{i<j \leq 4} x_{i j} e_{i} e_{j}+y_{0} e_{1} e_{2} e_{3} e_{4}+\left(\sum_{i} x_{i} e_{i}+\sum_{i} y_{i} e_{i}^{*}\right) e_{5}
$$

and

$$
e_{i} e_{i}^{*}=e_{1} e_{2} e_{3} e_{4} e_{5} \quad(1 \leq i \leq 4) .
$$

Proposition 35. A triplet $(G L(1) \times \operatorname{Spin}(9), \square \otimes \operatorname{spin}$ rep., $V(1) \otimes$ $V(16)$ ) is a regular P.V. and its generic isotropy subgroup is locally isomorphic to Spin(7). The relative invariant is a quadratic form.

II) The case of $d=2$.

Assume that this triplet is a P.V. and let $H$ be its generic isotropy subgroup. Then we have $\operatorname{dim} H=\operatorname{dim} \operatorname{Spin}(9) \times G L(2)-\operatorname{dim} V(16) \otimes V(2)$ $=8$. This implies that for a suitable $x_{0}$ in $V(10)$, the isotropy subalgebra $\mathfrak{g}_{x_{0}}=\left\{A \in \mathfrak{g} \subset \mathfrak{D}(10) \mid A x_{0}=0\right\}$ of $\mathfrak{g} \cong\left(\mathfrak{g}_{2}\right) \oplus \mathfrak{g l}(2)$ which consists of $10 \times 10$ matrices $A$ in (5.40), is of dimension eight. Therefore there exists a 9dimensional orbit of $\mathfrak{g}^{\prime}$ which consists of $10 \times 10$ matrices in (5.42), since $\operatorname{dim} \mathrm{g}^{\prime}-8=9$. However, it is impossible since there exist algebraically independent two quadratic forms $f_{1}(x)$ and $f_{2}(x)$ where $f_{1}(x)=f_{1}\left(x_{1}\right.$, $\left.x_{2}, x_{3}\right)$ is absolutely invariant under the action of $\mathfrak{g l}(2)$ and $f_{2}(x)=f_{2}\left(x_{4}\right.$, $\left.\cdots, x_{10}\right)$ is absolutely invariant under the action of $\left(\mathfrak{g}_{2}\right)$ (See Proposition 25 or (32)). Hence it is not a P.V.

Proposition 36. A triplet $\left(\operatorname{Spin}(9) \times G L(2)\right.$, spin rep. $\otimes \Lambda_{1}, V(16) \otimes$ $V(2))$ is not a P.V.

$$
\left(\operatorname{Spin}(12) \times G L(d), \text { half-spin rep. } \otimes \Lambda_{1}, V(32) \otimes V(d)\right) \quad(1 \leq d \leq 2)
$$

I) The case of $d=1$.

The representation space $V=V(1) \otimes V(32)$ is spanned by $1, e_{i} e_{j}$, $e_{k} e_{\ell} e_{m} e_{n}, e_{1} e_{2} e_{3} e_{4} e_{5} e_{6}(1 \leq i<j \leq 6,1 \leq k<\ell<m<n \leq 6)$. Put $X_{0}=1$ 
$+e_{1} e_{2} e_{3} e_{4} e_{5} e_{6}$. We shall calculate the isotropy subalgebra $g_{X_{0}}$ of $g=g r(1)$ $\oplus \mathfrak{o}(12)$ at $X_{0}$. We may assume that an element $A$ of $\mathfrak{o}(12)$ is of the form (5.28).

Then by (5.29), we have $d \rho_{1}(A) \cdot 1=-\frac{1}{2}\left(a_{1}+\cdots+a_{6}\right)+\sum_{i<j} b_{i j} e_{i} e_{j}$ and $d \rho_{1}(A) e_{1} e_{2} e_{3} e_{4} e_{5} e_{6}=\frac{1}{2}\left(a_{1}+\cdots+a_{6}\right) e_{1} e_{2} e_{3} e_{4} e_{5} e_{6}+\sum_{i<j} c_{i j} \delta_{f i} \delta_{f j} e_{1} e_{2} e_{3} e_{4} e_{5} e_{6}$ and hence $a X_{0}+d \rho_{1}(A) X_{0}=\left(a-\frac{1}{2}\left(a_{1}+\cdots+a_{6}\right)\right)+\sum_{i<j} b_{i j} e_{i} e_{j}+\sum_{i<j} c_{i j}$ $\delta_{f_{i}} \delta_{f_{j}} e_{1} e_{2} e_{3} e_{4} e_{5} e_{6}+\left(a+\frac{1}{2}\left(a_{1}+\cdots+a_{6}\right)\right) e_{1} e_{2} e_{3} e_{4} e_{5} e_{6}$ for $(a, A) \in \mathfrak{g l}(1) \oplus \mathfrak{D}$ (12). Hence the isotropy subalgebra $\mathrm{g}_{X_{0}}=\left\{(a, A) \mid a X_{0}+d \rho_{1}(A) X_{0}=0\right\}$ is given by

$$
\mathfrak{g}_{X_{0}}=\left\{(0) \oplus\left[\frac{A}{0} \mid \frac{0}{-{ }^{t} A}\right] \mid A \in \mathfrak{B l}(6)\right\} \cong \mathscr{g l}(6) .
$$

Since $\operatorname{dim} g_{X_{0}}=35=\operatorname{dim} G L(1) \times S p i n(12)-\operatorname{dim} V(1) \otimes V(32)$, it is a P.V. and by Proposition 25 in $\S 4$, it is regular. This space was investigated by J. Igusa and the explicit form of the relative invariant quartic form $f(x)$ is given as follows (See [2]).

$$
\begin{aligned}
f(x)= & x_{0} \operatorname{Pff}\left(\left(y_{i j}\right)\right)+y_{0} \operatorname{Pff}\left(\left(x_{i j}\right)\right)+\sum_{i<j} \operatorname{Pff}\left(X_{i j}\right) \operatorname{Pff}\left(Y_{i j}\right) \\
& -\frac{1}{4}\left(x_{0} y_{0}-\sum_{i<j} x_{i j} y_{i j}\right)^{2}
\end{aligned}
$$

for $x=x_{0}+\sum_{i<j} x_{i j} e_{i} e_{j}+\sum_{i<j} y_{i j} e_{i j}^{*}+y_{0} e_{1} e_{2} e_{3} e_{4} e_{5} e_{6}$ in which, e.g., $\left(x_{i j}\right)$ is the alternating matrix determined by $x_{i j}$ and $X_{i j}$ the alternating matrix obtained from $\left(x_{i j}\right)$ by crossing out its $i$-th and $j$-th lines and columns, and $e_{i j}^{*}=(-1)^{i+j-1} e_{1} \cdots e_{i-1} e_{i+1} \cdots e_{j-1} e_{j+1} \cdots e_{6}$.

Proposition 37. A triplet $(G L(1) \times$ Spin(12), $\square \otimes$ half-spin rep., $V(1) \otimes V(32))$ is a regular P.V. and its generic isotropy subgroup is locally isomorphic to $S L(6)$. The relative invariant is a quartic form.

II) The case of $d=2$.

We identify $V=V(32) \otimes V(2)$ with $V(32) \oplus V(32)$. Assume that this triplet is a P.V. and let $X_{0}=\left(x_{0}^{\prime}, x_{0}^{\prime \prime}\right)$ is a generic point. Then the isotropy subgroup $H$ at $X_{0}$ is of dimension 6 (= $\operatorname{dim} \operatorname{Spin}(12) \times G L(2)-$ $\operatorname{dim} V(32) \otimes V(2))$. From ths case of $d=1$, we may assume that $x_{0}^{\prime}=1$ $+e_{1} e_{2} e_{3} e_{4} e_{5} e_{6}$. As a representation space of the isotropy subgroup $S L(6)$ at $x_{0}^{\prime}, V(32)$ decomposes into $V(32)=V_{1}(1) \oplus V_{2}(1) \oplus V_{3}(15) \oplus V_{4}(15)$ i.e. $1 \oplus 1 \oplus \Lambda_{2} \oplus \Lambda_{4}$ since the weights $\frac{1}{2}\left( \pm a_{1} \pm a_{2} \cdots \pm a_{6}\right)$ of $\mathfrak{g l}(6)$ where $a_{1}+$ $\cdots+a_{6}=0$ and the number of the signature + is even, decomposes to $\{0\} \cup\{0\} \cup\left\{a_{i}+a_{j} \mid 1 \leq i<j \leq 6\right\} \cup\left\{-a_{i}-a_{j} \mid 1 \leq i<j \leq 6\right\}$. Note that $\Lambda_{4}$ 
is the contragredient representation of $\Lambda_{2}$. We may assume that $x_{0} \in V_{1}(1)$ and $x_{0}^{\prime}=x_{2}^{\prime}+x_{3}^{\prime}+x_{4}^{\prime}$ with $x_{2}^{\prime} \in V_{2}(1), x_{3}^{\prime} \in V_{3}(15), x_{4}^{\prime} \in V_{4}(15)$. Then together with scalar multiplications, $S L(2)$ acts on $V_{3}(15)$ prehomogeneously and we may assume that $x_{3}^{\prime}$ is its generic point. Then the isotropy subgroup at $x_{3}^{\prime}$ is $S p(3)$ by Proposition 4. As a representation space of $S p(3)$, $V_{4}(15)$ decomposes to $V_{4}(15)=V_{5}(1) \oplus V_{6}(14)$ i.e. $1 \oplus \Lambda_{2}$, since $\left\{-a_{i}-a_{j} \mid 1\right.$ $\leq i<j \leq 6\}$ with $a_{4}=-a_{1}, a_{5}=-a_{2}, a_{6}=-a_{3}$ decomposes to $\{0\} \cup\{0,0$, $\left.\pm a_{i} \pm a_{j}, i<j=1,2,3\right\}$. An element of $V_{6}(14)$ is generically transferred to the form $\lambda_{1} u_{1} \wedge u_{4}+\lambda_{2} u_{2} \wedge u_{5}+\lambda_{3} u_{3} \wedge u_{6}$ with $\lambda_{1}+\lambda_{2}+\lambda_{3}=0$, under the action of $S p(3)$, and the isotropy subgroup at this point contains $S L(2) \times$ $S L(2) \times S L(2)$. This implies that the generic isotropy subgroup $H$ at $X_{0}$ $=\left(x_{0}^{\prime}, x_{0}^{\prime \prime}\right)$ contains $S L(2) \times S L(2) \times S L(2)$ and hence $6=\operatorname{dim} H \geqq \operatorname{dim} S L(2)$ $\times S L(2) \times S L(2)=9$, i.e., a contradiction. Therefore it is not a P.V.

Proposition 38. A triplet $\left(\operatorname{Spin}(12) \times G L(2)\right.$, half-spin rep. $\otimes \Lambda_{1}$, $V(32) \otimes V(2))$ is not a P.V.

$$
(G L(1) \times \operatorname{Spin}(11), \square \otimes \operatorname{spin} \text { rep., } \quad V(1) \otimes V(32)) .
$$

Put $v_{1}=e_{6}-f_{6}={ }^{t}(00000100000-1) \in V(12)$. Then we have $\mathfrak{o}(11, C)$ $=\left\{A \in \mathfrak{D}(12, C) \mid A v_{1}=0\right\}$ and hence an element $A$ of the form (5.28) is in $\mathfrak{o}(11, C)$ if and only if $a_{6}=0, a_{i 6}=b_{i 6}$ and $a_{6 i}=-c_{i 6}$ for $1 \leq i \leq 5$. Therefore the isotropy subalgebra $\mathfrak{g}_{X_{0}}$ of $\mathfrak{g}=\mathfrak{g r}(1) \oplus \mathfrak{o}(11)$ at $X_{0}=1+e_{1} e_{2} e_{3} e_{4} e_{5} e_{6}$ is given by

$$
\mathfrak{g}_{X_{0}}=\mathfrak{o}(11) \cap\left\{\left(\frac{A^{\prime}}{\mid-{ }^{t} A^{\prime}}\right) \mid A^{\prime} \in \mathfrak{g l}(6)\right\}=\left\{\left(\frac{A^{\prime}}{\mid-{ }^{t} A^{\prime}}\right) \mid A^{\prime} \in \mathfrak{g l}(5)\right\} \cong \mathfrak{g l}(5)
$$

by Proposition 37. Since $\operatorname{dim} g_{X_{0}}=24=\operatorname{dim} G L(1) \times \operatorname{Spin}(11)-\operatorname{dim} V(1)$ $\otimes V(32)$, this triplet is a P.V. and it is regular by Proposition 25 in $\S 4$. The relative invariant is the quartic form in (5.48). This space was investigated by J. Igusa (See [2]).

Proposition 39. A triplet $(G L(1) \times \operatorname{Spin}(11), \square \otimes \operatorname{spin}$ rep., $V(1) \otimes$ $V(32)$ ) is a regular P.V. and its generic isotropy subgroup is locally isomorphic to $S L(5)$. The relative invariant is the same quartic form as in Proposition 37.

$$
(G L(1) \times \operatorname{Spin}(14), \square \otimes h a l f-s p i n \text { rep., } V(1) \otimes V(64))
$$

The representation space $V=V(1) \otimes V(64)$ is spanned by $1, e_{i} e_{j}(1 \leq$ 
$i<j \leq 7), e_{i} e_{j} e_{k} e_{\ell}(1 \leq i<j<k<\ell \leq 7)$ and $e_{i} e_{j} e_{k} e_{\ell} e_{m} e_{n}(1 \leq i<j<k$ $<\ell<m<n \leq 7)$. Put $X_{0}=1+e_{1} e_{2} e_{3} e_{7}+e_{4} e_{5} e_{6} e_{7}+e_{1} e_{2} e_{3} e_{4} e_{5} e_{6}$. We shall calculate the isotropy subalgebra $\mathfrak{g}_{X_{0}}$ of $\mathfrak{g}=\mathfrak{g r}(1) \oplus \mathfrak{o}(14)$ at $X_{0}$. We may assume that an element $A$ of $\mathfrak{o}(14, C)$ is of the form (5.28). Let $d \rho_{1}$ be the half-spin representation of $\mathfrak{o}(14, C)$. Then by (5.29) we have

$$
\begin{aligned}
d \rho_{1}(A) \cdot 1= & -\frac{1}{2}\left(a_{1}+\cdots+a_{7}\right)+\sum_{i<j} b_{i j} e_{i} e_{j}, \\
d \rho_{1}(A) e_{1} e_{2} e_{3} e_{7}= & \sum_{i=4}^{6} a_{i 1} e_{2} e_{3} e_{i} e_{7}-\sum_{i=4}^{6} a_{i 2} e_{1} e_{3} e_{i} e_{7}+\sum_{i=4}^{6} a_{i 3} e_{1} e_{2} e_{i} e_{7}+\sum_{i=4}^{6} a_{i 7} e_{1} e_{2} e_{3} e_{i} \\
& +\frac{1}{2}\left(a_{1}+a_{2}+a_{3}-a_{4}-a_{5}-a_{6}+a_{7}\right) e_{1} e_{2} e_{3} e_{7} \\
& +\sum_{4 \leq i<j \leq 6} e_{1} e_{2} e_{3} e_{i} e_{j} e_{7}-c_{17} e_{2} e_{3}+c_{27} e_{1} e_{3}-c_{37} e_{1} e_{2} \\
& -\left(c_{23} e_{1}-c_{13} e_{2}+c_{12} e_{3}\right) e_{7},
\end{aligned}
$$

and

$$
\begin{aligned}
d \rho_{1}(A) e_{1} e_{2} \cdots e_{6}= & \sum_{j=1}^{6}(-1)^{j-1} a_{7 j} e_{1} \cdots e_{j-1} e_{j+1} \cdots e_{7} \\
& +\frac{1}{2}\left(a_{1}+\cdots+a_{6}-a_{7}\right) e_{1} e_{2} \cdots e_{6} \\
& +\sum_{i<j \leq 6}(-1)^{i+j} e_{1} \cdots e_{i-1} e_{i+1} \cdots e_{j-1} e_{j+1} \cdots e_{6} .
\end{aligned}
$$

By changing indices from $(1, \ldots, 7)$ to $(4,5,6,1,2,3,7)$, we obtain $d \rho_{1}(A)$ $e_{4} e_{5} e_{6} e_{7}$ from $d \rho_{1}(A) e_{1} e_{2} e_{3} e_{7}$. Hence the isotropy subalgebra $g_{X_{0}}=\{(a, A) \in$ $\left.\mathrm{gl}(1) \oplus \mathfrak{o}(14) \mid a X_{0}+d \rho_{1}(A) X_{0}=0\right\}$ is given by

$$
\begin{aligned}
\mathfrak{g}_{X_{0}} & =\left\{(0) \oplus\left(\frac{A_{1}}{A_{3}} \mid \frac{A_{2}}{-{ }^{t} A_{1}}\right) \in \mathfrak{g r}(1) \oplus \mathfrak{o}(14) \mid A_{1}, A_{2}, A_{3} \text { are of the form (5.49) }\right\} \\
& \cong\left\{\left(A_{4}\right) \oplus\left(A_{5}\right) \mid A_{4}, A_{5} \text { are of the form }(5.49)\right\} \cong\left(\mathrm{g}_{2}\right) \oplus\left(\mathrm{g}_{2}\right)
\end{aligned}
$$

(See $(1.8)$ in $\S 1)$.

$$
A_{1}=\left(\begin{array}{c|c|c}
X & 0 & a \\
b \\
c \\
0 & Y & \frac{d}{e} \\
\hdashline u v w & \lambda \mu \nu & 0
\end{array}\right) \quad \text { with } X, Y \in \operatorname{gr}(3)
$$




$$
\begin{aligned}
& A_{2}=\left(\begin{array}{rrr|rrr|r}
0 & w & -v & & & & -a \\
-w & 0 & u & & 0 & & -b \\
v & -u & 0 & & & & -c \\
\hline & & & 0 & -\nu & \mu & d \\
& 0 & & \nu & 0 & -\lambda & e \\
& & & -\mu & \lambda & 0 & f \\
\hline a & b & c & -d & -e & -f & 0
\end{array}\right), \\
& A_{3}=\left(\begin{array}{rrr|rrr|r}
0 & -c & b & & & & u \\
c & 0 & -a & & 0 & & v \\
-b & a & 0 & & & & w \\
\hline & & & 0 & f & -e & -\lambda \\
& 0 & & -f & 0 & d & -\mu \\
& & & e & -d & 0 & -\nu \\
\hline-u & -v & -w & \lambda & \mu & \nu & 0
\end{array}\right), \\
& A_{4}=\left(\begin{array}{c|rrr|rrr}
0 & 2 u & 2 v & 2 w & 2 a & 2 b & 2 c \\
\hline a & & & & 0 & w & -v \\
b & & X & & -w & 0 & u \\
c & & & & v & -u & 0 \\
\hline u & 0 & -c & b & & & \\
v & c & 0 & -a & & -{ }^{t} X \\
w & -b & a & 0 & &
\end{array}\right], \\
& A_{5}=\left(\begin{array}{c|rrr|rrr}
0 & 2 \lambda & 2 \mu & 2 \nu & 2 d & 2 e & 2 f \\
\hline d & & & & 0 & \nu & -\mu \\
e & & Y & & -\nu & 0 & \lambda \\
f & & & & \mu & -\lambda & 0 \\
\hline \lambda & 0 & -f & e & & & \\
\mu & f & 0 & -d & & -{ }^{t} Y & \\
\nu & -e & d & 0 & & &
\end{array}\right] .
\end{aligned}
$$

Since $\operatorname{dim} g_{X_{0}}=28=\operatorname{dim} G L(1) \times \operatorname{Spin}((14)-\operatorname{dim} V(1) \otimes V(64)$, this triplet is a P.V. and it is regular by Proposition 25 in $\S 4$. The prehomogeneity of this space was proved by T. Shintani (1970) and the orbital decomposition was completed by I. Ozeki and the second author (1973) (See [12]). Put $X_{0}^{\prime}=1+e_{1} e_{2} e_{3} e_{7}+e_{1} e_{4} e_{5} e_{7}+e_{2} e_{4} e_{6} e_{7}+e_{1} e_{2} e_{3} e_{4} e_{5} e_{6}$. Then by the similar calculation, the isotropy subalgebra $g_{X_{0}^{\prime}}$ at $X_{0}^{\prime}$ is given by

$$
\mathrm{g}_{X_{0}^{\prime}}=\left\{\left(\frac{a_{7}}{2}\right) \oplus\left[\frac{A_{1}^{\prime}}{A_{3}^{\prime}} \mid \frac{A_{2}^{\prime}}{-{ }^{t} A_{1}^{\prime}}\right] \mid A_{1}^{\prime}, A_{2}^{\prime}, A_{3}^{\prime} \text { are given in (5.50) }\right\}
$$




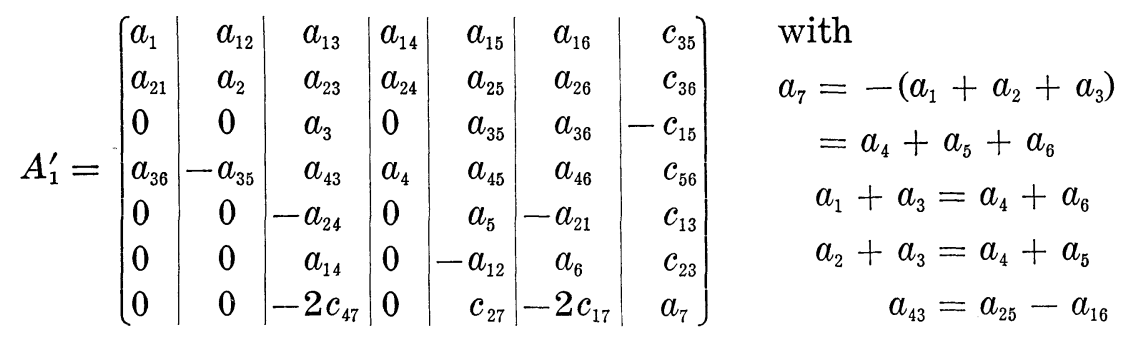

$$
\begin{aligned}
& A_{2}^{\prime}=\left(\begin{array}{c|c|c|c|c|c|c}
0 & c_{37} & -c_{27} & c_{57} & -c_{47} & 0 & 2 c_{23} \\
-c_{37} & 0 & c_{17} & c_{67} & 0 & -c_{47} & -2 c_{13} \\
c_{27} & -c_{17} & 0 & 0 & 0 & 0 & 0 \\
-c_{57} & -c_{67} & 0 & 0 & c_{17} & c_{27} & -2 c_{15} \\
c_{47} & 0 & 0 & -c_{17} & 0 & 0 & 0 \\
0 & c_{47} & 0 & -c_{27} & 0 & 0 & 0 \\
-2 c_{23} & 2 c_{13} & 0 & 2 c_{15} & 0 & 0 & 0
\end{array}\right) \\
& A_{3}^{\prime}=\left(\begin{array}{c|c|c|c|c|c|c}
0 & 0 & c_{13} & 0 & c_{15} & 0 & c_{17} \\
0 & 0 & c_{23} & 0 & 0 & c_{15} & c_{27} \\
-c_{13} & -c_{23} & 0 & 0 & c_{35} & c_{36} & c_{37} \\
0 & 0 & 0 & 0 & c_{23} & -c_{13} & c_{47} \\
-c_{15} & 0 & -c_{35} & -c_{23} & 0 & c_{56} & c_{57} \\
0 & -c_{15} & -c_{36} & c_{13} & -c_{56} & 0 & c_{67} \\
-c_{17} & -c_{27} & -c_{37} & -c_{47} & -c_{57} & -c_{67} & 0
\end{array}\right) \text {. }
\end{aligned}
$$

Since $\operatorname{dim} g_{X_{0}^{\prime}}=29$, the orbit of $X_{0}^{\prime}$ is of codimension one. Since the trace $\operatorname{tr}_{V} A$ of an element $A$ of $\mathfrak{g}_{X_{0}^{\prime}}$ on $V$ is $64 \times\left(a_{7} / 2\right)=32 a_{7}$ and the adjoint representation on $g_{X_{0}^{\prime}}$ is $-28 a_{7}$, the degree of the irreducible relative invariant polynomial $f(x)$ is given by $\operatorname{deg} f=\left(32 a_{7}-28 a_{7}\right) / 32 a_{7}$ $\times 64=8$.

Proposition 40. A triplet $(G L(1) \times \operatorname{Spin}(14), \square \otimes$ half-spin rep., $V(1)$ $\otimes V(64))$ is a regular P.V. and its generic isotropy subgroup is locally isomorphic to $\left(G_{2}\right) \times\left(G_{2}\right)$. The relative invariant is of degree 8 .

$$
(G L(1) \times S p i n(13), \square \otimes \text { spin rep., } V(1) \otimes V(64)) .
$$

This space was investigated by T. Shintani (1971). We shall prove the non-prehomogeneity of this triplet after T. Shintani.

Assume that this triplet is a P.V. Let $h$ be the generic isotropy subalgebra of the regular P.V. in Proposition 40. Then there exists a 14-dimensional vector $x_{0}$ such that $\mathfrak{h} \cap\left(\mathfrak{g l}(1) \oplus\left\{A \in \mathfrak{D}(14) \mid A x_{0}=0\right\}\right)=$ $\left\{A \in \mathfrak{h} \mid A x_{0}=0\right\}$ is of dimension $15(=\operatorname{dim} G L(1) \times \operatorname{Spin}(13)-\operatorname{dim} V(1) \otimes$ $V(64))$. By Proposition $40, \mathfrak{h} \cong\left(\mathfrak{g}_{2}\right) \oplus\left(\mathfrak{g}_{2}\right)$ and $\mathfrak{h}$ is contained in $\mathfrak{b}(14)$ as 
the direct sum of 7-dimensional representations, this implies that the (non-irreducible) triplet $\left(G L(1) \times\left(G_{2}\right) \times\left(G_{2}\right), \square \otimes\left(\Lambda_{2} \otimes 1+1 \otimes \Lambda_{2}\right), \quad V(1)\right.$ $\otimes\left(V_{1}(7) \oplus V_{2}(7)\right)$ is a P.V. By Proposition 25, there exists a quadratic invariant $q_{i}(x)$ on $V(1) \otimes V_{i}(7)(i=1,2)$ and the quotient $q(x)=q_{1}(x) / q_{2}(x)$ is a non-constant absolute invariant of this triplet. By Proposition 3 in $\S 2$, it is not a P.V. and hence our triplet is not a P.V.

Proposition 41. A triplet $(G L(1) \times \operatorname{Spin}(13), \square \otimes \operatorname{spin}$ rep., $V(1) \otimes$ $V(64))$ is not a P.V.

$$
\left(\left(G_{2}\right) \times G L(d), \Lambda_{2} \otimes \Lambda_{1}, V(7) \otimes V(d)\right) \quad(1 \leq d \leq 3)
$$

I) The case of $d=1$.

Let $g$ be the Lie algebra of $G L(1) \times\left(G_{2}\right)$. Then by Example 30 in $\S 1$, we have

$$
\mathfrak{g}=\left\{A=\left(\begin{array}{c|ccc|ccc}
\xi & 2 d & 2 e & 2 f & 2 a & 2 b & 2 c \\
\hline a & \xi+\lambda_{1} & \lambda_{12} & \lambda_{13} & 0 & f & -e \\
b & \lambda_{21} & \xi+\lambda_{2} & \lambda_{23} & -f & 0 & d \\
c & \lambda_{31} & \lambda_{32} & \xi+\lambda_{3} & e & -d & 0 \\
\hline d & 0 & -c & b & \xi-\lambda_{1} & -\lambda_{21} & -\lambda_{31} \\
e & c & 0 & -a & -\lambda_{12} & \xi-\lambda_{2} & -\lambda_{32} \\
f & -b & a & 0 & -\lambda_{13} & -\lambda_{23} & \xi-\lambda_{3}
\end{array}\right) \mid \lambda_{1}+\lambda_{2}+\lambda_{3}=0\right\}
$$

Put $X_{0}={ }^{t}(1,0,0,0,0,0,0)$. Then $A X_{0}={ }^{t}(\xi, a, b, c, d, e, f)$. Therefore the isotropy subalgebra $\mathfrak{g}_{X_{0}}$ at $X_{0}$ is given by

$$
\begin{aligned}
\mathfrak{g}_{X_{0}} & =\left\{A \in \mathfrak{g} \mid A X_{0}=0\right\}=\{A \in \mathfrak{g} \mid \xi=a=b=c=d=e=f=0\} \\
& =\left\{\left(-\mid \frac{\Lambda}{\mid-{ }^{t} \Lambda}\right) \mid \Lambda \in \mathfrak{g l}(3, C)\right\} \cong \mathfrak{g}((3, C) .
\end{aligned}
$$

Since $\operatorname{dim} \mathfrak{g}_{X_{0}}=8=\operatorname{dim} \mathfrak{g}-\operatorname{dim} V$ and $g_{X_{0}}$ is reductive, it is a regular P.V. by Proposition 25 in $\S 4$. By Proposition 25 (in $\S 5$ ), there exists a relative invariant quadratic form, i.e., $\left(G_{2}\right) \subset S O(7)$.

We shall determine the relative invariant $f(x)=\sum_{1 \leq i \leq j \leq 7} c_{i j} x_{i} x_{j}$. Let $A$ be a diagonal matrix in (5.51). Then

$$
\begin{aligned}
\langle A x, \operatorname{grad} f(x)\rangle= & \sum_{j=1}^{7} \xi x_{1} \frac{\partial f}{\partial x_{j}}+\sum_{j=1}^{7}\left(\xi+\lambda_{1}\right) x_{2} \frac{\partial f}{\partial x_{j}}+\cdots \\
& +\sum_{j=1}^{7}\left(\xi-\lambda_{3}\right) x_{7} \frac{\partial f}{\partial x_{j}}=2 \xi f(x)
\end{aligned}
$$


and hence $f(x)=c_{11} x_{1}^{2}+c_{25} x_{2} x_{5}+c_{36} x_{3} x_{6}+c_{47} x_{4} x_{7}$. Let $A_{1}$ (resp. $A_{2}$ ) be the matrix with $a=1$ (resp. $b=1$ ), all remaining entries zero in (5.51). Then $\left\langle A_{1} x, \operatorname{grad} f(x)\right\rangle=\left(4 c_{11}+c_{25}\right) x_{1} x_{5}+\left(c_{47}-c_{36}\right) x_{3} x_{4}=0$ and hence $f(x)=$ $c_{11}\left(x_{1}^{2}-4 x_{2} x_{5}\right)+c_{36}\left(x_{3} x_{6}+x_{4} x_{7}\right)$. Since $\left\langle A_{2} x, \operatorname{grad} f(x)\right\rangle=c_{11}\left(4 x_{1} x_{6}-4 x_{2} x_{4}\right)$ $+c_{36}\left(x_{1} x_{6}-x_{2} x_{4}\right)=0$, we have $f(x)=c_{11}\left(x_{1}^{2}-4 x_{2} x_{5}-4 x_{3} x_{6}-4 x_{4} x_{7}\right)$.

Proposition 42. A triplet $\left(G L(1) \times\left(G_{2}\right), \square \otimes \Lambda_{2}, V(1) \otimes V(7)\right)$ is a regular $P . V$. and its generic isotropy subgroup is locally isomorphic to $S L(3)$. The relative invariant is a quadratic form.

II) The case of $d=2$.

If we identify $V(7) \otimes V(2)$ with $7 \times 2$ matrices $M(7,2)$, the action $d \rho$ of the Lie algebra $\mathfrak{g}=\left(\mathfrak{g}_{2}\right) \oplus \mathfrak{g r}(2)$ of $\left(G_{2}\right) \times G L(2)$ is given by $d \rho(A) X$ $=B X+X^{t} C$ where $X \in M(7,2), A=(B, C) \in \mathfrak{g}=\left(g_{2}\right) \oplus \mathfrak{g l}(2)$. Put

$$
X_{0}={ }^{t}\left(\begin{array}{l}
0100000 \\
0000100
\end{array}\right) \text {. }
$$

Then

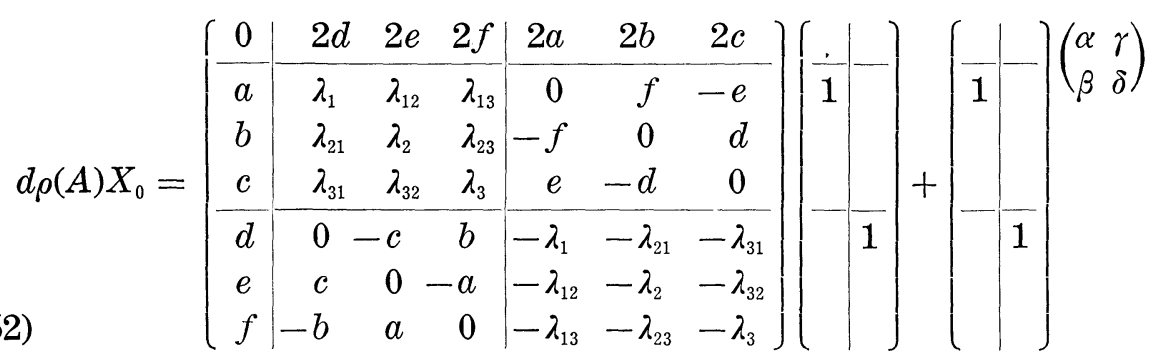

$$
\begin{aligned}
& =\left(\begin{array}{c|c}
2 d & 2 a \\
\hline \lambda_{1}+\alpha & \gamma \\
\lambda_{21} & -f \\
\lambda_{31} & e \\
\hline \beta & -\lambda_{1}+\delta \\
c & -\lambda_{12} \\
-b & -\lambda_{13}
\end{array}\right)
\end{aligned}
$$

Hence the isotropy subalgebra $\mathfrak{g}_{X_{0}}$ at $X_{0}$ is by definition $\mathfrak{g}_{X_{0}}=\left\{A \in \mathfrak{g} \mid d \rho(A) X_{0}\right.$ $=0\}$ and hence we have 
(5.53)

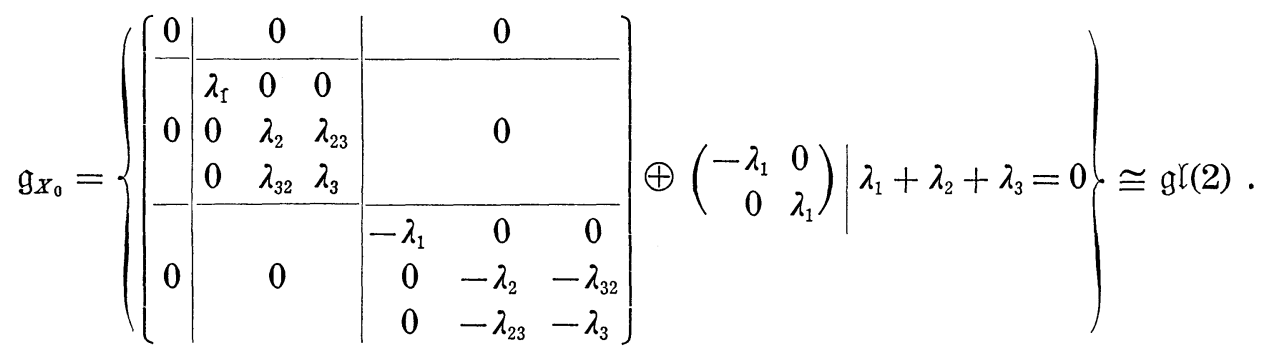

Since $\operatorname{dim} \mathfrak{g}_{X_{0}}=4=\operatorname{dim} \mathfrak{g}-\operatorname{dim} V$, it is a regular P.V. by Proposition 25 in $\S 4$. Since $\left(G_{2}\right) \times G L(2) \subset S O(7) \times G L(2)$, there exists an irreducible relative invariant polynomial of degree 4 (See (20)).

Proposition 43. A triplet $\left(\left(G_{2}\right) \times G L(2), \Lambda_{2} \otimes \Lambda_{1}, V(7) \otimes V(2)\right)$ is a regular $P . V$. and its generic isotropy subgroup is locally isomorphic to GL(2). The relative invariant is a quartic form.

III) The case of $d=3$.

Identify $V(7) \otimes V(3)$ with $7 \times 3$ matrices $M(7,3)$. Since $\left(G_{2}\right) \times G L(3)$ $\subset S O(7) \times G L(3)$, there exists an irreducible relative invariant polynomial $f_{1}(x)$ of degree 6 (See (20)). On the other hand, $\left(G_{2}\right)$ is the isotropy subgroup at $X_{0}=u_{2} \wedge u_{3} \wedge u_{4}+u_{5} \wedge u_{6} \wedge u_{7}+u_{1} \wedge\left(u_{2} \wedge u_{5}+u_{3} \wedge u_{6}+u_{4} \wedge u_{7}\right)$ (See (8)), and hence the group $\left(G_{2}\right) \times S L(3)$ leaves the following polynomial $f_{2}(x)$ invariant.

$$
\begin{aligned}
& f_{2}(X)=\operatorname{det}\left(\begin{array}{lll}
x_{2} & y_{2} & z_{2} \\
x_{3} & y_{3} & z_{3} \\
x_{4} & y_{4} & z_{4}
\end{array}\right)+\operatorname{det}\left(\begin{array}{lll}
x_{5} & y_{5} & z_{5} \\
x_{6} & y_{6} & z_{6} \\
x_{7} & y_{7} & z_{7}
\end{array}\right)+\operatorname{det}\left(\begin{array}{lll}
x_{1} & y_{1} & z_{1} \\
x_{2} & y_{2} & z_{2} \\
x_{5} & y_{5} & z_{5}
\end{array}\right)+\operatorname{det}\left(\begin{array}{lll}
x_{1} & y_{1} & z_{1} \\
x_{3} & y_{3} & z_{3} \\
x_{6} & y_{6} & z_{6}
\end{array}\right) \\
& +\operatorname{det}\left(\begin{array}{lll}
x_{1} & y_{1} & z_{1} \\
x_{4} & y_{4} & z_{4} \\
x_{7} & y_{7} & z_{7}
\end{array}\right) \quad \text { where } X=\left(\begin{array}{lll}
x_{1} & \cdots & x_{7} \\
y_{1} & \cdots & y_{7} \\
z_{1} & \cdots & z_{7}
\end{array}\right) \in V(7) \otimes V(3)
\end{aligned}
$$

Since the quotient $f_{1}(x) / f_{2}(x)^{2}$ is a non-constant absolute invariant, it is not a P.V. by Proposition 3 in $\S 2$ (or Proposition 12 in $\S 4$ ).

Proposition 44. A triplet $\left(\left(G_{2}\right) \times G L(3), \Lambda_{2} \otimes \Lambda_{1}, V(7) \otimes V(3)\right)$ is not a P.V. There exist two irreducible relative invariant of degree 3 and degree 6.

$$
\left(F_{4} \times G L(d), \Lambda_{4} \otimes \Lambda_{1}, V(26) \otimes V(d)\right) \quad(1 \leq d \leq 2)
$$


I) The case of $d=1$.

By Proposition 39 in $\S 1, f_{1}(X)=\operatorname{Tr} X \circ X$ and $f_{2}(X)=\operatorname{Tr} X \circ X \circ X$ $\left(x \in \mathscr{J}_{0}\right)$ is a relative invariant of $G L(1) \times F_{4}$, and hence $f(x)=f_{2}(x)^{2} \cdot f_{1}(x)^{-3}$ is an absolute invariant. Since

$$
f\left(\left(\begin{array}{ccc}
1 & & \\
& 0 & \\
& & -1
\end{array}\right)\right)=0 \text { and } f\left(\left(\begin{array}{lll}
1 & & \\
& -2 & \\
& & 1
\end{array}\right)\right) \neq 0
$$

$f(x)$ is not constant, and hence it is not a P.V. by Proposition 3 in $\S 2$.

Proposition 45. A triplet $\left(G L(1) \times F_{4}, \square \otimes \Lambda_{4}, V(1) \otimes V(26)\right)$ is not a P.V. There exist two irreducible relative invariants of degree 2 and degree 3.

II) The case of $d=2$.

Identify $V(26) \otimes V(2)$ with $\mathscr{J}_{0} \oplus \mathscr{J}_{0}$ and put $f_{1}(X)=\operatorname{Tr} X \circ X, f_{2}(X)$ $=\operatorname{Tr} X \circ X \circ X$ for $X \in \mathscr{J}_{0} . \quad$ For $X=\left(X_{1}, X_{2}\right) \in \mathscr{J}_{0} \oplus \mathscr{J}_{0}$, the polynomial $f_{1}\left(u X_{1}+v X_{2}\right)$ is a binary quadratic form which is invariant under the action of $F_{4}$. Therefore, its discriminant $g_{1}(X)$ is a relative invariant of $F_{4} \times G L(2)$ (See (3)). Similarly the discriminant $g_{2}(X)$ of the binary cubic form $f_{2}\left(u X_{1}+v X_{2}\right)$ is also a relative invariant of $F_{4} \times G L(2)$ (See (6)). As $\operatorname{deg} g_{1}=4$ and $\operatorname{deg} g_{2}=12, f(x)=g_{2}(x) g_{1}(x)^{-3}$ for $x \in \mathscr{J}_{0} \oplus \mathscr{J}_{0}$ is an absolute invariant of $F_{4} \times G L(2)$. Since

$$
f\left(\left(\begin{array}{ccc}
1 & & \\
& 0 & \\
& & -1
\end{array}\right)\left(\begin{array}{lll}
0 & 1 & \\
1 & 0 & \\
& & 0
\end{array}\right)\right)=0 \text { and } f\left(\left(\begin{array}{ccc}
1 & & \\
& 0 & \\
& & -1
\end{array}\right)\left(\begin{array}{ccc}
0 & & \\
& 1 & \\
& & -1
\end{array}\right)\right) \neq 0,
$$

$f(x)$ is not constant, and hence it is not a P.V. by Proposition 3 in $\S 2$.

Proposition 46. A triplet $\left(F_{4} \times G L(2), \Lambda_{4} \otimes \Lambda_{1}, V(26) \otimes V(2)\right)$ is not a P.V.

$$
\left(E_{6} \times G L(d), \Lambda_{1} \otimes \Lambda_{1}, V(27) \otimes V(d)\right) \quad(1 \leq d \leq 3)
$$

I) The case of $d=1$.

Identify $V(1) \otimes V(27)$ with the exceptional simple Jordan algebra $\mathscr{J}$. The Lie algebra $\mathrm{g}$ of $E_{6} \times G L(1)$ is $\mathfrak{g}=\left\{A=D+R_{Y}+\alpha \mid D \in \mathscr{D}, Y \in \mathscr{J}_{0}\right.$, $\alpha \in C$ \} (See Example 39 in $\S 1$ ). Hence an element $A=D+R_{Y}+\alpha$ of $g$ is contained in the isotropy subalgebra $g_{X_{0}}$ at $X_{0}=\left(\begin{array}{lll}1 & & \\ & 1 & 1\end{array}\right) \in \mathscr{J}$ if and 


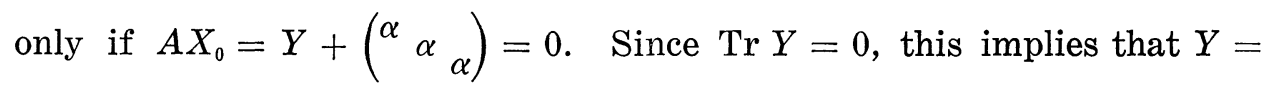
0 and $\alpha=0$. Therefore the isotropy subalgebra $g_{X_{0}}$ is the exceptional simple algebra $\mathscr{D} \cong F_{4}$. Since $\operatorname{dim} E_{6} \times G L(1)-\operatorname{dim} F_{4}=27=\operatorname{dim} \mathscr{J}$, it is a regular P.V. by Proposition 25 in $\S 4$. The relative invariant is the determinant $N(X)=\operatorname{det} X(X \in \mathscr{J})$ (See Example 39 in $\S 1$ ).

Proposition 47. A triplet $\left(G L(1) \times E_{6}, \square \otimes \Lambda_{1}, V(1) \otimes V(27)\right)$ is a regular P.V. and its generic isotropy subgroup is locally isomorphic to $F_{4}$. The relative invariant is the determinant of the exceptional simple Jordan algebra $\mathscr{J}(\cong V(1) \otimes V(27))$.

II) The case of $d=2$.

Identify $V(27) \otimes V(2)$ with $\mathscr{J} \oplus \mathscr{J}$. Let $\mathfrak{g}_{X_{0}}$ be the isotropy subalgebra of $E_{6} \oplus \operatorname{gr}(2)$ at $X_{0}=\left(\left(\begin{array}{lll}l_{1} & & \\ & & 1\end{array}\right),\left(\begin{array}{lll}{ }^{1} & 0 & \\ & & -1\end{array}\right)\right)$. Then it is obvious that $\mathscr{D}_{0} \subseteq \mathfrak{g}_{X_{0}}$ (See Definition 34 in $\S 1$ ). We shall show that $\mathscr{D}_{0} \supseteq \mathfrak{g}_{X_{0}}$. Let $z=X+\left(\begin{array}{ll}a & b \\ c & d\end{array}\right)$ be an element of $\mathrm{g}_{X_{0}}$, where $X=D+(\alpha)_{1}^{\prime}+(\beta)_{2}^{\prime}+$ $(\gamma)_{3}^{\prime}+R_{Y}\left(D \in \mathscr{D}_{0}, \operatorname{Tr} Y=0\right.$, See Definition 36 in $\left.\S 1\right)$. Then

$z \cdot X_{0}=\left(Y+\left(\begin{array}{ccc}a+b & & \\ & a & \\ & & a-b\end{array}\right), X\left(\begin{array}{ccc}1 & & \\ & 0 & \\ & & -1\end{array}\right)+\left(\begin{array}{ccc}c+d & & \\ & c & \\ & & c-d\end{array}\right)\right)=0$ implies that $Y+\left(\begin{array}{lll}a+b & a & \\ & a-b\end{array}\right)=0$. Since $\operatorname{Tr} Y=0$, we obtain that $Y=\left(\begin{array}{lll}b & 0 & \\ & & -b\end{array}\right)$ and $a=0$. Since $D\left(\begin{array}{lll}1 & 0 & \\ & & -1\end{array}\right)=0$ for $D \in \mathscr{D}_{0}$, we obtain that

$$
\begin{gathered}
X\left(\begin{array}{lll}
1 & & \\
& 0 & \\
& & -1
\end{array}\right)+\left(\begin{array}{ccc}
c+d & & \\
& c & \\
& & c-d
\end{array}\right) \\
+\left[\begin{array}{ccc}
b+c+d & -\frac{\gamma}{4} & -\frac{\beta}{2} \\
-\frac{\bar{\gamma}}{4} & c & -\frac{\alpha}{4} \\
-\frac{\beta}{2} & -\frac{\bar{\alpha}}{4} & b+c-d
\end{array}\right)=0 .
\end{gathered}
$$

Together with $Y=\left(\begin{array}{lll}-b & & \\ & 0 & b\end{array}\right)$ and $a=0$, we have $z=D \in \mathscr{D}_{0}$. Therefore 
we obtain that $\mathfrak{g}_{X_{0}} \cong \mathscr{D}_{0} \cong \mathfrak{v}(8, C)$ (See Proposition 35 in $\S 1$ ). Since $\operatorname{dim} g_{X_{0}}=28=\operatorname{dim} E_{6} \times G L(1)-\operatorname{dim} \mathscr{J} \oplus \mathscr{J}$, it is a regular P.V. by Proposition 25 in $\S 4$. For $X=\left(X_{1}, X_{2}\right) \in \mathscr{J} \oplus \mathscr{J}$, the discriminant $f(X)$ of the binary cubic form $N\left(u X_{1}+v X_{2}\right)$ is a relative invariant of degree 12. This is irreducible since the restriction of $f(X)$ to $\left\{\left(X_{1}, X_{2}\right) \in \mathscr{J} \oplus\right.$ $\left.\mathscr{J} \mid X_{1}, X_{2} \in M(3, C),{ }^{t} X_{1}=X_{1},{ }^{t} X_{2}=X_{2}\right\}$ is irreducible (See (10)).

Proposition 48. A triplet $\left(E_{6} \times G L(2), \Lambda_{1} \otimes \Lambda_{1}, V(27) \otimes V(2)\right)$ is a regular $P . V$. and its generic isotropy subgroup is locally isomorphic to the orthogonal group $O(8, C)$. The relative invariant is of degree 12.

Remark 49. The following four P.V.'s correspond to $\boldsymbol{R}, \boldsymbol{C}, \boldsymbol{H}$, 厄 respectively.

1) $\left(S L(3) \times G L(2), 2 \Lambda_{1} \otimes \Lambda_{1}, V(6) \otimes V(2)\right)$;

2) $\left(S L(3) \times S L(3) \times G L(2), \Lambda_{1} \otimes \Lambda_{1} \otimes \Lambda_{1}, V(3) \otimes V(3) \otimes V(2)\right)$;

3) $\left(S L(6) \times G L(2), \Lambda_{2} \otimes \Lambda_{1}, V(15) \otimes V(2)\right)$;

4) $\left(E_{6} \times G L(2), \Lambda_{1} \otimes \Lambda_{1}, V(27) \otimes V(2)\right)$.

Their representation spaces can be regarded as the pair of $3 \times 3$ hermitian matrices over some algebra $A$, where $A=C\left(=R \otimes_{R} C\right), C \oplus C$ (=C $\left.\otimes_{\boldsymbol{R}} \boldsymbol{C}\right), M_{2}(\boldsymbol{C})\left(=\boldsymbol{H} \otimes_{\boldsymbol{R}} \boldsymbol{C}\right)$ and $\mathbb{E}$ respectively. The rank $m$ of $A$ is $m=2^{k}(k=0,1,2,3)$, and the dimension of a generic isotropy group is given by $(k+1)(m-1)(k=0,1,2,3)$. The degree of the irreducible relative invariant is 12 .

III) The case of $d=3$.

We may identify $V=V(27) \otimes V(3)$ with $\mathscr{J} \oplus \mathscr{J} \oplus \mathscr{J}$ where $\mathscr{J}$ is the exceptional simple Jordan algebra.

Let $N\left(X_{1}\right)$ be the determinant of $X_{1}$ in $\mathscr{J}$ (See Example 39 in $\S 1$ ). For each $X=\left(X_{1}, X_{2}, X_{3}\right) \in V$, we can define the ternary cubic form $\varphi(X)$ $=N\left(u X_{1}+v X_{2}+w X_{3}\right)$ which is invariant under the action of $E_{6}$. This $\varphi$ is an equivariant map from $\mathscr{J} \oplus \mathscr{J} \oplus \mathscr{J}$ to the space of ternary cubic forms. Since a triplet $\left(E_{6} \times G L(3), 1 \otimes 3 \Lambda_{1}, V(10)\right) \cong\left(G L(3), 3 \Lambda_{1}, V(10)\right)$ is clearly not a P.V., if $\varphi$ is generically surjective, a triplet $\left(E_{6} \times G L(3)\right.$, $\left.\Lambda_{1} \otimes \Lambda_{1}, V(27) \otimes V(3)\right)$ is not a P.V. by Lemma 5 in $\S 2$. For $t \in C$, put

$$
X(t)=\left(\left(\begin{array}{rrr}
1 & & \\
& 0 & \\
& & -1
\end{array}\right)\left(\begin{array}{rrrr}
1 & & \\
& -1 & \\
& & 0
\end{array}\right)\left(\begin{array}{rrr}
-1 & -t & -t \\
-t & 0 & -t \\
-t & -t & 0
\end{array}\right)\right) \in V .
$$

Then $\varphi(X(t))=u v(u+v-w)-\gamma w^{3}$ where $\gamma=2 t^{3}-t^{2}$. For $A=\left(a_{i j}\right)$ 
$\in \mathfrak{g} \mathfrak{l}(3), A \varphi(X(t))=a_{12} u^{3}+a_{21} v^{3}-3 \gamma a_{33} v^{3}+\left(2 \dot{a}_{11}+2 a_{12}+a_{22}-a_{13}\right) u^{2} v+\left(a_{32}\right.$ $\left.-a_{12}\right) u^{2} w+\left(2 a_{21}+2 a_{22}+a_{11}-a_{23}\right) v^{2} u+\left(a_{31}-a_{21}\right) v^{2} w-\left(a_{32}+3 \gamma a_{13}\right) w^{2} u-$ $\left(a_{31}+3 a_{23}\right) w^{2} v+\left(2 a_{31}+2 a_{32}-a_{11}-a_{22}-a_{33}\right) u v w$ and hence the isotropy subalgebra at $X_{0}$ is $\{A \in \mathfrak{g} \mathfrak{l}(3) \mid A \varphi(X(t))=0\}=0$ if $\gamma \neq 0$. This implies that if $\gamma \neq 0$, the $G L(3)$-orbit of $\varphi(X(t))$ is of dimension 9. On the other hand, $\varphi(X(t))=0$ is an elliptic curve for $\gamma \neq 0,-\frac{1}{27}$, and its modular invariant is given by $J=\frac{-27 \gamma(1+8 \gamma)^{3}}{\gamma^{3}+27 \gamma^{4}}$. Since $J$ is invariant under the action of $G L(3)$, the dimension of the union of $G L(3)$-orbits of $\varphi(X(t))$ is 10. This implies that $\varphi$ is generically surjective, and hence a triplet $\left(E_{6} \times G L(3), \Lambda_{1} \otimes \Lambda_{1}, V(27) \otimes V(3)\right)$ is not a P.V.

Proposition 50. A triplet $\left(E_{6} \times G L(3), \Lambda_{1} \otimes \Lambda_{1}, V(27) \otimes V(3)\right)$ is not a P.V.

$$
\left(E_{6} \times G L(2), \Lambda_{1} \otimes 2 \Lambda_{1}, V(27) \otimes V(3)\right) .
$$

Since $\left(E_{6} \times G L(2), \Lambda_{1} \otimes 2 \Lambda_{1}\right) \subset\left(E_{6} \times G L(3), \Lambda_{1} \otimes \Lambda_{1}\right)$, it is clearly not a P.V. by Proposition 50.

Proposition 51. A triplet $\left(E_{6} \times G L(3), \Lambda_{1} \otimes 2 \Lambda_{1}, V(27) \otimes V(3)\right)$ is not a P.V.

$$
\left(E_{7} \times G L(d), \Lambda_{6} \otimes \Lambda_{1}, V(56) \otimes V(d)\right) \quad(1 \leq d \leq 2) .
$$

I) The case of $d=1$.

We may identify $V(56) \otimes V(1)$ with $\mathfrak{M}=C \oplus C \oplus \mathscr{J} \oplus \mathscr{J}$ (See Example 40 in $\S 1)$. Let $g_{X_{0}}$ be the isotropy subalgebra of $E_{7} \oplus \operatorname{gr}(1)$ at $X_{0}=$ $(1,1,0,0)$ in $\mathfrak{M}$. For $A=a \oplus \bar{b} \oplus 2 m R_{I} \oplus L \oplus k \in E_{7} \oplus \operatorname{gr}(1)$ where $a, b$ $\in \mathscr{J}, m, k \in C$, and $L \in E_{6}$, by (1.14) we have $\left[a, X_{0}\right]=(0,0, a, 0),\left[\bar{b}, X_{0}\right]$ $=(0,0,0,-b),\left[2 m R_{I}, X_{0}\right]=(3 m,-3 m, 0,0),\left[k, X_{0}\right]=(k, k, 0,0)$ and $\left[L, X_{0}\right]$ $=(0,0,0,0)$, and hence $\left[A, X_{0}\right]=(3 m+k,-3 m+k, a,-b)$. Therefore we have $\mathfrak{g}_{X_{0}}=\left\{A \in E_{7} \oplus \mathfrak{g l}(1) \mid\left[A, X_{0}\right]=0\right\}=\left\{L \in E_{6}\right\} \cong E_{6}$. Since dim $\mathfrak{g}_{X_{0}}$ $=78=\operatorname{dim} E_{7} \times G L(1)(=134)-\operatorname{dim} V(=56)$, it is a regular P.V. by Proposition 25 in $\S 4$. The relative invariant is a quartic form $q(X)$ in (1.16).

The orbital decomposition is completed by S. J. Harris (See [7]).

Proposition 52. A triplet $\left(G L(1) \times E_{7}, \square \otimes \Lambda_{6}, V(1) \otimes V(56)\right)$ is a regular P.V. and its generic isotropy subgroup is locally isomorphic to $E_{6}$. The relative invariant is a quartic form $q(X)$ given in (1.16). 
II) The case of $d=2$.

We may identify $V=V(56) \otimes V(2)$ with $\mathfrak{M} \oplus \mathfrak{M}$. Put $f_{1}(X)=\left\{X_{1}, X_{2}\right\}$ $=\xi_{1} \eta_{2}-\xi_{2} \eta_{1}+T\left(x_{1} y_{2}\right)-T\left(x_{2} y_{1}\right)$ for $X=\left(X_{1}, X_{2}\right)=\left(\left(\xi_{1}, \eta_{1}, x_{1}, y_{1}\right),\left(\xi_{2}, \eta_{2}, x_{2}\right.\right.$, $\left.\left.y_{2}\right)\right) \in V$. Then this is a relative invariant of $E_{7} \times G L(2)$ i.e. $E_{7} \subset S p(28)$ (See Example 40 in $\S 1$ ). Let $q(X)$ be the invariant quartic form of $E_{7}$, i.e., $q(X)=T\left(x^{\sharp}, y^{\sharp}\right)-\xi N(x)-\eta N(y)-\frac{1}{4}(T(x, y)-\xi \eta)^{2}$ for $X=(\xi, \eta, x, y)$ $\in \mathfrak{M}$, where $a^{\sharp}=a^{2}-\operatorname{Tr}(a) \cdot a+\frac{1}{2}\left\{(\operatorname{Tr} a)^{2}-\operatorname{Tr}\left(a^{2}\right)\right\} \cdot 1$ (See Example 40 in $\S 1)$. For each $X=\left(X_{1}, X_{2}\right) \in \mathfrak{M} \oplus \mathfrak{M}$, we have a binary quartic form $\varphi(X)=q\left(u X_{1}+v X_{2}\right)$. In general for a binary quartic form $\tilde{x}=$ $\sum_{i=0}^{4} x_{i} u^{4-i} v^{i}$, one can easily check that the polynomial $h(\tilde{x})=x_{2}^{2}-3 x_{1} x_{3}$ $+12 x_{0} x_{4}$ is relatively invariant under the action of $G L(2)$. Since $\varphi$ is an equivariant map, the polynomial $f_{2}(X)=h(\varphi(X))$ is a relative invariant of $E_{7} \times G L(2)$. Since $\operatorname{deg} f_{1}(X)=2$ and $\operatorname{deg} f_{2}(X)=8$, the quotient $f(X)=$ $f_{1}(X)^{4} \cdot f_{2}(X)^{-1}$ is an absolute invariant. For $X=((1,0,0,0),(0,1,0,0))$ $\in \mathfrak{M} \oplus \mathfrak{M}, f_{1}(X)=1$ and $q((u, v, 0,0))=-\frac{1}{4} u^{2} v^{2}$, i.e., $f_{2}(X)=\frac{1}{16}$, and hence $f(X) \neq 0$. On the other hand, for

$$
X=\left(\left(0,0,\left(\begin{array}{lll}
1 & & \\
& 1 & \\
& & 0
\end{array}\right), 0\right),\left(0,0,0,\left(\begin{array}{lll}
1 & & \\
& -1 & \\
& & 0
\end{array}\right)\right)\right) \in \mathfrak{M} \oplus \mathfrak{M}
$$

we have $f_{1}(X)=0$,

$$
q\left(\left(0,0,\left(\begin{array}{lll}
u & & \\
& u & \\
& & 0
\end{array}\right),\left(\begin{array}{lll}
v & & \\
& -v & \\
& & 0
\end{array}\right)\right)\right)=T\left(\left(\begin{array}{lll}
u & & \\
& u & \\
& & 0
\end{array}\right)^{\#},\left(\begin{array}{lll}
v & & \\
& -v & \\
& & 0
\end{array}\right)\right)=-u^{2} v^{2}
$$

i.e. $f_{2}(X)=1$, and hence $f(X)=0$. This implies that $f(X)$ is a nonconstant absolute invariant, and hence this triplet is not a P.V. by Proposition 3 in $\S 2$.

Proposition 53. A triplet $\left(E_{7} \times G L(2), \Lambda_{6} \otimes \Lambda_{1}, V(56) \otimes V(2)\right)$ is not a P.V.

Theorem 54. Let $(\tilde{G}, \tilde{\rho}, \tilde{V})$ be a reduced P.V. and let $\tilde{g}$ be the Lie algebra of $\tilde{\rho}(\tilde{G})$. Assume that the center of $\tilde{\mathfrak{g}}$ is one-dimensional. Then it is equivalent to one of the following P.V.'s.

I) $A$ regular P.V.

(1) $\left(G \times G L(m), \rho \otimes \Lambda_{1}, V(m) \otimes V(m)\right)$ where $\rho: G \rightarrow G L(V(m))$ is an $m$-dimensional irreducible representation of a semi-simple algebraic 
group $G$.

(2) $\left(G L(n), 2 \Lambda_{1}, V\left(\frac{1}{2} n(n+1)\right) \quad(n \geqq 2)\right.$

(3) $\quad\left(G L(2 m), \Lambda_{2}, V(m(2 m-1))\right) \quad(m \geqq 3)$

(4) $\quad\left(G L(2), 3 \Lambda_{1}, V(4)\right)$

( 5 ) $\left(G L(6), \Lambda_{3}, V(20)\right)$

(6) $\left(G L(7), \Lambda_{3}, V(35)\right)$

( 7$) \quad\left(G L(8), \Lambda_{3}, V(56)\right)$

( 8 ) $\left(S L(3) \times G L(2), 2 \Lambda_{1} \otimes \Lambda_{1}, V(6) \otimes V(2)\right)$

(9) $\left(S L(6) \times G L(2), \Lambda_{2} \otimes \Lambda_{1}, V(15) \otimes V(2)\right)$

(10) $\quad\left(S L(5) \times G L(3), \Lambda_{2} \otimes \Lambda_{1}, V(10) \otimes V(3)\right)$

(11) $\left(S L(5) \times G L(4), \Lambda_{2} \otimes \Lambda_{1}, V(10) \otimes V(4)\right)$

(12) $\quad\left(S L(3) \times S L(3) \times G L(2), \Lambda_{1} \otimes \Lambda_{1} \otimes \Lambda_{1}, V(3) \otimes V(3) \otimes V(2)\right)$

(13) $\left(S p(n) \times G L(2 m), \Lambda_{1} \otimes \Lambda_{1}, V(2 n) \otimes V(2 m)\right) \quad(n \geqq 2 m \geqq 2)$

(14) $\quad\left(G L(1) \times S p(3), \square \otimes \Lambda_{3}, V(1) \otimes V(14)\right)$

(15) $\left(S O(n) \times G L(m), \Lambda_{1} \otimes \Lambda_{1}, V(n) \otimes V(m)\right) \quad(n \geqq 3, n / 2 \geqq m \geqq 1)$

(16) $\quad(G L(1) \times$ Spin (7), $\square \otimes \operatorname{spin~rep.,~} V(1) \otimes V(8))$

(17) $\quad\left(\right.$ Spin $(7) \times G L(2)$, spin rep. $\left.\otimes \Lambda_{1}, V(8) \otimes V(2)\right)$

(18) $\quad\left(\right.$ Spin $(7) \times G L(3)$, spin rep. $\left.\otimes \Lambda_{1}, V(8) \otimes V(3)\right)$

(19) $(G L(1) \times \operatorname{Spin}(9), \square \otimes \operatorname{spin}$ rep., $V(1) \otimes V(16))$

(20) $\quad\left(\right.$ Spin $(10) \times G L(2)$, half-spin rep. $\left.\otimes \Lambda_{1}, V(16) \otimes V(2)\right)$

(21) $\quad\left(\right.$ Spin $(10) \times G L(3)$, half-spin rep. $\left.\otimes \Lambda_{1}, V(16) \otimes V(3)\right)$

(22) $\quad(G L(1) \times \operatorname{Spin}(11), \square \otimes \operatorname{spin}$ rep., $V(1) \otimes V(32))$

(23) $\quad(G L(1) \times \operatorname{Spin}(12), \square \otimes$ half-spin rep., $V(1) \otimes V(32))$

(24) $\quad(G L(1) \times \operatorname{Spin}(14), \square \otimes$ half-spin rep., $V(1) \otimes V(64))$

(25) $\quad\left(G L(1) \times\left(G_{2}\right), \square \otimes \Lambda_{2}, V(1) \otimes V(7)\right)$

(26) $\quad\left(\left(G_{2}\right) \times G L(2), \Lambda_{2} \otimes \Lambda_{1}, V(7) \otimes V(2)\right)$

(27) $\left(G L(1) \times E_{6}, \square \otimes \Lambda_{1}, V(1) \otimes V(27)\right)$

(28) $\quad\left(E_{6} \times G L(2), \Lambda_{1} \otimes \Lambda_{1}, V(27) \otimes V(2)\right)$

(29) $\left(G L(1) \times E_{7}, \square \otimes \Lambda_{6}, V(1) \otimes V(56)\right)$

II) A non-regular P.V. with relative invariants.

$\left(S p(n) \times G L(2), \Lambda_{1} \otimes 2 \Lambda_{1}, V(2 n) \otimes V(3)\right)$

$\cong\left(G L(1) \times S p(n) \times S O(3), \square \otimes \Lambda_{1} \otimes \Lambda_{1}, V(1) \otimes V(2 n) \otimes V(3)\right)$

III) A non-regular P.V. without relative invariants.

(1) $\left(G \times G L(m), \rho \otimes \Lambda_{1}, V(n) \otimes V(m)\right)$ where $\rho: G \rightarrow G L(V(n))$ is an $n$-dimensional irreducible representation of a semi-simple algebraic group $G(\neq S L(n))$ with $m>n \geqq 3$. 
(2) $\left(S L(n) \times G L(m), \Lambda_{1} \otimes \Lambda_{1}, V(n) \otimes V(m)\right) \quad(m / 2 \geqq n \geqq 1)$

(3) $\left(G L(2 m+1), \Lambda_{2}, V(m(2 m+1))\right) \quad(m \geqq 2)$

(4) $\quad\left(S L(2 m+1) \times G L(2), \Lambda_{2} \otimes \Lambda_{1}, V(m(2 m+1)) \otimes V(2)\right) \quad(m \geqq 2)$

(5) $\left(S p(n) \times G L(2 m+1), \Lambda_{1} \otimes \Lambda_{1}, V(2 n) \otimes V(2 m+1)\right)$

$$
(n>2 m+1 \geqq 1)
$$

(6) $\quad(G L(1) \times \operatorname{Spin}(10), \square \otimes h a l f-s p i n$ rep., $\square \otimes V(16))$

Proof. By Theorem 24 in $\S 3$ and Proposition $1 \sim$ Proposition 53, we obtain our assertion.

Q.E.D.

\section{§6. Semi-simple case}

Let $(G, \rho, V)$ be an irreducible P.V. Then the Lie algebra $g$ of $\rho(G)$ is reductive with center at most one-dimensional by Theorem 1 in $\S 1$. In the previous sections, we have considered the case when the center of $\mathrm{g}$ is of one dimension. In this section, we shall consider the case when the center of $\mathfrak{g}$ is of zero dimension. Then $\mathfrak{g}$ is semi-simple and hence $\mathfrak{g} \subset \mathfrak{g l}(V)$. Therefore, there is no relative invariant by Proposition 20 in $\S 4$. Then a triplet $(G L(1) \times G, \square \otimes \rho, V(1) \otimes V)$ is a P.V. without relative invariant and hence by Theorem 54 in $\S 5$, it belongs to the same castling class as one of the following reduced P.V.'s.

(1) $\left(G \times G L(m), \rho \otimes \Lambda_{1}, V(n) \otimes V(m)\right)$ where $\rho$ is any $n$-dimensional irreducible representation of a connected semi-simple algebraic group $G$ $(\neq S L(n))$ with $m>n \geq 3$.

(2) $\left(S L(n) \times G L(m), \Lambda_{1} \otimes \Lambda_{1}, V(n) \otimes V(m)\right)$ with $m / 2 \geq n \geq 1$.

(3) $\left(G L(2 m+1), \Lambda_{2}, V(m(2 m+1))\right)$.

(4) $\left(S L(2 m+1) \times G L(2), \Lambda_{2} \otimes \Lambda_{1}, V(m(2 m+1)) \otimes V(2)\right)$.

(5) $\left.S p(n) \times G L(2 m+1), \Lambda_{1} \otimes \Lambda_{1}, V(2 n) \otimes V(2 m+1)\right)$ with $n \geq 2 m$ $+1 \geq 1$.

(6) $(G L(1) \times$ Spin (10), $\square \otimes$ half-spin rep., $V(1) \otimes V(16))$.

As we have seen in $\S 5$, the generic isotropy subalgebras $\mathfrak{h}$ of these P.V.'s are not contained in $\mathfrak{g l}(V)$, and hence these are P.V. even if without the action of scalar multiplications. Therefore, we obtain the following proposition.

Proposition 1. Let $(\tilde{G}, \tilde{\rho}, \tilde{V})$ be a reduced P.V. where $\tilde{G}$ is a connected semi-simple algebraic group. Then it is equivalent to one of the following reduced $P . V$.'s.

(1) $\left(G \times S L(m), \rho \otimes \Lambda_{1}, V(n) \otimes V(m)\right)$ where $\rho$ is any n-dimensional 
irreducible representation of a connected semi-simple algebraic group $G$ $(\neq S L(n))$ with $m>n \geqq 3$.

(2) $\quad\left(S L(n) \times S L(m), \Lambda_{1} \otimes \Lambda_{1}, V(n) \otimes V(m)\right)$ with $m / 2 \geq n \geq 1$.

(3) $\left(S L(2 m+1), \Lambda_{2}, V(m(2 m+1))\right)$.

(4) $\quad\left(S L(2 m+1) \times S L(2), \Lambda_{2} \otimes \Lambda_{1}, V(m(2 m+1)) \otimes V(2)\right)$.

(5) $\quad\left(S p(n) \times S L(2 m+1), \Lambda_{1} \otimes \Lambda_{1}, V(2 n) \otimes V(2 m+1)\right)$ with $n \geq 2 m$ $+1 \geq 1$.

(6) (Spin (10), half-spin rep., $V(16))$.

\section{§7. Table of irreducible reduced prehomogeneous vector spaces}

In general, we denote by $H$ a generic isotropy subgroup of a reduced P.V. If two groups $H_{1}$ and $H_{2}$ are isomorphic (resp. locally isomorphic) to each other, we denote this relation by $H_{1} \simeq H_{2}$ (resp. $H_{1} \sim H_{2}$ ). The degree of the irreducible relative invariant polynomial $f(x)$ will be denoted by $\operatorname{deg} f$. Then from $\S 1 \sim \S 6$, we obtain the following table.

I) A Regular Prehomogeneous Vector Space.

(1) $\left(G \times G L(m), \rho \otimes A_{1}, V(m) \otimes V(m)\right)$ where $\rho: G \rightarrow G L(V(m))$ is an $m$-dimensional irreducible representation of a connected semisimple algebraic group $G$ (or $G=\{1\}$ and $m=1$ ).

(i) $H \simeq G$, (ii) $\operatorname{deg} f=m$, (iii) $f(x)=\operatorname{det} x$ for $x \in M(m) \simeq V(m)$

$\otimes V(m)$ (see Proposition 1 in $\S 5$ ).

(2) $\quad\left(G L(n), 2 \Lambda_{1}, V\left(\frac{1}{2} n(n+1)\right)\right) \quad(n \geq 2)$.

(i) $H \simeq O(n), \quad$ (ii) $\operatorname{deg} f=n$, (iii) $f(x)=\operatorname{det} x$ for $x \in\left\{\left.x \in M(n)\right|^{t} x\right.$ $=x\} \simeq V\left(\frac{1}{2} n(n+1)\right)$ (see Proposition 3 in $\S 5$ ).

(3) $\quad\left(G L(2 m), \Lambda_{2}, V(m(2 m-1)) \quad(m \geq 3)\right.$.

(i) $H \simeq S p(m), \quad$ (ii) $\operatorname{deg} f=m$, (iii) $f(x)=\operatorname{Pff}(x)$ for $x \in\{x \in$ $\left.M(2 m) \mid{ }^{t} x=-x\right\} \simeq V(m(2 m-1)$ ) (see Proposition 4 in $\S 5$ ).

(4) $\quad\left(G L(2), 3 \Lambda_{1}, V(4)\right)$.

(i) $H \sim\{1\}, \# H=18$, (ii) $\operatorname{deg} f=4$, (iii) $f(x)=x_{2}^{2} x_{3}^{2}+18 x_{1} x_{2} x_{3} x_{4}$ $-4 x_{1} x_{3}^{3}-4 x_{2}^{3} x_{4}-27 x_{1}^{2} x_{4}^{2}$ for $x=x_{1} u^{3}+x_{2} u^{2} v+x_{3} u v^{2}+x_{4} v^{3}$, i.e., the discriminant of a binary cubic form $x$ (see Proposition 6 in $\S 5$ ).

(5) $\quad\left(G L(6), \Lambda_{3}, V(20)\right)$.

(i) $H \simeq(S L(3) \times S L(3)) \cdot\{ \pm 1\}, \quad$ (ii) $\operatorname{deg} f=4, \quad$ (iii) $f(x)=\left(x_{0} y_{0}\right.$ $\operatorname{tr} X Y)^{2}+4 x_{0} \operatorname{det} Y+4 y_{0} \operatorname{det} X-4 \sum_{i, j} \operatorname{det}\left(X_{i j}\right) \cdot \operatorname{det}\left(Y_{j i}\right)$ (see after Proposition 7 in $\S 5$ ).

(6) $\left(G L(7), \Lambda_{3}, V(35)\right)$. 
(i) $H \simeq\left(G_{2}\right) \times\left\{\omega I_{7} \mid \omega^{3}=1\right\}$, (ii) $\operatorname{deg} f=7$ (see Proposition 8 in $\S 5$ ).

(7) $\left(G L(8), \Lambda_{3}, V(56)\right)$.

(i) $H \sim S L(3)$, (ii) $\operatorname{deg} f=16$ (see Proposition 10 in $\S 5$ ).

(8) $\quad\left(S L(3) \times G L(2), 2 \Lambda_{1} \otimes \Lambda_{1}, V(6) \otimes V(2)\right)$.

(i) $H \sim\{1\}, \# H=144$, (ii) $\operatorname{deg} f=12$, (iii) $f(x)$ is given by the discriminant of a binary cubic form $\operatorname{det}(u X+v Y)$ for $x=(X, Y)$ $\in\left\{(X, Y) \mid X, Y \in M(3),{ }^{t} X=X,{ }^{t} Y=Y\right\} \simeq V(6) \otimes V(2)$ (see Proposition 11 in $\S 5)$.

(9) $\quad\left(S L(6) \times G L(2), \Lambda_{2} \otimes \Lambda_{1}, V(15) \otimes V(2)\right)$.

(i) $H \sim S L(2) \times S L(2) \times S L(2)$, (ii) $\operatorname{deg} f=12$, (iii) $f(x)$ is given by the discriminant of a binary cubic form $\operatorname{Pff}(u X+v Y)$ for $x=(X, Y) \in\left\{(X, Y) \mid X, Y \in M(6), \quad{ }^{t} X=-X, \quad{ }^{t} Y=-Y\right\} \simeq V(15) \otimes$ $V(2)$ (see Proposition 12 in $\S 5$ ).

(10) $\quad\left(S L(5) \times G L(3), \Lambda_{2} \otimes \Lambda_{1}, V(10) \otimes V(3)\right)$.

(i) $H \sim S L$ (2), (ii) $\operatorname{deg} f=15$ (see Proposition 14 in $\S 5$ ).

(11) $\quad\left(S L(5) \times G L(4), \Lambda_{2} \otimes \Lambda_{1}, V(10) \otimes V(4)\right)$.

(i) $H \sim\{1\}$, (ii) $\operatorname{deg} f=40$, (iii) $f(x)$ is given by the proof of Proposition 16 in $\S 4$ (see Proposition 15 in $\S 5$ ).

(12) $\quad\left(S L(3) \times S L(3) \times G L(2), \Lambda_{1} \otimes \Lambda_{1} \otimes \Lambda_{1}, V(3) \otimes V(3) \otimes V(2)\right)$.

(i) $H \sim G L(1) \times G L(1)$, (ii) $\operatorname{deg} f=12$, (iii) $f(x)$ is given by the discriminant of a binary cubic form $\operatorname{det}(u X+v Y)$ for $x=(X, Y)$ $\in M(3) \oplus M(3) \simeq V(3) \otimes V(3) \otimes V(2)$.

(13) $\quad\left(S p(n) \times G L(2 m), \Lambda_{1} \otimes \Lambda_{1}, V(2 n) \otimes V(2 m) \quad(n \geq 2 m \geq 2)\right.$.

(i) $H \sim S p(m) \times S p(n-m)$, (ii) $\operatorname{deg} f=2 m$, (iii) $f(x)=P f f\left({ }^{t} X J X\right)$ for $X \in M(2 n, 2 m)$ (see Proposition 17 in $\S 5$ ).

(14) $\quad\left(G L(1) \times S p(3), \square \otimes \Lambda_{3}, V(1) \otimes V(14)\right)$.

(i) $H \sim S L$ (3), (ii) $\operatorname{deg} f=4$, (iii) $f(x)$ is given by the restriction of the relative invariant of $\left(G L(6), \Lambda_{3}, V(20)\right.$ ) (see Proposition 22 in $\S 5)$.

(15) $\quad\left(S O(n) \times G L(m), \Lambda_{1} \otimes \Lambda_{1}, V(n) \otimes V(m)\right) \quad(n \geq 3, n / 2 \geq m \geq 1)$. (i) $H \simeq(S O(m) \times S O(n-m)) \cdot\{ \pm 1\}, \quad$ (ii) $\operatorname{deg} f=2 m$, (iii) $f(X)=$ $\operatorname{det}^{t} X K X$ for $X \in M(n, m) \simeq V(n) \otimes V(m)$ (see Proposition 23 in $\S 5$ ).

(16) $\quad(G L(1) \times \operatorname{Spin}(7), \square \otimes$ spin rep., $V(1) \otimes V(8))$.

(i) $H \sim\left(G_{2}\right)$, (ii) $\operatorname{deg} f=2$, (iii) By the spin representation, we have Spin (7) $\longrightarrow S O(8)$ and hence a relative invariant is that of $\left(G L(1) \times S O(8), \square \otimes \Lambda_{1}, V(1) \otimes V(8)\right)$ (see (15), or Proposition 25 in $\S 5)$. 
(17) $\quad\left(\right.$ Spin (7) $\times G L(2)$, spin rep. $\left.\otimes \Lambda_{1}, V(8) \otimes V(2)\right)$.

(i) $H \sim S L(3) \times O(2)$, (ii) $\operatorname{deg} f=4$, (iii) By the spin representation,- we have Spin (7) $\longrightarrow S O(8)$ and hence a relative invariant is that of $\left(S O(8) \times G L(2), \Lambda_{1} \otimes \Lambda_{1}, V(8) \otimes V(2)\right)$ (see (15), or Proposition 26 in $\S 5)$.

(18) $\quad\left(\right.$ Spin $(7) \times G L(3)$, spin rep. $\left.\otimes \Lambda_{1}, V(8) \otimes V(3)\right)$.

(i) $H \sim S L(2) \times O(3)$, (ii) $\operatorname{deg} f=6$, (iii) By the spin representation, we have Spin $(7) \smile S O(8)$ and hence a relative invariant is that of $\left(S O(8) \times G L(3), \Lambda_{1} \otimes \Lambda_{1}, V(8) \otimes V(3)\right)$ (see (15), or Proposition 27 in $\S 5)$.

(19) $\quad(G L(1) \times \operatorname{Spin}(9), \quad \square \otimes \operatorname{spin}$ rep., $V(1) \otimes V(16))$.

(i) $H \sim \operatorname{Spin}(7)$, (ii) $\operatorname{deg} f=2$, (iii) $f(x)=x_{0} y_{0}+P f f\left(x_{i j}\right)+\sum_{i} x_{i} y_{i}$ for $x=x_{0}+\sum_{i<j \leq 4} x_{i j} e_{i} e_{j}+y_{0} e_{1} e_{2} e_{3} e_{4}+\left(\sum_{i} x_{i} e_{i}+\sum_{i} y_{i} e_{i}^{*}\right) e_{5}$ where $e_{i}^{*} e_{i}=e_{1} e_{2} e_{3} e_{4} e_{5}$ for $1 \leq i \leq 4$ (see Proposition 35 in $\S 5$ ).

(20) $\quad\left(\right.$ Spin $(10) \times G L(2)$, half-spin rep. $\left.\otimes \Lambda_{1}, V(16) \otimes V(2)\right)$.

(i) $H \sim\left(G_{2}\right) \times S L(2)$, (ii) $\operatorname{deg} f=4$ (see Proposition 32).

(21) $\quad\left(\right.$ Spin $(10) \times G L(3)$, half-spin rep. $\left.\otimes \Lambda_{1}, V(16) \otimes V(3)\right)$.

(i) $H \sim S L(2) \times O(3)$, (ii) $\operatorname{deg} f=12$ (see Proposition 33 in $\S 5$ ).

(22) $\quad(G L(1) \times \operatorname{Spin}(11), \square \otimes \operatorname{spin}$ rep., $V(1) \otimes V(32))$.

(i) $H \sim S L(5)$, (ii) $\operatorname{deg} f=4$, (iii) $f(x)=x_{0} \operatorname{Pff}\left(\left(y_{i j}\right)\right)+y_{0} \operatorname{Pff}\left(\left(x_{i j}\right)\right)$ $+\sum_{i<j} P f f\left(X_{i j}\right) \cdot \operatorname{Pff}\left(Y_{i j}\right)-\frac{1}{4}\left(x_{0} y_{0}-\sum_{i<j} x_{i j} y_{i j}\right)^{2}$ for $x=x_{0}+$ $\sum_{i<j} x_{i j} e_{i} e_{j}+\sum_{i<j} y_{i j} e_{i j}^{*}+y_{0} e_{1} e_{2} e_{3} e_{4} e_{5} e_{6}$ where $e_{i j}^{*}=(-1)^{i+j-1} e_{1} \cdots$ $e_{i-1} e_{i+1} \cdots e_{j-1} e_{j+1} \cdots e_{6}$ (see Proposition 39 in $\S 5$ ).

(23) $\quad(G L(1) \times \operatorname{Spin}(12), \square \otimes h a l f-s p i n$ rep., $V(1) \otimes V(32))$.

(i) $H \sim S L(6)$, (ii) $\operatorname{deg} f=4$, (iii) $f(x)$ is the same as (22) (see Proposition 37 in $\S 5$ ).

(24) $(G L(1) \times \operatorname{Spin}(14), \square \otimes h a l f-s p i n$ rep., $V(1) \otimes V(64))$.

(i) $H \sim\left(G_{2}\right) \times\left(G_{2}\right)$, (ii) $\operatorname{deg} f=8$ (see Proposition 40 in $\S 5$ ).

(25) $\quad\left(\left(G L(1) \times\left(G_{2}\right), \square \otimes A_{2}, V(1) \otimes V(7)\right)\right.$.

(i) $H \sim S L(3)$, (ii) $\operatorname{deg} f=2$, (iii) By $\Lambda_{2}$, we have $\left(G_{2}\right) \smile S O(7)$ and hence a relative invariant is that of $\left(G L(1) \times S O(7), \square \otimes \Lambda_{1}\right.$, $V(1) \otimes V(7))$ (see Proposition 42 in $\S 5$ ).

(26) $\quad\left(\left(G_{2}\right) \times G L(2), \Lambda_{2} \otimes \Lambda_{1}, V(7) \otimes V(2)\right)$.

(i) $H \sim G L(2), \quad$ (ii) $\operatorname{deg} f=4$, (iii) $\mathrm{By} \Lambda_{2}$, we have $\left(G_{2}\right) \smile S O(7)$ and hence a relative invariant is that of $\left(S O(7) \times G L(2), \Lambda_{1} \otimes \Lambda_{1}\right.$, $V(7) \otimes V(2))$ (see (15), or Proposition 43 in $\S 5)$. 
(27) $\left(G L(1) \times E_{6}, \square \otimes \Lambda_{1}, V(1) \otimes V(27)\right)$.

(i) $H \sim F_{4}$, (ii) $\operatorname{deg} f=3$, (iii) $f(x)=\operatorname{det} x=\xi_{1} \xi_{2} \xi_{3}+\operatorname{tr} x_{1} x_{2} x_{3}$ $\xi_{1} x_{1} \bar{x}_{1}-\xi_{2} x_{2} \bar{x}_{2}-\xi_{3} x_{3} \bar{x}_{3}$ for

$$
x=\left(\begin{array}{lll}
\xi_{1} & x_{3} & \bar{x}_{2} \\
\bar{x}_{3} & \xi_{2} & x_{1} \\
x_{2} & \bar{x}_{1} & \xi_{3}
\end{array}\right) \in \mathscr{J} \simeq V(1) \otimes V(27)
$$

(see Proposition 47 in $\S 5$ ).

(28) $\quad\left(E_{6} \times G L(2), \Lambda_{1} \otimes \Lambda_{1}, V(27) \otimes V(2)\right)$.

(i) $H \sim O(8)$, (ii) $\operatorname{deg} f=12$, (iii) $f(x)$ is given by the discriminant of a binary cubic form $\operatorname{det}(u X+v Y)$ for $x=(X, Y) \in \mathscr{J} \oplus \mathscr{J}$ $\simeq V(27) \otimes V(2)$ (see Proposition 48 in $\S 5$ ).

(29) $\quad\left(G L(1) \times E_{7}, \square \otimes \Lambda_{6}, V(1) \otimes V(56)\right)$.

(i) $H \sim E_{6}, \quad$ (ii) $\operatorname{deg} f=4$, (iii) $f(X)=T\left(x^{\sharp}, y^{\sharp}\right)-\xi N(x)-\eta N(y)$ $-\frac{1}{4}(T(x, y)-\xi \eta)^{2}$ (see (1.16), or Proposition 52 in $\left.\S 5\right)$.

II) A Non-regular Prehomogeneous Vector Space, with relative invariants.

$\left(G L(1) \times S p(n) \times S O(3), \square \otimes \Lambda_{1} \otimes \Lambda_{1}, V(1) \otimes V(2 n) \otimes V(3)\right)$.

(i) $H \sim(S p(n-2) \times S O(2)) \cdot U(2 n-3)$, (ii) $\operatorname{deg} f=4$, (iii) $f(X)$ $=\operatorname{tr}\left({ }^{t} X J X K\right)^{2}$ for $X \in M(2 n, 3) \simeq V(1) \otimes V(2 n) \otimes V(3)$ (see Proposition 19 in $\S 5)$.

III) A Non-regular Prehomogeneous Vector Space, without relative invariant.

(1) $\left(G \times G L(m), \rho \otimes \Lambda_{1}, V(n) \otimes V(m)\right)$.

( $\left.1^{\prime}\right) \quad\left(G \times S L(m), \rho \otimes \Lambda_{1}, V(n) \otimes V(m)\right)$, where $\rho: G \rightarrow G L(V(n))$ is an $n$-dimensional irreducible representation of a semi-simple algebraic group $G(\neq S L(n))$ with $m>n$ $\geq 3$.

(2) $\quad\left(S L(n) \times G L(m), \Lambda_{1} \otimes \Lambda_{1}, V(n) \otimes V(m)\right) \quad(m / 2 \geq n \geq 1)$.

(2') $\quad\left(S L(n) \times S L(m), \Lambda_{1} \otimes \Lambda_{1}, V(n) \otimes V(m)\right)$.

(3) $\quad\left(G L(2 m+1), \Lambda_{2}, V(m(2 m+1)) \quad(m \geq 2)\right.$.

(3') $\quad\left(S L(2 m+1), \Lambda_{2}, V(m(2 m+1))\right)$.

(4) $\quad\left(S L(2 m+1) \times G L(2), \Lambda_{2} \otimes \Lambda_{1}, V(m(2 m+1)) \otimes V(2)\right) \quad(m \geq 2)$.

(4') $\quad\left(S L(2 m+1) \times S L(2), \Lambda_{2} \otimes \Lambda_{1}, V(m(2 m+1)) \otimes V(2)\right)$.

(5) $\quad\left(S p(n) \times G L(2 m+1), \Lambda_{1} \otimes \Lambda_{1}, V(2 n) \otimes V(2 m+1)\right)$

$(n>2 m+1 \geq 1)$. 
( 5') $\quad\left(S p(n) \times S L(2 m+1), \Lambda_{1} \otimes \Lambda_{1}, V(2 n) \otimes V(2 m+1)\right)$.

(6) $(G L(1) \times \operatorname{Spin}(10), \square \otimes$ half-spin rep., $V(1) \otimes V(16))$.

(6') (Spin.(10), half-spin rep., $V(16))$.

\section{§8. Prehomogeneous vector spaces with finitely many orbits}

In this section, we shall study some necessary conditions that a number of orbits of a given irreducible triplet $(G, \rho, V)$ is finite. Clearly, such a triplet must be a P.V. from the dimension reasons. By Theorem 1 in $\S 1$, the Lie algebra $g$ of $\rho(G)$ is reductive with at most one-dimensional center. First we shall consider the case when the center of $g$ is of one dimension. Then as we have seen in $\S 3$, we may assume that $G=G L(1) \times G_{1} \times \cdots \times G_{k}, \rho=\square \otimes \rho_{1} \otimes \cdots \otimes \rho_{k}, V=V(1) \otimes V\left(d_{1}\right) \otimes \cdots$ $\otimes V\left(d_{k}\right)$ with $d_{1} \geq d_{2} \geq \cdots \geq d_{k} \geq 2$, where each $G_{i}$ is a connected almost simple algebraic group, $\rho_{i}$ is an irreducible representation of $G_{i}$ on the $d_{i}$-dimensional vector space $V\left(d_{i}\right)(1 \leq i \leq k)$, and $\square$ is the standard representation of $G L(1)$ on the one-dimensional vector space $V(1)$.

Proposition 1. Let $\rho: G^{\prime} \rightarrow G L\left(V^{\prime}\right)$ be an irreducible representation of a semi-simple algebraic group $G^{\prime}$ on $V^{\prime}$. Assume that a number of orbits of a triplet $\left(G^{\prime} \times G L(n), \rho \otimes \Lambda_{1}, V^{\prime} \otimes V(n)\right)$ is finite. Then for any $k \leq n$, a number of orbits of a triplet $\left(G^{\prime} \times G L(k), \rho \otimes \Lambda_{1}, V^{\prime} \otimes V(k)\right)$ is also finite.

Proof. Identify $V=V^{\prime} \otimes V(n)$ with $V^{\prime} \oplus \cdots \oplus V^{\prime}$ (n-copies) and for any point $v=\left(v_{1}, \cdots, v_{n}\right)$ of $V$, let $\varphi(v)$ be a subspace of $V^{\prime}$ generated by $v_{1}, \cdots, v_{n}$. Then $\varphi$ is a surjective map from $V$ to a set $T=$ $\operatorname{Grass}_{\ell}\left(V^{\prime}\right) \cup \cdots \cup \operatorname{Grass}_{0}\left(V^{\prime}\right)$ where $\ell=\min \left(\operatorname{dim} V^{\prime}, n\right)$ and $\operatorname{Grass}_{r}\left(V^{\prime}\right)$ denotes the Grassmann variety consisting of $r$-dimensional subspaces of $V^{\prime}$. Since $G L(n)$ acts homogeneously on each fibre of $\varphi$, there is a oneto-one correspondence between the orbits of a triplet $\left(G^{\prime} \times G L(n), \rho \otimes\right.$ $\Lambda_{1}, V^{\prime} \otimes V(n)$ ) and the orbits of $G^{\prime}$ on $T$. Therefore, by assumption, a number of $G$-orbits on $\operatorname{Grass}_{r}\left(V^{\prime}\right)(0 \leq r \leq \ell)$ is finite. In particular, for $\ell^{\prime}=\min \left(\operatorname{dim} V^{\prime}, k\right)$, a number of $G$-orbits on $T^{\prime}=\operatorname{Grass}_{\ell^{\prime}}\left(V^{\prime}\right) \cup \ldots$ $\cup \operatorname{Grass}_{0}\left(V^{\prime}\right)$ is finite, and hence we obtain our assertion.

Q.E.D.

Proposition 2. Let $(G, \rho, V)$ be an irreducible P.V. with finitely many orbits. Then we have $0 \leq k \leq 3$.

Proof. Assume that $k \geq 4$. Then a triplet $\left(S L\left(d_{1}\right) \times S L\left(d_{2}\right) \times S L\left(d_{3}\right)\right.$ 
$\times G L\left(d_{4} \cdots d_{k}\right), \Lambda_{1} \otimes \Lambda_{1} \otimes \Lambda_{1} \otimes \Lambda_{1}, V\left(d_{1}\right) \otimes V\left(d_{2}\right) \otimes V\left(d_{3}\right) \otimes V\left(d_{4} \cdots d_{k}\right)$ has finitely many orbits. Therefore by Proposition 1, a triplet $\left(S L\left(d_{1}\right) \times\right.$ $\left.S L\left(d_{2}\right) \times S L\left(d_{3}\right) \times G L(2), \Lambda_{1} \otimes \Lambda_{1} \otimes \Lambda_{1} \otimes \Lambda_{1}, V\left(d_{1}\right) \otimes V\left(d_{2}\right) \otimes V\left(d_{3}\right) \otimes V(2)\right)$ has also finitely many orbits. By repeating this procedure, a triplet $(S L(2)$ $\left.\times S L(2) \times S L(2) \times G L(2), \Lambda_{1} \otimes \Lambda_{1} \otimes \Lambda_{1} \otimes \Lambda_{1}, V(2) \otimes V(2) \otimes V(2) \otimes V(2)\right)$ has finitely many orbits. This is a contradiction since $\operatorname{dim} S L(2) \times S L(2) \times$ $S L(2) \times G L(2)=13<\operatorname{dim} V(2) \otimes V(2) \otimes V(2) \otimes V(2)=16 . \quad$ Q.E.D.

Assume that a number of orbits of a triplet $(G, \rho, V)$ is finite. If $k \leq 1$, then it is a reduced P.V. and hence by Theorem 54 in $\S 5$, we obtain the following assertion.

Proposition 3. If $k \leq 1$, it is equivalent to one of the following P.V.'s.

I) A Regular P.V.

(1) $(G L(1), \square, V(1)) \quad(k=0)$.

(2) $\quad\left(G L(n), 2 \Lambda_{1}, V\left(\frac{1}{2} n(n+1)\right)\right) \quad(n \geq 2)$.

(3) $\quad\left(G L(2 m), A_{2}, V(m(2 m-1))\right) \quad(m \geq 3)$.

(4) $\left(G L(2), 3 \Lambda_{1}, V(4)\right)$.

( 5 ) $\left(G L(6), \Lambda_{3}, V(20)\right)$.

(6) $\left(G L(7), \Lambda_{3}, V(35)\right)$.

( 7) $\left(G L(8), \Lambda_{3}, V(56)\right)$.

(8) $\left(G L(1) \times S p(3), \square \otimes \Lambda_{3}, V(1) \otimes V(14)\right)$.

(9) $\quad\left(G L(1) \times S O(n), \square \otimes \Lambda_{1}, V(1) \otimes V(n)\right) \quad(n \geq 3)$.

(10) $\quad(G L(1) \times \operatorname{Spin}(7), \square \otimes \operatorname{spin}$ rep., $V(1) \otimes V(8))$.

(11) $\quad(G L(1) \times \operatorname{Spin}(9), \square \otimes \operatorname{spin}$ rep., $V(1) \otimes V(16))$.

(12) $(G L(1) \times \operatorname{Spin}(11), \square \otimes \operatorname{spin}$ rep., $V(1) \otimes V(32))$.

(13) $(G L(1) \times \operatorname{Spin}(12), \square \otimes$ half-spin rep., $V(1) \otimes V(32))$.

(14) $(G L(1) \times \operatorname{Spin}(14), \square \otimes$ half-spin rep., $V(1) \otimes V(64))$.

(15) $\quad\left(G L(1) \times\left(G_{2}\right), \square \otimes \Lambda_{2}, V(1) \otimes V(7)\right)$.

(16) $\quad\left(G L(1) \times E_{6}, \square \otimes \Lambda_{1}, V(1) \otimes V(27)\right)$.

(17) $\left(G L(1) \times E_{7}, \square \otimes \Lambda_{6}, V(1) \otimes V(56)\right)$.

II) A Non-regular P.V. without Relative Invariants.

(1) $\left(G L(n), \Lambda_{1}, V(n)\right) \quad(n \geq 2)$.

(2) $\quad\left(G L(2 m+1), \Lambda_{2}, V(m(2 m+1))\right) \quad(m \geq 2)$.

(3) $\quad\left(G L(1) \times S p(n), \square \otimes \Lambda_{1}, V(1) \otimes V(2 n)\right) \quad(n \geq 2)$.

(4) $(G L(1) \times \operatorname{Spin}(10), \square \otimes h a l f-s p i n$ rep., $V(1) \otimes V(16))$. 
Now we shall consider the case of $k=2$. Then it is reduced or is a castling transform of a reduced P.V. with $k \leq 2$. First we shall consider a castling transform of a P.V. with $k=1$.

Proposition 4. The following non-reduced P.V.'s have infinitely many orbits.

I) A Regular P.V.

(1) $\quad\left(S L(n) \times G L\left(\frac{1}{2} n(n+1)-1\right), \quad 2 \Lambda_{1} \otimes \Lambda_{1}, \quad V\left(\frac{1}{2} n(n+1)\right) \otimes V\left(\frac{1}{2} n(n+1)\right.\right.$ - 1)) $\quad(n \geq 3)$.

(2) $\quad\left(S L(2 m) \times G L(m(2 m-1)-1), \Lambda_{2} \otimes \Lambda_{1}, V(m(2 m-1)) \otimes V(m(2 m-\right.$ 1) -1$)) \quad(m \geq 3)$.

(3) $\quad\left(S L(2) \times G L(3), 3 \Lambda_{1} \otimes \Lambda_{1}, V(4) \otimes V(3)\right)$.

(4) $\quad\left(S L(6) \times G L(19), \Lambda_{3} \otimes \Lambda_{1}, V(20) \otimes V(19)\right)$.

( 5 ) $\quad\left(S L(7) \times G L(34), \Lambda_{3} \otimes \Lambda_{1}, V(35) \otimes V(34)\right)$.

(6) $\quad\left(S L(8) \times G L(55), \Lambda_{3} \otimes \Lambda_{1}, V(56) \otimes V(55)\right)$.

(7) $\left.S p(3) \times G L(13), \Lambda_{3} \otimes \Lambda_{1}, V(14) \otimes V(13)\right)$.

(8) $\quad\left(\right.$ Spin $(7) \times G L(7)$, spin rep. $\left.\otimes \Lambda_{1}, V(8) \otimes V(7)\right)$.

(9) $\quad\left(\operatorname{Spin}(9) \times G L(15)\right.$, spin rep. $\left.\otimes \Lambda_{1}, V(16) \otimes V(15)\right)$.

(10) $\quad\left(\right.$ Spin $(11) \times G L(31)$, spin rep. $\left.\otimes \Lambda_{1}, V(32) \otimes V(31)\right)$.

(11) $\quad$ (Spin (12) $\times G L(31)$, half-spin rep. $\left.\otimes \Lambda_{1}, V(32) \otimes V(31)\right)$.

(12) $\quad\left(\right.$ Spin $(14) \times G L(63)$, half-spin rep. $\left.\otimes \Lambda_{1}, V(64) \otimes V(63)\right)$.

(13) $\quad\left(\left(G_{2}\right) \times G L(6), \Lambda_{2} \otimes \Lambda_{1}, V(7) \otimes V(6)\right)$.

(14) $\quad\left(E_{6} \times G L(26), \Lambda_{1} \otimes \Lambda_{1}, V(27) \otimes V(26)\right)$.

(15) $\quad\left(E_{7} \times G L(55), \Lambda_{6} \otimes \Lambda_{1}, V(56) \otimes V(55)\right)$.

II) A Non-regular P.V. without Relative Invariants.

(1) $\left(S L(2 m+1) \times G L(m(2 m+1)-1), \Lambda_{2} \otimes \Lambda_{1}, V(m(2 m+1))\right.$

$\otimes V(m(2 m+1)-1)) \quad(m \geq 2)$.

(2) $\quad\left(\operatorname{Spin}(10) \times G L(15)\right.$, half-spin rep. $\left.\otimes \Lambda_{1}, V(16) \otimes V(15)\right)$.

Proof. Assume that (1) has finitely many orbits. Then by Proposition 1 , a triplet $\left(S L(n) \times G L(3), 2 \Lambda_{1} \otimes \Lambda_{1}, V\left(\frac{1}{2} n(n+1)\right) \otimes V(3)\right)$ has also finitely many orbits. Since $\operatorname{dim} S L(n) \times G L(3)<\operatorname{dim} V\left(\frac{1}{2} n(n+1)\right) \otimes V(3)$, it is not a P.V. and hence it has infinitely many orbits, i.e., a contradiction. Similarly, we can prove that other P.V.'s in Proposition 4 have infinitely many orbits.

Q.E.D.

Next we shall consider a trivial P.V. $\left(G \times G L(m), \rho \otimes \Lambda_{1}, V(n) \otimes\right.$ $V(m))$ with $k=2, m \geq n$. If a number of orbits of this P.V. is finite, 
then by Proposition 1, a triplet $(G L(1) \times G, \square \otimes \rho, V(1) \otimes V(n))$ and a triplet $\left(G \times G L(n-1), \rho \otimes \Lambda_{1}, V(n) \otimes V(n-1)\right)$ have also finitely many orbits. Therefore, by Proposition 3 and Proposition 4 , we have $G=$ $S L(n), S O(n)$, or $S p\left(n^{\prime}\right)\left(n=2 n^{\prime}\right.$ is even). Now we shall consider a castling transform of a reduced P.V. with $k=2$.

Proposition 5. The following non-reduced P.V.'s have infinitely many orbits.

I) $A$ Regular P.V.

(1) $\left(S L(3) \times G L(4), 2 \Lambda_{1} \otimes \Lambda_{1}, V(6) \otimes V(4)\right)$.

(2) $\left(S L(6) \times G L(13), \Lambda_{2} \otimes \Lambda_{1}, V(15) \otimes V(13)\right)$.

(3) $\quad\left(\operatorname{Spin}(7) \times G L(6)\right.$, spin rep. $\left.\otimes \Lambda_{1}, V(8) \otimes V(6)\right)$.

(4) $\left(\right.$ Spin $(7) \times G L(5)$, spin rep. $\left.\otimes \Lambda_{1}, V(8) \otimes V(5)\right)$.

(5) (Spin (10) $\times G L(14)$, half-spin rep. $\left.\otimes \Lambda_{1}, V(16) \otimes V(14)\right)$.

(6) (Spin (10) $\times G L(13)$, half-spin rep. $\left.\otimes \Lambda_{1}, V(16) \otimes V(13)\right)$.

( 7 ) $\quad\left(\left(G_{2}\right) \times G L(5), \Lambda_{2} \otimes \Lambda_{1}, V(7) \otimes V(5)\right)$.

(8) $\left(E_{6} \times G L(25), \Lambda_{1} \otimes \Lambda_{1}, V(27) \otimes V(25)\right)$.

II) A Non-regular P.V.

(9) $\quad\left(S L(2 m+1) \times G L(m(2 m+1)-2), \Lambda_{2} \otimes \Lambda_{1}, V(m(2 m+1))\right.$

$\otimes V(m(2 m+1)-2))$.

Proof. If (3) or (4) has finitely many orbits, then a triplet (Spin (7) $\times G L(4)$, spin rep. $\left.\otimes \Lambda_{1}, V(8) \otimes V(4)\right)$ has also finitely many orbits by Proposition 1. However, this triplet is not a P.V. by Proposition 28 in $\S 5$, i.e., a contradiction and hence (3) and (4) has infinitely many orbits. If (5) or (6) has finitely many orbits, a triplet (Spin (10) $\times G L(4)$, halfspin rep. $\left.\otimes \Lambda_{1}, V(16) \otimes V(4)\right)$ has also finitely many orbits. Since $\operatorname{dim} \operatorname{Spin}(10) \times G L(4)=61<64=\operatorname{dim} V(16) \otimes V(4)$, this triplet is not a P.V., i.e., a contradiction. Similarly, we can prove our assertion for (1), (2), (7), (8).

Q.E.D.

Proposition 6. Let $(G, \rho, V)$ be a triplet with finitely many orbits and $k=2$. Then it is equivalent to one of the following triplets. (Note that we have assumed that the center of $\rho(G)$ is one-dimensional.)

I) A Regular P.V.

(1) $\quad\left(S L(n) \times G L(n), \Lambda_{1} \otimes \Lambda_{1}, V(n) \otimes V(n)\right) \quad(n \geq 2)$.

(2) $\quad\left(S O(n) \times G L(m), \Lambda_{1} \otimes \Lambda_{1}, V(n) \otimes V(m)\right) \quad(n \geq m \geq 2)$. 
(3) $\quad\left(S p(n) \times G L(2 m), \Lambda_{1} \otimes \Lambda_{1}, V(2 n) \otimes V(2 m)\right) \quad(n \geq m \geq 1)$.

(4) $\quad\left(S L(3) \times G L(2), 2 \Lambda_{1} \otimes \Lambda_{1}, V(6) \otimes V(2)\right)$.

(5) $\quad\left(S L(6) \times G L(2), \Lambda_{2} \otimes \Lambda_{1}, V(15) \otimes V(2)\right)$.

(6) $\quad\left(S L(5) \times G L(3), \Lambda_{2} \otimes \Lambda_{1}, V(10) \otimes V(3)\right)$.

( 7 ) $\quad\left(S L(5) \times G L(4), \Lambda_{2} \otimes \Lambda_{1}, V(10) \otimes V(4)\right)$.

(8) $\quad\left(\right.$ Spin $(7) \times G L(2)$, spin rep. $\left.\otimes A_{1}, V(8) \otimes V(2)\right)$.

(9) $\quad\left(\right.$ Spin $(7) \times G L(3)$, spin rep. $\left.\otimes \Lambda_{1}, V(8) \otimes V(3)\right)$.

(10) $\quad\left(\right.$ Spin $(10) \times G L(2)$, half-spin rep. $\left.\otimes \Lambda_{1}, V(16) \otimes V(2)\right)$.

(11) $\quad\left(\right.$ Spin $(10) \times G L(3)$, half-spin rep. $\left.\otimes \Lambda_{1}, V(16) \otimes V(3)\right)$.

(12) $\quad\left(\left(G_{2}\right) \times G L(2), \Lambda_{2} \otimes \Lambda_{1}, V(7) \otimes V(2)\right)$.

(13) $\quad\left(E_{6} \times G L(2), \Lambda_{1} \otimes \Lambda_{1}, V(27) \otimes V(2)\right)$.

II) A Non-regular P.V. with Relative Invariants.

$\left(G L(1) \times S p(n) \times S O(3), \square \otimes \Lambda_{1} \otimes \Lambda_{1}, V(1) \otimes V(2 n) \otimes V(3)\right)$.

III) A Non-regular P.V. without Relative Invariants.

(1) $\quad\left(S L(n) \times G L(m), \Lambda_{1} \otimes \Lambda_{1}, V(n) \otimes V(m)\right) \quad(n<m)$.

(2) $\quad\left(S O(n) \times G L(m), \Lambda_{1} \otimes \Lambda_{1}, V(n) \otimes V(m)\right) \quad(n<m)$,

(3) $\quad\left(S p(n) \times G L(2 m), \Lambda_{1} \otimes \Lambda_{1}, V(2 n) \otimes V(2 m)\right) \quad(n<m)$.

(4) $\quad\left(S p(n) \times G L(2 m+1), \Lambda_{1} \otimes \Lambda_{1}, V(2 n) \otimes V(2 m+1)\right)$.

(5) $\quad\left(S L(2 m+1) \times G L(2), \Lambda_{2} \otimes \Lambda_{1}, V(m(2 m+1)) \otimes V(2)\right) \quad(m \geq 2)$.

Proof. From Theorem 54 in $\S 5$, Propositions 4 and 5, we obtain our assertion. $\quad$ Q.E.D.

Finally, we shall consider the case of $k=3$. Assume that a triplet $\left(G L(1) \times G_{1} \times G_{2} \times G_{3}, \square \otimes \rho_{1} \otimes \rho_{2} \otimes \rho_{3}, V(1) \otimes V\left(d_{1}\right) \otimes V\left(d_{2}\right) \otimes V\left(d_{3}\right)\right)$ has finitely many orbits. Then clearly a triplet $\left(S L\left(d_{1}\right) \times S L\left(d_{2}\right) \times G L\left(d_{3}\right)\right.$, $\left.\Lambda_{1} \otimes \Lambda_{1} \otimes \Lambda_{1}, V\left(d_{1}\right) \otimes V\left(d_{2}\right) \otimes V\left(d_{3}\right)\right)$ has also finitely many orbits and hence by Proposition 1, $\left(S L\left(d_{2}\right) \times S L\left(d_{2}\right) \times G L(2), \Lambda_{1} \otimes \Lambda_{1} \otimes \Lambda_{1}, V\left(d_{2}\right) \otimes V\left(d_{2}\right) \otimes\right.$ $V(2))$ has also finitely many orbits. By Proposition 16 in $\S 5$, it is not a P.V. if $d_{2} \geq 4$, and hence we have $3 \geq d_{2} \geq 2$. If $d_{2}=2$, then our triplet is equivalent to a triplet $\left(G L(1) \times G_{1} \times S O(4), \square \otimes \rho_{1} \otimes \Lambda_{1}, V(1)\right.$ $\left.\otimes V\left(d_{1}\right) \otimes V(4)\right)$. If $G_{1} \neq S L\left(d_{1}\right)$, then this is a reduced P.V., i.e., a contradiction in view of Theorem 54 in $\S 5$. Therefore, $G_{1}=S L\left(d_{1}\right)$. Assume that $d_{2}=3$. If $d_{3}=3$, then a triplet $(S L(3) \times S L(3) \times G L(3)$, $\left.\Lambda_{1} \otimes \Lambda_{1} \otimes \Lambda_{1}, V(3) \otimes V(3) \otimes V(3)\right)$ has also finitely many orbits. This is a contradiction since $\operatorname{dim} S L(3) \times S L(3) \times G L(3)=25<27=\operatorname{dim} V(3) \otimes$ $V(3) \otimes V(3)$, and hence we have $d_{3}=2, G_{3}=S L(2), \rho_{3}=\Lambda_{1}$. Since $d_{2}=3$, 
we have $G_{2}=S L(2), \rho_{2}=2 \Lambda_{1}$ or $G_{2}=S L(3), \rho_{2}=\Lambda_{1}$. Assume that $G_{2}=$ $S L(2)$. Then this triplet is equivalent to $\left(G_{1} \times S O(3) \times G L(2), \rho_{1} \otimes \Lambda_{1} \otimes\right.$ $\left.\Lambda_{1}, V\left(d_{1}\right) \otimes V(3) \otimes V(2)\right)\left(d_{1} \geq 3\right)$. If $G_{1} \neq S L\left(d_{1}\right)$, then it is a reduced P.V., i.e., a contradiction in view of Theorem 54 in $\S 5$ and we have $\left(S L\left(d_{1}\right) \times S O(3) \times G L(2), \Lambda_{1} \otimes \Lambda_{1} \otimes \Lambda_{1}, V\left(d_{1}\right) \otimes V(3) \otimes V(2)\right)$. Since $\left(d_{1}^{2}-1\right)$ $+3+4 \geq 6 d_{1}$, we have $d_{1} \geq 5$. Note that it is regular if $6 \geq d_{1} \geq 5$ and not regular if $d_{1} \geq 7$. Assume that $G_{2}=S L(3)$. Then a triplet $\left(G_{1} \times G L(3), \rho_{1} \otimes \Lambda_{1}, V\left(d_{1}\right) \otimes V(3)\right)$ has finitely many orbits by Proposition 1. Therefore, by Proposition $6, G_{1}=S L\left(d_{1}\right), S O\left(d_{1}\right), S p\left(d_{1}^{\prime}\right) \quad\left(d_{1}=2 d_{1}^{\prime}\right.$ : even), $S L(5)\left(d_{1}=10\right)$, Spin (7) $\left(d_{1}=8\right)$ or $\operatorname{Spin}(10)\left(d_{1}=16\right)$. However, if $G_{1}$ $\neq S L\left(d_{1}\right)$, then $\left(G_{1} \times S L(3) \times G L(2), \rho_{1} \otimes \Lambda_{1} \otimes \Lambda_{1}, V\left(d_{1}\right) \otimes V(3) \otimes V(2)\right)$ is a reduced P.V., i.e., a contradiction in view of Theorem 54 in $\S 4$. Hence we have $\left(S L\left(d_{1}\right) \times S L(3) \times G L(2), \Lambda_{1} \otimes \Lambda_{1} \otimes \Lambda_{1}, V\left(d_{1}\right) \otimes V(3) \otimes V(2)\right)$.

Proposition 7. If $k=3$, then it is equivalent to one of the following triplets.

(1) $\left(S L\left(d_{1}\right) \times S L(2) \times G L(2), \Lambda_{1} \otimes \Lambda_{1} \otimes \Lambda_{1}, V\left(d_{1}\right) \otimes V(2) \otimes V(2)\right) \simeq(S O(4)$ $\left.\times G L\left(d_{1}\right), \Lambda_{1} \otimes \Lambda_{1}, V(4) \otimes V\left(d_{1}\right)\right) \quad\left(d_{1} \geq 2\right)$.

(2) $\quad\left(S L\left(d_{1}\right) \times S O(3) \times G L(2), \Lambda_{1} \otimes \Lambda_{1} \otimes \Lambda_{1}, V\left(d_{1}\right) \otimes V(3) \otimes V(2)\right)$ $\left(d_{1} \geq 5\right)$.

(3) $\quad\left(S L\left(d_{1}\right) \times S L(3) \times G L(2), \Lambda_{1} \otimes \Lambda_{1} \otimes \Lambda_{1}, V\left(d_{1}\right) \otimes V(3) \otimes V(2)\right)$ $\left(d_{1} \geq 3\right)$.

Now consider the case when $G$ is semi-simple. Then $(G L(1) \times G$, $\square \otimes \rho, V(1) \otimes V(d))$ is a non-regular P.V. with finitely many orbits and hence by Propositions 3, 6, and 7, it is equivalent to one of the following P.V.'s.

(1) $\left(S L(n), \Lambda_{1}, V(n)\right) \quad(n \geq 2)$.

(2) $\quad\left(S L(2 m+1), \Lambda_{2}, V(m(2 m+1))\right) \quad(m \geq 2)$.

(3) $\quad\left(S p(n), \Lambda_{1}, V(2 n)\right) \quad(n \geq 2)$.

(4) (Spin (10), half-spin rep., $V(16))$.

(5) $\quad\left(S L(n) \times S L(m), \Lambda_{1} \otimes \Lambda_{1}, V(n) \otimes V(m)\right) \quad(n<m)$.

(6) $\quad\left(S O(n) \times G L(m), \Lambda_{1} \otimes \Lambda_{1}, V(n) \otimes V(m)\right) \quad(n<m)$.

( 7$) \quad\left(S p(n) \times S L(2 m), \Lambda_{1} \otimes \Lambda_{1}, V(2 n) \otimes V(2 m)\right) \quad(n<m)$.

(8) $\left(S p(n) \times S L(2 m+1), \Lambda_{1} \otimes \Lambda_{1}, V(2 n) \otimes V(2 m+1)\right)$.

(9) $\left(S L(2 m+1) \times S L(2), \Lambda_{2} \otimes \Lambda_{1}, V(m(2 m+1)) \otimes V(2)\right)$.

(10) $\quad\left(S L\left(d_{1}\right) \times S O(3) \times S L(2), \Lambda_{1} \otimes \Lambda_{1} \otimes \Lambda_{1}, V\left(d_{1}\right) \otimes V(3) \otimes V(2)\right)$ $\left(d_{1} \geq 7\right)$. 
(11) $\quad\left(S L\left(d_{1}\right) \times S L(3) \times S L(2), \Lambda_{1} \otimes \Lambda_{1} \otimes \Lambda_{1}, V\left(d_{1}\right) \otimes V(3) \otimes V(2)\right)$ $\left(d_{1} \geq 7\right.$ or $\left.d_{1}=5\right)$.

Therefore we obtain the following theorem.

THEOREM 8. (i) A trivial P.V. with finitely many orbits is equivalent to one of the following P.V.'s.

(1) $\quad\left(S L(n) \times G L(m), \Lambda_{1} \otimes \Lambda_{1}, V(n) \otimes V(m)\right) \quad(1 \leq n \leq m)$.

(2) $\quad\left(S O(n) \times G L(m), \Lambda_{1} \otimes \Lambda_{1}, V(n) \otimes V(m)\right) \quad(3 \leq n \leq m)$.

(3) $\quad\left(S p(n) \times G L(m), \Lambda_{1} \otimes \Lambda_{1}, V(2 n) \otimes V(m)\right) \quad(2 n \leq m)$.

(4) $\quad\left(S O(3) \times S L(2) \times G L(d), \Lambda_{1} \otimes \Lambda_{1} \otimes \Lambda_{1}, V(3) \otimes V(2) \otimes V(d)\right)$ $(d \geq 6)$.

( 5 ) $\quad\left(S L(3) \times S L(2) \times G L(d), \Lambda_{1} \otimes \Lambda_{1} \otimes \Lambda_{1}, V(3) \otimes V(2) \otimes V(d)\right)$ $(d \geq 6)$.

(1') $\quad\left(S L(n) \times S L(m), \Lambda_{1} \otimes \Lambda_{1}, V(n) \otimes V(m)\right) \quad(1 \leq n<m)$.

$\left(2^{\prime}\right) \quad\left(S O(n) \times S L(m), \Lambda_{1} \otimes \Lambda_{1}, V(n) \otimes V(m)\right) \quad(3 \leq n<m)$.

$\left(3^{\prime}\right) \quad\left(S p(n) \times S L(m), \Lambda_{1} \otimes \Lambda_{1}, V(2 n) \otimes V(m)\right) \quad(2 n<m)$.

$\left(4^{\prime}\right) \quad\left(S O(3) \times S L(2) \times S L(d), \Lambda_{1} \otimes \Lambda_{1} \otimes \Lambda_{1}, V(3) \otimes V(2) \otimes V(d)\right)$ $(d \geq 7)$.

$\left(5^{\prime}\right) \quad\left(S L(3) \times S L(2) \times S L(d), \Lambda_{1} \otimes \Lambda_{1} \otimes \Lambda_{1}, V(3) \otimes V(2) \otimes V(d)\right)$ $(d \geq 7)$.

(ii) A non-trivial non-reduced $P . V$. with finitely many orbits is equivalent to one of the following P.V.'s.

(1) $\quad\left(S O(n) \times G L(m), \Lambda_{1} \otimes \Lambda_{1}, V(n) \otimes V(m)\right) \quad(n>m>n / 2)$.

(2) $\quad\left(S p(n) \times G L(m), \Lambda_{1} \otimes \Lambda_{1}, V(2 n) \otimes V(m)\right) \quad(2 n>m>n)$.

(3) $\quad\left(S O(3) \times S L(2) \times G L(5), \Lambda_{1} \otimes \Lambda_{1} \otimes \Lambda_{1}, V(3) \otimes V(2) \otimes V(5)\right)$.

(4) $\quad\left(S L(4) \times S L(3) \times G L(2), \Lambda_{1} \otimes \Lambda_{1} \otimes \Lambda_{1}, V(4) \otimes V(3) \otimes V(2)\right)$.

(5) $\quad\left(S L(5) \times S L(3) \times G L(2), \Lambda_{1} \otimes \Lambda_{1} \otimes \Lambda_{1}, V(5) \otimes V(3) \otimes V(2)\right)$.

$\left(1^{\prime}\right) \quad\left(S p(n) \times S L(2 m+1), \Lambda_{1} \otimes \Lambda_{1}, V(2 n) \otimes V(2 m+1)\right)$

$(2 n>2 m+1>n)$.

$\left(2^{\prime}\right) \quad\left(S L(5) \times S L(3) \times S L(2), \Lambda_{1} \otimes \Lambda_{1} \otimes \Lambda_{1}, V(5) \otimes V(3) \otimes V(2)\right)$.

Remark 9. Theorem 8 says that a P.V. with finitely many orbits is reduced with few exceptions. However, the orbital decomposition is necessary to prove that such a P.V. has actually finitely many orbits. The orbital decomposition of reduced P.V.'s is in almost all cases completed by the second author (see [12]), J. Igusa (see [2]), I. Ozeki (see [20]), Kawahara (see [13]) and S. J. Harris (see [7]). These orbital structures have very close relations with the $b$-functions and Fourier trans- 
forms of the relative invariants.

\section{REFERENCES}

[1] H. Weyl, Classical Groups, Princeton University Press, 1964.

[2] J. Igusa, A classification of spinors up to dimension twelve, Amer. J. of Math., $92(1970)$

[ 3 ] C. Chevalley, The Algebraic Theory of Spinors, Columbia University Press, 1954.

[4] C. Chevalley and R. D. Schafer, The exceptional simple Lie algebras $F_{4}$ and $E_{6}$, Proc. Nat. Acad. Sci. U.S.A., 36 (1950).

[5] N. Jacobson, Exceptional Lie Algebras, lecture note, Dekker, 1971.

[6] R. C. King, The dimensions of irreducible tensor representations of the orthogonal and symplectic groups, Can. J. Math., XXIII, 1 (1971), 176-188.

[7] Stephen J. Harris, Some irreducible representation of exceptional algebraic groups, Amer. J. of Math., 93 (1971).

[8] Y. Matsushima, Espace homogènes de Stein des groupes de Lie complexes, Nagoya Math. J., 16 (1960), 205-218.

[9] Y. Matsushima, Theory of Lie algebras (in Japanese), Kyoritsu Gendai Sugaku Koza, 15 (1956).

[10] N. Iwahori, Theory of Lie groups (in Japanese), Iwanami Koza Gendai Oyo Sugaku.

[11] M. Sato, Theory of prehomogeneous vector spaces (note by T. Shintani in Japanese), Sugaku no ayumi, 15 (1970), 85-157.

[12] T. Kimura, Study of irreducible prehomogeneous vector spaces, Master Thesis in Japanese, University of Tokyo, 1973, $373 \mathrm{pp}$.

[13] H. Kawahara, Prehomogeneous vector spaces related with the spin group, Master Thesis in Japanese, University of Tokyo, 1974.

[14] T. Suzuki, Fourier transforms of relative invariants of prehomogeneous vector spaces, Master Thesis in Japanese, Nagoya University, 1975.

[15] H. Sato, Functional equations of zeta-functions in several variables associated with some prehomogeneous vector spaces of positive definite hermitian matrices, Master Thesis in Japanese, University of Tokyo, 1975.

[16] M. Muro, Micro-local calculus and Fourier transforms (in prehomogeneous vector spaces), Master Thesis in Japanese, Kyoto University, 1976.

[17] T. Shintani, On Dirichlet series whose coefficients are class numbers of integral binary cubic forms, J. Math. Soc. Japan, 24 (1972).

[18] M. Sato and T. Shintani, On zeta functions associated with prehomogeneous vector spaces, Ann. of Math., 100, 1 (1974), 131-170.

[19] T. Shintani, On zeta functions associated with the vector space of quadratic forms, J. Fac. Sci. Univ. Tokyo, 22 (1975), 25-65.

[20] I. Ozeki, Micro-local structure of some prehomogeneous vector spaces, in preparation.

[21] D. Luna, Sur les orbites fermées des groupes algébriques réductifs, Inventiones Math., 16 (1972), 1-5.

The Research Institute for Mathematical Science

Kyoto University

Nagoya University and

The Institute for Advanced Study

Princeton 